\title{
An Empirical Study Investigating the Predictors of Software Metric Correlation in Application Code and Test Code.
}

\section{by}

Daniel Kwame Dapaah Afriyie

A thesis submitted to the Faculty of Graduate and Postdoctoral Affairs in partial fulfillment of the requirements for the degree of

Master of Applied Science

in

Electrical and Computer Engineering

Carleton University

Ottawa, Ontario

(C) 2019, Daniel Kwame Dapaah Afriyie 


\section{Abstract}

On non-trivial software, the large test code base needs adequate maintenance similarly to the application code. It has often been argued that test code should be simple, some authors even arguing that a test script should be reduced to a single control flow. If this is indeed what happens in practice, then we believe test code maintenance will be very different from application code maintenance, despite the fact that both are source code. Using a number of large open source software, we compare application code and test code by using a series of well-known source code complexity metrics. The results reveal that application code is, as expected, more complex than test code but not necessarily so much more complex. The study also confirms the assertion that test code is not as simple as it should be, at least as advocated in textbooks, and may therefore be very complex to maintain.

Using complexity metrics to determine the difference, our findings also reveal that, the kind of code determines the extent of monotonicity between the number of lines of code (LOC) and cyclomatic complexity (CC). While a number of authors hypothesize and experimentally confirm that $\mathrm{CC}$ has a very strong correlation with LOC, justifying the use of LOC in place of CC (and Halstead Effort), we believe that this strong correlation is prevalent only in production code as results from test code prove otherwise. In test code, there exist a very weak (or almost no) correlation between (a) LOC and CC, (b) Halstead Effort and CC, and (c) LOC and Halstead Effort. We therefore argue that the level of correlation depends on at least three factors namely: the kind of code, the kind of software and the kind of metric. Therefore, we believe it would be inappropriate, without considering these factors to substitute one metric with another using some generalized correlation hypothesis. The results gained thus far contradict the notion that some metrics are correlated (that is if the right factors are not considered).

Given the weak monotonicity between CC and LOC, CC and Halstead Effort for test code, we disagree that $\mathrm{CC}$ and Halstead metrics are redundant as some studies suggest. Rather, we advocate for the use of CC over LOC (or both or cylomatic density) due to numerous advantages $\mathrm{CC}$ has over $\mathrm{LOC}$ such as the fact that $\mathrm{CC}$ is perceived to better reflect cognitive complexity, numerical complexity, adequacy of testing, interdependency and code refactoring that cannot be accounted for simply by LOC. 


\section{Acknowledgements}

One great discovery of climbing mountains and winning battles is the realization of even greater mountains to climb and tougher battles to win. In doing so, we are faced with hard choices; one of which is flinching back, to the very least. The true test of our resilience is in our ability to ultimately win and endure climbing higher mountains. Without which many have lived their lives not having ever lived.

I am therefore grateful to Almighty God that this project is finally completed. A few years ago, a bleary-eyed stare at what lies in the future was unassuring, but the joining of the dots as time went on gives us this delight to be forever grateful and an assurance to be hopeful.

I owe special thanks most of all, to my supervisor, Prof. Yvan Labiche. Such a great inspiration. Everything he does, from what is believed to be conceptually complex to the least significant, are tackled with a great deal of perfection and a high level of academic proficiency. A mentor, a father, and a friend. You only get better by heeding to his advice. Very helpful, insightful and full of brilliance. I am privileged to work with him.

It was a great pleasure to collaborate with Prof. Natalia Stepanova at the School of Mathematics and Statistics for sharing ideas on complicated topics when organizing my thoughts on statistical principles and methodologies.

I feel so favored to enjoy special treatment from Jennifer Poll, Jenna McConnell and Darlene Hebert. Many thanks to them and members of the Department and Faculty. I am indebted to the many individuals who generously and without hesitation, supported in various ways.

The last stages of my masters had turmoil moments, to say the least. Thankfully, I had my fair share of great and reliable people who stood with me. A mentor Mr. George Cole, my sweetheart and good friend Abigail Oduro and a brother Eric Obeng.

I am grateful for the support and encouragement from my family, Samuel Afriyie, Mercy Afriyie and Isaac Afriyie. Where water fails, blood sticks better. I love you. 


\section{Table of Contents}

Chapter 1 INTRODUCTION ................................................................................................ 1

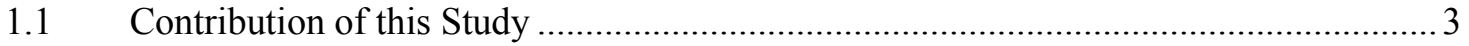

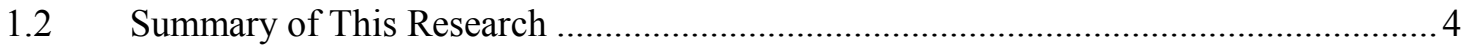

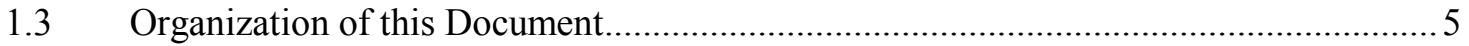

Chapter 2 RELATED WORK.............................................................................................. 6

Chapter 3 BACKGROUND INFORMATION ................................................................. 11

3.1 Cyclomatic Complexity, Lines of Code and Halstead Metrics. ................................... 11

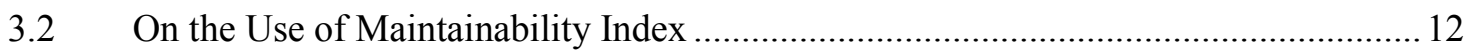

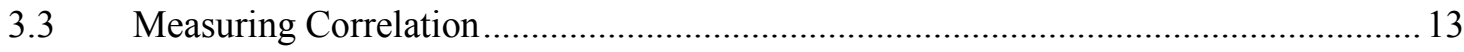

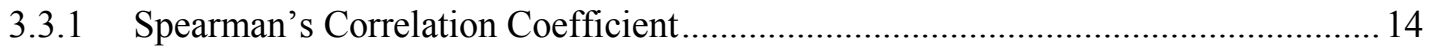

3.3.2 On Pearson's Correlation Coefficient .......................................................................... 15

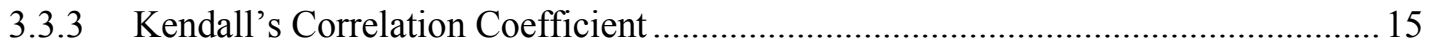

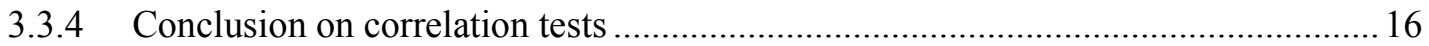

3.4 Test for Normality: Anderson Darling Test, Shapiro-Wilk, D’Agostino K-squared,

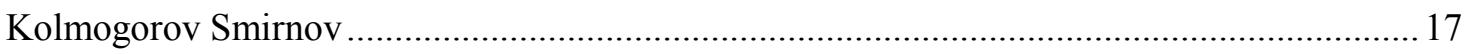

3.5 Homogeneity of Variance: T-Test and ANOVA.................................................... 18

3.6 Statistical Analysis - Wilcoxon Rank Sum Test ...................................................... 19

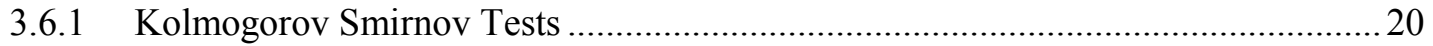

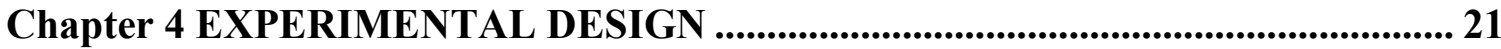

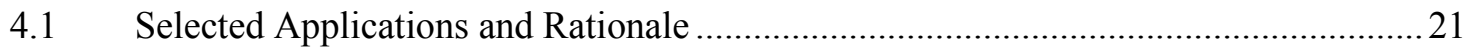

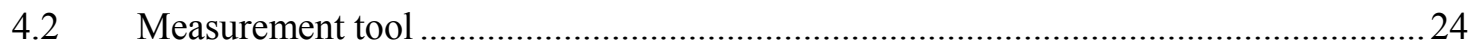

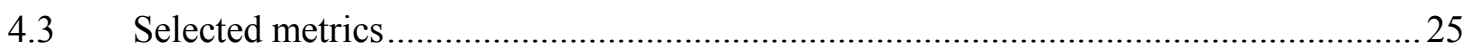

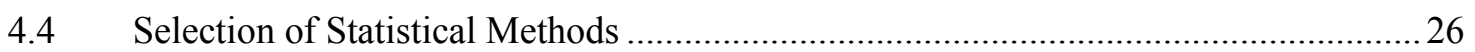

Chapter 5 RESULTS (ANALYSIS OF DATA) ................................................ 28

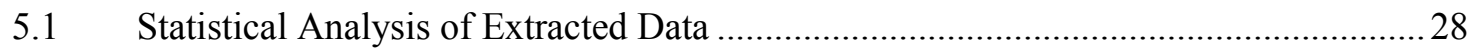

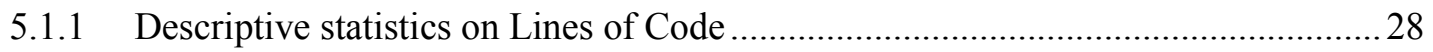

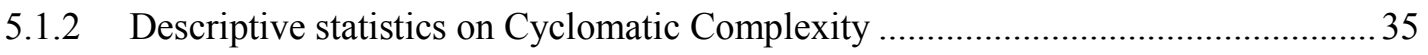

5.1.3 Descriptive statistics on Using Distributions, Graphs and Average Values for

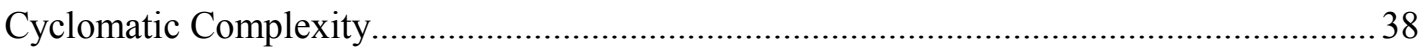

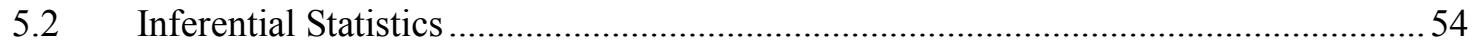

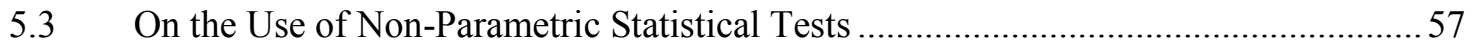


Chapter 6 THREATS TO VALIDITY ........................................................................... 65

Chapter 7 CONCLUSIONS ................................................................................................... 68

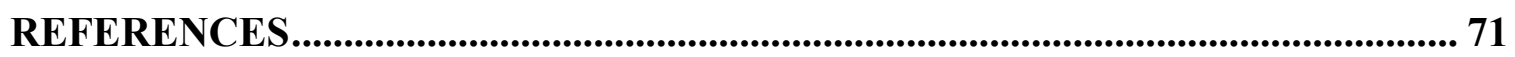

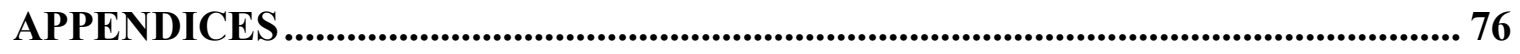

Appendix A Distributions of cyclomatic complexity values for objects................................. 76

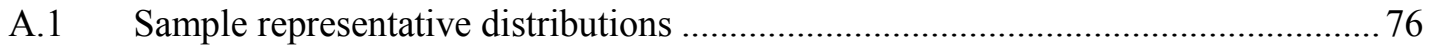

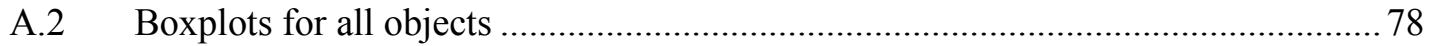

Appendix B : Linear Scale Zoomed In On The Left Quadrant ............................................... 80

B.1 Linear Scale Linear Scale Zoomed In On The Left Quadrant (Production Code) ... 80

B.2 Linear Scale Zoomed In On The Left Quadrant (Production Code) .......................... 85

Appendix C : Exporting Metric Values Using Scitols Version 4.0 …....................................90

C.1 Analyze Files or Directories Using SciTools Understand...................................... 90

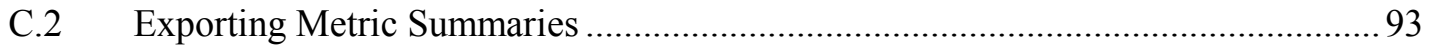

C.3 Collecting General Metric Values about Code …....................................................93

C.4 Collecting more detailed Metric values for Different Programming Languages ..... 94

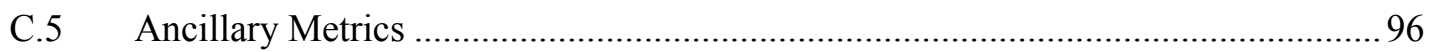

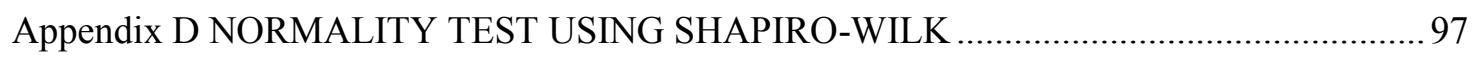

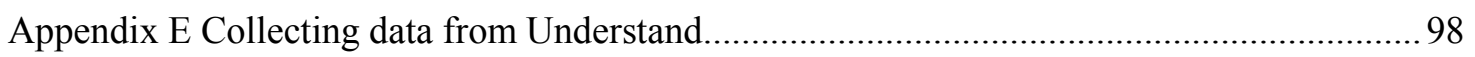

E.1 Export Metrics Using SciTools Understand Version 4.0 ....................................... 98

E.2 Basic Metrics with Methods Calls and Halstead using A Script............................... 98 


\section{List of Tables}

table 1. References Of Case Study Software Being Studied ….................................. 23

Table 2. Simple Metric Values For Software Objects .................................................. 32

Table 3. Metric Values (Cyclomatic And Halstead) For Software Objects .................... 41

Table 4. Maximum Cyclomatic Complexity And Median Values, Results For Wilcoxon's

Rank Sum Test, And Kolmogorov Smirnov Test............................................................ 53

Table 5. Correlation Coefficient, P-Values And Statistic For Comparing Cyclomatic

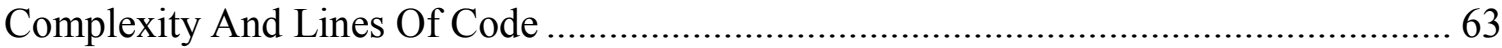

Table 6. Correlation Of Loc Vs Cc And Cc Vs Halstead Effort For Test Code............... 64

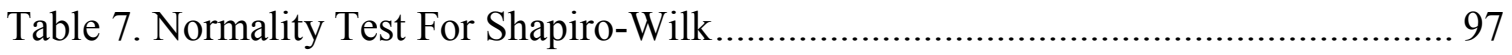




\section{List of Figures}

Figure 1. Distribution of cyclomatic complexity values for Apache Httpd AC .............. 39

Figure 2. Distribution of cyclomatic complexity values for Apache Httpd AC .............. 76

Figure 3. Distribution of Cyclomatic complexity values for Apache Httpd TC (logarithmic

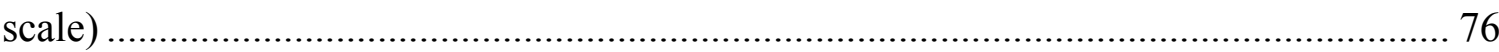

Figure 4. Distribution of cyclomatic complexity values for JFreechart AC

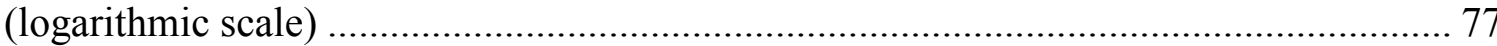

Figure 5. Distribution of cyclomatic complexity values for JFreechart TC

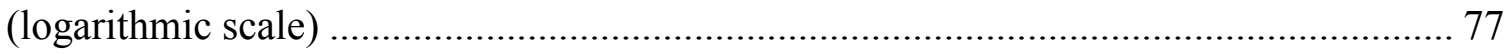

Figure 6. Box Plot of $\mathrm{CC}$ for objects with method $\mathrm{CC}$ values in range $[1,100] \ldots \ldots \ldots \ldots . . . .78$

Figure 7. Box Plot of $\mathrm{CC}$ for objects with method $\mathrm{CC}$ values in range $[1,500] \ldots \ldots \ldots \ldots . . . .78$

Figure 8. Box Plot of CC for objects with method CC values in range [1, 1000].......... 79

Figure 9. Box Plot of $\mathrm{CC}$ for objects with method $\mathrm{CC}$ values in range $[1,10000] \ldots \ldots . . .79$ Figure 10. Scatter Plot of LOC (x) vs CC (y) for Amorok, zoomed into the bottom left

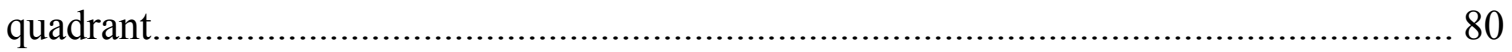

Figure 11. Scatter Plot of LOC (x) vs CC (y) for Curl, zoomed into the bottom left quadrant.

Figure 12. Scatter Plot of LOC (x) vs CC (y) for Ditaa, zoomed into the bottom left quadrant.

Figure 13. Scatter Plot of LOC (x) vs CC (y) for Handbrake, zoomed into the bottom left quadrant

Figure 14. Scatter Plot of LOC (x) vs CC (y) for Hibernate, zoomed into the bottom left quadrant 82

Figure 15. Scatter Plot of LOC (x) vs CC (y) for Hibernate ORM, zoomed into the bottom left quadrant. 82

Figure 16. Scatter Plot of LOC (x) vs CC (y) for Notepad++, zoomed into the bottom left quadrant.

Figure 17. Scatter Plot of LOC (x) vs CC (y) for Onion, zoomed into the bottom left quadrant 83 
Figure 18. Scatter Plot of LOC (x) vs CC (y) for VLC, zoomed into the bottom left quadrant 84

Figure 19. Scatter Plot of LOC (x) vs CC (y) for Open XML (PC), zoomed into the bottom left quadrant. 84

Figure 20. Scatter Plot of LOC (x) vs CC (y) for Amorok (TC), zoomed into the bottom left quadrant. 85

Figure 21. Scatter Plot of LOC (x) vs CC (y) for Curl (TC), zoomed into the bottom left quadrant 85

Figure 22. Scatter Plot of LOC (x) vs CC (y) for Curl (TC), zoomed into the bottom left quadrant 86

Figure 23. Scatter Plot of LOC (x) vs CC (y) for Handbrake (TC), zoomed into the bottom left quadrant. 86

Figure 24. Scatter Plot of LOC (x) vs CC (y) for Hibernate ORM (TC), zoomed into the bottom left quadrant. 87

Figure 25. Scatter Plot of LOC (x) vs CC (y) for Hibernate (TC), zoomed into the bottom left quadrant.

Figure 26. Scatter Plot of LOC (x) vs CC (y) for Notepad ++ (TC), zoomed into the bottom left quadrant. 88

Figure 27. Scatter Plot of LOC (x) vs CC (y) for Onion (TC), zoomed into the bottom left quadrant. 88

Figure 28. Scatter Plot of LOC (x) vs CC (y) for Open XML (TC), zoomed into the bottom left quadrant.

Figure 29. Scatter Plot of LOC (x) vs CC (y) for VLC, zoomed into the bottom left quadrant. 89

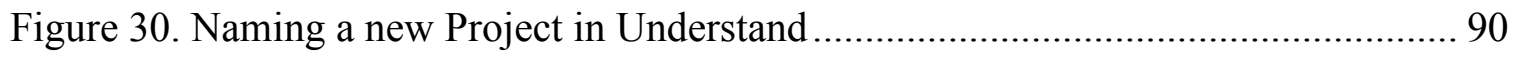

Figure 31. Selecting Source Code Languages Understand should Export ..................... 91

Figure 32. Choosing How to Import Files and Settings............................................. 91

Figure 33. Add Project Directory for Analysis in Understand ................................... 92

Figure 34. Complete the Project Configuration and Settings for Analysis..................... 92

Figure 35. Analysing and Extracting Source Code Information in Understand.............. 93

Figure 36. Exporting Metrics Summary for Source Code ............................................ 94 
Figure 37. Browse Detailed Metric Summary for Source Code ................................... 95

Figure 38. Extracting Detailed Metrics for Specific Programming Languages............... 95

Figure 39. Extract Ancillary Metrics from Understand............................................. 96

Figure 40. Specifying file paths to Run a Script Using Command in Understand ........... 99 


\section{Chapter 1 INTRODUCTION}

In the past years, there has been reports and intense research activities on the relevance of test code and its positive impact on code quality in the software development. Schemes, hypothesis, and many algorithms have been developed to well maintain software. Verification of software by means of testing is the most common situation. On non-trivial software, software testing leads to a lot of test code. Depending on the context, anecdotal evidence indicates that one often needs to create one line of code (LOC) of test code for every LOC of application code and that this ratio can sometimes reach 1.5 or even in specific (e.g., safety critical applications) cases, four: in summary, one to four LOC of test code for every LOC of application code being tested. This large test code base needs maintenance, similarly to application code.

To adequately maintain test code, we need to understand it. Some authors argue that test code should be as simple as possible, sometimes limited to one straight sequence of statements (no control flow structure) [41], though this is not necessarily the case in practice. Additionally, test code can be expected to follow specific templates, the most common one being that a test case should include a set-up, some execution of the piece of software under test, some collecting of data for the oracle, an oracle assertion and a tear down. Last, some test cases are written with open source frameworks, such as xUnit, JUnit and the like, given their code a rather specific structure.

Maintaining application code often involves source code analysis techniques such as code smells or clone detection, which can lead to some refactoring of the code when this is warranted. Given the hypothesized differences mentioned above between application code and test code, we argue test code maintenance may look different to application code maintenance. For instance, assuming test code is indeed less complex than application code to the extent that a test code method is nearly a linear sequence of un-interrupted statements (no control flow), then a maintenance procedure that would prioritize refactoring based on high cyclomatic complexity values or the presence of for-loop statements (which have been shown to be less understandable than if statements [43]) would not be able to discriminate test methods and would be useless (i.e., the distribution of complexity values to prioritize from is flat). If, when maintaining test code, one wants to extend empirical results obtained by studying application code, for instance, to deploy some application code maintenance 
techniques which have proved to be successful on application code, one must first evaluate whether test code is sufficiently similar to application code. (Note that this notion of similarity will need to be clarified and will necessarily be specific to the maintenance technique being considered. This kind of study is out of the scope of this work.)

This study is a first step towards identifying to what extent test code differs from application code. We stress here that this is a first step because we decided to focus on open source applications we collected online, and that we consider only one aspect of complexity as measured by standard metrics. Also, we do not distinguish between the different kinds of tests (e.g., unit/integration/system testing, functional/non-functional testing), which may also likely affect the linearity of the correlation of complexity metrics; for instance one may expect a unit test to likely be a linear sequence of statements whereas a non-functional (e.g., stress) test may require a complex set-up with complex control flow.

The results we discuss in this paper may therefore not (entirely) apply to industry software. We indeed expect industry code to lead to different results for two reasons (among others): the ratio test code LOC over application code LOC is likely higher in industry than in open source (e.g. we believe a 4:1 ratio is unheard of in open source whereas this is relatively common in industry, at least with the private organizations we engage with); some industry developed their own version of a test execution infrastructure that does not necessarily resemble xUnit, JUnit and the like, whereas these framework, that give a specific structure to test code, are pervasive in open source.

To identify whether and to what extent test code differs from application code, we turned to source code complexity metrics which have been shown to relate to maintenance tasks, source code understanding ... [3]. In this study, we therefore ask: How do application code and test code differ according to standard code complexity measures? How do these complexity software measures relate? If they do, to what extent are they related?

In the past years, there has been reports and intense research activities on software complexity, code maintenance and metric correlation using well-known metrics such as Cyclomatic complexity, Halstead metrics and Lines of Code, to predict code quality, bug proneness, and code maintenance, and this took place largely on production Code [66]. On the use of metrics such as Cyclomatic complexity, Lines of Code and Halstead Effort, numerous studies have been conducted to predict the extent to which some metrics are 
correlated, and that, should they do, it would suffice to use one in place of the other(s). While these experimental results confirm some correlation, as confirmed by our results, this is somewhat specific to production code and not necessarily test code. Not much research has studied whether these metrics correlate also for test code. In our study, our investigation reveals that this hypothesis only holds when considering production code as it is the exact opposite in test code. We hypothesize that, the correlation that exist between some metrics does not merely depend on the type of metrics (size, complexity, cohesion, and coupling), rather, it depends more on the kind of software (open source application or industry application), and the kind of code (production code or test code).

One critical challenge that numerous studies and hypothesis [47], [48], [49], [50], [51] have not explored on correlation of metrics is the factors that affect the correlation being: the kind of code, the kind of software and the kind of metric. This is evident in the large-scale software study conducted by Landman and colleagues [49], the work of Maine and Miguel [47] and many others. The 1.2 million source files of $\mathrm{C} / \mathrm{C}++$ and Java production code analysed by Graylin and colleagues [51] present a verifiable evidence of a stable relationship between Lines of Code (LOC) and cyclomatic complexity (LOC) but lacks an analysis for test code and a quintessential lucid justification for advocating for the use of LOC over CC. Landman's work is limited to only Java methods, which is not a reflection of all programming languages. Also on Landman's work, the idea of data transformation is very arguable and does not reflect the authenticity and originality of the data collected. While we do agree that it really makes sense to transform non-normal data to a normal one, we are skeptical (disagree) with the results it produces as this can be very misleading [51], [52]. In addition, on the issue of normality, Landman presents a series of results gained from a transformed non-normal distribution, using parametric tests on the transformed data, which though this is a procedure accepted in the field, seems inappropriate and misleading. In our work, we investigate their claim by extending the work to 667,868 methods of 40 open source software applications and five Industry applications written in $\mathrm{C}, \mathrm{C}++, \mathrm{C \#}$, Python and Java.

\subsection{Contribution of this Study}

This research provides a unique contribution to test code maintenance by advancing the understanding of how Application Code and Test Code Differ. Previous literature 
hypothesises a low complexity for test code and suggests test code should be as simple as possible, sometimes limited to one straight sequence of statements (no control flow structure). In contrast, our research shows this is not necessarily the case in practice. This research provides a fresh insight and clarity on the correlation of software complexity metrics. This study advances the theory of correlation by demonstrating that the strong correlation between complexity metrics (LOC, CC and Halstead metrics) is not a characteristic of the metrics but rather appears to depend on the kind of source code one measures. This research introduces the idea that the perceived strong correlation is only prevalent in application code and not test code and industry code. The degree of correlation relies on three factors namely: the kind of code (application vs. test code), the kind of software (obtained from an industry partner or online/open source) and the kind of metrics (LOC, CC, Halstead).

\subsection{Summary of This Research}

This thesis contributes to software maintainability and ways of analyzing application code and test code. In this study, we learn that:

- When measuring the extent of software complexity, Application Code is more complex than Test Code;

- Test Code is not as simple as it is supposed to be and can be extremely complex;

- The correlation between software metrics depends on factors such as the kind of software (open-source vs. industry), the kind of code (application vs. test code) and the kind of metric (LOC, CC, Halstead);

a) We confirm that there is a correlation between LOC, CC and Halstead Effort, however, this is only prevalent in application code

b) We identify that there is no such correlation for test code and therefore, the notion of correlation of LOC, CC and Halstead effort does not apply here.

c) The correlation for test code drops even further as we explore industry applications.

- Attempting to substitute one source code complexity metric with another should take into account the factors that affect the level of correlation between these metrics. 


\subsection{Organization of this Document}

The remainder of the thesis is structured as follows. In Chapter 2, we investigate the depth of related work. We provide background on source code metrics and statistical analysis in Chapter 3. Chapter 4 describes the experiment we conducted. 0 discusses results. We discuss threats to validity in Chapter 6 and conclude in Chapter 7. 


\section{Chapter 2 RELATED WORK}

A large number of authors have published on issues relating to software metrics largely due to their relevance when evaluating the quality of a piece of code. While we find most of them useful, not many have addressed salient issues regarding the extent of complexity that exist between different software artefacts. A select few of the several closely related studies are considered in this research work to gain a good understanding of the different facets of software quality, metric correlation and code complexity.

Related work in our context pertains to three different topics: (a) complexity measurement of code, including test code, (b) the correlation of complexity metrics and (c) the relative complexity of test code and the application code it tests.

We have not found any piece of work in our literature search on the Scopus database that discusses whether test code tends to have a different complexity than the application code it tests. There exist works on different notions of test quality, but these only look at test code, independently of the application code it tests. For instance, authors have discussed test patterns, that is, good design principles to code tests [39]. Others have tried to estimate test quality by considering the presence of assertions in code and the structural coverage of the application code achieved by the tests, among other things, thereby moving beyond the mere measurement of structural coverage of application code achieved by tests [40].

This chapter is not meant to be a systematic literature review or systematic mapping study about source code metrics, their definitions, their use... Instead we intend to report on a number of salient pieces of work which will shed some light on what we are doing and why.

Regarding code complexity, a plethora of source code metrics have been defined over the years [3]. Some are very simple, such as counting the number of lines of code (LOC), i.e. not counting comments and blank lines, or the number of lines of comments. Some are more complex to compute though there is tool support, such as the cyclomatic complexity. We can evaluate source code by collecting metric values for McCabe's cyclomatic complexity, Halstead's Effort, Difficulty, and Maintainability index, and other metrics such as the number of methods, the number of classes (in an object-oriented software) or the number of lines of code [33]. 
Through a systematic mapping study of 226 studies published from 2010 to 2015, Nuñez-Varela and colleagues show that some of the most used software metrics that are used to study various software engineering activities include Lines of Code (LOC) and cyclomatic complexity (CC), when one omits the metrics that are not common to different paradigms (e.g., after removing object-oriented metrics) [3].

Over the past decades, Cyclomatic Complexity (CC) has gained more attention in literature and has proven useful as a heuristic means of analyzing the cognitive complexity of a piece of code [3] rather than the traditional use of numerical complexity values reported by its counterpart, Lines of Code.

While some authors envision Cyclomatic Complexity as a tool to test the complexity of a source code, others focus on the use of traditional Lines of Code (LOC) as a better and much easier substitute to Cyclomatic Complexity due to the strong correlation that has been reported to exist between the two code quality metrics. We also note that there is an ongoing, unresolved debate about the usefulness of the Cyclomatic Complexity metric. While numerous research studies (e.g., [43], [44], [45], [48], [50]) have argued in favor of the existence of a strong correlation between Cyclomatic Complexity and Lines of Code, and therefore advocate for the use of only one of them, namely LOC because it is simpler to compute, some authors on the contrary claim that the correlation between CC and LOC is only moderate when the source code is analyzed in modules rather than as an agregate unit (larger program) [49], [51] as claimed by the former. Though our intent is not to settle the ongoing debate, we, however, investigate these claims for application code and test code at the method level (lowest level of granularity for complexity metrics) and as an aggregate unit using large open source applications written in multiple programming languages to verify the validity of the claim for those two different kinds of code. We investigate the possible reasons for these claims, what is missing in the experiment leading to these varying hypotheses and what contributes to the differences in their outcomes.

Through the analysis of 1.2 million files written in $\mathrm{C}, \mathrm{C}++$ and Java, Jay and colleagues discover a strong linear relationship between Cyclomatic Complexity and LOC [51], to the extent that the authors argue the two metrics may likely be measuring the same source code complexity characteristics. 
Meine and Miguel [47] investigate $\mathrm{C}$ and $\mathrm{C}++$ programs under four common metrics (Lines of Code, Lines of comment, Halstead Effort, and Cyclomatic Complexity) using distribution graphs and density plots of LOC/Halstead Volume and LOC/CC. They find Lines of Code and Halstead Volume to be strongly correlated, and a higher (an even stronger) correlation between Cyclomatic Complexity and Lines of Code.

Similarly, Meulen and Revilla find that the Cyclomatic Complexity and Halstead volume metrics are correlated to LOC but not correlated to software dependability metrics [23].

Graylin and colleagues [51] strongly disagree with the reasons some practitioners prefer to use CC in place of LOC. Rather, they advocate substituting CC with LOC due to the perfect and stable linear relationship between $\mathrm{CC}$ and LOC across all platforms $(\mathrm{C}, \mathrm{C}++$, Java) they observe on 1.2 million source files randomly selected on SourceForge. With five select projects (from NASA), they justify the necessity of statistical analyses like distribution of variance and Persons Correlation coefficient in measuring the distinction between the two metrics. The work though presents a strong correlation between $\mathrm{CC}$ and LOC, however it claims CC but does not lay any strong justifiable reason why LOC should be preferred over $\mathrm{CC}$ as they believe $\mathrm{CC}$ and LOC measure the same property - which to some extent, is very arguable as $\mathrm{CC}$ rather presents a more convincing complexity measure that is helpful in cognitive complexity, numerical complexity, adequacy of testing, interdependency and code refactoring that cannot be accounted for simply by LOC. . With no clear justifiable reasons given, one could as well argue in favor of $\mathrm{CC}$ with the same set of data and analyses made, justifying why $\mathrm{CC}$ should be used instead, for a variable number of reasons, due to the strong correlation. The presence of a correlation between certain kinds of seemingly related software metrics does not always guarantee a substitution as they measure different properties. For example, Lines of Code may help in determining the number of test cases for $100 \%$ statement coverage and not branch coverage. Cyclomatic complexity on the other hand due to the number of linearly independent paths it measures, predicts the number of test cases need for $100 \%$ decision coverage which implies $100 \%$ branch coverage and $100 \%$ statement coverage. The same is for the cognitive complexity where the conceptual difference in understanding two different 100-line code with different $\mathrm{CC}$ makes $\mathrm{CC}$ a better substitute. These and many reasons, just to name a few. 
Adnan and Enoiu [50] address the issue of complexity metrics and software testing and in their study, investigate whether the number of test cases in a test suite and the amount of time it takes to execute (test effort) are correlated with the software metrics they selected, on safety-critical industry control software. They report a moderate correlation between test effort and software complexity metrics (LOC, CC, Halstead complexity (HC) and Information Flow Complexity (IFC)). Also the size of a software highly affects the amount of test cases and execution time and therefore, directly affects the test effort required to maintain the software.

All these studies [43], [44], [45], [48], [50] report similar finding to what has already been said earlier, that is Lines of Code (LOC), cyclomatic complexity (CC) and Halstead Effort (HE) are correlated.

Henry and colleagues [46] in their measurement of the correlation between three complexity metrics, specifically Cyclomatic complexity, Halstead Effort and Henry and Kafura's information flow, observe CC and Halstead effort are linearly related whereas Henry and Kafura's Information flow is regarded as an independent complexity metric. Using a single UNIX Operating System as the sole case study, the results are somehow challenged due to the limited number of case studies presented in the paper and the exclusive use of correlation coefficient.

In another study [44], Abdullah Al Mamun and colleagues establish the relationship (inter-relationship and intra-relationship) between 25 software metrics, classified into size, documentation, complexity and duplication related metrics, in an empirical study on 20 open source software with 9,572 revision, from eight "well-known" companies. Results indicate a high correlation among size related metrics. Complexity related metrics and Documentation related metrics on the other are not as strongly correlated among themselves (intra-relationship), within the same category as they are with size related metrics. Duplication related metrics are more correlated with themselves (intra-relationship) than with metrics of other domains (inter-relationship).

Abdullah Al Mamun and colleagues use 21 open source applications to determine whether the hypothesized four different types of measurement (cumulative, organic, density and average) used in software correlation have some sort of influence on the correlation of the metrics they selected for these types [45]. The collinearity and correlation of software 
metrics in a given project is strongly affected by the measurement approach being used. In their study, they identify the four categories of measurement that is "cumulatively": a measure of the running sum of various revisions or commit of a single project whereas "organic" to describe a measurement where a single revision is taken into account and, if necessary sometimes, compared with previous versions. "Density" is a description of the per unit ratio of a given metric; for instance:

$$
\begin{aligned}
& \text { Comment_line_density }=\frac{\text { number_of_comment_lines }}{\text { LOC+ number_of_comment_lines }} * 100 \\
& \text { Cyclomatic_Complexity_density }=\frac{\text { Cyclomatic Complexity }(C C)}{\text { Lines of Code }(L O C)}
\end{aligned}
$$

An "Average" gives the mean values of a given metric.

These results are somewhat refuted by the analysis of millions of Java and $\mathrm{C}$ functions conducted by Landman and colleagues [34]; the authors conclude that the relationship between Cyclomatic Complexity and LOC is not that strong and argue in favor of using the two metrics. 


\section{Chapter 3 BACKGROUND INFORMATION}

In this chapter, we delve into software complexity metrics and common procedures for measuring and analyzing scientific data including statistical methods, metric correlation coefficients, normality tests and analysis of variance (homoscedasticity). We also describe the relevance of normality assumptions and its significance when constructing reference intervals and the choice of statistical procedures (parametric and non-parametric tests).

\subsection{Cyclomatic Complexity, Lines of Code and Halstead Metrics.}

Cyclomatic complexity (CC) is a well-known complexity measure and has proven useful as a standard metrics [3] for analyzing the cognitive complexity of a piece of code. It is often mentioned with Lines of Code and Halstead Effort.

The Cyclomatic Complexity [1] takes the approach of measuring the number of linearly independent paths in the code to determine its complexity. Linearly independent paths in the code are due to control flow structures such as if statements and loops. The Cyclomatic Complexity, denoted V(G), of a piece of code represented by its control flow graph $\mathrm{G}$ with $\mathrm{n}$ vertices, e edges and $\mathrm{p}$ connected components is: $V(G)=e-n+p$. Code with a low Cyclomatic Complexity value has shown to be more stable than code with a high Cyclomatic complexity value [5]. Some authors provide guidance about the use of this metric [5]: A Cyclomatic Complexity value between 1 and 10 represents code that is simple, easy to test and modify with few risks due to the low number of paths and conditional statements; A Cyclomatic complexity value between 11 and 20 is for a piece of code that is understandable, maintainable and testable at a moderate level of risk; A Cyclomatic Complexity value between 21 and 50 represents code that is highly risky to maintain, test and modify; A cyclomatic complexity value above 50 means a practically unmaintainable piece of code.

Halstead instead envisions complexity [60] in terms of difficulty, effort needed to understand, algorithmic volume, by considering the number of distinct operands and operators. The program volume, $\mathrm{V}$, is a measure of the (algorithmic) size of the program, and is defined as follows: $V=N_{1}+N_{2} \log _{2}(n 1+n 2)$; where $\mathrm{n}_{l}$ is the number of distinct operators, $\mathrm{n}_{2}$ is the number of distinct operands, $\mathrm{N}_{l}$ is the total number of occurrences of operators, and $\mathrm{N}_{2}$ is the total number of occurrences of operands. The difficulty of handling 
a program, $\mathrm{D}$, is measured as: $D=\frac{n 1}{2} * \frac{N_{2}}{n 2}$. The Effort $(\mathrm{E})$ required to understand the logic and algorithmic implementation of a program is given as a product function of the difficulty and the volume: $E=D^{*} V$. Last, the Maintainability Index (MI) is calculated in terms of Halstead Volume, McCabe Cyclomatic Complexity and Lines of Code:

$$
\begin{gathered}
M I=171-5.2 * \operatorname{In}\left(V^{\prime}\right)-0.23 * V(G)^{\prime}-16.2 * \operatorname{In}\left(L O C^{\prime}\right) \\
+50 \sin \sqrt{2.46 * \text { RatioCommentToCode }}
\end{gathered}
$$

The choice on metrics is to construct a composite metric that factors the density of operators and operators (Halstead Volume), the logical complexity of code (cyclomatic complexity), the size of the code (Lines of Code) and the amount of comments [77].

\subsection{On the Use of Maintainability Index}

Maintainability index is a single-value index that measures the relative ease of maintaining a piece of code using a factored formula of Halstead Volume (V), Lines of Code (LOC) and cyclomatic complexity (CC) proposed by Oman and Hagameister [75]. In the estimation of Maintainability Index (MI) using regression models, Coleman-Oman [72] introduces the number-of-comments parameter in the Maintainability index equation and proposes a more satisfactory model (Model 2) [74] due to the sensitivity of the initial model of MI (Model 3, proposed by Oman [72], [75]) to large amounts of comments. This behavior has the tendency of increasing the Maintainability index especially for a small piece of code heavily doped with a large block of meaningful (relevant) comments. The introduction of a "threshold" value of 50 and the average comment lines per module (perCM) corrects this defect. In using the formula in Model 2, we observe a sinusoidal curve with an upper and lower limit of +-50. Welker and Oman [76] advocate using regression models 1 and 2 while ensuring that assumptions of using any [74] are satisfied (explained later). Aldo Liso [74] contributes to this by introducing a constant, $\mathrm{K}$ (in the Model 4 variant) that accounts for the peaks, decreasing paths, and the function path. Model 4 (a recent study) is a variation of Model 2 that introduces the multiplicative constant K for issues addressed in [74].

$$
\begin{aligned}
& M I_{1}=171-5.2 \operatorname{In}\left(V^{\prime}\right)-0.23 V(G)^{\prime}-16.2 \operatorname{In}\left(L O C^{\prime}\right) \\
& M I_{2}=M I_{1}+50 \sin \sqrt{2.46 p \operatorname{erCM}} \\
& M I_{3}=171-3.42 * \operatorname{In}\left(E^{\prime}\right)-0.23 * V(G)^{\prime}-16.2 * \operatorname{In}\left(L O C^{\prime}\right)+C M^{\prime} \\
& M I_{4}=M I_{1}+50 \sin \sqrt{K * 2.46 p \operatorname{erCM}}
\end{aligned}
$$


Where $\mathrm{V}^{\prime}$ is the average Halstead Volume

$\mathrm{V}(\mathrm{G})$ ' is the average extended cyclomatic complexity

LOC' is the average Lines of Code.

perCM is the average percent of Lines of Comment per Module.

E' is the average Halstead effort and

$\mathrm{CM}^{\prime}$ is the average number of comments per submodule or method.

$\mathrm{MI}_{2}$ is a variant of MI relevant for situations where comments are an essential part of the code especially for code that is heavily loaded with such comments. It is important and worth nothing the selection of MI more depends on the amount of comments contained in the code. For a piece of code with no comments or whose comments do not contribute to in any form to the code, $\mathrm{MI}_{1}$ suffices. Else, $\mathrm{MI}_{2}$ is recommended in all cases where comments are likely. Assumption of Model 2 are listed in [74][77]. Coleman and Oman [73] present thresholds for interpreting the results obtained for MI defined as:

$$
\begin{aligned}
& M I<65 \text { as poor maintainability (difficult to maintain), } \\
& 65 \leq M I<85 \text { as fair or moderate maintainability, and } \\
& 85 \leq M I \text { as excellent maintainability. }
\end{aligned}
$$

A high value of MI (above 85) suggests a code with better maintainability while that below 65 is believed to be difficult to maintain. At this point it is right appropriate to observe the maximum, minimum, average CC and Halstead Volume (V) and compare with the MI if, perhaps, there is some sort of coherence in the level of complexity (as the polynomial of MI uses $\mathrm{CC}$ and $\mathrm{V}$ ). The nature of the sinusoidal curve of the polynomial presented in the regression model 2 makes it is possible to have the median, mean and max MI values larger than the overall MI as the value depends on the variation of the values for the parameters (that is $\mathrm{V}, \mathrm{V}(\mathrm{G})$, and LOC) of the logarithmic function of MI.

\subsection{Measuring Correlation}

We first discuss separately three of the main correlation statistical tests and then conclude on their respective advantages and drawbacks. In this section, we explain some statistical concepts and provide useful information on Spearman's correlation coefficient in 3.3.1, Pearson's correlation coefficient in section 3.3.2, Kendall's correlation coefficient in section 3.3.3 and conclusions on correlation tests in section 3.3.4. 


\subsubsection{Spearman's Correlation Coefficient}

In a quantitative analysis, the Spearman's Correlation, a non-parametric method, is used to establish whether a monotonic relationship exists between non-normal bivariate datasets using their ranked values. It measures the strength and the direction of the relationship to derive a correlation between the two variables. It requires ordinal data, interval variables with monotonic association. It is robust against outliers, skewed distributions, continuous data and cases where the assumptions of the widely used Pearson's coefficient are violated [55]. On a bivariate dataset it assigns ranks to one variable ignoring the others and vice versa to deduce what is known as the Spearman's rho, denoted by $\mathrm{r}_{\mathrm{s}}$.

The strength of the correlation measured is in the range of $-1 \leq r_{s} \leq 1$, where $r_{\mathrm{s}}$ represents the correlation coefficient [57]. A value of +1 indicates positive monotonic correlation (a perfect positive monotonic relationship), -1 represents negative monotonic correlation (a perfect negative relationship) and 0 represents no monotonic correlation. The closer the value is to +1 or -1 , the stronger the monotonic correlation. Apart from these extreme cases, we need to describe the extent of correlation by a given $r_{s}$ value. Prion and Haerling [56] describe a correlation coefficient in range $[0.81,1.00]$ as very high, range $[0.61,0.80]$ represents a high degree of correlation, range $[0.41,0.60]$ represents a moderate degree of correlation, range $[0.21,0.40]$ indicate a weak correlation and values that lie below 0.29 are regarded as small or negligible correlation.

The outcome of the relationship is grouped under three main categories [58]:

- Positive monotonic correlation - a correlation exists, as a positive increase in one variable causes a proportional increase in the other (or has a tendency to also increase);

- Negative monotonic correlation - for every positive increase in one variable, the other variable decreases or has a tendency to decrease proportionally;

- No correlation-This consist of a random set of values as regardless of any operation done on one variable, the other variable neither tends to increase nor decrease. 


\subsubsection{On Pearson's Correlation Coefficient}

Pearson's Correlation is used to establish a linear relationship on a pair of independent variables. The correlation coefficient is used as a measure of the strength of the relationship given by the Pearson's R. It is usually used as a statistical measure on paired data to identify whether a linear relationship between two datasets exists using a line graph to represent the data. Pearson's correlation attempts to draw a line of best fit based on the data given, to measure the extent to which the homoscedastic (equal variance) pair of independent variable are close to or far from this line. The coefficient, R-value, is generated by dividing the covariance of the two variables by the product of their standard deviations. It should, however, be noted that this value, $\mathrm{R}$, is not the same as the slope of the line in the line graph (or of the line of best fit). Richard Taylor outlines the interpretation of the degree of correlation in [59]: A value of $\mathrm{R}$ in range [0, 0.35] indicates a weak or low correlation, range $[0.26,0.67]$ indicates a moderate correlation, range $[0.68,0.89]$ indicates a strong correlation and range $[0.90,1]$ indicates a very high correlation.

Pearson's correlation requires that the two samples are bivariate [53], [55], normal, and dichotomous [54]. The Pearson's correlation test is sensitive to skewed distributions and outliers [52] and is not appropriate for non-normal and ordinal data as it may then lead to misleading [54] conclusions.

When attempting to use Pearson's coefficient on non-normal data, a transformation approach [78] like the Box Cox, Fisher transformation is required for a set of data that is approximately normal (or close to normal). However, a transformation on an extremely nonnormal data (example with a p-value of 2.2e-16) gives misleading results. The use of the Box Cox transformation, or other data transformation methods to do a monotonic transformation on non-normal data in order to obtain a normal distribution that satisfies the normality assumption of the Pearson's correlation tests, to then allow the use of Pearson's test, though acceptable, may lead to misleading results [55].

\subsubsection{Kendall's Correlation Coefficient}

Kendall's correlation, a non-parametric test measures the statistical association between columns of ranked data. Kendall's coefficient though does better on tied ranks (identical occurrences) than the Spearman's coefficient when the sample size is small and there are not many tied ranks [55]. It also generates narrower confidence intervals. It 
requires ordinal or continuous data and a monotonic relationship to exist between the two variables. Similar to the Spearman's coefficient, it is a distribution-free (assumes no distribution) test of independence.

\subsubsection{Conclusion on correlation tests}

Spearman's correlation test is robust against outliers, skewed distributions, continuous data that is cases where the assumptions of the widely used Pearson's coefficient are violated [55]. Spearman's correlation test is preferred on non-normal data over Pearson's and Kendall's correlation tests due to its robustness against outliers [54] and skewed distributions, at which for instance the Pearson's coefficient fails [52].

For normally distributed datasets (or say transformed non-normal data sets), the Pearson's correlation is ideal [56] for accessing the linear relationship between the two variables while for non-normal data (skewed with outliers), the Spearman correlation is superior as it gives a monotonic relationship between the bivariate datasets, and does not assume any normality or linearity.

Transforming non-normal data sets and then using a Pearson's correlation test is possible when data sets are not extremely non-normal. This may however lead to misleading results. The preservation of the original data and the use of appropriate statistical methods (parametric for normal and non-parametric for non-normal) gives more genuine results, if possible, as sample averages and transformed data most often lead to different results for the same dataset.

A perfect Spearman correlation relates the two variables by a monotonic function whereas the Pearson's Correlation relates them with a linear function.

Spearman's coefficient does better than Kendall's coefficient on tied ranks with large sample sizes and is more sensitive on such samples [55] compared to the results gained using Kendall's coefficient which tends to drift from the desired level as the ties and sample sizes increase [55].

It is also worth noting that the absence of a correlation, say an $r_{s}$-value of 0 , with either of the tests, does not suggest the two variables do not have any relation; it simply means there is no monotonic or linear correlation between the two variables. For instance,

in the case of a quadratic equation such as $f(x)=y=x^{2}$, x and y have a relationship, yet the relation is neither linear nor monotonic and correlation tests return no correlation. 


\subsection{Test for Normality: Anderson Darling Test, Shapiro-Wilk, D’Agostino K- squared, Kolmogorov Smirnov}

When working with statistical methods that assume the data to follow a particular distribution, typically a normal distribution, it is crucial to first perform a hypothesis test for normality (or another distribution) on the given data. The aim is to verify whether the (normality) assumption required for using the other test is satisfied as the validity of many statistical procedures depends on it. As E.S. Pearson [63] puts it: ...It is not enough to know a sample could have come from a normal population; we must be clear that it is at the same time improbable that it has come from a population differing so much from the normal as to invalidate the use of "normal theory" tests in further handling of the material. A parametric test is then chosen for a normal distribution whiles a non-parametric test is for a non-normal distribution. Let us take a look at the various kinds of normality tests.

Common procedures for testing for normality suggested in literature are in the form of graphical methods (histogram, boxplots, QQ plots), numerical methods (skewness and kurtosis indices), and formal methods (Shapiro-Wilk test, Anderson-Darling test, Kolmogorov-Smirnov test, Lilliefors, D'Agosstino skewness test). Though graphical methods suffice for assessing the normality of a given sample, however, they are unreliable as they do not provide sufficient convincing conclusion on whether or not the data is normal [55] for approximately (or almost) non-normal data. A good and complete statistical analysis will include the use of formal, descriptive and inferential statistical methods [62].

In testing for normality, the Anderson-Darling test, a widely used goodness of fit test, is a procedure used to determine whether a sample comes from a specified distribution (Gaussian distribution) for a given number of observations, more precisely, departures from normality in our case. Anderson-Darling is useful for normal, lognormal, Weibull and extreme value type I distributions. However, its counterpart, Shapiro-Wilk is superior, performs better [54], [55], and is used more often since, it exhibits high power and obtains good results for even a small number of observations. This research favors the use of the Shapiro-Wilk test due to its power over all others. D'Agostino's K-squared test is also a good alternative for Shapiro-Wilk but is mostly used in detecting departures from normality and whether a sample has a skewed distribution and kurtosis. D'Agostino and Belanger [61], [62] believe that the power of Kolmogorov Smirnov (Goodness of fit) test in 
determining normality is so low that it should not be considered seriously. On a large study on investigating the power of statistical procedures on normality testing, Shapiro-Wilk emerges as the most powerful and most highly recommended solution [61] [62] [64].

\subsection{Homogeneity of Variance: T-Test and ANOVA}

The t-test is a parametric statistical method to make inferential decisions about the significant difference in the mean values of two variables. The Analysis of Variance (ANOVA) is a t-test equivalence for determining a difference between multiple experimental groups (two or more variables) or running multiple t-tests. In its simplest form, the t-test determines a difference in the mean of two data sets while the ANOVA is for multiple groups. The t-test assumes normality of the groups under comparison, homogeneity of variance (or equal variance). The t-test uses the $t$-statistic, the t-distribution and the degree of freedom to determine the probability value (p-value). For a test conducted on two variables, the t-test and the ANOVA will yield the same results, however, on three or more tests, the compounded effect will lead a t-test to create a Type I error (falsely rejecting a true null hypothesis when it is actually true and proceeding to accept the alternate hypothesis - false positive) [65],[67]. A Type II error occurs when the alternate hypothesis is true and the null hypothesis is falsely accepted when it should have been rejected (false negative). The fundamental principle of ANOVA requires the presence of a parametric dependable variable and multiple independent variables (factors).

The ANOVA test assumes that the subjects are randomly chosen, the response variable is normally distributed, and the population standard deviation is the same for all groups regardless of the differences in mean [67]. ANOVA and t-test are parametric tests and therefore, work very well on normally distributed data. However, with a violation to these normality and equal variance assumptions [65] the results are fairly trustworthy in the absence of non-parametric statistical methods given the sample size is "very large" and the number of subjects in each group are homoscedastic (property of having equal variance or homogeneity of variance). The largeness of a sample cannot be quantified per the central limit theorem [68] [69] and hence the validity of such an assumption requires a population that is not less than $30^{8}$. This will not work for a fixed population whose sample size cannot be further expanded. 
We will not use ANOVA (as we have two variables) and t-test due to the violation of the normality and equal variance assumptions, and the presence of outliers, and skewed distribution. Non-parametric equivalence of ANOVA include the Kruskal-Wallis whilst that of the t-test is the Wilcoxon Rank Sum Test and Kolmogorov Smirnov Test.

\subsection{Statistical Analysis - Wilcoxon Rank Sum Test}

In hypothesis testing, a statistical data is often compared against a statistical synthetic data of an idealized model or a different statistical data and a hypothesis is inferred to confirm the existence of a relationship between the two datasets. A null hypothesis is initially assumed and accepted given the threshold of the significant level (probability value - p-value) is not exceeded. For a p-value that is lesser than the threshold probability (or the alpha significant level), the null hypothesis is rejected for an alternate hypothesis if the comparison reveals that the two datasets are statistically significant. The outcome will be used to infer whether the difference in the two datasets is by chance or statistically significant.

A normality test is initially conducted to establish if the data follows a particular distribution. This determines the choice of statistical method to use: parametric or nonparametric. A parametric statistical method is used for a dataset that follows a normal distribution (Gaussian distribution) while a non-parametric methods are used for a nonnormal data. Among many non-parametric tests that compare the difference in seemingly similar means of two datasets, we have: Mann-Whitney U Test (Wilcoxon Rank Sum Test), Wilcoxon Rank Sign Test, Kruskal-Wallis, Mood's median test, Friedman test and Kolmogorov Smirnov Test.

The Wilcoxon Rank Sign Test requires that two samples be paired (paired variables have paired means with paired measurements). Kruskal-Wallis, which is an extension of the Mann-Whitney U Test [71] is used for three samples. The Wilcoxon Rank Sum Test and the Kolmogorov Smirnov Test [30] assume non-normal, non-parametric, independent and unpaired sets of data. They are the two most powerful non-parametric test to determine the difference in mean values between samples, thereby leading to more confirmatory conclusions. The Kolmogorov Smirnov test easily detects slight changes and shifts in the cumulative distribution of the samples while the Wilcoxon Rank Sum Test is more sensitive to changes in the median value rather than a shift in the cumulative distribution of the 
samples [70]. These two tests and therefore complementary and give a better idea of the results gained from the data.

The Wilcoxon Rank Sum Test (Mann Whitney U Test) tests the median of two distributions with a null hypothesis on the basis that the two distributions are identical. An alternate hypothesis is used to gain evidence [31] against the null hypothesis if the probability value (level of statistical significance) is less than the well-accepted 0.05 level, i.e., with a specified alpha $(\alpha)$ level of $5 \%$. The probability value (p-value) evaluates the probability that the observed results confirms the null hypothesis. A p-value smaller than $\alpha$ is evidence that the alternate hypothesis is true.

\subsubsection{Kolmogorov Smirnov Tests}

The Kolmogorov Smirnov Test (Goodness of Fit for one sample) is a non-parametric statistical method that is used to check for normality (for a single variable against a Gaussian or Laplace-Gauss distribution) or whether two datasets belong to the same distribution. For two sets of non-normal data, it is used to determine whether the two are drawn from the same distribution, by verifying whether there is a difference in the mean values or not. It calculates the maximum difference between the two cumulative distributions and gives a more accurate approximation that the t-test cannot see when detecting differences in a set of data. The Kolmogorov Smirnov Test makes no assumption about the distribution and is robust to the presence of outliers. It does not place a restriction on sample size. It has less power in detecting shifts in the median than the Rank Sum Test but is more powerful at detecting changes and shifts in the shape of the distribution given by the D statistic value. 


\section{Chapter 4 EXPERIMENTAL DESIGN}

In this research, we select a number of software applications, developed with various programming languages and for which we have access to both the application code and the test code. We are interested in analyzing and comparing the complexity of application code and test code to evaluate whether indeed, test code is different to (e.g. is less complex than) application code and at the same time, what is the extent of such a difference (if any). This chapter described our selection of subject program to analyze (section 4.1), our selection of source code metrics (section 4.3), our selection of a software package, and its tailoring, to measure source code (section 4.2), and our statistical hypotheses and analysis (section 4.3).

\subsection{Selected Applications and Rationale}

For our analysis, we considered popular open source applications. These applications have received a credible level of acceptance by millions of users and contributors and have proven to be very useful to users, as evidenced by the number of contributors, commits and downloads. Then we wanted case study software that is (a) publicly accessible and can easily be retrieved for replication, (b) for which we can retrieve the code from the very source organisation, (c) which has a sizable number of commits, pulls and forks, with more than 10k for Application Code (we believed larger projects have a better chance of containing sufficient tests for our study). This if further explained in the threats to valid section (Chapter 6). We also considered criteria that others have identified for the selection of open source software, including perceived quality, user satisfaction [27], or cost [28].

We selected software for which we have access to both test code and application code, to compare one kind of code with the other for a given software, because we assume that both kinds of code for that software would be developed simultaneously (especially if agile methods are used), by approximately the same group of developers, who would therefore tend to program both kinds of code with similar coding principles. We also argue, although this would need to be confirmed, that, as the complexity of the application code increases, the complexity of its test code may also increase and that, therefore test code and application code for the same subject programs are required for our analysis. Last, we argue 
that it would not make sense to compare the application code of one software with the test code of another software.

For the selection of case study software, we proceeded as follows. We first browsed a number of websites like Quora, Stack Overflow, Eclipse Community and Github, looking for the most used and popular open source projects. In doing so, we followed the process that has been identified as being typically used by users of open-source or free software [29]. For the many results we had, we proceeded to their respective websites in search for their source code (application code and test code). We were mainly interested in software that has the following characteristics: first and foremost, we wanted case study software that has both application code and test code and for which we can easily distinguish what is test code and what is application code to facilitate measurement. For instance we put aside Abiword as its distribution does not come with test code. Some applications were initially considered but were not selected in the end because they had test code files mixed with application code files thereby making it difficult to easily distinguish them from each other during measurement: examples are GCC, Linux 2.6, Guava, Columba 1.4, and Atom; With mixed application and test code, there is a higher risk that we consider an application code file when measuring test code and vice versa, which would be a severe threat to the validity of our results. A total of 500 open source software were considered.

In the end, we selected the 40 open source case study software of Table 1 . The table also indicates the version software used and the URL from which the code from was obtained for each case study.

We then needed to distinguish application code from test code. We proceeded further to separate the application code from the test code and run the analyses on each one separately. Most of the packages we analyzed had the application code in the "src" directory while the test code was in a "test" directory, all within the same root directory. We used the Treemap functionality in Understand (which reveals all subfolders of source files in their parent folders at a glance) to observe the files and sub-directories contained in each directory to ensure we were not missing any misplaced file or sub-directory to adequately classify code as test code or application code. 
Table 1. REFERENCES OF CASE STUDY SOFTWARE BEING STUDIED

\begin{tabular}{|c|c|c|c|c|c|}
\hline Case study name & Version & URL & Case Study name & Version & URL \\
\hline Amorok & 2.9 .0 & https://github.com/KDE/amarok & Keepassx & 2.0 .3 & https://github.com/keepassx/keepassx \\
\hline Amule & 1.28 .0 & https://github.com/Microsoft/vscode & Light GBM & 2.2 .1 & https://github.com/Microsoft/LightGBM \\
\hline Apache Ant & 1.7 .0 & https://github.com/curl/curl & Mongo & r4.1.4 & https://github.com/mongodb/mongo \\
\hline Apache Httpd & 2.2 .8 & https://github.com/apache/httpd & MPV Player & 0.29 .1 & https://github.com/mpv-player/mpv \\
\hline Audacity & 2.3 .1 & https://github.com/audacity/audacity & Musescore & 2.3 .2 & https://github.com/musescore/MuseScore \\
\hline Bitcoin & 0.15 .1 & https://github.com/bitcoin/bitcoin & Mypaint & 1.2 .1 & https://github.com/mypaint/mypaint \\
\hline Client & & https://github.com/owncloud/client & NetData & 1.10 .0 & https://github.com/firehol/netdata \\
\hline Curl & 7.56 .0 & & NMap & 7.7 .0 & https://github.com/nmap/nmap \\
\hline Ditaa & 0.11 .0 & https://github.com/stathissideris/ditaa & Notepad ++ (Scintilla 4.1.2) & 7.5 .9 & $\begin{array}{c}\text { https:/github.com/notepad-plus-plus/notepad-plus- } \\
\text { plus/tree/master/scintilla }\end{array}$ \\
\hline Django & 2.1 .2 & https://github.com/django/django & Onion & 0.2 .0 & https://github.com/OnionIoT/source \\
\hline FileZilla & 3.0 .0 & https://github.com/basvodde/filezilla & PDF Creator & 2.3 .0 & $\begin{array}{c}\text { https://github.com/pdfforge/PDFCreator/tree/master/So } \\
\text { urce }\end{array}$ \\
\hline Foundation DB & 6.0 .14 & https://github.com/apple/foundationdb & Powershell & 6.2 .0 & https://github.com/PowerShell/PowerShell \\
\hline Handbrake & 1.1 .2 & https://github.com/HandBrake/HandBrake & Rosegarden & 10.02 & https://github.com/nengxu/rosegarden \\
\hline Hibernate ORM & 5.3.7.Final & https://github.com/hibernate/hibernate-tools & Apache Shiro & 1.4 .0 & https://github.com/apache/shiro \\
\hline Hibernate & 3.3.2.GA & $\begin{array}{c}\text { https://sourceforge.net/projects/hibernate/files/h } \\
\text { ibernate3/3.3.2.GA/ }\end{array}$ & Apache Sqoop & 1.4.7-rc0 & https://github.com/apache/sqoop \\
\hline Inkscape & 0.92 .3 & https://gitlab.com/inkscape/inkscape & Squirrel SQL & 12 & $\begin{array}{l}\text { https://github.com/igorhvr/squirrel- } \\
\text { sql/tree/master/sql12 }\end{array}$ \\
\hline Jfreechart & 1.0 .10 & http://www.jfree.org/jfreechart/download.html & VLC & 3.0 .4 & https://github.com/videolan/vlc-3.0 \\
\hline Jakarta JMeter & 2.3 .2 & http://jmeter.apache.org/svnindex.html & Voldemort & 1.10 .26 & https://github.com/voldemort/voldemort \\
\hline JRuby & 1.4 .0 & http://jruby.org/download & VSCode & 1.28 .2 & https://github.com/Microsoft/vscode \\
\hline & & & NASA WorldWind Java & 2.1 .0 & https://github.com/NASAWorldWind/WorldWindJava \\
\hline
\end{tabular}


We also run a search for all files and folders that had the name "test" in it to reveal all potential test code. We inspected the packages of the source code to verify whether the application code and test code were in distinct directories by observing what is contained in every directory. We searched the individual directories (application code and test code) to see whether or not some test code files sneaked into the application code files and vice versa using the Treemap functionality of the Understand tool: the Treemap allows one to quickly and visually represent and dig into source code. We analysed the code by viewing a select number of source code in each package within Understand to ensure they really were application code or test code. We checked the naming convention used as some files and directory names (packages) suggest the kind of code included in packages or functions: for instance, we search for package/function names containing string "test".

\subsection{Measurement tool}

To maintain consistency between measurements, and given the characteristics of the case study software presented in Table 1, we needed a source code analysis tool that can consistently measure the Cyclomatic Complexity and the Halstead complexity metrics on Java, C\#, C++, Python and C. We evaluated a number of tools based on their support for these programming languages, their ability to run on tens of thousands of LOC, and the number of metrics they collect from the code.

These metrics can easily be extracted automatically with the use of a variety of tools that support a large number of different programming languages. The most frequently used [3] tools like Understand, CKJM, Authors's tool, Metrics 1.3.6, Parasoft have been considered for use in our work and we finally decided to use Understand. Most of the tools considered are suitable for a single programming language and therefore, present a measurement challenge when dealing with an application written in multiple languages. Also, some tools have difficulties to handle large applications, at which Understand performed extremely well.

Scientific Tools Understand (Sci-Tools Understand) [18] fits our needs and appears to be a prime choice by others [3]. It was therefore preferred over other tools like Parasoft's tools, Testwell, Author's tool, Metrics 1.3.6. Some of these others tools, such as Parasoft's tools, were however used, though results are not reported in this thesis, to confirm some of 
the results obtained by using Understand. Understand was used to measure complexity metric values for functions/methods in application code and test code.

In order to collect exactly the information we needed, we developed scripts to execute in Understand. The information produced by Understand is collected in an Excel file. We also developed scripts to anonymize metric data; this was necessary when dealing with industry data in order to maintain confidentiality. All scripts are available in Appendix E .

\subsection{Selected metrics}

In this study we use software metrics to quantify the complexity difference and establish a correlation relation between the different software artefacts. Although we agree in part to seemingly strong correlation between the complexity metrics through the empirical results gained by some authors, we, however, believe that the results differ depending on the kind of code, the kind of metrics and the kind of software one is analysing.

Additionally, the cyclomatic complexity metric was shown to explain software development effort [35], [36], some authors also question the mere definition of these metrics and therefore their use [38].

Despite this debate, recognizing that the Cyclomatic Complexity is one of the most used [3] source code metrics as well as Lines of Code and Halstead Effort. We decided to also use the Halstead complexity metrics [23], because they are considered complementary [3], [37] to the Cyclomatic Complexity and are therefore often used together [37]. These two structural complexity metrics are somewhat strongly correlated but a combination of the two is practically useful for measuring data flow and control flow as illustrated by Yahya and colleagues [37]. Not only would we want to compare application code to test code using these complexity metrics but also, to verify the variable hypotheses on the correlation of these metrics.

One metric is not capable of giving a full perspective of how complex a program can be due to the different objectives achieved by each metric. However, using different metrics that are deemed complementary, popular and have gained a great level of impact in software quality [37] provides better results and less biased conclusions. Plus, since these metrics are often used by practitioners, the results we will obtain will be more directly 
applicable to practitioners' contexts. Other metrics like Lines of Code (LOC), Number of Methods (NOM), and Number of Classes (NCLASS) will improve our analysis.

Other source code metrics could be used, such as those suggested by Henry and Kafura [25]. However, they have different interpretations [23] and it is difficult to find tool support.

We decided to therefore consider the following source code metrics: Lines of Code (LOC), McCabe cyclomatic complexity, Halstead Difficulty, Halstead Effort, Number of Methods (NOM), Number of Classes (NClass), and Maintainability Index which are all supported by Sci-Tools Understand. Apart from Maintainability which has different variants, Sci-Tools Understand preserves the definitions of all metrics as defined in section 3.1, and uses the Model 2 variant of Maintainability index defined in section 3.2.

\subsection{Selection of Statistical Methods}

When comparing test code complexity with application code complexity at the function/method level, we expect to obtain a very large number of data points which should allow us to conduct some statistical analysis. Knowing how software code is written, following coding principles that encourage short functions/methods, we expect to obtain a large number of data points with low complexity values. For instance, it is well recognized that the complexity of object-oriented software essentially comes from interactions between objects rather than complex function/method bodies. This will especially be the case when measuring the cyclomatic complexity of test code if our assumption, as advocated by prominent authors, that test code is limited to simple control flow, appears to hold. When

plotting a frequency distribution with cyclomatic complexity values increasing on the $\mathrm{x}$ axis, we expect to obtain a distribution that is skewed to the left with a possibly long tail of outliers to the right. In other words, we expect to obtain distributions that do not meet the assumptions of a normal distribution [32].

With such skewed and non-normal distributions, we have to resort to non-parametric statistical methods to test whether there is indeed a significant difference in the median values between application code and test code. Non-parametric tests perform better and are more powerful on non-normal data especially for a large size of discrete data [30]. We are also cognisant that with such skewed distributions, the mean values are strongly affected and may not be meaningful. 
In this study, we will use Wilcoxon Rank Sum Test for determining whether two similar values (average complexity) for application code and test code for a given case study are statistically different or drawn from the same distribution. For our study, we define a null and an alternate hypothesis for Wilcoxon Rank Sum Test as follows:

- Null Hypothesis $\left(\mathrm{H}_{0}\right)$ : The median complexity values of application code and test code are the same.

- Alternate Hypothesis $\left(\mathrm{H}_{1}\right)$ : There is a difference between the median complexity values of application code and test code.

We define the null and alternate hypothesis for Kolmogorov Smirnov test as follows.

- $\mathrm{H}_{\mathrm{o}}$ : The two samples of application code complexity and test code complexity values are drawn from the same distribution.

$\mathrm{H}_{1}$ : The two samples of application code complexity and test code complexity values are drawn from different distributions.

Also, on the correlation of software metrics, we will use Spearman's coefficient as our data is not normal and bivariate. We will not use of Pearson's coefficient, as all our dataset are non-normal. 


\section{Chapter 5 RESULTS (ANALYSIS OF DATA)}

In this chapter, we discuss the metric values extracted using SciTools Understand. We start with providing and discussing some descriptive statistics results (Section 5.1) and then discuss inferential statistical results (Section 5.2). We then discuss the use of nonparametric tests (Section 5.3) and finally investigate the correlation between software metrics (Section 5.4).

\subsection{Statistical Analysis of Extracted Data}

We first provide and discuss some descriptive statistics results on Lines of Code (Section 5.1.1) and Cyclomatic complexity (Section 5.1.2). We also analyse the mean values of the data using frequency distribution, box plots and other graphical methods (Section 5.1.3).

\subsubsection{Descriptive statistics on Lines of Code}

Table 2 provides initial information about the application code (AC) and test code (TC) of the case study systems. Columns provide LOC details for each of AC and TC: the total number of LOC, the ratio of application code over test code, and the contribution of different programming languages towards the total LOC values. Last it provides the number of classes in the code (value 0 in case of procedural code), the number of methods in the code and the average size (LOC) of those methods.

The table shows that the selected case study software are developed with various programming languages, sometimes more than one programming language for one software and up to five different programming languages (eg. Foundation DB), with a predominance of $\mathrm{C}++$ followed by $\mathrm{C}$ and then Java. Most of the applications are written in multiple languages. We manually verified and confirmed this was indeed correct output from Understand. It is not uncommon to find software with more one programming language, to achieve specific goals, including: Performance sensitive software and runtime performance favors the use of low level languages (C++, Rust, Go, C); Communicating with external libraries and APIs written in another language (Pearl, Python) and embedding scripting languages and interpreter (like Guile, Lua, Python) help improve the performance to generate faster executables; The need to include legacy code or legacy language. 
For open source subjects, the largest AC is with Mango (2.3M LOC) while the smallest AC is Ditaa (6,568 LOC); the largest TC is with Mango (173,155 LOC) while the smallest TC is MPV Player (155 LOC). For Industry subjects, AC varies between 3,867 LOC and 83,710 LOC while TC varies beween 27 LOC and 233,085 LOC.

Broadly speaking a values of the ratio of application code to test code strictly greater than one tells a different story than a ratio smaller than one; in the former case there is more $\mathrm{AC}$ than TC meaning that every line of TC essentially tests several lines of AC while in the latter case there is more $\mathrm{TC}$ than $\mathrm{AC}$, meaning that each line of $\mathrm{AC}$ is essentially tested by more than one line of TC. When the ratio is strictly greater than one, it varies between 1.17 for PDF Creator and 2917.12 for Audacity, meaning that for each line of TC there is between 1.17 and 2917 lines of AC; one line of TC tests between 1.17 and 2917 lines of AC. Only two open source subjects have a ratio smaller than one: Django (0.58) and Hibernate ORM (0.88); one line of AC is tested on average by 1.72 (Django) and 1.13 (Hibernate ORM) lines of TC. Judging only by this ratio as a coarse-grained evidence of testing effort, we observe a huge variation of testing effort in these open source subjects. In comparison, the ratio is smaller than one for three Industry subjects $(0.30,0.36$ and 0.86$)$ and greater than one for two Industry subjects (11.65 and 14.29); for the former three, one line of AC is tested on average by 1.16, 2.78 and 3.33 lines of TC while for the latter two, one line of TC tests on average 11 or 14 lines of AC. We conclude that results obtained in our study from these open-source case studies may not generalize to Industry software.

The amount of LOC invariably has an effect on the proportion of test cases to be written. Therefore, a ratio of $\mathrm{AC}$ to $\mathrm{TC}$ of 1 or less suggests a thoroughly tested software over one that has a value greater than 1 . While it is possible to predict that a higher amount of test code over application code suggests that for say a single line of application code, there exist two test code lines and therefore, one object seems more complex than the other by a mere observation of the ratios. Judging only by LOC, application code tends to be more complex than test code especially in open source subjects, but not in Industry subjects.

In analysing the table, there are a number of key observations that require a careful attention. We record extreme cases where the size of AC far exceeds the size of TC by a large multiplicand or factor. A critical observation of the amount of methods for these extreme cases also reveal a similar high ratio for AC and TC. Audacity is an example of this 
extreme cases with 2,917.12 times more lines of $\mathrm{AC}$ than $\mathrm{TC}$ and 2,030 times more $\mathrm{AC}$ methods than TC methods. This is followed by MPV Player with 760 times more lines of AC than TC. Other instances include Apache Httpd whose AC is 360 times larger TC, with 203 times more AC methods than TC methods, Inkscape's AC is 213 times larger than its TC (LOC) with 203 times more AC methods than TC methods. Only a few of these objects show these wide differences in the amount of LOC for AC and TC. The LOC ratio for AC to TC (and ratio of AC to TC methods) for other example are: Powershell (148 and 149), Rosegarden (135 and 198), VLC (95 and 100), Shiro (36 and 154), VSCode (45 and 15) and JRuby (33 and 42). This suggests that the lines of code ratio of application code and test code almost invariably, increases or decreases with the AC to TC ratio of the number of methods. The higher the $\mathrm{LOC} \mathrm{AC} / \mathrm{TC}$ ratio, the higher the $\mathrm{NOM} \mathrm{AC} / \mathrm{TC}$ ratio.

Still on the relation between LOC and NOM, another interesting observation from Table 2 is that, some objects appear to have larger amounts of lines of test code than the application code it tests; i.e. with a ratio of $\mathrm{AC}$ to $\mathrm{TC}$ smaller than 1 . An AC to TC ratio lesser than one implies test code has a higher size than application code. These objects still maintain a value for the NOM and LOC ratios (AC/TC). For example: Django has a LOC $\mathrm{AC} / \mathrm{TC}$ ratio of 0.58 , and a NOM AC/TC ratio of 0.65 , Hibernate has a $0.88 \mathrm{AC} / \mathrm{TC} \mathrm{LOC}$ ratio and a 1,17 $\mathrm{AC} / \mathrm{TC} \mathrm{NOM}$ ratio. While our observation suggests that most application code has a larger size than test code, some objects have an opposite ratio. This also implies that, envisioning complexity from a LOC point of view make test code more complex that is assumed. Similar instances that appear to have a very close range of values for the ratios of the amount of test code and production code are PDF creator and Sqoop which report 1.17 for LOC and 2.05 for number of methods, Curl has 1.89 for AC to TC ratio for LOC and 1.4 for number of methods, whilst Sqoop reports 2.0 for LOC ratio of AT to TC and 1.90 for number of methods. In this case, we observe similar LOC complexity levels and a software that seems to have an almost equal amount of test cases as the application. For such, if test code can be that high, then some test code is not that simple as it is assumed and can have an equal or even higher maintainability difficulties.

All other objects seem to relatively have larger ratio of AC/TC LOC and AC/TC number of methods. Audacity and MPV Player will therefore be discussed separately from 
the other objects due to the less amount of test code size and methods, unless otherwise specified. 
Table 2. SIMPLE METRIC VALUES FOR SOFTWARE OBJECTS

\begin{tabular}{|c|c|c|c|c|c|c|c|c|c|c|c|}
\hline Application & \multicolumn{2}{|c|}{ LOC } & $\begin{array}{r}\text { LOC } \\
\text { AC/TC }\end{array}$ & Java & $\mathrm{C}++$ & C & Python & $\mathrm{C \#}$ & NCIASS & NOM & $\begin{array}{r}\text { Method Avg } \\
\text { size }\end{array}$ \\
\hline \multirow{2}{*}{ Amorok } & $\mathrm{AC}$ & 173639 & \multirow{2}{*}{9.91} & & 171327 & 2237 & 75 & & 1203 & 12952 & 13.41 \\
\hline & $\mathrm{TC}$ & 17526 & & & 17526 & & & & 128 & 995 & 17.61 \\
\hline \multirow{2}{*}{ Amule } & $\mathrm{AC}$ & 104106 & \multirow{2}{*}{27.75} & & 100074 & 4032 & & & 404 & 5813 & 17.91 \\
\hline & $\mathrm{TC}$ & 3752 & & & 3752 & & & & 180 & 468 & 8.02 \\
\hline \multirow{2}{*}{ Apache ant } & $\mathrm{AC}$ & 100433 & \multirow[b]{2}{*}{3.53} & 100299 & & & 94 & 40 & 1197 & 9339 & 10.75 \\
\hline & $\mathrm{TC}$ & 28478 & & 28411 & & & 47 & 20 & 392 & 2984 & 9.54 \\
\hline \multirow{2}{*}{ Apache httpd } & $\mathrm{AC}$ & 291321 & \multirow{2}{*}{359.66} & & 7056 & 284153 & 112 & & 0 & 5709 & 51.03 \\
\hline & $\mathrm{TC}$ & 810 & & & & 810 & & & 0 & 28 & 28.93 \\
\hline \multirow{2}{*}{ Audacity } & $\mathrm{AC}$ & 685524 & \multirow{2}{*}{2917.12} & & 291926 & 393598 & & & 2289 & 32490 & 21.10 \\
\hline & $\mathrm{TC}$ & 235 & & & 235 & & & & 2 & 16 & 14.69 \\
\hline \multirow{2}{*}{ Bitcoin } & $\mathrm{AC}$ & 115726 & \multirow{2}{*}{2.79} & 438 & 106564 & 6329 & 2395 & & 708 & 7448 & 15.54 \\
\hline & $\mathrm{TC}$ & 41492 & & & 25528 & & 15964 & & 220 & 2131 & 19.47 \\
\hline \multirow{2}{*}{ Client } & $\mathrm{AC}$ & 108110 & \multirow{2}{*}{13.57} & & 41891 & 65408 & 811 & & 223 & 4597 & 23.52 \\
\hline & TC & 7968 & & & 7664 & 304 & & & 54 & 585 & 13.62 \\
\hline \multirow{2}{*}{ Curl } & $\mathrm{AC}$ & 54253 & \multirow{2}{*}{1.89} & & 4891 & 49362 & & & 0 & 1448 & 37.47 \\
\hline & $\mathrm{TC}$ & 28641 & & & 314 & 13042 & 15285 & & 301 & 981 & 29.20 \\
\hline \multirow{2}{*}{ Ditaa } & $\mathrm{AC}$ & 6568 & \multirow{2}{*}{13.24} & 6568 & & & & & 44 & 587 & 11.19 \\
\hline & $\mathrm{TC}$ & 496 & & 496 & & & & & 6 & 30 & 16.53 \\
\hline \multirow{2}{*}{ Django } & $\mathrm{AC}$ & 91746 & \multirow{2}{*}{0.58} & & & & 91746 & & 1620 & 10379 & 8.84 \\
\hline & $\mathrm{TC}$ & 158125 & & & & & 158125 & & 6544 & 15742 & 10.04 \\
\hline \multirow{2}{*}{ Filezilla } & $\mathrm{AC}$ & 101112 & \multirow{2}{*}{54.92} & & 70538 & 30574 & & & 328 & 4399 & 22.99 \\
\hline & $\mathrm{TC}$ & 1841 & & & 1841 & & & & 4 & 27 & 68.19 \\
\hline Foundion $D P$ & $\mathrm{AC}$ & 214195 & 22325 & 10139 & 130746 & 59542 & 10710 & 3058 & 694 & 14736 & 14.54 \\
\hline FoundationDB & $\mathrm{TC}$ & 959 & 223.35 & & & & 959 & & 6 & 72 & 13.32 \\
\hline H & $\mathrm{AC}$ & 121137 & 0007 & & 13831 & 73210 & 2126 & 31970 & 378 & 6577 & 18.42 \\
\hline Handbrake & $\mathrm{TC}$ & 4316 & 28.07 & & 20 & 4296 & & & 0 & 42 & 102.76 \\
\hline & $\mathrm{AC}$ & 249226 & 204 & 249226 & & & & & 1591 & 14159 & 17.60 \\
\hline Hibernate & $\mathrm{TC}$ & 82106 & 3.04 & 82106 & & & & & 940 & 7196 & 11.41 \\
\hline Hibernate & $\mathrm{AC}$ & 15328 & - & 15328 & & & & & 198 & 1541 & 9.95 \\
\hline ORM & $\mathrm{TC}$ & 17387 & 0.88 & 17387 & & & & & 210 & 1087 & 16 \\
\hline & $\mathrm{AC}$ & 83710 & & & & 83710 & & & 0 & 427 & 196.04 \\
\hline Industry 1 & $\mathrm{TC}$ & 233085 & 0.36 & & & 233085 & & & 0 & 1281 & 181.96 \\
\hline 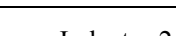 & $\mathrm{AC}$ & 38914 & ser & & & 38914 & & & 0 & 252 & 154.4 \\
\hline Industry 2 & $\mathrm{TC}$ & 88794 & 0.86 & & & 88794 & & & 0 & 538 & 165.04 \\
\hline I 2 & $\mathrm{AC}$ & 44795 & 020 & & & 44795 & & & 0 & 395 & 113.40 \\
\hline Industry 3 & $\mathrm{TC}$ & 144280 & 0.30 & & & 144280 & & & 0 & 743 & 194.18 \\
\hline 1 & $\mathrm{AC}$ & 6036 & 1177 & & & & & 6036 & & 2001 & 3.01 \\
\hline Industry 4 & $\mathrm{TC}$ & 513 & 11.11 & & & & & 513 & & 38 & 13.50 \\
\hline Industry 5 & $\mathrm{AC}$ & 3867 & 143.22 & & & & & 3867 & & 387 & 10 \\
\hline
\end{tabular}




\begin{tabular}{|c|c|c|c|c|c|c|c|c|c|c|c|}
\hline \multirow[t]{2}{*}{ Application } & \multicolumn{2}{|c|}{ LOC } & \multirow{2}{*}{$\begin{array}{r}\text { LOC } \\
\text { AC/TC } \\
\text { Ratio } \\
\end{array}$} & \multirow[t]{2}{*}{ Java } & \multirow[t]{2}{*}{$\mathrm{C}++$} & \multirow[t]{2}{*}{ C } & \multirow[t]{2}{*}{ Python } & \multirow{2}{*}{$\begin{array}{l}\text { C\# } \\
27 \\
\end{array}$} & \multirow[t]{2}{*}{ NCIASS } & \multirow{2}{*}{$\begin{array}{r}\text { NOM } \\
4\end{array}$} & \multirow{2}{*}{$\begin{array}{r}\begin{array}{r}\text { Method Avg } \\
\text { size } \\
\text { (LOC/NOM) }\end{array} \\
6.75 \\
\end{array}$} \\
\hline & TC & 27 & & & & & & & & & \\
\hline \multirow{2}{*}{ Inkscape } & $\mathrm{AC}$ & 488998 & \multirow{2}{*}{213.07} & & 440119 & 46784 & 2095 & & 1529 & 22357 & 21.87 \\
\hline & $\mathrm{TC}$ & 2295 & & & 2295 & & & & 19 & 110 & 20.86 \\
\hline \multirow{2}{*}{ Jfreechart } & $\mathrm{AC}$ & 108979 & \multirow{2}{*}{2.42} & 108979 & & & & & 609 & 7992 & 13.64 \\
\hline & $\mathrm{TC}$ & 44967 & & 44967 & & & & & 399 & 2949 & 15.25 \\
\hline \multirow{2}{*}{ Jkarta JMeter } & $\mathrm{AC}$ & 82062 & \multirow{2}{*}{6.18} & 82062 & & & & & 839 & 7306 & 11.23 \\
\hline & $\mathrm{TC}$ & 13274 & & 13274 & & & & & 111 & 833 & 15.94 \\
\hline \multirow{2}{*}{ JRuby } & $\mathrm{AC}$ & 163076 & \multirow{2}{*}{33.04} & 162814 & & 262 & & & 1977 & 17637 & 9.25 \\
\hline & $\mathrm{TC}$ & 4935 & & 4935 & & & & & 132 & 413 & 11.95 \\
\hline \multirow{2}{*}{ Keepassx } & $\mathrm{AC}$ & 18501 & \multirow{2}{*}{3.85} & & 18439 & & 62 & & 109 & 1295 & 14.29 \\
\hline & $\mathrm{TC}$ & 4808 & & & 4808 & & & & 26 & 172 & 27.95 \\
\hline \multirow{2}{*}{ lightGBM } & $\mathrm{AC}$ & 22612 & 1623 & & 19227 & 4 & 3381 & & 122 & 1393 & 16.23 \\
\hline & TC & 1393 & 10.23 & & & & 1393 & & 6 & 83 & 16.78 \\
\hline Monon & $\mathrm{AC}$ & 2356692 & 1361 & 4969 & 1816201 & 458583 & 76939 & & 11705 & 159337 & 14.79 \\
\hline iviongo & $\mathrm{TC}$ & 173155 & 15.01 & & 1379 & & 171776 & & 40 & 8576 & 20.19 \\
\hline MDV Dloy & $\mathrm{AC}$ & 117809 & 76006 & & 7055 & 108951 & 1803 & & 14 & 5216 & 22.59 \\
\hline MPV Player & $\mathrm{TC}$ & 155 & 100.00 & & & 155 & & & 0 & 24 & 6.46 \\
\hline Musescore & $\mathrm{AC}$ & 593434 & 365 & & 509193 & 77379 & 6862 & & 1626 & 23085 & 25.71 \\
\hline intusescorie & $\mathrm{TC}$ & 162767 & 3.05 & & 162767 & & & & 68 & 963 & 169.02 \\
\hline & $\mathrm{AC}$ & 43993 & & & 5361 & & 38632 & & 378 & 3278 & 13.42 \\
\hline Mypaint & $\mathrm{TC}$ & 1348 & 32.64 & & & & 1348 & & 8 & 96 & 14.04 \\
\hline Netdata & $\mathrm{AC}$ & 48438 & 1658 & & 1849 & 46589 & & & 0 & 1139 & 42.53 \\
\hline Netuata & $\mathrm{TC}$ & 2921 & 10.00 & & & 2921 & & & 0 & 300 & 9.74 \\
\hline & $\mathrm{AC}$ & 213559 & & 45 & 35826 & 156127 & 20950 & 611 & 317 & 6718 & 31.79 \\
\hline NIMap & $\mathrm{TC}$ & 10599 & 20.15 & & 10597 & & & & 0 & 1095 & 20.07 \\
\hline Notenad+t+ & $\mathrm{AC}$ & 77622 & 1483 & & 76401 & 32 & 1189 & & 189 & 3695 & 21.01 \\
\hline Notepadat & TC & 5235 & 14.03 & & 2548 & & 2687 & & 57 & 492 & 10.64 \\
\hline Onion & $\mathrm{AC}$ & 16870 & 4 & & 1640 & 15216 & 14 & & 23 & 1193 & 14.14 \\
\hline Onion & $\mathrm{TC}$ & 4214 & 4 & & 319 & 3895 & & & 2 & 226 & 18.65 \\
\hline Open XML & $\mathrm{AC}$ & 230775 & 108 & & & & & 230775 & 3991 & 58336 & 3.96 \\
\hline SDK & $\mathrm{TC}$ & 116411 & 1.98 & & & & & 116411 & 209 & 1724 & 67.52 \\
\hline & $\mathrm{AC}$ & 45323 & & & & & & 45323 & 1272 & 6564 & 6.90 \\
\hline PDF Creator & $\mathrm{TC}$ & 38754 & 1.17 & & & & & 38754 & 338 & 3188 & 12.16 \\
\hline Dowerchell & $\mathrm{AC}$ & 405873 & 14813 & & 7253 & & 12 & 398608 & 2900 & 29084 & 13.96 \\
\hline Powersnen & TC & 2740 & 140.13 & & & & & 2740 & 47 & 195 & 14.05 \\
\hline Rosegarden & $\mathrm{AC}$ & 169850 & 13502 & & 169850 & & & & 856 & 9905 & 17.15 \\
\hline Nosegaruerr & $\mathrm{TC}$ & 1258 & 135.02 & & 935 & 323 & & & 0 & 50 & 25.16 \\
\hline Shiro & $\mathrm{AC}$ & 37723 & 4584 & 37723 & & & & & 832 & 4267 & 8.84 \\
\hline SnIro & $\mathrm{TC}$ & 823 & 45.84 & 823 & & & & & 9 & 26 & 31.65 \\
\hline & $\mathrm{AC}$ & 83607 & & 83468 & & & 139 & & 841 & 6425 & 13.01 \\
\hline Sqoop & $\mathrm{TC}$ & 41114 & 2.03 & 41114 & & & & & 399 & 3371 & 12.20 \\
\hline
\end{tabular}




\begin{tabular}{|c|c|c|c|c|c|c|c|c|c|c|c|}
\hline Application & \multicolumn{2}{|c|}{ LOC } & $\begin{array}{r}\text { LOC } \\
\text { AC/TC } \\
\text { Ratio }\end{array}$ & Java & $\mathrm{C}++$ & C & Python & $\mathrm{C \#}$ & NCIASS & NOM & $\begin{array}{r}\text { Method Avg } \\
\text { size } \\
\text { (LOC/NOM) }\end{array}$ \\
\hline \multirow{2}{*}{ SQuirrel SQL } & $\mathrm{AC}$ & 194865 & \multirow{2}{*}{7.02} & 194865 & & & & & 3576 & 16706 & 11.66 \\
\hline & $\mathrm{TC}$ & 27762 & & 27762 & & & & & 569 & 3102 & 8.95 \\
\hline \multirow{2}{*}{ VLC } & $\mathrm{AC}$ & 472561 & \multirow{2}{*}{95.45} & & 142794 & 328572 & 1195 & & 806 & 19472 & 24.27 \\
\hline & $\mathrm{TC}$ & 4951 & & & 159 & 4792 & & & 0 & 194 & 25.52 \\
\hline \multirow{2}{*}{ Voldemort } & $\mathrm{AC}$ & 136769 & \multirow{2}{*}{2.69} & 129226 & 2940 & & 4603 & & 1330 & 13148 & 10.40 \\
\hline & $\mathrm{TC}$ & 50837 & & 50764 & & & 73 & & 463 & 2638 & 19.27 \\
\hline \multirow{2}{*}{ VSCode } & $\mathrm{AC}$ & 38681 & \multirow{2}{*}{36.56} & 38537 & 29 & 25 & 74 & 16 & 10 & 1780 & 21.73 \\
\hline & $\mathrm{TC}$ & 1058 & & & & & & 1058 & 2 & 118 & 8.97 \\
\hline \multirow{2}{*}{ Worldwind } & $\mathrm{AC}$ & 329382 & \multirow{2}{*}{21.70} & 324935 & 4447 & & & & 3339 & 26030 & 12.65 \\
\hline & $\mathrm{TC}$ & 15179 & & 15179 & & & & & 107 & 616 & 24.64 \\
\hline
\end{tabular}


This potentially different testing effort between objects means that comparing complexity metric results between objects should be done with care, if at all; indeed an $\mathrm{AC} / \mathrm{TC}$ ratio of cumulative complexity values for one objects may be lower than for another objects simply because the former one may have been more tested than the latter one. Comparing average complexity values should be less sensitive to a potentially different testing effort.

For almost half of the objects, the average size of TC methods is smaller than the average size of AC methods while, it is surprisingly the opposite for the other half. Amorok, Bitcoin, Ditaa, Django, Filezilla, Handbrake, Hibernate ORM, Jakarta JMeter, Mongo, Musescore, Mypaint, NMap, Onion, Open XML, PDF Creator, Powershell, Shiro, VLC, Voldemort, WorldWind Java have a higher average number of methods for AC than TC.

We also note that for pen source objects, the average $\mathrm{AC}$ function size is in range $[3.96,51.03]$ while the average TC function size is in range [6.46, 169.02] while, for Industry objects, the AC average function size is in range $[113,196]$ or $[3,10]$ and the TC average function size is in range $[165,194]$ or $[6.75,13.5]$ (we distinguish between objects Indutry 1-3 on the one hand and objects Industry 4-5 on the other since they look significantly different).

\subsubsection{Descriptive statistics on Cyclomatic Complexity}

Table 3 shows cyclomatic (V(G)) and Halstead (V, D, E, and MI) complexity metric data for AC and TC code for each object as returned by the Understand tool. Data include the cumulative value for the entire ( $\mathrm{AC}$ or $\mathrm{TC}$ ) code, the mean, median, min and max values. The table also includes the ratio of $\mathrm{AC}$ to $\mathrm{TC}$ for Cyclomatic Complexity to measure the extent to which AC is larger or lesser than TC.

All case study software report a higher cumulative Cyclomatic Complexity value for $\mathrm{AC}$ than $\mathrm{TC}$. This is partly due to the size of the objects. $\mathrm{AC}$ is two to hundred times cumulatively more complex than TC: see last column in Table 3. Audacity is an exception: Audacity's AC is 4920 times cumulatively more complex than its $\mathrm{TC}$ for $\mathrm{V}(\mathrm{G})$. Although this may be an artifact of the differences of LOC between AC and TC for these objects (see discussion in previous section), data suggests size is not the only reason: for instance, Audacity's AC is only 2917 times larger than its TC. Application code appears to be more complex that test code. The average cyclomatic complexity values of AC and TC methods 
look very similar: e.g., 2.2 and 1.3 respectively for Apache Ant, 1.5 and 1.2 for Hibernate. Table 3 shows a minimum average Cyclomatic Complexity value of 1.0 (Amorok AC, Light GBM AC and MPV player TC) and a maximum of 16.5 (Handbrake TC). Almost all objects have a higher cumulative cyclomatic complexity for AC than TC with exception to 14 objects where TC is larger.

Although AC appears to be more complex that TC, this does not mean TC is simple. A critical look at the top five average cylomatic complexity values raises an interesting observation. When ranking average cyclomatic complexity values, irrespective of the kind of code (AC or TC), the top two objects exhibit higher TC cyclomatic complexity than AC cyclomatic complexity: for instance, Notepad Plus Plus has a TC cyclomatic complexity value of 14.3 and an AC cyclomatic complexity value of 5.1; on average, a TC method is more complex than a $\mathrm{AC}$ method, although, cumulatively, $\mathrm{AC}$ is 2.7 times more complex than TC. A similar observation is made for Onion: average TC cyclomatic complexity value of 10.7 and an average TC cyclomatic complexity value of 2.9 while the cumulative AC to $\mathrm{TC}$ ratio is 1.45 .

Although $\mathrm{AC}$ has a higher average cyclomatic complexity value than $\mathrm{TC}$ for a majority of the objects, we observe that TC is on the average more complex than AC for 14 objects, with sometimes small differences (e.g., Mypaint) and soetimes larger differences (e.g., Onion): Amorok (1.1 vs. 1.2), Foundation (3.1 vs. 4.0), Light GBM (1.0 vs. 1.6), Mongo (1.1 vs. 2.1), Mypaint (2.5 vs. 2.6), Open XML (1.4 vs. 1.8), Onion (2.9 vs. 10.7), PDF Creator (1.45 vs. 1.48), Rosegarden (0.1 vs. 3.5), Voldemort (1.9 vs. 2.6) and VSCode (2.2 vs. 2.6). This is an indication that some objects have TC methods that are more difficult to understand or maintain than their respective AC methods. With this large amount of objects, it is hard to believe in the hypothesized simplicity of TC.

This is an initial step towards verifying the stated hypothesis that TC is simple. Using typical guidance for using cyclomatic complexity values [5] (Section 3.1), test code in the analyzed systems is in general not simple, nor easy to understand because values tend to be higher than 20 and go up to 2,097. We record a number of instances with a maximum TC cyclomatic complexity larger than 50, which indicates methods that are extremely difficult to maintain. Although not a recommended practice for any kind of source code, observing AC methods with such high complexity values is not entirely surprising at the 
outset; It is however rather surprising to find TC methods with such extremely high values. For example, Notepad has a TC method with a cyclomatic complexity of 2097, a value that is much larger than that of any method in its $\mathrm{AC}$ (the $\mathrm{AC}$ methods have a maximum cyclomatic complexity value of 734). We can make a similar observation for Handbrake and Onion. In other instances, though the maximum cylomatic complexity value of AC methods is larger than that of TC methods, TC methods have reasonably high values: VLC has TC maximum cyclomatic complexity value of 156 while its AC reaches 326; Curl has TC maximum of 97 and AC maximum of 724. If test code methods are, as hypothesized, reduced to a single control flow, to be simple and easy to understand, then the numbers suggest this is not the reality in practice. We observe only a few objects with maximum TC cyclomatic complexity values lesser than 50 . Of all the objects, Hibernate appears as the easiest to maintain with AC methods cyclomatic complexity not exceeding 29 and TC methods cyclomatic complexity values not exceeding 7 .

With a few exceptions, when the median cyclomatic complexity value is larger than one, the code being analyzed is primarily procedural (e.g., C); otherwise, when the code is primarily object-oriented, the median cyclomatic complexity value tends to equal one. This may be due to the larger prevalence of getters and setters, and other simple methods in object-oriented code than in procedural code. The fact that the median and mean cyclomatic complexity values are very often equal to one leads us to conclude that, despite some interesting observations we made earlier, the mean and median values are not very informative as descriptive statistics: there are simply too many methods/functions with a cyclomatic complexity of one. Given the minimum cyclomatic complexity value tends to be one, the maximum cyclomatic complexity value is indicative of the range. The maximum AC cyclomatic complexity value is in range [29, 10019], and in range [29, 734] when we omit outlier Mongo, while the maximum TC cyclomatic complexity value is in range [2, 2097], and in range $[2,196]$ when we omit outlier Notepad++. This suggests that TC tends to be simpler than AC. Cyclomatic Complexity density, a transformation of $\mathrm{CC}$, is a measure of a module's cyclomatic complexity divided by its length (size or LOC). Some authors [80] validate the usefulness of cyclomatic density which has shown to be a useful approximation and predictor of the productivity and difficulty of maintaining code. This metric shows the decision density or how many decisions there are for a given series of lines 
of code. A low cylomatic complexity density predicts a lack of logic while a high cyclomatic density suggest a code dense with decisions. The motivation for this metric is to derive a complexity metric that factors out the size component (thereby normalizing the complexity of the module to better predict difficulty and maintenance productivity) due to the perceived correlation between CC and LOC. In this analysis we observe all objects with a higher cyclomatic complexity density for AC over TC except for 15 objects (Amule, Apache Httpd, Curl, Foundation DB, Hibernate, Industry 2, Industry 5, Jfreechart, LightGBM, Mongo DB, Netdata, Notepad++, Onion, Rosegarden and VSCode).

In this section we conclude that $\mathrm{AC}$ tends to be more complex than $\mathrm{TC}$ but that does not necessarily mean $\mathrm{TC}$ is simple.

Now let's turn our attention to explaining our analysis with detailed Halstead values. Halstead Maintainability Index (MI) also indicates that AC and TC are relatively equally maintainable for each object. There are a few exceptions though. Once again, Audacity reports 7.76, MPV reports 5.22, Shiro reports 5.39, Apache reports 4.37, Ditaa reports 4.23, Foundation reports 2.95, Light GBM reports 2.26. Apache httpd appears to be an outlier: MI value of 4.3 for TC and MI value of .02 for AC. Jakarta JMeter and JRuby are also on that trend, though to a lesser extent.

\subsubsection{Descriptive statistics on Using Distributions, Graphs and Average Values for Cyclomatic Complexity}

Descriptive statistics such as mean and median values do tell a story, as we showed in previous section. However, as we alluded to in these sections, they should be considered with extreme care because they are heavily influenced by the distribution of methods' complexity metric values: for instance, we mentioned that with too many method cyclomatic complexity values of one, mean and median values become less useful. Taking Apache httpd as an example, the average MI value of TC is two orders of magnitude higher than the average MI value of AC. Table 2 tells us that Apache Httpd's LOC for AC is 360 times the TC LOC TC. Assuming standard coding principles are followed, whereby functions are kept simple with a majority of them having a few (to a very few) number of LOC and therefore a low (to a very low) complexity, a much larger number of AC methods may (and do, as confirmed below) artificially lower the average value. 
We therefore need to look at distributions, such as the ones in Appendix A. The appendix provides a few distributions for illustration purposes: frequency distributions of AC and TC method cyclomatic complexity values for Apache httpd and Jfreechart; These two objects have representative distributions of what we obtain for the other objects, though the scales vary. We repeat below the distribution for AC method cyclomatic complexity values as an example. The appendix then includes boxplot of cyclomatic complexity values for AC and TC for all objects, using a logarithmic scale on the y-axis when warranted. (We do not report on the Halstead complexity metric values since the observations we make with cyclomatic complexity, and the conclusions we draw, are overall similar to those obtained with Halstead complexity metrics.) The core features of a box plot are the minimum value, the lower quartile, the median, the upper quartile, the maximum value and the outliers. The distributions are definitely not normal.

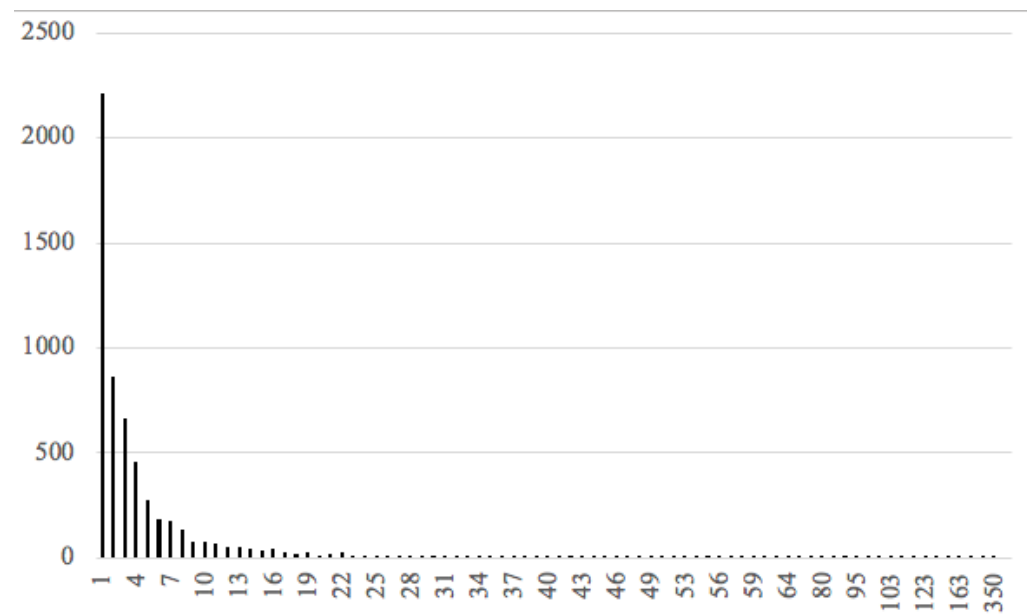

Figure 1. Distribution of cyclomatic complexity values for Apache Httpd AC

A clear observation we can make from the boxplots (Appendix A ) is that the distributions are heavily skewed (to the left) causing the AC and TC boxplots to have the same minimum value, lower quartile, and median in the case of almost all objects.

Apache httpd AC and TC boxplots have the same minimum value and lower quartile, and similar median values ( 2 for AC and 2.5 for TC). Curl's AC and TC boxplots have the same minimum value, a similar lower quartile ( 2 for $\mathrm{AC}$ and 1 for TC), and similar median values ( 3 for $\mathrm{AC}$ and 2 for TC). These distributions, as expected, make descriptive statistics misleading. For instance, without counting Apache httpd Figure 3 test methods with a cyclomatic complexity of one, the mean and median values of test method cyclomatic 
complexity values are 9 and 8, respectively; when counting methods with a cyclomatic complexity of one, these mean and median values are 5.6 and 2.5 , respectively.

We will turn to inferential statistics to make a more convincing conclusions on the median and mean values. 
Table 3. METRIC VALUES (CYCLOMATIC AND HALSTEAD) FOR SOFTWARE OBJECTS

\begin{tabular}{|c|c|c|c|c|c|c|c|c|c|c|c|c|c|}
\hline \multirow{2}{*}{\begin{tabular}{|l} 
Application \\
Amorok
\end{tabular}} & \multirow[b]{2}{*}{$\mathrm{AC}$} & \multicolumn{2}{|c|}{$\mathbf{V}(\mathbf{G})$} & \multicolumn{2}{|c|}{$\mathbf{V}$} & \multicolumn{2}{|c|}{ D } & \multicolumn{2}{|c|}{$\mathbf{E}$} & \multicolumn{2}{|c|}{ MI } & \multirow{6}{*}{\begin{tabular}{|c|} 
CC/LOC \\
0.077240712
\end{tabular}} & \multirow{6}{*}{\begin{tabular}{|c|}
$\mathbf{V}(\mathbf{G}) \mathbf{A C} / \mathbf{T C}$ \\
10.93
\end{tabular}} \\
\hline & & cum. & 13412.0 & cum. & 4052516 & cum. & 98000 & cum. & 84774635 & cum. & 136.31 & & \\
\hline & & mean & 1.0 & mean & 312.9 & mean & 7.6 & mean & 6545.3 & mean & 121.56986 & & \\
\hline & & median & 1.0 & median & 104 & median & 5 & median & 530 & median & 122.32 & & \\
\hline & & $\min$ & 1.0 & $\min$ & 4 & $\min$ & 1 & $\min$ & 4 & $\min$ & 12.67 & & \\
\hline & & $\max$ & 47.0 & $\max$ & 52479 & $\max$ & 1005 & $\max$ & 3645030 & $\max$ & 218.05 & & \\
\hline & \multirow[t]{5}{*}{$\mathrm{TC}$} & cum. & 1227.0 & cum. & 444150 & cum. & 7707 & cum. & 7946986 & cum. & 128.86 & \multirow[t]{5}{*}{0.07001027} & \\
\hline & & mean & 1.2 & mean & 446.4 & mean & 7.7 & mean & 7986.9 & mean & 117.84475 & & \\
\hline & & median & 1.0 & median & 196 & median & 6 & median & 997.5 & median & 117.36 & & \\
\hline & & $\min$ & 1.0 & $\min$ & & $\min$ & 1 & $\min$ & 4 & $\min$ & 45.13 & & \\
\hline & & $\max$ & 14.0 & $\max$ & 63373 & $\max$ & 461 & $\max$ & 1377055 & $\max$ & 204.02 & & \\
\hline \multirow[t]{10}{*}{ Amule } & \multirow[t]{5}{*}{$\mathrm{AC}$} & cum. & 16861.0 & cum. & 4256525 & cum. & 56893 & cum. & 982163092 & cum. & 127.55 & \multirow[t]{5}{*}{0.161959925} & \multirow[t]{10}{*}{17.75} \\
\hline & & mean & 2.9 & mean & 732.2 & mean & 9.8 & mean & 168959.8 & mean & 12408626 & & \\
\hline & & median & 1.0 & median & 116 & median & 6 & median & 688.5 & median & $\begin{array}{r}124.00020 \\
12502\end{array}$ & & \\
\hline & & $\min$ & 1.0 & $\min$ & 4 & $\min$ & 1 & $\min$ & & $\min$ & $\begin{array}{r}125.02 \\
28.08\end{array}$ & & \\
\hline & & $\max$ & 219.0 & $\max$ & 1728260 & $\max$ & 2624 & $\max$ & 898160036 & $\max$ & $\begin{array}{l}-28.08 \\
209.95\end{array}$ & & \\
\hline & \multirow[t]{5}{*}{ TC } & cum. & 950.0 & cum. & 31657 & cum. & 1182 & cum. & 784988 & cum. & 140.83 & \multirow[t]{5}{*}{0.253198294} & \\
\hline & & mean & 2.0 & mean & 67.6 & mean & 2.5 & mean & 1677.3 & mean & 125.59945 & & \\
\hline & & median & 1.0 & median & 64 & median & 5 & median & 288 & median & 127.97 & & \\
\hline & & $\min$ & 1.0 & $\min$ & 4 & $\min$ & 1 & $\min$ & 8 & $\min$ & 58.17 & & \\
\hline & & $\max$ & 55.0 & $\max$ & 24207 & $\max$ & 588 & $\max$ & 753257 & $\max$ & 176.65 & & \\
\hline \multirow[t]{10}{*}{ Apache ant } & \multirow[t]{5}{*}{$\mathrm{AC}$} & cum. & 20093.0 & cum. & 2137071 & cum. & 74957 & cum. & 49237472 & cum. & 148.03 & \multirow[t]{5}{*}{0.200063724} & \multirow[t]{10}{*}{5.12} \\
\hline & & mean & 2.2 & mean & 228.8 & mean & 8.0 & mean & 5772.2 & mean & 14633346 & & \\
\hline & & median & 1.0 & median & 59 & median & 6 & median & 350 & median & 153.425 & & \\
\hline & & $\min$ & 1.0 & $\min$ & & $\min$ & 1 & $\min$ & 4 & $\min$ & & & \\
\hline & & $\max$ & 82.0 & $\max$ & 17836 & $\max$ & 169 & $\max$ & 1105832 & $\max$ & 203.31 & & \\
\hline & \multirow[t]{5}{*}{ TC } & cum. & 3927.0 & cum. & 486696 & cum. & 14799 & cum. & 6453086 & cum. & 154.57 & \multirow[t]{5}{*}{0.13789592} & \\
\hline & & mean & 1.3 & mean & 163.1 & mean & 5.0 & mean & 2162.6 & mean & 133.36249 & & \\
\hline & & median & 1.0 & median & 490.5 & median & 17 & median & 7259 & median & 134.34 & & \\
\hline & & $\min$ & 1.0 & $\min$ & 20 & $\min$ & 2 & $\min$ & 40 & $\min$ & & & \\
\hline & & $\max$ & 21.0 & $\max$ & 11183 & $\max$ & 201 & $\max$ & 376971 & $\max$ & 192.34 & & \\
\hline Apache httpd & $\mathrm{AC}$ & cum. & 28634.0 & cum. & 12181606 & cum. & 187872 & cum. & 80445580 & cum. & 108.68 & 0.098290202 & 180.09 \\
\hline & & mean & 5.0 & mean & 1312.2 & mean & 24.46 & mean & 104733.21 & mean & 106.60536 & & \\
\hline & & median & 2.0 & median & 345 & median & 12 & median & 3942.5 & median & 107.59 & & \\
\hline & & $\min$ & 1.0 & $\min$ & 4 & $\min$ & 1 & $\min$ & & $\min$ & & & \\
\hline & & $\max$ & 350.0 & $\max$ & 914396 & $\max$ & 3250 & $\max$ & 131298137 & $\max$ & 214.1 & & \\
\hline
\end{tabular}




\begin{tabular}{|c|c|c|c|c|c|c|c|c|c|c|c|c|c|}
\hline \multirow[t]{6}{*}{ Application } & \multirow{6}{*}{ TC } & \multicolumn{2}{|c|}{ V(G) } & \multicolumn{2}{|c|}{ V } & \multicolumn{2}{|c|}{ D } & \multicolumn{2}{|c|}{$\mathbf{E}$} & \multicolumn{2}{|c|}{ MI } & \multirow{6}{*}{\begin{tabular}{|c|} 
CC/LOC \\
0.196296296
\end{tabular}} & \multirow[t]{6}{*}{$\mathrm{V}(\mathrm{G}) \mathrm{AC} / \mathrm{TC}$} \\
\hline & & cum. & 159.0 & cum. & 49016 & cum. & 924 & cum. & 1566536 & cum. & 122.38 & & \\
\hline & & mean & 5.7 & mean & 1289.89 & mean & 24.32 & mean & 41224.63 & mean & 107.32333 & & \\
\hline & & median & 2.5 & median & 490.5 & median & 17 & median & 7259 & median & 108.76 & & \\
\hline & & $\min$ & 1.0 & $\min$ & 20 & $\min$ & 2 & $\min$ & & $\min$ & & & \\
\hline & & $\max$ & 25.0 & $\max$ & 11183 & $\max$ & 201 & $\max$ & 376971 & $\max$ & 162.38 & & \\
\hline \multirow[t]{10}{*}{ Audacity } & \multirow[t]{5}{*}{$\mathrm{AC}$} & cum. & 113160.0 & cum. & 18002336 & cum. & 315482 & cum. & $1.231 \mathrm{E}+09$ & cum. & 119.49 & \multirow[t]{5}{*}{0.165070807} & \multirow[t]{10}{*}{4920.00} \\
\hline & & mean & 3.5 & mean & 554.1 & mean & 9.7 & mean & 37884.7 & mean & 115.68196 & & \\
\hline & & median & 2.0 & median & 209 & median & 9 & median & 1818 & median & 115.73 & & \\
\hline & & $\min$ & 1.0 & $\min$ & 4 & $\min$ & 1 & $\min$ & 4 & $\min$ & & & \\
\hline & & $\max$ & 299.0 & $\max$ & 2671128 & $\max$ & 6564 & $\max$ & 451303988 & $\max$ & 209.96 & & \\
\hline & \multirow[t]{5}{*}{ TC } & cum. & 23.0 & cum. & 7713 & cum. & 208 & cum. & 173830 & cum. & 124.14 & \multirow[t]{5}{*}{0.09787234} & \\
\hline & & mean & 1.4 & mean & 482.1 & mean & 13.0 & mean & 10864.4 & mean & 114.255 & & \\
\hline & & median & 1.0 & median & 395.5 & median & 12.5 & median & 6113.5 & median & 112.945 & & \\
\hline & & $\min$ & 1.0 & $\min$ & 28 & $\min$ & 2 & $\min$ & & $\min$ & 95.07 & & \\
\hline & & $\max$ & 3.0 & $\max$ & 4981 & $\max$ & 136 & $\max$ & 128386 & $\max$ & 135.1 & & \\
\hline \multirow[t]{10}{*}{ Bitcoin } & \multirow[t]{5}{*}{$\mathrm{AC}$} & cum. & 20016.0 & cum. & 3841736 & cum. & 176000 & cum. & 328189021 & cum. & 123.5 & \multirow[t]{5}{*}{0.172960268} & \multirow[t]{10}{*}{4.25} \\
\hline & & mean & 2.7 & mean & 879.03 & mean & 19.22 & mean & 35836.32 & mean & 118.43407 & & \\
\hline & & median & 1.0 & median & 173 & median & 9 & median & 1489.5 & median & 118.78 & & \\
\hline & & $\min$ & 1.0 & $\min$ & & $\min$ & 1 & $\min$ & & $\min$ & & & \\
\hline & & $\max$ & 356.0 & $\max$ & 266530 & $\max$ & 2495 & $\max$ & 22947656 & $\max$ & 213.55 & & \\
\hline & \multirow[t]{5}{*}{ TC } & cum. & 4708.0 & cum. & 2980630 & cum. & 47902 & cum. & 159901565 & cum. & 114.99 & \multirow[t]{5}{*}{0.113467656} & \\
\hline & & mean & 2.2 & mean & 1180.92 & mean & 18.98 & mean & 63352.44 & mean & 112.19454 & & \\
\hline & & median & 1.0 & median & 232 & median & 8 & median & 1816 & median & 111.39 & & \\
\hline & & $\min$ & 1.0 & $\min$ & & $\min$ & 1 & $\min$ & 4 & $\min$ & & & \\
\hline & & $\max$ & 45.0 & $\max$ & 91189 & $\max$ & 874 & $\max$ & 10230777 & $\max$ & 176.08 & & \\
\hline Client & $\mathrm{AC}$ & cum. & 13596.0 & cum. & 3792814 & cum. & 68039 & cum. & 255832346 & cum. & 129.57 & 0.125760799 & 17.68 \\
\hline & & mean & 3.0 & mean & 825.1 & mean & 14.8 & mean & 55652.0 & mean & 112.9351 & & \\
\hline & & median & 1.0 & median & 297.5 & median & 11 & median & 3069.5 & median & 113.49 & & \\
\hline & & $\min$ & 1.0 & $\min$ & & $\min$ & & $\min$ & 4 & $\min$ & & & \\
\hline & & $\max$ & 650.0 & $\max$ & 2615241 & $\max$ & 43091 & $\max$ & 227401359 & $\max$ & 211.81 & & \\
\hline & TC & cum. & 769.0 & cum. & 223966 & cum. & 4749 & cum. & 4511580 & cum. & 127.43 & 0.096511044 & \\
\hline & & mean & 1.3 & mean & 382.8 & mean & 8.1 & mean & 7712.1 & mean & 123.36343 & & \\
\hline & & median & 1.0 & median & 171 & median & 6 & median & 1065 & median & 122.32 & & \\
\hline & & $\min$ & 1.0 & $\min$ & & $\min$ & 1 & $\min$ & & $\min$ & 0 & & \\
\hline & & $\max$ & 15.0 & $\max$ & 26026 & $\max$ & 745 & $\max$ & 842105 & $\max$ & 186.18 & & \\
\hline Curl & $\mathrm{AC}$ & cum. & 12270.0 & cum. & 3398918 & cum. & 58432 & cum. & 177821492 & cum. & 116.32 & 0.226162609 & 1.89 \\
\hline & & mean & 8.5 & mean & 1578.69 & mean & 27.14 & mean & 82592.43 & mean & 105.28132 & & \\
\hline & & median & 3.0 & median & 540 & median & 16 & median & 8741.5 & median & 106.66 & & \\
\hline & & $\min$ & 1.0 & $\min$ & 18 & $\min$ & 2 & $\min$ & & $\min$ & 0 & & \\
\hline & & $\max$ & 724.0 & $\max$ & 181049 & $\max$ & 1584 & $\max$ & 12165665 & $\max$ & 163.66 & & \\
\hline
\end{tabular}




\begin{tabular}{|c|c|c|c|c|c|c|c|c|c|c|c|c|c|}
\hline \multirow[t]{6}{*}{ Application } & \multirow{6}{*}{$\mathrm{TC}$} & \multicolumn{2}{|c|}{$V(\mathbf{G})$} & \multicolumn{2}{|r|}{$\mathbf{V}$} & \multicolumn{2}{|c|}{ D } & \multicolumn{2}{|c|}{$\mathbf{E}$} & \multicolumn{2}{|c|}{ MI } & \multirow{6}{*}{\begin{tabular}{|c|} 
CC/LOC \\
0.226318913 \\
\end{tabular}} & \multirow[t]{6}{*}{$\mathrm{V}(\mathrm{G}) \mathrm{AC} / \mathrm{TC}$} \\
\hline & & cum. & 6482.0 & cum. & 1428086 & cum. & 20797 & cum. & 61266955 & cum. & 104.24 & & \\
\hline & & mean & 6.6 & mean & 957.80 & mean & 13.95 & mean & 41091.18 & mean & 105.57034 & & \\
\hline & & median & 2.0 & median & 396.5 & median & 11 & median & 4544.5 & median & 107.085 & & \\
\hline & & $\min$ & 1.0 & $\min$ & 13 & $\min$ & 2 & $\min$ & & $\min$ & & & \\
\hline & & $\max$ & 97.0 & $\max$ & 141013 & $\max$ & 675 & $\max$ & 10043470 & $\max$ & 204.97 & & \\
\hline \multirow[t]{10}{*}{ Ditaa } & \multirow[t]{5}{*}{$\mathrm{AC}$} & cum. & 1596.0 & cum. & 213599 & cum. & 6047 & cum. & 6598707 & cum. & 134.81 & \multirow[t]{5}{*}{0.242996346} & \multirow[t]{10}{*}{31.29} \\
\hline & & mean & 2.7 & mean & 363.9 & mean & 10.3 & mean & 11241.4 & mean & 126.03986 & & \\
\hline & & median & 1.0 & median & 7 & median & 768 & median & 129.29 & median & $\begin{array}{l}129.29 \\
\end{array}$ & & \\
\hline & & $\min$ & 1.0 & $\min$ & 1 & $\min$ & 8 & $\min$ & 28.7 & $\min$ & 28.7 & & \\
\hline & & $\max$ & 98.0 & $\max$ & 1830 & $\max$ & 1888308 & $\max$ & 177.62 & $\max$ & 177.62 & & \\
\hline & \multirow[t]{5}{*}{$\mathrm{TC}$} & cum. & 51.0 & cum. & 18144 & cum. & 365 & cum. & 353391 & cum. & 126.75 & \multirow[t]{5}{*}{0.102822581} & \\
\hline & & mean & 1.7 & mean & 604.8 & mean & 12.2 & mean & 11779.7 & mean & 108.54139 & & \\
\hline & & median & 1.0 & median & 398 & median & 11 & median & 4465 & median & 105.855 & & \\
\hline & & $\min$ & 1.0 & $\min$ & 19 & $\min$ & 2 & $\min$ & & $\min$ & & & \\
\hline & & $\max$ & 7.0 & $\max$ & 3757 & $\max$ & 35 & $\max$ & 131495 & $\max$ & 168.48 & & \\
\hline \multirow[t]{10}{*}{ Django } & \multirow[t]{5}{*}{$\mathrm{AC}$} & cum. & 24343.0 & cum. & 271462676 & cum. & 355331 & cum. & 79076847902 & cum. & 208.98 & \multirow[t]{5}{*}{0.265330369} & \multirow[t]{10}{*}{1.27} \\
\hline & & mean & 2.3 & mean & 26154.99 & mean & 34.24 & mean & 76189.27 & mean & 148.23 & & \\
\hline & & median & 1.0 & median & 186 & median & 9 & median & 1575 & median & 145.32 & & \\
\hline & & $\min$ & 1.0 & $\min$ & & $\min$ & 1 & $\min$ & 8 & $\min$ & & & \\
\hline & & $\max$ & 49.0 & $\max$ & 408866 & $\max$ & 334 & $\max$ & 123987814 & $\max$ & 210.98 & & \\
\hline & \multirow[t]{5}{*}{$\mathrm{TC}$} & cum. & 19200.0 & cum. & 5012126 & cum. & 99042 & cum. & 87621551 & cum. & 200.04 & \multirow[t]{5}{*}{0.121422925} & \\
\hline & & mean & 1.2 & mean & 318.39 & mean & 6.29 & mean & 5566.10 & mean & 187.11 & & \\
\hline & & median & 1.0 & median & 176 & median & 5 & median & 885 & median & 180.91 & & \\
\hline & & $\min$ & 1.0 & $\min$ & & $\min$ & 1 & $\min$ & 8 & $\min$ & & & \\
\hline & & $\max$ & 21.0 & $\max$ & 83359 & $\max$ & 236 & $\max$ & 12503850 & $\max$ & 215.01 & & \\
\hline Filezilla & $\mathrm{AC}$ & cum. & 19714.0 & cum. & 3000079 & cum. & 67804 & cum. & 139380907 & cum. & 111.29 & 0.194971912 & 597.39 \\
\hline & & mean & 4.5 & mean & 682.0 & mean & 15.4 & mean & 31684.7 & mean & 109.34416 & & \\
\hline & & median & 2.0 & median & 259 & median & 11 & median & 2856 & median & 107.13 & & \\
\hline & & $\min$ & 1.0 & $\min$ & & $\min$ & 1 & $\min$ & 4 & $\min$ & -42.04 & & \\
\hline & & $\max$ & 275.0 & $\max$ & 308476 & $\max$ & 3718 & $\max$ & 29590702 & $\max$ & 208.29 & & \\
\hline & $\mathrm{TC}$ & cum. & 33.0 & cum. & 80247 & cum. & 364 & cum. & 2937672 & cum. & 79.74 & 0.017925041 & \\
\hline & & mean & 1.2 & mean & 2972.1 & mean & 13.5 & mean & 108802.7 & mean & 101.70188 & & \\
\hline & & median & 1.0 & median & 1397 & median & 15 & median & 28460 & median & 92.03 & & \\
\hline & & $\min$ & 1.0 & $\min$ & & $\min$ & 1 & $\min$ & & $\min$ & 0 & & \\
\hline & & $\max$ & 3.0 & $\max$ & 40316 & $\max$ & 129 & $\max$ & 2033070 & $\max$ & 159.96 & & \\
\hline FoundationDB & $\mathrm{AC}$ & cum. & 45433.0 & cum. & 14942670 & cum. & 301484 & cum. & 896426808 & cum. & 132.17 & 0.21211046 & 158.86 \\
\hline & & mean & 3.1 & mean & 1219.31 & mean & 24.60 & mean & 73147.84 & mean & 121.13912 & & \\
\hline & & median & 1.0 & median & 168 & median & 8 & median & 1320 & median & 122.59 & & \\
\hline & & $\min$ & 1.0 & $\min$ & & $\min$ & & $\min$ & & $\min$ & -165.43 & & \\
\hline & & $\max$ & 610.0 & $\max$ & 1784413 & $\max$ & 28207 & $\max$ & 189819237 & $\max$ & 213.55 & & \\
\hline
\end{tabular}




\begin{tabular}{|c|c|c|c|c|c|c|c|c|c|c|c|c|c|}
\hline \multirow[t]{6}{*}{ Application } & \multirow{6}{*}{ TC } & \multicolumn{2}{|c|}{$\mathbf{V}(\mathbf{G})$} & \multicolumn{2}{|c|}{$\mathbf{V}$} & \multicolumn{2}{|c|}{ D } & \multicolumn{2}{|c|}{$\mathbf{E}$} & \multicolumn{2}{|c|}{ MI } & \multirow{6}{*}{\begin{tabular}{|c|} 
CC/LOC \\
0.29822732
\end{tabular}} & \multirow[t]{6}{*}{$\mathrm{V}(\mathrm{G}) \mathrm{AC} / \mathrm{TC}$} \\
\hline & & cum. & 286.0 & cum. & 31783 & cum. & 795 & cum. & 867712 & cum. & 199.23 & & \\
\hline & & mean & 4.0 & mean & 441.43 & mean & 66.25 & mean & 12051.56 & mean & 180.89 & & \\
\hline & & median & 2.0 & median & 254 & median & 9 & median & 2340 & median & 180.11 & & \\
\hline & & $\min$ & 1.0 & $\min$ & 15 & $\min$ & 2 & $\min$ & 30 & $\min$ & & & \\
\hline & & $\max$ & 72.0 & $\max$ & 8476 & $\max$ & 58 & $\max$ & 491608 & $\max$ & 210.58 & & \\
\hline \multirow[t]{10}{*}{ Handbrake } & $\mathrm{AC}$ & cum. & 19030.0 & cum. & 2221764 & cum. & 41698 & cum. & 99013610 & cum. & 111.69 & 0.15709486 & \multirow[t]{10}{*}{27.38} \\
\hline & & mean & 2.9 & mean & 337.8 & mean & 6.3 & mean & 15054.5 & mean & 99.50838 & & \\
\hline & & median & 1.0 & median & 415 & median & 13 & median & 5303.5 & median & 99.475 & & \\
\hline & & $\min$ & 1.0 & $\min$ & & $\min$ & 1 & $\min$ & & $\min$ & -10.95 & & \\
\hline & & $\max$ & 136.0 & $\max$ & 147305 & $\max$ & 3323 & $\max$ & 9447824 & $\max$ & 182.88 & & \\
\hline & $\mathrm{TC}$ & cum. & 695.0 & cum. & 134076 & cum. & 1007 & cum. & 7310349 & cum. & 66.37 & 0.16102873 & \\
\hline & & mean & 16.5 & mean & 3192.3 & mean & 24.0 & mean & 174055.9 & mean & 99.140424 & & \\
\hline & & median & 1.0 & median & 64 & median & 4 & median & 248 & median & 99.37 & & \\
\hline & & $\min$ & 1.0 & $\min$ & & $\min$ & 1 & $\min$ & 3 & $\min$ & 0 & & \\
\hline & & $\max$ & 196.0 & $\max$ & 147305 & $\max$ & 3323 & $\max$ & 9447824 & $\max$ & 182.86 & & \\
\hline \multirow[t]{11}{*}{ Hibernate } & $\mathrm{AC}$ & cum. & 21107.0 & cum. & 2461274 & cum. & 86741 & cum. & 412910812 & cum. & 145.75 & 0.084690201 & \multirow[t]{11}{*}{2.55} \\
\hline & & mean & 1.5 & mean & 156.58 & mean & 5.52 & mean & 2626.83 & mean & 131.27441 & & \\
\hline & & median & 1.0 & median & 51 & median & 4 & median & 228 & median & 133.29 & & \\
\hline & & $\min$ & 1.0 & $\min$ & & $\min$ & 1 & $\min$ & 4 & $\min$ & 0 & & \\
\hline & & $\max$ & 29.0 & $\max$ & 14392 & $\max$ & 68 & $\max$ & 978656 & $\max$ & 209.95 & & \\
\hline & $\mathrm{TC}$ & cum. & 8277.0 & cum. & 1724444 & cum. & 42424 & cum. & 33204179 & cum. & 137.31 & 0.100808711 & \\
\hline & & mean & 1.2 & mean & 207.86 & mean & 5.11 & mean & 4002.43 & mean & 134.13833 & & \\
\hline & & median & 1.0 & median & 44 & median & 4 & median & 204 & median & 134.22 & & \\
\hline & & $\min$ & 1.0 & $\min$ & & $\min$ & 1 & $\min$ & 4 & $\min$ & 0 & & \\
\hline & & $\max$ & 7.0 & $\max$ & 20926 & $\max$ & 67 & $\max$ & 1255560 & $\max$ & & & \\
\hline & & & & & & & & & & & 213.55 & & \\
\hline \multirow{10}{*}{$\begin{array}{l}\text { Hibernate } \\
\text { ORM }\end{array}$} & $\mathrm{AC}$ & cum. & 3023.0 & cum. & 406552 & cum. & 12612 & cum. & 8116464 & cum. & 132.43 & 0.197220772 & \multirow[t]{10}{*}{2.51} \\
\hline & & mean & 2.0 & mean & 263.8 & mean & 8.2 & mean & 5267.0 & mean & 12257471 & & \\
\hline & & median & 1.0 & median & 87 & median & 6 & median & 525 & median & 125.56 & & \\
\hline & & $\min$ & 1.0 & $\min$ & & $\min$ & 1 & $\min$ & & $\min$ & & & \\
\hline & & $\max$ & 69.0 & $\max$ & 28134 & $\max$ & 663 & $\max$ & 601542 & $\max$ & 186.61 & & \\
\hline & $\mathrm{TC}$ & cum. & 1202.0 & cum. & 334615 & cum. & 7643 & cum. & 4360900 & cum. & 128.1 & 0.06913211 & \\
\hline & & mean & 1.1 & mean & 307.8 & mean & 7.0 & mean & 4011.9 & mean & 125.84878 & & \\
\hline & & median & 1.0 & median & 83 & median & 6 & median & 518 & median & 130.09 & & \\
\hline & & $\min$ & 1.0 & $\min$ & 4 & $\min$ & 1 & $\min$ & 4 & $\min$ & 0 & & \\
\hline & & $\max$ & 20.0 & $\max$ & 3713 & $\max$ & 28 & $\max$ & 85399 & $\max$ & 208.22 & & \\
\hline \multirow[t]{5}{*}{ Industry 1} & $\mathrm{AC}$ & cum. & 3517.0 & cum. & 1227992 & cum. & 9821 & cum. & 63358007 & cum. & 89.6 & 0.042014096 & \multirow[t]{5}{*}{0.73} \\
\hline & & mean & 8.3 & mean & 2875.8 & mean & 23 & mean & 148379.4 & mean & 86.98 & & \\
\hline & & median & 3.5 & median & 156 & median & 10 & median & 914 & median & 88.56 & & \\
\hline & & $\min$ & 1.0 & $\min$ & & $\min$ & 1 & $\min$ & 4 & $\min$ & 0 & & \\
\hline & & $\max$ & 166.0 & $\max$ & 16968 & $\max$ & 426 & $\max$ & 2002 & $\max$ & 91.51 & & \\
\hline
\end{tabular}




\begin{tabular}{|c|c|c|c|c|c|c|c|c|c|c|c|c|c|}
\hline \multirow[t]{6}{*}{ Application } & \multirow{6}{*}{ TC } & \multicolumn{2}{|c|}{ V(G) } & \multicolumn{2}{|c|}{ V } & \multicolumn{2}{|c|}{ D } & \multicolumn{2}{|c|}{$\mathbf{E}$} & \multicolumn{2}{|c|}{ MI } & \multirow{6}{*}{\begin{tabular}{|c|} 
CC/LOC \\
0.020756376
\end{tabular}} & \multirow[t]{6}{*}{$\mathrm{V}(\mathrm{G}) \mathrm{AC} / \mathrm{TC}$} \\
\hline & & cum. & 4838.0 & cum. & 21456330 & cum. & 45936 & cum. & 6325641126 & cum. & 91.8 & & \\
\hline & & mean & 3.7 & mean & 16749.6 & mean & 35.8 & mean & 4938049.3 & mean & 95.01 & & \\
\hline & & median & 2.0 & median & 181 & median & 17 & median & 1286 & median & 94.11 & & \\
\hline & & $\min$ & 1.0 & $\min$ & & $\min$ & 1 & $\min$ & & $\min$ & & & \\
\hline & & $\max$ & 56.0 & $\max$ & 129459 & $\max$ & 820 & $\max$ & 961878 & $\max$ & 101.42 & & \\
\hline \multirow[t]{10}{*}{ Industry 2} & \multirow[t]{5}{*}{$\mathrm{AC}$} & cum. & 1283.0 & cum. & |410441 & cum. & 4283 & cum. & 31220415 & cum. & 156.18 & \multirow[t]{5}{*}{0.032970139} & \multirow[t]{10}{*}{0.34} \\
\hline & & mean & 5.09 & mean & 1628.73 & mean & 17.00 & mean & 123890.536 & mean & 131.24 & & \\
\hline & & median & 4.0 & median & 88.11 & median & 10 & median & 457 & median & 140.11 & & \\
\hline & & $\min$ & 1.0 & $\min$ & & $\min$ & 1 & $\min$ & 2 & $\min$ & & & \\
\hline & & $\max$ & 144.0 & $\max$ & 1494 & $\max$ & 416 & $\max$ & 1014 & $\max$ & 189.43 & & \\
\hline & \multirow[t]{5}{*}{ TC } & cum. & 3721 & cum. & 8260221 & cum. & 15412 & cum. & 210961817 & cum. & 191.20 & \multirow[t]{5}{*}{0.041905985} & \\
\hline & & mean & 6.91 & mean & 1532.57 & mean & 28.64 & mean & 39213.41 & mean & 201.48 & & \\
\hline & & median & 3.0 & median & 109.95 & median & 18 & median & 613 & median & 199.16 & & \\
\hline & & $\min$ & 1.0 & $\min$ & & $\min$ & 1 & $\min$ & 4 & $\min$ & & & \\
\hline & & $\max$ & 39.0 & $\max$ & 4729.5 & $\max$ & 520 & $\max$ & 81339 & $\max$ & 203.65 & & \\
\hline \multirow[t]{10}{*}{ Industry 3} & \multirow[t]{5}{*}{$\mathrm{AC}$} & cum. & 6858 & cum. & 2,495695 & cum. & 19260 & cum. & 112659215 & cum. & 88.6 & \multirow[t]{5}{*}{0.153097444} & \multirow[t]{10}{*}{0.35} \\
\hline & & mean & 18.4 & mean & 6465.53 & mean & 48.75 & mean & 285213.20 & mean & 93.56 & & \\
\hline & & median & 5.0 & median & 104.18 & median & 8.87 & median & 991.88 & median & 90.22 & & \\
\hline & & $\min$ & 1.0 & $\min$ & & $\min$ & 1 & $\min$ & & $\min$ & & & \\
\hline & & $\max$ & 125.0 & $\max$ & 11312 & $\max$ & 284 & $\max$ & 1334.6667 & $\max$ & 95.49 & & \\
\hline & \multirow[t]{5}{*}{$\mathrm{TC}$} & cum. & $\begin{array}{l}19547.0 \\
\end{array}$ & cum. & 42929054 & cum. & 90688 & cum. & $1.244 \mathrm{e} 10$ & cum. & 89.9 & \multirow[t]{5}{*}{0.135479623} & \\
\hline & & mean & 26.3 & mean & 57778.27 & mean & 122.05 & mean & 167429.34 & mean & 88.41 & & \\
\hline & & median & 2.0 & median & 121.19 & median & 11.88 & median & 858.28 & median & 86.13 & & \\
\hline & & $\min$ & 1.0 & $\min$ & & $\min$ & & $\min$ & & $\min$ & & & \\
\hline & & $\max$ & 25.0 & $\max$ & 86318 & $\max$ & 215.89 & $\max$ & 641252 & $\max$ & 90.16 & & \\
\hline Industry 4 & $\mathrm{AC}$ & cum. & 2510.0 & cum. & 174563 & cum. & 7910 & cum. & 2206746 & cum. & 129.50 & 0.415838304 & 14.59 \\
\hline & & mean & 1.16 & mean & 87.23 & mean & 3.95 & mean & 1102.82 & mean & 120.11 & & \\
\hline & & median & 1.0 & median & 25 & median & 2 & median & 50 & median & 122.32 & & \\
\hline & & $\min$ & 1.0 & $\min$ & 8 & $\min$ & 1 & $\min$ & 8 & $\min$ & & & \\
\hline & & $\max$ & 15.0 & $\max$ & 6477 & $\max$ & 59 & $\max$ & 382143 & $\max$ & 123.96 & & \\
\hline & TC & cum. & 172.0 & cum. & \begin{tabular}{|l|}
20767 \\
\end{tabular} & cum. & 482 & cum. & 234031 & cum. & 127.3 & 0.335282651 & \\
\hline & & mean & 1.19 & mean & 546.50 & mean & 15.06 & mean & 61258.71 & mean & 131.34 & & \\
\hline & & median & 1.0 & median & 156 & median & 7 & median & 1051.5 & median & 130.98 & & \\
\hline & & $\min$ & 1.0 & $\min$ & 19 & $\min$ & 2 & $\min$ & 38 & $\min$ & 0 & & \\
\hline & & $\max$ & 4.0 & $\max$ & 1776 & $\max$ & 21 & $\max$ & 37296 & $\max$ & 146.71 & & \\
\hline Industry 5 & $\mathrm{AC}$ & cum. & 643.0 & cum. & 66779 & cum. & 2612 & cum. & 932435 & cum. & 120.31 & 0.166278769 & 80.36 \\
\hline & & mean & 1.31 & mean & 172.56 & mean & 6.75 & mean & 2409.39 & mean & 130.31 & & \\
\hline & & median & 1.0 & median & 38 & median & 3 & median & 152 & median & 126.65 & & \\
\hline & & $\min$ & 1.0 & $\min$ & 2 & $\min$ & 1 & $\min$ & & $\min$ & 0 & & \\
\hline & & $\max$ & 11.0 & $\max$ & 2502 & $\max$ & 34 & $\max$ & 72558 & $\max$ & 149.22 & & \\
\hline
\end{tabular}




\begin{tabular}{|c|c|c|c|c|c|c|c|c|c|c|c|c|c|}
\hline \multirow{6}{*}{ Application } & \multirow{6}{*}{ TC } & \multicolumn{2}{|c|}{ V(G) } & \multicolumn{2}{|c|}{ V } & \multicolumn{2}{|c|}{ D } & \multicolumn{2}{|c|}{$\mathbf{E}$} & \multicolumn{2}{|c|}{ MI } & \multirow{6}{*}{\begin{tabular}{|c|} 
CC/LOC \\
0.296296296
\end{tabular}} & \multirow{6}{*}{$\mathrm{V}(\mathrm{G}) \mathrm{AC} / \mathrm{TC}$} \\
\hline & & cum. & 8.0 & cum. & 1465 & cum. & 33 & cum. & 14411 & cum. & 100.91 & & \\
\hline & & mean & 2.0 & mean & 366.25 & mean & 8.25 & mean & 3602.75 & mean & 95.23 & & \\
\hline & & median & 1.0 & median & 413 & median & 8 & median & 3538 & median & 98.21 & & \\
\hline & & $\min$ & 1.0 & $\min$ & 108 & $\min$ & 4 & $\min$ & 432 & $\min$ & & & \\
\hline & & $\max$ & 3.0 & $\max$ & 531 & $\max$ & 13 & $\max$ & 6903 & $\max$ & 118.94 & & \\
\hline \multirow[t]{10}{*}{ Inkscape } & \multirow[t]{5}{*}{$\mathrm{AC}$} & cum. & 36227.0 & cum. & 22704752 & cum. & 295251 & cum. & $1.355 \mathrm{E}+09$ & cum. & 120.22 & \multirow[t]{5}{*}{0.074084148} & \multirow[t]{10}{*}{214.36} \\
\hline & & mean & 1.6 & mean & 1015.6 & mean & 13.2 & mean & 60623.3 & mean & 115.58191 & & \\
\hline & & median & 1.0 & median & 225 & median & 9 & median & 2043 & median & 115.82 & & \\
\hline & & $\min$ & 1.0 & $\min$ & 4 & $\min$ & 1 & $\min$ & 4 & $\min$ & -79.98 & & \\
\hline & & $\max$ & 262.0 & $\max$ & 2522698 & $\max$ & 5758 & $\max$ & 269928686 & $\max$ & 220.76 & & \\
\hline & \multirow[t]{5}{*}{ TC } & cum. & 169.0 & cum. & 89202 & cum. & 1182 & cum. & 1522529 & cum. & 121.15 & \multirow[t]{5}{*}{0.073638344} & \\
\hline & & mean & 1.5 & mean & 810.9 & mean & 10.7 & mean & 13841.2 & mean & 113.52039 & & \\
\hline & & median & 1.0 & median & 370 & median & 11 & median & 4004 & median & 116.87 & & \\
\hline & & $\min$ & 1.0 & $\min$ & 18 & $\min$ & 1 & $\min$ & 18 & $\min$ & 38.61 & & \\
\hline & & $\max$ & 5.0 & $\max$ & 27006 & $\max$ & 286 & $\max$ & 433771 & $\max$ & 164.31 & & \\
\hline \multirow[t]{10}{*}{ Jakarta JMeter } & \multirow[t]{5}{*}{$\mathrm{AC}$} & cum. & 12733.0 & cum. & 1575344 & cum. & 48344 & cum. & 27247809 & cum. & 150.02 & \multirow[t]{5}{*}{0.116839024} & \multirow[t]{10}{*}{10.34} \\
\hline & & mean & 1.7 & mean & 195.16 & mean & 5.99 & mean & 3375.60 & mean & 138.18718 & & \\
\hline & & median & 1.0 & median & 59 & median & 5 & median & 264 & median & 135.83 & & \\
\hline & & $\min$ & 1.0 & $\min$ & 4 & $\min$ & 1 & $\min$ & & $\min$ & & & \\
\hline & & $\max$ & 51.0 & $\max$ & 7347 & $\max$ & 78 & $\max$ & 405405 & $\max$ & 207.54 & & \\
\hline & \multirow[t]{5}{*}{$\mathrm{TC}$} & cum. & 1232.0 & cum. & 405256 & cum. & 8450 & cum. & 8498957 & cum. & 130.27 & \multirow[t]{5}{*}{0.02739787} & \\
\hline & & mean & 1.5 & mean & 430.21 & mean & 8.97 & mean & 9022.25 & mean & 119.32421 & & \\
\hline & & median & 1.0 & median & 272 & median & 7 & median & 2133 & median & 120.69 & & \\
\hline & & $\min$ & 1.0 & $\min$ & 8 & $\min$ & 1 & $\min$ & & $\min$ & & & \\
\hline & & $\max$ & 15.0 & $\max$ & 6358 & $\max$ & 69 & $\max$ & 419628 & $\max$ & 185.46 & & \\
\hline Jfreechart & $\mathrm{AC}$ & cum. & 21719.0 & cum. & 2281014 & cum. & 70414 & cum. & 58879690 & cum. & 143.01 & 0.264665741 & 5.49 \\
\hline & & mean & 2.7 & mean & 266.44 & mean & 8.22 & mean & 6877.664992 & mean & 156.01 & & \\
\hline & & median & 1.0 & median & 83 & median & 6 & median & 474 & median & 130.29 & & \\
\hline & & $\min$ & 1.0 & $\min$ & & $\min$ & 1 & $\min$ & & $\min$ & & & \\
\hline & & $\max$ & 51.0 & $\max$ & 17088 & $\max$ & 154 & $\max$ & 1602216 & $\max$ & 181.87 & & \\
\hline & TC & cum. & 3959.0 & cum. & 1348734 & cum. & 30706 & cum. & 29213286 & cum. & 138.77 & 0.298252222 & \\
\hline & & mean & 1.3 & mean & 457.4 & mean & 10.4 & mean & 9906.2 & mean & 120.04 & & \\
\hline & & median & 1.0 & median & 303 & median & 10 & median & 3294 & median & 122.94 & & \\
\hline & & $\min$ & 1.0 & $\min$ & & $\min$ & 1 & $\min$ & 8 & $\min$ & 0 & & \\
\hline & & $\max$ & 21.0 & $\max$ & 14563 & $\max$ & 144 & $\max$ & 1750608 & $\max$ & 201.25 & & \\
\hline JRuby & $\mathrm{AC}$ & cum. & 36397.0 & cum. & 5944927 & cum. & 150964 & cum. & 335833457 & cum. & 138.37 & 0.223190414 & 75.05 \\
\hline & & mean & 2.1 & mean & 302.06 & mean & 7.7 & mean & 17063.84 & mean & 127.05269 & & \\
\hline & & median & 1.0 & median & 114 & median & 6 & median & 684 & median & 129.26 & & \\
\hline & & $\min$ & 1.0 & $\min$ & & $\min$ & 1 & $\min$ & 4 & $\min$ & & & \\
\hline & & $\max$ & 426.0 & $\max$ & 93287 & $\max$ & 296 & $\max$ & 23615635 & $\max$ & 209.33 & & \\
\hline
\end{tabular}




\begin{tabular}{|c|c|c|c|c|c|c|c|c|c|c|c|c|c|}
\hline \multirow[t]{6}{*}{ Application } & \multirow{6}{*}{ TC } & \multicolumn{2}{|c|}{ V(G) } & \multicolumn{2}{|r|}{$\mathbf{V}$} & \multicolumn{2}{|c|}{ D } & \multicolumn{2}{|c|}{$\mathbf{E}$} & \multicolumn{2}{|c|}{ MI } & \multirow{6}{*}{\begin{tabular}{|c|} 
CC/LOC \\
0.098277609
\end{tabular}} & \multirow[t]{6}{*}{$\mathrm{V}(\mathrm{G}) \mathrm{AC} / \mathrm{TC}$} \\
\hline & & cum. & 485.0 & cum. & 72311 & cum. & 2560 & cum. & 895672 & cum. & 151.53 & & \\
\hline & & mean & 1.2 & mean & 134.91 & mean & 6.4 & mean & 1671.023 & mean & 132.95298 & & \\
\hline & & median & 1.0 & median & 72 & median & 5 & median & 360 & median & 133.535 & & \\
\hline & & $\min$ & 1.0 & $\min$ & & $\min$ & 1 & $\min$ & & $\min$ & & & \\
\hline & & $\max$ & 9.0 & $\max$ & 2041 & $\max$ & 36 & $\max$ & 63271 & $\max$ & 204.41 & & \\
\hline \multirow[t]{10}{*}{ Keepassx } & $\mathrm{AC}$ & cum. & 3054.0 & cum. & 427823 & cum. & 11550 & cum. & 8565093 & cum. & 129.93 & 0.165072158 & \multirow[t]{10}{*}{13.11} \\
\hline & & mean & 2.4 & mean & 330.4 & mean & 8.9 & mean & 6614.0 & mean & 113.69981 & & \\
\hline & & median & 1.0 & median & 146 & median & 7 & median & 1060 & median & 116.335 & & \\
\hline & & $\min$ & 1.0 & $\min$ & 10 & $\min$ & 2 & $\min$ & 22 & $\min$ & 31.3 & & \\
\hline & & $\max$ & 48.0 & $\max$ & 28602 & $\max$ & 584 & $\max$ & 992609 & $\max$ & $208.29^{\circ}$ & & \\
\hline & $\mathrm{TC}$ & cum. & 233.0 & cum. & 173548 & cum. & 2590 & cum. & 4460251 & cum. & 115.26 & 0.048460899 & \\
\hline & & mean & 1.4 & mean & 1009.0 & mean & 15.1 & mean & 25931.7 & mean & 99.192589 & & \\
\hline & & median & 1.0 & median & 698 & median & 13 & median & 8620 & median & 100.29 & & \\
\hline & & $\min$ & 1.0 & $\min$ & 25 & $\min$ & 2 & $\min$ & 50 & $\min$ & & & \\
\hline & & $\max$ & 11.0 & $\max$ & 25969 & $\max$ & 501 & $\max$ & 795401 & $\max$ & 147.85 & & \\
\hline \multirow[t]{10}{*}{ lightGBM } & $\mathrm{AC}$ & cum. & 1335.0 & cum. & 680152 & cum. & 18672 & cum. & 27801759 & cum. & 129.32 & 0.059039448 & \multirow[t]{10}{*}{9.96} \\
\hline & & mean & 1.0 & mean & 488.3 & mean & 13.4 & mean & 19958.2 & mean & 116.96415 & & \\
\hline & & median & 2.0 & median & 219 & median & 10 & median & 2273 & median & 114.675 & & \\
\hline & & $\min$ & 1.0 & $\min$ & & $\min$ & 1 & $\min$ & & $\min$ & 20.52 & & \\
\hline & & $\max$ & 35.0 & $\max$ & 48016 & $\max$ & 1226 & $\max$ & 3249235 & $\max$ & 205.86 & & \\
\hline & TC & cum. & 134.0 & cum. & 70404 & cum. & 868 & cum. & 1005732 & cum. & 187.98 & 0.096195262 & \\
\hline & & mean & 1.6 & mean & 848.24 & mean & 10.46 & mean & 12117.25 & mean & 184.32 & & \\
\hline & & median & 1.0 & median & 682 & median & 9 & median & 6528 & median & 180.93 & & \\
\hline & & $\min$ & 1.0 & $\min$ & 30 & $\min$ & 2 & $\min$ & 60 & $\min$ & 0 & & \\
\hline & & $\max$ & 6.0 & $\max$ & 2962 & $\max$ & 26 & $\max$ & 70392 & $\max$ & 201.88 & & \\
\hline \multirow[t]{10}{*}{ Mongo } & $\mathrm{AC}$ & cum. & 182531.0 & cum. & 167907982 & cum. & 2517212 & cum. & 6450634454 & cum. & 135.45 & 0.077452208 & \multirow[t]{10}{*}{10.02} \\
\hline & & mean & 1.1 & mean & 1232.84 & mean & 18.49 & mean & 47375.39993 & mean & 121.09176 & & \\
\hline & & median & 1.0 & median & 155 & median & 7 & median & 994 & median & 121.95 & & \\
\hline & & $\min$ & 1.0 & $\min$ & 4 & $\min$ & & $\min$ & 4 & $\min$ & -2374.91 & & \\
\hline & & $\max$ & 10019.0 & $\max$ & 16497738 & $\max$ & 42166 & $\max$ & 606572002 & $\max$ & 213.55 & & \\
\hline & TC & cum. & 18211.0 & cum. & 9587395 & cum. & 112195 & cum. & 417705460 & cum. & 145.77 & 0.105171667 & \\
\hline & & mean & 2.1 & mean & 978.71 & mean & 11.45 & mean & 42640.41 & mean & 127.14983 & & \\
\hline & & median & 1.0 & median & 191 & median & 7 & median & 1266 & median & 129.68 & & \\
\hline & & $\min$ & 1.0 & $\min$ & 8 & $\min$ & 1 & $\min$ & 8 & $\min$ & & & \\
\hline & & $\max$ & 83.0 & $\max$ & 129459 & $\max$ & 320 & $\max$ & 39096618 & $\max$ & 177.39 & & \\
\hline \multirow[t]{5}{*}{ MPV Player } & $\mathrm{AC}$ & cum. & 23190.0 & cum. & 3592760 & cum. & 84254 & cum. & 113009019 & cum. & 116.02 & 0.196844044 & \multirow[t]{5}{*}{927.60} \\
\hline & & mean & 4.4 & mean & 688.8 & mean & 16.2 & mean & 21665.8 & mean & 102.73209 & & \\
\hline & & median & 2.0 & median & 381.5 & median & 14 & median & 5466 & median & 104.04 & & \\
\hline & & $\min$ & 1.0 & $\min$ & 10 & $\min$ & 1 & $\min$ & & $\min$ & & & \\
\hline & & $\max$ & 107.0 & $\max$ & 168981 & $\max$ & 4507 & $\max$ & 4992074 & $\max$ & 178.16 & & \\
\hline
\end{tabular}




\begin{tabular}{|c|c|c|c|c|c|c|c|c|c|c|c|c|c|}
\hline \multirow{6}{*}{ Application } & \multirow{6}{*}{ TC } & \multicolumn{2}{|c|}{$V(G)$} & \multicolumn{2}{|c|}{$\mathbf{V}$} & \multicolumn{2}{|c|}{ D } & \multicolumn{2}{|c|}{ E } & \multicolumn{2}{|c|}{ MI } & \multirow{6}{*}{\begin{tabular}{|c|} 
CC/LOC \\
0.161290323
\end{tabular}} & \multirow[t]{6}{*}{$\mathrm{V}(\mathrm{G}) \mathrm{AC} / \mathrm{TC}$} \\
\hline & & cum. & 25.0 & cum. & 4400 & cum. & 155 & cum. & 44198 & cum. & 125.39 & & \\
\hline & & mean & 1.0 & mean & 183.3 & mean & 6.5 & mean & 1841.6 & mean & 120.40815 & & \\
\hline & & median & 3.0 & median & 110 & median & 5 & median & 675 & median & 126.04 & & \\
\hline & & $\min$ & 1.0 & $\min$ & 70 & $\min$ & 3 & $\min$ & 280 & $\min$ & & & \\
\hline & & $\max$ & 2.0 & $\max$ & 3062 & $\max$ & 103 & $\max$ & 33630 & $\max$ & 141.48 & & \\
\hline \multirow[t]{10}{*}{ Musescore } & \multirow[t]{5}{*}{$\mathrm{AC}$} & cum. & 41760.0 & cum. & 13778244 & cum. & 273861 & cum. & 687739924 & cum. & 123.72 & \multirow[t]{5}{*}{0.070370083} & \multirow[t]{10}{*}{39.47} \\
\hline & & mean & 1.8 & mean & 596.8 & mean & & mean & & mean & 120.54783 & & \\
\hline & & median & 1.0 & median & 140 & median & 7 & median & 938 & median & 120.55 & & \\
\hline & & $\min$ & 1.0 & $\min$ & & $\min$ & 1 & $\min$ & 4 & $\min$ & -48.81 & & \\
\hline & & $\max$ & 243.0 & $\max$ & 246951 & $\max$ & 5635 & $\max$ & 30432620 & $\max$ & 213.02 & & \\
\hline & \multirow[t]{5}{*}{$\mathrm{TC}$} & cum. & 1058.0 & cum. & 291451 & cum. & 6287 & cum. & 7681767 & cum. & 116.44 & \multirow[t]{5}{*}{0.006500089} & \\
\hline & & mean & 1.1 & mean & 302.6 & mean & 11.9 & mean & 29791.6 & mean & 136.40204 & & \\
\hline & & median & 1.0 & median & 31 & median & 2 & median & 62 & median & 152.91 & & \\
\hline & & $\min$ & 1.0 & $\min$ & & $\min$ & 1 & $\min$ & & $\min$ & & & \\
\hline & & $\max$ & 13.0 & $\max$ & 41006 & $\max$ & 915 & $\max$ & 1783268 & $\max$ & 196.13 & & \\
\hline \multirow[t]{10}{*}{ Mypaint } & \multirow[t]{5}{*}{$\mathrm{AC}$} & cum. & 8177.0 & cum. & 82138 & cum. & 1831 & cum. & 3475597 & cum. & 124.43 & \multirow[t]{5}{*}{0.185870479} & \multirow[t]{10}{*}{32.97} \\
\hline & & mean & 2.5 & mean & 25.1 & mean & & mean & & mean & 113.11175 & & \\
\hline & & median & 1.0 & median & 261 & median & 10 & median & 2934 & median & 114.3 & & \\
\hline & & $\min$ & 1.0 & $\min$ & 11 & $\min$ & 2 & $\min$ & & $\min$ & & & \\
\hline & & $\max$ & 54.0 & $\max$ & 20917 & $\max$ & 525 & $\max$ & 878846 & $\max$ & 176.85 & & \\
\hline & \multirow[t]{5}{*}{$\mathrm{TC}$} & cum. & 248.0 & cum. & 31728 & cum. & 671 & cum. & 550006 & cum. & 197.5 & \multirow[t]{5}{*}{0.183976261} & \\
\hline & & mean & 2.6 & mean & 330.5 & mean & 6.99 & mean & 5729.22 & mean & 190.04 & & \\
\hline & & median & 1.0 & median & 125.5 & median & 4 & median & 498 & median & 181.90 & & \\
\hline & & $\min$ & 1.0 & $\min$ & 15 & $\min$ & 2 & $\min$ & 30 & $\min$ & & & \\
\hline & & $\max$ & 21.0 & $\max$ & 3821 & $\max$ & 33 & $\max$ & 126093 & $\max$ & 218.98 & & \\
\hline Netdata & $\mathrm{AC}$ & cum. & 8831.0 & cum. & 1927370 & cum. & 25188 & cum. & 107931209 & cum. & 93.91 & 0.182315537 & 12.54 \\
\hline & & mean & & mean & 1692.2 & mean & 22.1 & mean & 94759.6 & mean & 88.25 & & \\
\hline & & median & 3.0 & median & 565 & median & 18 & median & 9834 & median & 80.17 & & \\
\hline & & $\min$ & 1.0 & $\min$ & 13 & $\min$ & 2 & $\min$ & & $\min$ & $\begin{array}{r}00.17 \\
0\end{array}$ & & \\
\hline & & $\max$ & 231.0 & $\max$ & 97647 & $\max$ & 1705 & $\max$ & 5922011 & $\max$ & 135.18 & & \\
\hline & $\mathrm{TC}$ & cum. & 704.0 & cum. & 64508 & cum. & 1264 & cum. & 2606101 & cum. & 107.37 & 0.241013352 & \\
\hline & & mean & 2.3 & mean & 215.0 & mean & 4.2 & mean & 8687.0 & mean & 98.479077 & & \\
\hline & & median & 1.0 & median & 203.5 & median & 6 & median & 1317 & median & 93.08 & & \\
\hline & & $\min$ & 1.0 & $\min$ & 19 & $\min$ & 2 & $\min$ & & $\min$ & 0 & & \\
\hline & & $\max$ & 41.0 & $\max$ & 33350 & $\max$ & 359 & $\max$ & 3435050 & $\max$ & 166.14 & & \\
\hline NMap & $\mathrm{AC}$ & cum. & 30729.0 & cum. & 4030415 & cum. & 80605 & cum. & 252923142 & cum. & 122.93 & 0.143889979 & 23.57 \\
\hline & & mean & 4.7 & mean & 614.2 & mean & 12.3 & mean & 38543.6 & mean & 110.42934 & & \\
\hline & & median & 1.0 & median & 243 & median & 10 & median & 2329.5 & median & 113.16 & & \\
\hline & & $\min$ & 1.0 & $\min$ & & $\min$ & 1 & $\min$ & & $\min$ & & & \\
\hline & & $\max$ & 54.0 & $\max$ & 169656 & $\max$ & 2540 & $\max$ & 37851011 & $\max$ & 184.96 & & \\
\hline
\end{tabular}




\begin{tabular}{|c|c|c|c|c|c|c|c|c|c|c|c|c|c|}
\hline \multirow{6}{*}{ Application } & \multirow{6}{*}{ TC } & \multicolumn{2}{|c|}{ V(G) } & \multicolumn{2}{|c|}{ V } & \multicolumn{2}{|c|}{ D } & \multicolumn{2}{|c|}{$\mathbf{E}$} & \multicolumn{2}{|c|}{ MI } & \multirow{6}{*}{\begin{tabular}{|c|} 
CC/LOC \\
0.123030475
\end{tabular}} & \multirow[t]{6}{*}{$\mathrm{V}(\mathrm{G}) \mathrm{AC} / \mathrm{TC}$} \\
\hline & & cum. & 1304.0 & cum. & 200199 & cum. & 5118 & cum. & 73192170 & cum. & 122.52 & & \\
\hline & & mean & 1.03 & mean & 158.9 & mean & 4.1 & mean & 58089.0 & mean & 101.9364 & & \\
\hline & & median & 2.0 & median & 462.5 & median & 16.5 & median & 7648.5 & median & 102.81 & & \\
\hline & & $\min$ & 1.0 & $\min$ & 38 & $\min$ & 2 & $\min$ & & $\min$ & & & \\
\hline & & $\max$ & 21.0 & $\max$ & 19574 & $\max$ & 508 & $\max$ & 891667 & $\max$ & 153.49 & & \\
\hline \multirow[t]{10}{*}{ Notepad ++ } & $\mathrm{AC}$ & cum. & 18926.0 & cum. & 2565949 & cum. & 55233 & cum. & 186462571 & cum. & 111.84 & 0.243822628 & \multirow[t]{10}{*}{2.70} \\
\hline & & mean & 5.1 & mean & 694.4 & mean & 14.9 & mean & 50463.5 & mean & 114.95206 & & \\
\hline & & median & 1.0 & median & 162 & median & 9 & median & 1452 & median & 119.13 & & \\
\hline & & $\min$ & 1.0 & $\min$ & 4 & $\min$ & 1 & $\min$ & 4 & $\min$ & -180.25 & & \\
\hline & & $\max$ & 734.0 & $\max$ & 270039 & $\max$ & 3629 & $\max$ & 22365037 & $\max$ & 205.45 & & \\
\hline & TC & cum. & 7019.0 & cum. & 71095 & cum. & 1357 & cum. & 10736465 & cum. & 139.55 & 1.34078319 & \\
\hline & & mean & 14.3 & mean & 144.5 & mean & 2.8 & mean & 21822.1 & mean & 145.58088 & & \\
\hline & & median & 1.0 & median & 128 & median & 6 & median & 869 & median & $\begin{array}{r}14.00000 \\
150.43\end{array}$ & & \\
\hline & & $\min$ & 1.0 & $\min$ & 4 & $\min$ & 1 & $\min$ & 4 & $\min$ & & & \\
\hline & & $\max$ & 2097.0 & $\max$ & 12386 & $\max$ & 915 & $\max$ & 1213828 & $\max$ & 167.33 & & \\
\hline \multirow[t]{10}{*}{ Onion } & $\mathrm{AC}$ & cum. & 3499.0 & cum. & 305509 & cum. & 8110 & cum. & 7948880 & cum. & 137.31 & 0.207409603 & \multirow[t]{10}{*}{1.45} \\
\hline & & mean & 2.9 & mean & 256.1 & mean & 6.8 & mean & 6662.9 & mean & 116.69433 & & \\
\hline & & median & 2.0 & median & 230 & median & 9 & median & 2004 & median & 116.58 & & \\
\hline & & $\min$ & 1.0 & $\min$ & & $\min$ & 1 & $\min$ & & $\min$ & 52.85 & & \\
\hline & & $\max$ & 38.0 & $\max$ & 27724 & $\max$ & 747 & $\max$ & 836053 & $\max$ & 186.37 & & \\
\hline & \begin{tabular}{|l|}
$\mathrm{TC}$ \\
\end{tabular} & cum. & 2420.0 & cum. & 132535 & cum. & 2255 & cum. & 2156004 & cum. & 119.6 & 0.574276222 & \\
\hline & & mean & 10.7 & mean & 586.4 & mean & 10.0 & mean & 9539.8 & mean & 102.55605 & & \\
\hline & & median & 6.0 & median & 471 & median & 9 & median & 4400 & median & 101.16 & & \\
\hline & & $\min$ & 1.0 & $\min$ & 22 & $\min$ & 2 & $\min$ & 44 & $\min$ & & & \\
\hline & & $\max$ & 69.0 & $\max$ & 17071 & $\max$ & 316 & $\max$ & 343244 & $\max$ & 170.34 & & \\
\hline \multirow{10}{*}{$\begin{array}{l}\text { Opn XML } \\
\text { SDK }\end{array}$} & $\mathrm{AC}$ & cum. & 82410.0 & cum. & 3294512 & cum. & 144735 & cum. & 128258645 & cum. & 219.37 & 0.357101072 & \multirow[t]{10}{*}{26.47} \\
\hline & & mean & 1.4 & mean & 56.47 & mean & 2.48 & mean & 2198.62 & mean & 190.03 & & \\
\hline & & median & 1.0 & median & 38 & median & 3 & median & 114 & median & 189.22 & & \\
\hline & & $\min$ & 1.0 & $\min$ & & $\min$ & 1 & $\min$ & 4 & $\min$ & & & \\
\hline & & $\max$ & 245.0 & $\max$ & 433126 & $\max$ & 224 & $\max$ & 97020224 & $\max$ & 219.90 & & \\
\hline & TC & cum. & 3113.0 & cum. & 9164703 & cum. & 28682 & cum. & 363954560 & cum. & 186.25 & 0.026741459 & \\
\hline & & mean & 1.8 & mean & 5315.95 & mean & 16.64 & mean & 21213.87 & mean & 184.75 & & \\
\hline & & median & 1.0 & median & 162 & median & 7 & median & 1141 & median & 180.11 & & \\
\hline & & $\min$ & 1.0 & $\min$ & & $\min$ & 1 & $\min$ & 8 & $\min$ & 0 & & \\
\hline & & $\max$ & 23.0 & $\max$ & 618694 & $\max$ & 474 & $\max$ & 90501768 & $\max$ & 215.76 & & \\
\hline \multirow[t]{5}{*}{ PDF Creator } & $\mathrm{AC}$ & cum. & 9520.0 & cum. & 842457 & cum. & 33622 & cum. & 10400228 & cum. & 195.57 & 0.210047879 & \multirow[t]{5}{*}{2.01} \\
\hline & & mean & 1.5 & mean & 128.35 & mean & 5.122 & mean & 1584.43 & mean & 199.03 & & \\
\hline & & median & 1.0 & median & 39 & median & 3 & median & 117 & median & 185.86 & & \\
\hline & & $\min$ & 1.0 & $\min$ & 2 & $\min$ & 1 & $\min$ & & $\min$ & & & \\
\hline & & $\max$ & 38.0 & $\max$ & 9984 & $\max$ & 94 & $\max$ & 253048 & $\max$ & 214.28 & & \\
\hline
\end{tabular}




\begin{tabular}{|c|c|c|c|c|c|c|c|c|c|c|c|c|c|}
\hline \multirow[t]{6}{*}{ Application } & \multirow{6}{*}{ TC } & \multicolumn{2}{|c|}{$\mathbf{V}(\mathbf{G})$} & \multicolumn{2}{|c|}{$\mathbf{V}$} & \multicolumn{2}{|c|}{ D } & \multicolumn{2}{|c|}{$\mathbf{E}$} & \multicolumn{2}{|c|}{ MI } & \multirow{6}{*}{\begin{tabular}{|c|} 
CC/LOC \\
0.122516385
\end{tabular}} & \multirow[t]{6}{*}{$\mathrm{V}(\mathrm{G}) \mathrm{AC} / \mathrm{TC}$} \\
\hline & & cum. & 4748.0 & cum. & 1175551 & cum. & 27689 & cum. & 13266858 & cum. & 186.25 & & \\
\hline & & mean & 1.5 & mean & 368.72 & mean & 8.69 & mean & 7298.26 & mean & 189.06 & & \\
\hline & & median & 1.0 & median & 142 & median & 5 & median & 732 & median & 180.51 & & \\
\hline & & $\min$ & 1.0 & $\min$ & 8 & $\min$ & 1 & $\min$ & 8 & $\min$ & 0 & & \\
\hline & & $\max$ & 28.0 & $\max$ & 4764 & $\max$ & 57 & $\max$ & 271548 & $\max$ & 200.16 & & \\
\hline \multirow[t]{10}{*}{ Powershell } & $\mathrm{AC}$ & cum. & 81489.0 & cum. & 117745 & cum. & 2900 & cum. & 3539483 & cum. & 126.99 & 0.200774627 & \multirow[t]{10}{*}{270.73} \\
\hline & & mean & 2.8 & mean & 4.0 & mean & 0.1 & mean & 121.7 & mean & 116.9978 & & \\
\hline & & median & 1.0 & median & 22 & median & 180 & median & 7 & median & 117.035 & & \\
\hline & & $\min$ & 1.0 & $\min$ & 3 & $\min$ & 4 & $\min$ & 1 & $\min$ & & & \\
\hline & & $\max$ & 129.0 & $\max$ & 1177 & $\max$ & 32159 & $\max$ & 512 & $\max$ & 205.47 & & \\
\hline & TC & cum. & 301.0 & cum. & 69848 & cum. & 1602 & cum. & 1805272 & cum. & 187.98 & 0.109854015 & \\
\hline & & mean & 1.8 & mean & 358.19 & mean & 8.22 & mean & 9257.81 & mean & 180.96 & & \\
\hline & & median & 1.0 & median & 103 & median & 5 & median & 515 & median & 14326 & & \\
\hline & & $\min$ & 1.0 & $\min$ & 11 & $\min$ & 1 & $\min$ & 11 & $\min$ & & & \\
\hline & & $\max$ & 10.0 & $\max$ & 3901 & $\max$ & 59 & $\max$ & 230159 & $\max$ & 201.58 & & \\
\hline \multirow[t]{10}{*}{ Rosegarden } & $\mathrm{AC}$ & cum. & 180.0 & cum. & 4737711 & cum. & 96280 & cum. & 159747063 & cum. & 129.73 & 0.001059759 & \multirow[t]{10}{*}{1.02} \\
\hline & & mean & 1.0 & mean & 478.3 & mean & 9.7 & mean & 16127.9 & mean & 123.78127 & & \\
\hline & & median & 1.0 & median & 144 & median & 6 & median & 882 & median & 122.08 & & \\
\hline & & $\min$ & 1.0 & $\min$ & 4 & $\min$ & 1 & $\min$ & 4 & $\min$ & -65.14 & & \\
\hline & & $\max$ & 41.0 & $\max$ & 179332 & $\max$ & 2939 & $\max$ & 8739389 & $\max$ & 213.41 & & \\
\hline & TC & cum. & 177.0 & cum. & 54252 & cum. & 1614 & cum. & 3376737 & cum. & 104.52 & 0.140699523 & \\
\hline & & mean & 3.5 & mean & 1085.0 & mean & 27.36 & mean & 67534.7 & mean & 102.48862 & & \\
\hline & & median & 1.0 & median & 561.5 & median & 13 & median & 7029 & median & 99.095 & & \\
\hline & & $\min$ & 1.0 & $\min$ & & $\min$ & 1 & $\min$ & 8 & $\min$ & 0 & & \\
\hline & & $\max$ & 35.0 & $\max$ & 21126 & $\max$ & 143 & $\max$ & 131298137 & $\max$ & 168.81 & & \\
\hline \multirow[t]{10}{*}{ Apache Shiro } & $\mathrm{AC}$ & cum. & 5786.0 & cum. & 565003 & cum. & 25368 & cum. & 8732435 & cum. & 156.34 & 0.198069287 & \multirow[t]{10}{*}{185.21} \\
\hline & & mean & 1.52 & mean & 148.45 & mean & 6.67 & mean & 2294.38 & mean & 129.81599 & & \\
\hline & & median & 1.0 & median & 57 & median & 5 & median & 275 & median & 133.29 & & \\
\hline & & $\min$ & 1.0 & $\min$ & 8 & $\min$ & 1 & $\min$ & 8 & $\min$ & 0 & & \\
\hline & & $\max$ & 34.0 & $\max$ & 6107 & $\max$ & 100 & $\max$ & 431747 & $\max$ & 208.72 & & \\
\hline & TC & cum. & 545.0 & cum. & 131359 & cum. & 3409 & cum. & 1666934 & cum. & 146.58 & 0.106403749 & \\
\hline & & mean & 1.11 & mean & 269.73 & mean & 7 & mean & 3422.86 & mean & 180.30 & & \\
\hline & & median & 1.0 & median & 116 & median & 6 & median & 696 & median & 148.74 & & \\
\hline & & $\min$ & 1.0 & $\min$ & 8 & $\min$ & 1 & $\min$ & & $\min$ & 0 & & \\
\hline & & $\max$ & 7.0 & $\max$ & 3341 & $\max$ & 33 & $\max$ & 103571 & $\max$ & 192.26 & & \\
\hline \multirow[t]{5}{*}{ Apache Sqoop } & $\mathrm{AC}$ & cum. & 12334.0 & cum. & 2006332 & cum. & 52696 & cum. & 35816170 & cum. & 137.97 & 0.147523533 & \multirow[t]{5}{*}{2.45} \\
\hline & & mean & 1.9 & mean & 312.3 & mean & 8.2 & mean & 5574.5 & mean & 126.42625 & & \\
\hline & & median & 1.0 & median & 142 & median & 6 & median & 912 & median & 129.26 & & \\
\hline & & $\min$ & 1.0 & $\min$ & & $\min$ & 1 & $\min$ & 4 & $\min$ & 0 & & \\
\hline & & $\max$ & 37.0 & $\max$ & 44730 & $\max$ & 1713 & $\max$ & 1079676 & $\max$ & 204.57 & & \\
\hline
\end{tabular}




\begin{tabular}{|c|c|c|c|c|c|c|c|c|c|c|c|c|c|}
\hline \multirow[t]{6}{*}{ Application } & \multirow{6}{*}{$\mathrm{TC}$} & \multicolumn{2}{|c|}{ V(G) } & \multicolumn{2}{|c|}{ V } & \multicolumn{2}{|c|}{ D } & \multicolumn{2}{|c|}{$\mathbf{E}$} & \multicolumn{2}{|c|}{ MI } & \multirow{6}{*}{\begin{tabular}{|c|} 
CC/LOC \\
0.122440045
\end{tabular}} & \multirow[t]{6}{*}{$\mathrm{V}(\mathrm{G}) \mathrm{AC} / \mathrm{TC}$} \\
\hline & & cum. & 5034.0 & cum. & 998984 & cum. & 25721 & cum. & 15276608 & cum. & 137.2 & & \\
\hline & & mean & 1.50 & mean & 296.3 & mean & 7.6 & mean & 4531.8 & mean & 120.04 & & \\
\hline & & median & 1.0 & median & 133 & median & 6 & median & 798 & median & 100.17 & & \\
\hline & & $\min$ & 1.0 & $\min$ & & $\min$ & 1 & $\min$ & & $\min$ & & & \\
\hline & & $\max$ & 17.0 & $\max$ & 12361 & $\max$ & 52 & $\max$ & 494440 & $\max$ & 186.23 & & \\
\hline \multirow[t]{10}{*}{ SQuirrel SQL } & \multirow[t]{5}{*}{$\mathrm{AC}$} & cum. & 27870.0 & cum. & 3881922 & cum. & 113672 & cum. & 69856735 & cum. & 143.81 & \multirow[t]{5}{*}{0.143022092} & \multirow[t]{10}{*}{7.42} \\
\hline & & mean & 1.7 & mean & 195.51 & mean & 5.73 & mean & 3518.34 & mean & 132.4384 & & \\
\hline & & median & 1.0 & median & 62 & median & 4 & median & 264 & median & 130.805 & & \\
\hline & & $\min$ & 1.0 & $\min$ & 8 & $\min$ & 1 & $\min$ & 8 & $\min$ & 0 & & \\
\hline & & $\max$ & 176.0 & $\max$ & 17455 & $\max$ & 112 & $\max$ & 1532944 & $\max$ & 208.48 & & \\
\hline & \multirow[t]{5}{*}{ TC } & cum. & 3757.0 & cum. & 528544 & cum. & 17843 & cum. & 7415573 & cum. & 152.2 & \multirow[t]{5}{*}{0.135328867} & \\
\hline & & mean & 1.2 & mean & 142.73 & mean & 4.82 & mean & 2002.59 & mean & 131.16212 & & \\
\hline & & median & 1.0 & median & 82 & median & 5 & median & 336 & median & $\begin{array}{r}126.52 \\
\end{array}$ & & \\
\hline & & $\min$ & 1.0 & $\min$ & 4 & $\min$ & 1 & $\min$ & 4 & $\min$ & & & \\
\hline & & $\max$ & 15.0 & $\max$ & 7114 & $\max$ & 53 & $\max$ & 263218 & $\max$ & 184.96 & & \\
\hline \multirow[t]{10}{*}{ VLC } & \multirow[t]{5}{*}{$\mathrm{AC}$} & cum. & 83145.0 & cum. & 13126768 & cum. & 268177 & cum. & 502490798 & cum. & 117.63 & \multirow[t]{5}{*}{0.175945539} & \multirow[t]{10}{*}{108.40} \\
\hline & & mean & 4.3.0 & mean & 674.1 & mean & 13.8 & mean & 25805.8 & mean & 106.25235 & & \\
\hline & & median & 1.0 & median & 334 & median & 12 & median & 3938 & median & 106.44 & & \\
\hline & & $\min$ & 1.0 & $\min$ & & $\min$ & 1 & $\min$ & & $\min$ & -20.85 & & \\
\hline & & $\max$ & 326.0 & $\max$ & 141295 & $\max$ & 3002 & $\max$ & 8783253 & $\max$ & 208.29 & & \\
\hline & \multirow[t]{5}{*}{$\mathrm{TC}$} & cum. & 767.0 & cum. & 153540 & cum. & 3258 & cum. & 5624244 & cum. & 112.08 & \multirow[t]{5}{*}{0.154918198} & \\
\hline & & mean & 4.0 .0 & mean & 791.4 & mean & 16.8 & mean & 28990.9 & mean & 100.32777 & & \\
\hline & & median & 1.0 & median & 443 & median & 14 & median & 5797 & median & 103.27 & & \\
\hline & & $\min$ & 1.0 & $\min$ & 34 & $\min$ & 3 & $\min$ & 102 & $\min$ & & & \\
\hline & & $\max$ & 156.0 & $\max$ & 15344 & $\max$ & 390 & $\max$ & 902943 & $\max$ & 160.08 & & \\
\hline Voldemort & $\mathrm{AC}$ & cum. & 24733.0 & cum. & 3716241 & cum. & 100984 & cum. & 73526714 & cum. & 137.84 & 0.180837763 & 4.49 \\
\hline & & mean & 1.9 & mean & 249.78 & mean & 6.79 & mean & 4941.98 & mean & 124.29038 & & \\
\hline & & median & 1.0 & median & 106 & median & 5 & median & 590 & median & 128.58 & & \\
\hline & & $\min$ & 1.0 & $\min$ & & $\min$ & 1 & $\min$ & & $\min$ & 0 & & \\
\hline & & $\max$ & 92.0 & $\max$ & 57976 & $\max$ & 218 & $\max$ & 2630968 & $\max$ & 206.74 & & \\
\hline & TC & cum. & 5508.0 & cum. & 1583546 & cum. & 30872 & cum. & 40745773 & cum. & 119.15 & 0.108346283 & \\
\hline & & mean & 2.1 & mean & 500.97 & mean & 9.774438469 & mean & 12890.1528 & mean & 114.71663 & & \\
\hline & & median & 1.0 & median & 245 & median & 9 & median & 2266.5 & median & 114.85 & & \\
\hline & & $\min$ & 1.0 & $\min$ & & $\min$ & 1 & $\min$ & & $\min$ & 0 & & \\
\hline & & $\max$ & 20.0 & $\max$ & 14033 & $\max$ & 124 & $\max$ & 1740092 & $\max$ & 198.13 & & \\
\hline Vscode & $\mathrm{AC}$ & cum. & 3992.0 & cum. & 1505599 & cum. & 22997 & cum. & 64239046 & cum. & 130.99 & 0.103203123 & 12.79 \\
\hline & & mean & 2.2 .0 & mean & 428.95 & mean & 6.551851852 & mean & 18301.72251 & mean & 134.33769 & & \\
\hline & & median & 1.0 & median & 180 & median & 8 & median & 1460 & median & 142.8 & & \\
\hline & & $\min$ & 1.0 & $\min$ & & $\min$ & 1 & $\min$ & & $\min$ & & & \\
\hline & & $\max$ & 48.0 & $\max$ & 198761 & $\max$ & 157 & $\max$ & 10203587 & $\max$ & 160.98 & & \\
\hline
\end{tabular}




\begin{tabular}{|c|c|c|c|c|c|c|c|c|c|c|c|c|c|}
\hline \multirow[t]{6}{*}{ Application } & \multirow{6}{*}{ TC } & \multicolumn{2}{|c|}{$\mathbf{V}(\mathbf{G})$} & \multicolumn{2}{|c|}{ V } & \multicolumn{2}{|c|}{ D } & \multicolumn{2}{|c|}{$\mathbf{E}$} & \multicolumn{2}{|c|}{ MI } & \multirow{6}{*}{\begin{tabular}{|c|} 
CC/LOC \\
0.29489603
\end{tabular}} & \multirow[t]{6}{*}{$\mathrm{V}(\mathrm{G}) \mathrm{AC} / \mathrm{TC}$} \\
\hline & & cum. & 312.0 & cum. & 204511 & cum. & 4698 & cum. & 4559041 & cum. & 200.04 & & \\
\hline & & mean & 2.6 & mean & 30.9239482 & mean & 7.601941748 & mean & 7377.088997 & mean & 214.07 & & \\
\hline & & median & 1.0 & median & 146.5 & median & 6 & median & 972 & median & 198.56 & & \\
\hline & & $\min$ & 1.0 & $\min$ & & $\min$ & 1 & $\min$ & 4 & $\min$ & & & \\
\hline & & $\max$ & 11.0 & $\max$ & 13480 & $\max$ & 88 & $\max$ & 1186240 & $\max$ & 218.71 & & \\
\hline \multirow[t]{10}{*}{ WorldWind } & $\mathrm{AC}$ & cum. & 57172.0 & cum. & 9188614 & cum. & 253434 & cum. & 254162257 & cum. & 135.24 & 0.173573541 & \multirow[t]{10}{*}{57.98} \\
\hline & & mean & 2.2 & mean & 353.0 & mean & 9.74 & mean & 966.66 & mean & 107.14501 & & \\
\hline & & median & 1.0 & median & 125 & median & 7 & median & 854 & median & 107.73 & & \\
\hline & & $\min$ & 1.0 & $\min$ & 4 & $\min$ & 1 & $\min$ & 4 & $\min$ & 0 & & \\
\hline & & $\max$ & 117.0 & $\max$ & 260991 & $\max$ & 6021 & $\max$ & 23108544 & $\max$ & 171.85 & & \\
\hline & $\mathrm{TC}$ & cum. & $\begin{array}{l}986.0 \\
\end{array}$ & cum. & 373735 & cum. & 6450.00 & cum. & 6397181.00 & cum. & 118.21 & 0.064958166 & \\
\hline & & mean & 1.6 & mean & 606.75 & mean & 19.47.00 & mean & 10385.03 .00 & mean & 123.60688 & & \\
\hline & & median & 1.0 & median & 307.50 & median & 9.00 & median & 2710.00 & median & 126.69 & & \\
\hline & & $\min$ & 1.0 & $\min$ & 11.00 & $\min$ & 2.00 & $\min$ & 22.00 & $\min$ & & & \\
\hline & & $\max$ & 23.0 & $\max$ & 18043.00 & $\max$ & 50.00 & $\max$ & 721720.00 & $\max$ & 210.18 & & \\
\hline
\end{tabular}


Table 4. MAXIMUM CYCLOMATIC COMPLEXITY AND MEDIAN VALUES, RESULTS FOR WILCOXON'S RANK SUM TEST, AND KOLMOGOROV SMIRNOV TEST

\begin{tabular}{|c|c|c|c|c|c|c|c|c|}
\hline \multirow[t]{2}{*}{ Application } & \multicolumn{2}{|c|}{ Maximum V(G) values } & \multicolumn{2}{|c|}{ Median V(G) values } & \multirow{2}{*}{$\begin{array}{r}\text { Wilcoxon RS } \\
\text { p-value }\end{array}$} & \multicolumn{2}{|c|}{ Kolmogorov Smirnov Test } & \multirow{2}{*}{$\begin{array}{r}\text { T-test } \\
\text { p-value }\end{array}$} \\
\hline & $\mathbf{A C}$ & $\mathbf{T C}$ & $\mathbf{A C}$ & $\mathbf{T C}$ & & D value & p-value & \\
\hline Amorok & 47 & 14 & 1 & 1 & $2.20 \mathrm{E}-16$ & 0.26754 & $2.20 \mathrm{e}-16$ & $2.2 \mathrm{e}-16$ \\
\hline Amule & 219 & 55 & 1 & 1 & $3.01 \mathrm{E}-15$ & 0.18496 & $2.68 \mathrm{e}-13$ & $9.323 e-08$ \\
\hline Apache Ant & 82 & 21 & 1 & 1 & $<2.2 \mathrm{e}-16$ & 0.34917 & $2.20 \mathrm{e}-16$ & $2.2 \mathrm{e}-16$ \\
\hline Apache httpd & 350 & 25 & 2 & 2.5 & 0.4903 & 0.18012 & 0.3479 & 0.4997 \\
\hline Audacity & 299 & 3 & 2 & 1 & 0.01929 & 0.28041 & 0.1502 & $4.808 \mathrm{e}-10$ \\
\hline Bitcoin & 356 & 45 & 1 & 1 & $<2.2 \mathrm{e}-16$ & 0.39585 & $2.20 \mathrm{e}-16$ & $2.2 \mathrm{e}-16$ \\
\hline Client & 650 & 15 & 1 & 1 & $2.20 \mathrm{E}-16$ & 0.44689 & $2.20 \mathrm{e}-16$ & $2.2 \mathrm{e}-16$ \\
\hline Curl & 724 & 97 & 3 & 2 & $<2.2 \mathrm{e}-16$ & 0.38092 & $2.20 \mathrm{e}-16$ & 0.02849 \\
\hline Ditaa & 98 & 7 & 1 & 1 & 0.1441 & 0.13571 & 0.6692 & 0.003986 \\
\hline Django & 49 & 21 & 1 & 1 & $2.20 \mathrm{E}-16$ & 0.3104 & $2.20 \mathrm{e}-16$ & $2.2 \mathrm{e}-16$ \\
\hline Filezilla & 275 & 3 & 2 & 1 & $4.677 \mathrm{e}-06$ & 0.44386 & $4.828 \mathrm{e}-05$ & $2.2 \mathrm{e}-16$ \\
\hline FoundationDB & 610 & 72 & 1 & 2 & 0.0005206 & 0.24529 & 0.0002236 & 0.5082 \\
\hline Handbrake & 136 & 196 & 1 & 1 & 0.6492 & 0.0032144 & 0.97 & 0.7124 \\
\hline Hibernate & 29 & 7 & 1 & 1 & $2.20 \mathrm{E}-16$ & 0.27825 & $2.20 \mathrm{e}-16$ & $2.2 \mathrm{e}-16$ \\
\hline Hibernate & 69 & 20 & 1 & 1 & $<2.2 \mathrm{e}-16$ & 0.17297 & $2.20 \mathrm{e}-16$ & $2.2 \mathrm{e}-16$ \\
\hline Industry 1 & 166 & 56 & 3.5 & 2 & $<2.2 \mathrm{e}-16$ & 0.26228 & $2.20 \mathrm{e}-16$ & $2.20 \mathrm{e}-16$ \\
\hline Industry 2 & 144 & 39 & 4 & 3 & 0.0359 & 0.25845 & 0.01052 & 0.1872 \\
\hline Industry 3 & 125 & 25 & 5 & 2 & $1.52 \mathrm{e}-10$ & 0.38449 & $7.46 \mathrm{e}-07$ & $2.20 \mathrm{e}-16$ \\
\hline Industry 4 & 15 & 4 & 1 & 1 & 0.07585 & 0.9506 & 0.9506 & 0.02329 \\
\hline Industry 5 & 11 & 3 & 1 & 1 & 0.8373 & 0.19513 & 0.955 & 0.9989 \\
\hline Inkscape & 262 & 5 & 1 & 1 & $2.2 \mathrm{e}-16$ & 0.13856 & 0.03036 & $2.2 \mathrm{e}-16$ \\
\hline Jakarta jmeter & 51 & 15 & 1 & 1 & $2.89 \mathrm{e}-16$ & 0.27517 & $2.20 \mathrm{e}-16$ & $2.331 \mathrm{e}-11$ \\
\hline Jfreechart & 51 & 21 & 1 & 1 & $<2.2 \mathrm{e}-16$ & 0.22655 & $2.20 \mathrm{e}-16$ & 0.7991 \\
\hline JRuby & 426 & 9 & 1 & 1 & $5.94 \mathrm{e}-09$ & 0.1369 & $5.04 \mathrm{e}-06$ & $2.2 \mathrm{e}-16$ \\
\hline Keepassx & 48 & 11 & 1 & 1 & $5.62 \mathrm{e}-15$ & 0.26862 & $1.99 \mathrm{e}-10$ & $8.553 e-16$ \\
\hline lightGBM & 35 & 6 & 2 & 1 & $8.88 \mathrm{E}-08$ & 0.27856 & $3.89 \mathrm{e}-06$ & $2.2 \mathrm{e}-16$ \\
\hline Mongo & 10019 & 83 & 1 & 1 & $2.20 \mathrm{e}-16$ & 0.10573 & $2.20 \mathrm{e}-16$ & $2.2 \mathrm{e}-16$ \\
\hline MPV Player & 107 & 2 & 3 & 1 & $1.728 \mathrm{e}-09$ & 0.67718 & $6.099 \mathrm{e}-10$ & $2.2 \mathrm{e}-16$ \\
\hline Musescore & 243 & 13 & 1 & 1 & $2.20 \mathrm{E}-16$ & 0.64631 & $2.20 \mathrm{e}-16$ & $2.2 \mathrm{e}-16$ \\
\hline Mypaint & 54 & 21 & 1 & 1 & 0.1563 & 0.084361 & 0.4691 & 0.2201 \\
\hline Netdata & 231 & 41 & 3 & 1 & $2.20 \mathrm{E}-16$ & 0.5858 & $2.20 \mathrm{e}-16$ & $2.2 \mathrm{e}-16$ \\
\hline NMap & 594 & 31 & 1 & 2 & 0.07921 & 0.98478 & 0.2868 & 0.76 \\
\hline Notepad ++ & 734 & 2097 & 1 & 1 & $2.20 \mathrm{E}-16$ & 0.3032 & $2.20 \mathrm{e}-16$ & $2.2 \mathrm{e}-16$ \\
\hline Onion & 38 & 69 & 2 & 6 & $2.20 \mathrm{E}-16$ & 0.43752 & $2.20 \mathrm{e}-16$ & $2.2 \mathrm{e}-16$ \\
\hline Opn XML SDK & 245 & 23 & 1 & 1 & $2.20 \mathrm{E}-16$ & 0.056495 & $5.26 \mathrm{e}-07$ & $2.2 \mathrm{e}-16$ \\
\hline
\end{tabular}




\begin{tabular}{|l|r|r|r|r|r|r|r|r|}
\hline Application & Maximum V(G) values & Median V(G) values & Wilcoxon RS & \multicolumn{2}{|r|}{ Kolmogorov Smirnov Test } & T-test \\
\hline & $\mathbf{A C}$ & $\mathbf{T C}$ & $\mathbf{A C}$ & $\mathbf{T C}$ & $\mathbf{p}$-value & D value & p-value & p-value \\
\hline PDF Creator & 38 & 28 & 1 & 1 & $2.2 \mathrm{e}-16$ & 0.10232 & $2.2 \mathrm{e}-16$ & $2.2 \mathrm{e}-16$ \\
\hline Powershell & 129 & 10 & 1 & 1 & $1.90 \mathrm{E}-07$ & 0.13465 & 0.0006311 & $6.402 \mathrm{e}-12$ \\
\hline Rosegarden & 41 & 35 & 1 & 1 & 0.02563 & 0.17542 & 0.0936 & 0.6563 \\
\hline Apache Shiro & 34 & 2 & 1 & 1 & $4.367 \mathrm{e}-12$ & 0.075419 & $8.999 \mathrm{e}-09$ & $1.196 \mathrm{e}-06$ \\
\hline Apache Sqoop & 37 & 17 & 1 & 1 & $5.91 \mathrm{e}-16$ & 0.081698 & $2.41 \mathrm{e}-13$ & $6.8 \mathrm{e}-16$ \\
\hline SQuirrel SQL & 176 & 15 & 1 & 1 & $<2.2 \mathrm{e}-16$ & 0.29846 & $2.20 \mathrm{e}-16$ & $2.2 \mathrm{e}-16$ \\
\hline VLC & 326 & 156 & 1 & 1 & 0.0005712 & 0.13281 & 0.002055 & 0.06574 \\
\hline Voldemort & 92 & 20 & 1 & 1 & $5.81 \mathrm{E}-09$ & 0.14672 & $2.20 \mathrm{e}-16$ & 0.0005795 \\
\hline Vscode & 48 & 11 & 1 & 1 & $2.2 \mathrm{e}-16$ & 0.31511 & $2.2 \mathrm{e}-16$ & $2.2 \mathrm{e}-16$ \\
\hline WorldWind & 117 & 23 & 1 & 1 & $2.2 \mathrm{e}-16$ & 0.01596 & $2.20 \mathrm{e}-16$ & $2.2 \mathrm{e}-16$ \\
\hline & & & & & & & & \\
\hline
\end{tabular}

\subsection{Inferential Statistics}

Previous sections confirm our intuition that $\mathrm{TC}$ tends to be simpler than $\mathrm{AC}$ (Introduction) but that frequency distributions of complexity values are not normal, heavily skewed and that therefore we cannot overly rely on descriptive statistics (Chapter 3), thereby calling for the use of non-parametric statistical methods.

The mean values are highly affected by the distribution as for almost all objects, there are a higher number of data point with a cyclomatic complexity of 1 which skews the distribution to the left thereby affecting the mean and median. This is very predominant in application code. The test code rather has less skewness in the distribution and less amount of data points with a cyclomatic complexity less than 5 as shown in the box plot. Also, looking at the boxplots and sample distributions in Appendix A, there are way many more AC methods with a cyclomatic complexity value higher than 10 (it goes up to 10019 Table 4), than for TC.

First, we proceeded to confirm our intuition by performing a Shapiro-Wilk Normality test on the datasets to check whether the normality assumption of the t-test is satisfied. The results in Appendix D (Table 7) reject the normality assumption for all our distributions. It is therefore, as expected, not appropriate to use parametric tests. Also, another possible reason why all objects reject the null hypothesis of the Shapiro-Wilk is that when the dataset has more than 5000 (as in the case of Shapiro-Wilk in R), the slightest deviation from normality will lead to a rejection of the null hypothesis. For a large dataset, 
even big departures from normality will not easily be detected. In such a case, graphical representations like histogram, QQ plots and boxplots are enough to judge the deviations from normality. Though in such instances, graphical representation of skewness, kurtosis and outliers suffice to predict non-normality of the data, performing the test will give more accurate and valid results especially on an approximately normal data. Recall from Chapter 3 that we select non-parametric tests for non-normal data. In this analysis, we will focus on using Wilcoxon Rank Sum test as our primary test and use Kolmogorov Smirnov as a confirmatory test. We select Wilcoxon Rank Sum Test over Kolmogorov Smirnov due to its high power as explained in Chapter 3. With these tests we compare the distribution of AC method cyclomatic values and the TC method cyclomaic values for each object separately.

In recording the mean values, we encounter situations where the average values of two variables look almost similar. In such an instance, it is possible to assume that these values are almost the same. We need to verify that these values are either statistically different or almost similar. Therefore, for instances where the $\mathrm{p}$-value is greater than the alpha significant level, we can reject the difference in the mean values. Table 4 show statistical test results when we use the Wilcoxon Rank Sum Test and the Kolmogorov Smirnov Test. All p-values for Wilcoxon Rank Sum test are much smaller than the $0.05 \alpha$ level, with the exception of Apache httpd (0.49), Ditaa (0.06), Handbrake (0.64), Mypaint (0.15), Shiro (0.06) and VSCode (0.54).

In Section 5.1.2, we observed instances of objects where TC shows higher average cyclomatic complexity values than AC. We will proceed to verify with Wilcoxon Rank Sum Test if, perhaps, these values are drawn from the same distribution or are statistically different by which we can either accept the values or reject them as outliers. For objects such as Apache, Mypaint and VSCcode, which previously had their average TC method cyclomatic complexity value greater than AC values, results of Wilcoxon Rank Sum Test (p-values) lead us to reject the null hypothesis. At this point we consider these objects as outliers, which allow us to reject the null hypothesis of our tests for all case study systems but these: Kolmogorov Smirnov Test also reports a similar set of values that lead to the rejection of the null hypothesis: Apache httpd (p-value of 0.34), Ditaa (p-value of 0.67), Handbrake (p-value of 0.9), VSCode (p-value of 0.18), Mypaint (p-value of 0.46), and Shiro 
(p-value of 0.45), Rosegarden (p-value of 0.09). Recall that the variation in the values between results of Kolmogorov Smirnov and Wilcoxon Rank Sum is because Wilcoxon Rank Sum uses ranks to calculate the p-value and therefore it is sensitive to a shift in the median values while Kolmogorov Smirnov calculates the p-value using a shift in the probability distribution frequency. Recall that the null and alternate hypotheses for Wilcoxon Rank Sum are:

- Ho: There is a statistically significant difference between the median method cyclomatic complexity values of $\mathrm{AC}$ and $\mathrm{TC}$ for each objects.

- $\mathrm{H}_{1}$ : The distributions of $\mathrm{AC}$ and TC method cyclomatic complexity values are drawn from different distributions.

Results, as discussed previously, allow us to reject the null hypothesis for the majority of the objects, which shows that, as similar as the cyclomatic complexity mean and median values for $\mathrm{AC}$ and $\mathrm{TC}$ methods may seem (Table 3), they are statistically significantly different.

As discussed earlier there are some instances for which we have higher mean values for TC than AC.We therefore regard these objects to have more complex test code methods than application code. The ratio for AC to TC are given as: Amorok (1.1:1.2), Light GBM (1.0:1.6), Mongo (1.1:2.1), Notepad ++ (5.1:14.3), Open XML (1.4:1.8), Onion (2.9:10.7), PDF Creator (1.45:1.48), Rosegarden (0.1:3.5) and Voldemort (1.9:2.6).

Though some instance have higher mean values for TC than AC contrary to what we observe for all the other case study systems, however, for some instances, the statistical test results does not allow us to reject the null hypothesis. Mean values for object like Apache Httpd (5.0:5.7), Foundation (3.1:4.0), Mypaint (2.5:2.6), VSCode (2.2:2.6) which tend to be greater than production code will be regarded as outliers. Likewise, that of Ditaa, Handbrake, Shiro and Rosegarden which tend to have higher average values for AC than for TC will also be regarded as outliers due to the rejection of the null hypothesis. (Boxplots in Appendix A ).

To conclude, all the metrics we used show AC methods tend to have a higher complexity than TC methods: The cyclomatic complexity values of AC methods are higher than TC method complexity values; Their Halstead values are higher; the mean and median values of AC complexity are higher than TC complexity values. And for a slight difference 
in the mean values, statistical tests show that the difference is very statistically significant and cannot be ignored. Test code as hypothesized by some authors is expected to be simple but the results gained so far proves otherwise.

\subsection{On the Use of Non-Parametric Statistical Tests}

In this study, we have privileged the use of non-parametric statistical tests over parametric statistical tests. We believe it is important that, in addition to justifying the use of non-parametric tests by the fact distributions are not normal, we go further and explain why non-parametric statistical tests are better suited in our study.

In our attempt to use statistical tests to explain the difference between $\mathrm{AC}$ complexity and TC complexity, one may argue that it suffices to use the student's t-test even when the normality assumption is rejected, given that the sample of method complexity values is large (recall the NOM column in Table 2). With such large samples, the Central Limit Theorem (CLT) suggests that the use of a t-test is legitimate [70]. Although a t-test may legitimately be used in our context, since we have large samples, we argue it is inappropriate to use it in our context on the following grounds. (1) In our results, the population size, that is the set of methods, is fixed for each object and does not allow for an increase in the sample size which would have, in some sense, warranted the use of parametric tests given it would have been possible to increase the sample size (as parametric tests are powerful in such cases). (2) Our analysis does not warrant the use of parametric tests for a non-normal distribution because it rejects one of the main assumptions of the ttest which is the normality and homogeneity of variance (homoscedasticity) assumptions as explained in Section 3.4. A test for normality rejects the normality assumption which makes it erroneous to use a t-test, leading to a potential of Type I (false positives) error when using the t-test. (3) In our context, non-parametric tests will perform better than parametric tests (including a t-test) as the former will allow us to detect smaller statistical differences than the latter. (And, indeed, descriptive statistics indicate that mean and median values of AC and TC are close to each other). One may argue that for large sample sizes, the t-test is robust against deviations from the normality and equal variance (homogeneity of variance) assumptions when using the central limit theorem and the probability of large numbers with sample averages. This is only possible for larger dataset out of which a smaller sample is drawn from. The idea is that increasing the sample size would move the distribution towards 
a normal distribution; in our context, we argue, given the heavily skewed distributions we observe, that increasing the set of methods in an object, if that were at all possible from an engineering point of view, would certainly not guarantee we converge towards a normal distribution.

Moreover, CLT (the law of large numbers) does not make a large-sample nonnormal distribution approximately normal to the extent a t-test can be used; rather, only the sampling averages become approximately normally distributed. Therefore, if we desire to test for the difference between the original sample groups, it is not appropriate to compare the differences between some sample averages of the original data. That being said, if it were legitimate to rely on CLT and proceed with a t-test, that would mean that given any non-normal distribution, we can make it normal by taking a larger sample of the data; this is not correct. In this case the sampling average is not relevant. The use of sample averages (through a t-test) rather than original averages will result in different data sets which is not what we would want to compare. One of the assumptions of the t-test is that each population under comparison (not the sample average) should have a normal distribution.

Our analysis favours the use of non-parametric tests due to the skewness of the distributions and the violations to the normality assumption. While we agree, to some extent, with someone who may advocate for the use of the t-test, we conclude that it is not appropriate and it is misleading (Type II error) as one is likely to accept the null hypothesis in certain instance while one will reject the null hypothesis using non-parametric tests like the Wilcoxon Rank Sum test and the Kolmogorov Smirnov test.

The values of Table 4 show significant differences between the results obtained using the parametric t-test and non-parametric tests like Wilcoxon Rank Sum and Kolmogorov Smirnov. In the case of Curl, we observe a p-value of 0.02 (almost at the significance level) using the t-test while the non-parametric tests yield the lowest p-value possible (2.2e-16). Audacity, FileZilla, Handbrake, Inkscape, Keepassx, MPV Player, Open $\mathrm{XML}$ are examples of such high variations in values leading to (potential) error.

A similar observation is made in the cases of Voldemort, Apache Ant, SQuirel SQL, Jakarta, Apache Httpd and Hibernate leading to false positives. In situations where slight differences in values matter, the t-test would not lead to a more convincing conclusion and this, we believe, could be misleading. 
We therefore advocate for the use of non-parametric tests in this study. This is because non-parametric tests perform better on non-normal distributions and can detect changes and shifts in the distributions and median values that the t-test cannot detect. One could argue that the t-test is very robust to violations to the normality and equal variance assumptions and therefore, the t-test would still give satisfactory results for data which are either non-normal or heteroscedastic or both. However, in such circumstances, nonparametric tests would yield superior performance, especially in certain cases where the pvalues are close to the desired significance level.

Results for Heteroscedastic t-test are included in Table 4, along with the results for the Wilcoxon Rank Sum and Kolmogorov-Smirnov tests. As expected, although the t-test may still give acceptable results, it cannot detect some differences which the Wilcoxon Rank Sum and the Kolmogorov detect, and therefore using a t-test we believe can lead to misleading conclusions. This is a confirmation of our initial intuition and the reason for selecting non-parametric statistical tests.

\subsection{On Correlation among Software Metric}

In this section we look at how different kinds of code determine the correlation that exist between software metrics. We will use Spearman's correlation when attempting to confirm whether there is a monotonic relationship between two groups of data. In Table 5, we list the values obtained for Spearman's correlation on AC, comparing Cylomatic Complexity and LOC. In our investigation, almost all the Spearman's rho values obtained for AC imply a high correlation ranging from 0.35 to 0.87 . This results confirms empirical results obtained by a large majority of authors as explained in Chapter 2. However, this does not apply to TC as the results are the opposite, to the point that results indicate a negative correlation for a number of objects. FileZilla has the highest correlation between Cyclomatic Complexity and LOC for AC (0.89) but has one of the least correlation for its TC (0.20). The only application that appears to have highly correlated LOC and CC for both AC and TC is Curl (0.88 and 0.87, respectively) and Foundation DB ( 0.73 and 0.84 respectively). Except for these two, all objects have a high correlation for $\mathrm{AC}$ and a weak correlation for TC.

The empirical results we observed from the related works in Chapter 2 seems to be missing an element in their analysis which is a fundamental part of our study. That is, the 
kind of code affects the correlation of the complexity metrics (the kind of metrics). Therefore, it is not ideal for two complexity metrics to be substituted one for another by the mere fact that some studies suggest a strong correlation between certain metrics (complexity metrics in our case). All these metrics have different objectives and in their attempt to determine the complexity of a piece of code and it is quite erroneous to discredit the explanatory power of one to exist.

For example, we record instances where there is negative correlation - that is as LOC increase, Cyclomatic Complexity decreases: VSCode (-022), Rosgarden (-017), PDF Creator $(-0.22)$, Notepad $++(-0.11)$ and Musescore (-0.49). Musecore has the highest difference with a 0.84 value for $\mathrm{AC}$ and -0.49 for TC. The notion of a strong correlation between LOC and CC cannot be extended to every kind of code. Therefore an attempt to substitute one metric for another, without considering any of these factors (kind of code, kind of software, and kind of metric) will fail. Open XML presents the least form of monotonicity between LOC and CC with values of 0.372 for $\mathrm{AC}$ and 0.07 for TC. It is followed by VSCode which reports 0.55 correlation for AC and 0.22 for TC. Handbrake on the other hand has a low correlation of 0.32 for $\mathrm{AC}$ but has a high correlation for TC. This is contrary to what is observed for most objects (where there is a higher correlation in $\mathrm{AC}$ but a lower correlation for TC).

Though our analysis suggests a high correlation for AC and a very low correlation for TC, it is not right for one to merit the substitute one metric with over another without considering the kind of code, kind of metric and kind of software. And if a one of the metrics is preferred in certain situations, a very strong justifiable reason has to be given for its choice.

In all these instances, we record a p-value of $-2.2 \mathrm{e}-16$ or less for $\mathrm{AC}$ which is strong enough to reject the null hypothesis that there is no correlation. Test code on the other hand has objects where the values of the correlation coefficient can be rejected for $\mathrm{p}$-values greater than the alpha level of significance. The exceptions are Filezilla with a rho value of 0.20 and a p-value of 0.31, MPV Player with a rho value of 0.36 and 0.07 for p-value, Keepassx with a rho value of 0.11 and a p-value of 0.12 , Powershell with 0.01 and 0.78 repectively, Rosegarden with 0.17 and 0.19 , and OpenXML with 0.07 and 0.67. P-values 
for these are higher than the alpha significance level which leads us to accept the null for these cases.

We proceed to repeat the same correlation experiment for the test code written by these same companies and organisation. We assume the same coding principles, methodologies and paradigms are followed in the two kind of code. Our experiment focuses only on the correlation of LOC, CC and HE in test codeas we believe numerous researches including our finding support the correlation that exists between the complexity metrics for application code evident in our results. We are, however, interested in finding whether the same exist for different kind of code, which is test code. In comparing what has been hypothesized to the results gained, Lines of Code and Halstead effect are not correlated at all. Table 6, we observe a sharp difference from what is usually seen in AC. We observe the predominance of low and negative correlation values running through almost all cases. The same observation is made for Halstead Effort and Cyclomatic complexity. There is a very low correlation and the idea of substituting one metric for another is not advisable for TC.

The negative values and low correlation values produced for TC predicts that Cyclomatic complexity, Halstead Effort and Lines of Code do not have any sort of relation and it will therefore be inappropriate to substitute one for the other. In other words, the metrics seem to measure different aspects of test code. While we observe a higher level of complexity for most application code (always perceived to be largely complex) we observe a very low or almost no correlation for test code (always perceived to be simple). The results suggest that the level of complexity seems to affect the level of correlation. Possible reasons (particularly for LOC and CC) include:

1. The predominance of control flow statements in application over test code. For the same amount of code (LOC) in application code and test code, application code may have many logical branches and loops that will, as observed in the results, generate a high cyclomatic complexity value. And therefore $\mathrm{CC}$ will proportionally increase with LOC and invariably have a strong correlation for almost every given amount of LOC. Test code on the other hand may use test frameworks (for instance assert() call in a JUnit test) which hide (i.e., it resides in the framework) the control flow for the evaluation of the oracle leading to a low CC for the same amount of LOC. A Java test not using JUnit would have to 
embed the control flow for the oracle in the test itself which would increase control flow, thereby moderately increasing $\mathrm{CC}$, leading to a higher $\mathrm{CC}$ for the same amount of LOC; resulting in a moderate correlation. We will likely observe a low correlation between CC and LOC in test code as the control flow for test code is always quite simple even for a large piece of code. An embedded control flow in the test code (and general control flow statements in the test code) on one hand will cause a proportional increase of $\mathrm{CC}$ with $\mathrm{LOC}$, resulting in a higher correlation. This results in a changing correlation for test code making it difficult to predict any sort of correlation between LOC and CC, if even there is any at all.

2. The kind of tests (e.g., unit testing, integration testing and system testing) also has an impact on the level of correlation of test code. Unit tests may appear more linear (few branches, simple, and straight) than system test which may require complex set-up and therefore complex control flow. This is because the scope for unit test is smaller, while system tests may need longer, larger and more complex setup which would involve control flow. The use of unit tests alone may result in a low correlation while a system test will report a stronger correlation. This is because for the same amount of LOC, the linearity of unit tests leads to fewer control flow branches and therefore a low CC value. Correlation will be low as $\mathrm{CC}$ does not increase for an increase in LOC without additional control flow. A system test on the other hand, due to complex setup it may require, will have many conditions leading to a large amount of control flow statements, hence a larger CC value for the same amount of LOC (a stronger correlation). The kind of test also impacts the level of complexity and correlation. Our analysis does not differentiate different kinds of tests so we cannot confirm these intuitions. 
Table 5. CORRELATION COEFFICIENT, P-VALUES AND STATISTIC FOR COMPARING CYCLOMATIC COMPLEXITY AND LINES OF CODE

\begin{tabular}{|c|c|c|c|c|c|c|}
\hline \multirow{2}{*}{ Application } & \multicolumn{2}{|c|}{ Spearman's rho } & \multicolumn{2}{|c|}{ Statistic (S) } & \multicolumn{2}{|c|}{ p-value } \\
\hline & Production Code & Test Code & Production Code & Test Code & Production Code & Test Code \\
\hline Amorok & 0.7484398 & 0.2826294 & $9.12 \mathrm{E}+10$ & 119200000 & $<2.2 \mathrm{e}-16$ & $<2.2 \mathrm{e}-16$ \\
\hline Amule & 0.8310898 & 0.6087374 & $5.567 \mathrm{E}+09$ & 6684300 & $<2.2 \mathrm{e}-16$ & $<2.2 \mathrm{e}-16$ \\
\hline Apache Ant & 0.8527345 & 0.313269 & $2.60 \mathrm{E}+10$ & 9340470 & $<2.2 \mathrm{e}-16$ & $<2.2 \mathrm{e}-16$ \\
\hline Apache httpd & 0.811654 & 0.498923 & $2.5 \mathrm{e}+15$ & 8934731 & $<2.2 \mathrm{e}-16$ & $<2.2 \mathrm{e}-16$ \\
\hline Audacity & 0.7731698 & 0.5459371 & $1.40 \mathrm{E}+12$ & 308.76 & $<2.2 \mathrm{e}-16$ & 0.02869 \\
\hline Bitcoin & 0.7745475 & 0.386350 & $1.60 \mathrm{E}+10$ & 15716 & $<2.2 \mathrm{e}-16$ & 0.00089 \\
\hline Client & 0.8458951 & 0.3737259 & $2.577 \mathrm{E}+09$ & 20790000 & $<2.2 \mathrm{e}-16$ & $<2.2 \mathrm{e}-16$ \\
\hline Curl & 0.8797098 & 0.8676859 & 60994000 & 21986000 & $2.20 \mathrm{E}-16$ & $<2.2 \mathrm{e}-16$ \\
\hline Ditaa & 0.8390775 & 0.3717422 & 5424700 & 2824 & $<2.2 \mathrm{e}-16$ & 0.0431 \\
\hline Django & 0.5384983 & 0.0661646 & $9.91 \mathrm{E}+10$ & $7.38 \mathrm{E}+11$ & $<2.2 \mathrm{e}-16$ & $<2.2 \mathrm{e}-16$ \\
\hline Filezilla & 0.8924248 & 0.2002189 & $1.525 \mathrm{E}+09$ & 2620.1 & $<2.2 \mathrm{e}-16$ & 0.3167 \\
\hline FoundationDB & 0.7314024 & 0.8440161 & $1.45 \mathrm{E}+11$ & 10966 & $<2.2 \mathrm{e}-16$ & $<2.2 \mathrm{e}-16$ \\
\hline Handbrake & 0.3529543 & 0.8485892 & $1.93 \mathrm{E}+10$ & $4.642 \mathrm{E}+09$ & $<2.2 \mathrm{e}-16$ & $<2.2 \mathrm{e}-16$ \\
\hline Hibernate & 0.8390727 & 0.3397333 & 98915000 & 117470000 & $<2.2 \mathrm{e}-16$ & $<2.2 \mathrm{e}-16$ \\
\hline Hibernate & 0.6790383 & 0.2627793 & $1.88 \mathrm{E}+11$ & $6.73 \mathrm{E}+10$ & $<2.2 \mathrm{e}-16$ & $<2.2 \mathrm{e}-16$ \\
\hline Industry 1 & 0.05809031 & 0.007931312 & 9601600 & 303950000 & 0.25 & 0.7815 \\
\hline Industry 2 & 0.9209294 & 0.8240325 & 1282.1 & 570860 & $<2.2 \mathrm{e}-16$ & $2.2 \mathrm{e}-16$ \\
\hline Industry 3 & 0.8795279 & 0.3826746 & 4784.1 & 1808300 & $<2.2 \mathrm{e}-16$ & $1.709 \mathrm{e}-10$ \\
\hline Industry 4 & 0.199784 & -0.350367 & $1.558 \mathrm{e}+09$ & 119590 & $<2.2 \mathrm{e}-16$ & 0.001343 \\
\hline Industry 5 & 0.2723476 & -0.3023716 & 21642000 & 72.933 & $4.95 \mathrm{e}-11$ & 0.5098 \\
\hline Inkscape & 0.764367 & 0.2744665 & $8.34 \mathrm{E}+10$ & 160930 & $<2.2 \mathrm{e}-16$ & 0.003712 \\
\hline Jakarta jmeter & 0.7548558 & 0.4901934 & $1.63 \mathrm{E}+10$ & 49467000 & $<2.2 \mathrm{e}-16$ & $<2.2 \mathrm{e}-16$ \\
\hline Jfreechart & 0.8719291 & 0.574748 & 168360 & $1.838 \mathrm{E}+09$ & $<2.2 \mathrm{e}-16$ & $<2.2 \mathrm{e}-16$ \\
\hline JRuby & 0.7998944 & 0.632227 & $2.23 \mathrm{E}+11$ & 4941700 & $<2.2 \mathrm{e}-16$ & $<2.2 \mathrm{e}-16$ \\
\hline Keepassx & 0.839651 & 0.1165798 & 58040000 & 749180 & $<2.2 \mathrm{e}-16$ & 0.1278 \\
\hline lightGBM & 0.8317869 & 0.4278641 & 78925000 & 64974 & $<2.2 \mathrm{e}-16$ & $3.21 \mathrm{E}-05$ \\
\hline Mongo & 0.6395512 & 0.2862239 & $2.80 \mathrm{E}+14$ & $2.31 \mathrm{E}+11$ & $<2.2 \mathrm{e}-16$ & $<2.2 \mathrm{e}-16$ \\
\hline MPV Player & 0.8607678 & 0.3645325 & $3.324 \mathrm{E}+09$ & 1461.6 & $<2.2 \mathrm{e}-16$ & 0.07989 \\
\hline Musescore & 0.8236439 & -0.4906538 & $3.72 \mathrm{E}+11$ & 303500000 & $<2.2 \mathrm{e}-16$ & $<2.2 \mathrm{e}-16$ \\
\hline Mypaint & 0.6853768 & 0.2129898 & $2.094 \mathrm{E}+09$ & 147530 & $<2.2 \mathrm{e}-16$ & 0.02995 \\
\hline Netdata & 0.8607678 & 0.3653626 & $3.324 \mathrm{E}+09$ & 3151000 & $<2.2 \mathrm{e}-16$ & $3.19 \mathrm{E}-11$ \\
\hline NMap & 0.7815778 & 0.2179744 & $1.26 \mathrm{E}+10$ & 5398800 & $<2.2 \mathrm{e}-16$ & $4.329 \mathrm{e}-05$ \\
\hline Notepad plus plus & 0.8666138 & -0.1102369 & $1.141 \mathrm{E}+09$ & 47379000 & $<2.2 \mathrm{e}-16$ & 0.005421 \\
\hline Onion & 0.8001629 & 0.2103819 & 59004000 & 2677600 & $<2.2 \mathrm{e}-16$ & 0.0004663 \\
\hline Opn XML SDK & 0.3729942 & 0.0076572 & $2.08 \mathrm{E}+13$ & $4.412 \mathrm{E}+09$ & $<2.2 \mathrm{e}-16$ & 0.6757 \\
\hline PDF Creator & 0.587326 & -0.2241322 & $2.74 \mathrm{E}+10$ & $2.23 \mathrm{E}+10$ & $<2.2 \mathrm{e}-16$ & $<2.2 \mathrm{e}-16$ \\
\hline Powershell & 0.8023586 & 0.0167814 & $8.62 \mathrm{E}+11$ & 2947100 & $<2.2 \mathrm{e}-16$ & 0.7869 \\
\hline Rosegarden & 0.8020894 & -0.1701537 & $3.21 \mathrm{E}+10$ & 40043 & $<2.2 \mathrm{e}-16$ & 0.1976 \\
\hline Shiro & 0.5901554 & 0.3046109 & 2706100000 & 13059000 & $<2.2 \mathrm{e}-16$ & $7.898 \mathrm{e}-12$ \\
\hline Sqoop & 0.7271721 & 0.6016462 & $1.25 \mathrm{E}+10$ & 2438400000 & $<2.2 \mathrm{e}-16$ & $2.2 \mathrm{e}-16$ \\
\hline SQuirrel SQL & 0.773741 & 0.4688885 & $1.83 \mathrm{E}+11$ & $2.707 \mathrm{E}+09$ & $<2.2 \mathrm{e}-16$ & $<2.2 \mathrm{e}-16$ \\
\hline VLC & 0.8378148 & 0.1542142 & $2.02 \mathrm{E}+11$ & 2255800 & $<2.2 \mathrm{e}-16$ & 0.01426 \\
\hline Voldemort & 0.7740988 & 0.5696082 & $8.95 \mathrm{E}+10$ & $1.423 \mathrm{E}+09$ & $<2.2 \mathrm{e}-16$ & $<2.2 \mathrm{e}-16$ \\
\hline Vscode & 0.5588973 & -0.2241322 & 920400000 & $2.23 \mathrm{E}+10$ & $<2.2 \mathrm{e}-16$ & $2.20 \mathrm{E}-16$ \\
\hline WorldWind & 0.8589899 & 0.1008642 & $4.18 \mathrm{E}+11$ & 64493000 & $<2.2 \mathrm{e}-16$ & 0.005538 \\
\hline
\end{tabular}


Table 6. CORRELATION OF LOC VS CC AND CC VS HALSTEAD EFFORT FOR

TEST CODE

\begin{tabular}{|l|r|r|r|r|r|}
\hline \multirow{2}{*}{ Test Code } & \multicolumn{2}{|c|}{ Spearman's rho } & \multirow{2}{*}{ Test Code } & Spearman's rho & \\
\cline { 2 - 3 } & LOC vs HE & CC vs HE & & LOC vs HE & CC vs HE \\
\hline Amorok & 0.318546 & -0.4688933 & JRuby & 0.2359847 & 0.8640336 \\
\hline Amule & 0.1334175 & 0.7890728 & Keepassx & 0.2895168 & 0.3582617 \\
\hline Apache Ant & 0.5092279 & 0.3068285 & LightGBM & 0.5526408 & 0.5969304 \\
\hline Apache HTTPD & 0.9457816 & 0.3164342 & Mongo DB & 0.3298503 & 0.4910320 \\
\hline Audacity & 0.5092279 & 0.3574877 & MPV Player & 0.8258287 & 0.4508373 \\
\hline Bitcoin & 0.6076619 & 0.5554357 & Musescore & 0.5291308 & -0.0978287 \\
\hline Client & 0.6357196 & 0.3574877 & Mypaint & 0.4719101 & 0.1519379 \\
\hline Curl & 0.6486515 & 0.6700446 & Netdata & 0.9031964 & 0.7323997 \\
\hline Ditaa & 0.2785671 & 0.697921 & NMap & 0.8468941 & 0.4294187 \\
\hline Django & 0.2878933 & 0.2784571 & Notepad ++ & 0.870604 & 0.4610390 \\
\hline Filezilla & 0.09648793 & 0.1635965 & Onion & 0.6768024 & 0.6990263 \\
\hline FoundationDB & 0.5368896 & 0.8224289 & OpenXML & 0.1259020 & 0.2000405 \\
\hline Handbrake & 0.5599499 & 0.8145304 & PDF Creator & 0.5644849 & 0.4747389 \\
\hline Hibernate ORM & 0.2627885 & 0.7172306 & Powershell & 0.3044482 & 0.4629908 \\
\hline Hibernate & 0.316615 & 0.7064122 & Rosegarden & 0.8362338 & 0.80 \\
\hline Industry 1 & 0.0019411 & 0.0311890 & Apache Sqoop & 0.9455115 & 0.5621386 \\
\hline Industry 2 & 0.8813916 & 0.8198210 & Apache Shiro & 0.9411423 & 0.2543022 \\
\hline Industry 3 & -0.2166831 & 0.3156131 & Squirrel SQL & 0.2950118 & 0.7184319 \\
\hline Industry 4 & 0.4269099 & 0.5280768 & VLC & 0.9098565 & 0.029988 \\
\hline Industry 5 & -0.2594373 & 0.9805807 & Voldemort & 0.4178033 & 0.7781585 \\
\hline Inskspace & 0.5663003 & 0.2752109 & Vscode & -0.08548622 & 0.301735 \\
\hline Jfreecahart & 0.39871 & 0.6555923 & WorldWind Java & 0.3597182 & 0.440394 \\
\hline Jakarta Jmaeter & 0.4809119 & 0.6731702 & & & \\
\hline
\end{tabular}




\section{Chapter 6 THREATS TO VALIDITY}

Threats to validity in software engineering are classified into construct, external, internal, and conclusion threats [42]. Construct validity is about the relation between the theory behind the experiment and the observations that are made. Our theory, or hypothesis, following claims from prominent authors (e.g., [41]), is that test code is less complex than the application code it tests. Since tests are typically implemented as methods [10] we compared the complexity of methods in test code with the complexity of methods in application code. Even though there is a debate about what is a good complexity metric for code, we decided to use the well-known cyclomatic complexity and Halstead metrics to measure method complexity (in test and application code) in addition to the number of lines of code (LOC), though we report on results when only using cyclomatic complexity. We argue that these decisions make sense and should not adversely introduce threats to construct validity in our study.

We want to compare (test) code with (application) code and how they affect complexity metrics using open source software and Industry software. We emphasize here that the concept of open source used in this document refers to source code we collected online from repositories and that, the testing effort of open source software development presented in this document through empirical data is not a characterization of the people that contributed the (test/application) code. We acknowledge that not all test code may have been contributed to the repository by the contributors and this may very well affect the conclusions on the adequacy of testing or how well tested most open source code are. The results do not also characterizes the quality of open source software, if one uses the amount of test code as a surrogate measure of quality. What we refer to as Industry code, on the other hand is code that was provided by Industry partners. We selected metrics that have been reported to be often used to measure source code complexity, at the same, recognizing that there is an ongoing debate about whether some of these metrics correlate or not. We therefore selected LOC, cyclomatic complexity and Halsted measures.

In this study we used a set of criteria for the selection of open source projects. One criterion was the selection of an open source object with more than 10k AC LOC. The definition of what makes a program large is quite ambiguous, undefined and very subjective on an individual and organizational level (contexts) and has not been well defined in 
literature. A number of arbitrary minimum threshold values have been suggested on many reputable websites [22][79] (ranging from 10k, 50k, 100k, etc). While we do agree with all these, we have not found, at the time of writing of this document, literature that discusses what may be considered large enough for a study like ours. We believe that an open source projects with more than 10k LOC is large enough to contain sufficient tests for our study, although by looking at ratio values of application code LOC to test code LOC this does not appear to be a sufficient criterion. Also, the size was considered among many factors; it was not the only selection criterion; and therefore it happened that this criterion was not necessarily satisfied (for Industry 4 and 5) as all the criteria taken together resulted in the selection (out of 500 open source software).

External validity is about whether we can generalize results outside of the scope of the study. Of course, our study should be replicated, expended, especially with case study systems from industry and with other complexity metrics. However, our selection of 40 open-source software led us to select what we believe is a representative set of systems. At least they are well-known and popular; they are not trivial. Although general conclusions should be taken with care, our observations for open source are, we believe, generalizable to some extent.

Internal validity is about the treatments and how sure we can be that they cause the outcome we observe. This relates to our selection of complexity metrics, which we already commented on above. This also relates to our measurement of code complexity. To avoid issues when measuring code, we relied on a commercial software package that is used in industry (Understand). This threat also relates to our selection of objects, which we already commented on. We should add that we selected objects that come with both application code and test code that tests that application code, and for which test code can be easily distinguished from application code (i.e., test code is in separate, identifiable software packages) as this facilitated the use of Understand. Despite our effort to select software for which we can easily distinguish test code from application code, some test code may not have been measured as such and may have contributed to application code data and vice versa; we believe that, given the amount of methods we measured, this should only have a very limited impact on the results. We also wanted systems with both application code and test code because comparing application code from one system to test code from another 
system does not make sense: for instance, different developers, with possibly varying development practices/habits would likely be involved thereby introducing differences in application code and test code complexity values that are not due to the nature (test vs. application) of the code. One threat to validity related to the objects, however, is the possible impact of the effort that has been put into the testing activity for these systems. We identified for instance that twelve case study system (Apache Httpd, Audacity, Foundation, Filezilla, Inkscape, Jruby, MPV Player, Mypaint, Powershell, Rosegarden, Shiro and VLC and VSCode) seemed to have been much less tested than the other case study systems. On the topic of sufficiency of tests, it is worth noting that filtering the amount of test cases presented with the $\mathrm{AC}$ is vital. We do recognize that not all tests may have been committed to the repository for the subjects we analyzed and this may play down on testing effort of the developers. While the testing effort presented in this material is not a characterization of the developers for such subjects, we believe filtering the LOC ratio of AC/TC of not less than 10 may perhaps lead to more representative results, while maintaining complex AC. We therefore tried to discuss Apache Httpd, Mypaint and VSCode separately as these are the only units among the aforementioned whose average Cyclomatic Complexity rejects the null hypothesis of our statistical analysis. The reader will however note that results for Apache Httpd, Mypaint and VSCode are sometimes similar to those obtained for other case study systems. The potential impact of testing effort on the phenomenon we want to study, if there is one, is therefore unclear and should be studied. In order to draw conclusions from the data we needed to use inferential statistics because, although very informative, descriptive statistics can be misleading due to heavily skewed distributions of complexity values. We relied on standard non-parametric statistical tests; all results, except average values for three, are statistically significant. 


\section{Chapter 7 CONCLUSIONS}

On non-trivial software, the large test code base needs adequate maintenance similarly to application code. It has often been argued that test code should be simple, some authors even arguing that a method implementing a test case should be reduced to a single control flow. If this is indeed the case, application code and test code may have to be considered differently from a software maintenance point of view, despite the fact that both are source code. For instance, if a Cyclomatic Complexity value threshold has been shown through empirical evaluation on application code to warrant code refactoring and such a complexity level is un-heard of in test code, the threshold is useless as a decision mechanism to refactor test code, despite the fact that test code may nevertheless need refactoring.

In this study, we tried to confirm or refute this claim about the respective complexity of application code and test code by measuring the complexity of test code and application code of a number of large, non-trivial and well-known open source software systems.

We decided to measure the Cyclomatic Complexity of test methods and application logic methods with a commercial source code analysis software package.

We observe that descriptive statistics such as average and mean values provide interesting information but that this information can be misleading if not used with care. This is due to the fact that (test and application) code contains a very large proportion of simple methods that have a Cyclomatic Complexity of 1 or 2 and that this heavily skews frequency distributions of Cyclomatic Complexity values to the left. We therefore complemented the descriptive statistical analysis with an inferential statistical analysis, using non-parametric tests.

Our results indicate that, overall, application code is indeed statistically more complex than test code. However, contrary to the claim that test methods should be as simple as possible, to the extent that they should be reduced to a single flow of control, test code appears to be very complex in some cases. While the majority of test methods have a Cyclomatic Complexity value below 50 , which is particularly considered difficult to understand, it is, however, not rare to observe test methods with a Cyclomatic Complexity value above 100,150 or even 1000 . Above a cyclomatic complexity value of 50 , the code is typically considered practically unmaintainable. We have identified test methods with Cyclomatic Complexity values of 83, 97,156, 186, and as large as 2,097. 
Another interesting observation we made is that there is more than one line of application code for each line of test code in open source software whereas there is more than one line of test code per line of application code in industry systems we observed. Test code relative to application code appears to be different in industry and open-source. One should therefore be careful when studying open-source and attempting to generalize to industry. In determining the complexity difference in these two kinds of code (application code and test code), the kind of software really matters.

From a code maintenance point of view, although test code is statistically less complex than application code, the two seem to be relatively similar to each other to possibly justify generalization of code maintenance research results on application code to code maintenance of test code. One may however be cognisant of the typical structure of test code (e.g., set-up, execution, tear-down), which could prevent such generalization. More work is warranted to better study test code maintenance.

In this study, we have illustrated by means of empirical results from open-source software systems the correlation between software metrics. Our observations suggest that the monotonicity that exists in software metrics is not exclusively due to the kind of metrics but largely due to factors such as kind of code, kind of software and kind of metrics. While numerous studies hypothesize the existence of some form of (strong) correlation between Lines of Code (LOC) and Cyclomatic Complexity (CC), this study reveals that this observation is only true for application code and not for test code. Contrary to what most authors have extrapolated to be the rule for all source code, test code has a weak or almost no correlation between LOC and CC. We investigated an outlier claim made by some authors who argued that the extent of correlation between the two is only strong when the software is analyzed as an aggregate unit and is rather moderate in modules, however, we find that this claim is not wholly valid. At the lowest level of granularity, which is the method level, there still exist a strong correlation between Cyclomatic Complexity and Lines of Code for application code.

In other studies, some authors present a verifiable evidence of a stable relationship between Cyclomatic Complexity and Lines of Code and attempt to nullify the strength and relevance of Cyclomatic Complexity by giving merit to Lines of Code but fail to present an explanation for other kind of code (test code to be specific) and a quintessential lucid 
justification for the use of Lines of Code over Cyclomatic Complexity. We find their study open to both sides as one could as well use their results to advocate the use of CC over LOC. In our study, we have outlined reasons why CC should be preferred over LOC. Given the weak monotonicity between Cyclomatic Complexity and Lines of Code, Cyclomatic Complexity and Halstead Effort, Lines of Code and Halstead Effort for test code, we disagree that Cyclomatic Complexity and Halstead metrics are redundant as some studies suggest. Rather, we advocate for the use of Cyclomatic Complexity over Lines of Code (or both or Cylomatic density) due to reasons such as cognitive complexity, numerical complexity, adequacy of testing, interdependency and code refactoring that cannot be accounted for by Lines of Code. To have a better analysis, the two (cyclomatic Complexity and Lines of Code) and Cyclomatic density suffice as all these metrics have different objectives and algorithms for measurement. In addition, the amount of test cases on open source software and the adequacy they attain suggest most open source software do not come with enough test code, with a Production Code to Test code LOC ratio of 129:1 and a structural coverage of $40 \%$. 


\section{REFERENCES}

[1] T. McCabe, "A Complexity Measure", IEEE Transactions on Software Engineering, vol. -2, no. 4, pp. 308-320, 1976. T. McCabe, "A Complexity Measure", IEEE Transactions on Software Engineering, vol. -2, no. 4, pp. 308-320, 1976.

[2] T. McCabe and C. Butler, "Design complexity measurement and testing", Communications of the ACM, vol. 32, no. 12, pp. 1415-1425, 1989.

[3] A. Nuñez-Varela, H. Pérez-Gonzalez, F. Martínez-Perez and C. SoubervielleMontalvo, "Source code metrics: A systematic mapping study", Journal of Systems and Software, vol. 128, pp. 164-197, 2017.

[4] S. Almugrin, W. Albattah and A. Melton, "Using indirect coupling metrics to predict package maintainability and testability", Journal of Systems and Software, vol. 121, pp. 298-310, 2016.

[5] S. Yu and S. Zhou, "A survey on metric of software complexity," 2010 2nd IEEE International Conference on Information Management and Engineering, 2010.

[6] T. Hariprasad, G. Vidhyagaran, K. Seenu, and C. Thirumalai, "Software complexity analysis using halstead metrics,". International Conference on Trends in Electronics and Informatics (ICEI), 2017.

[7] R. Prather, "Software metrics: A rigorous approach: Fenton, NE. 337 pages, isbn 0-412-40440-0; Published by Chapman and Hall, London", Software Testing, Verification and Reliability, vol. 1, no. 3, pp. 43-43, 1991.

[8] N. Fenton and J. Bieman, "Software Metrics," Chapman \& Hall/CRC Innovations in Software Engineering and Software Development Series, Oct. 2014.

[9] Halstead, M.H. "Elements of Software Science," NorthHolland, 1997.

[10] Robert V. Binder, Testing object-oriented systems: models, patterns, and tools, Addison-Wesley Longman Publishing Co., Inc., Boston, MA, 1999.

[11] R. Binder, "Object-oriented software testing", Communications of the ACM, vol. 37, no. 9, pp. 28-29, 1994.

[12] A. Mili and F. Tchier, Software testing. Hoboken, NJ: Wiley, 2015.

[13] Oliveira, C. D., Fong, E. N., \& Black, P. E. (2017). Impact of code complexity on software analysis. doi:10.6028/nist.ir.8165

[14] Tripathy, P. and Naik, K. (2014). "Taxonomy of Software Maintenance and Evolution". In Software Evolution and Maintenance (eds P. Tripathy and K. Naik). doi:10.1002/9781118964637.ch2

[15] "Parasoft | Parasoft - Automated Software Testing", Parasoft.com. [Online]. Available: https://www.parasoft.com/. [Accessed: 11- May- 2018].

[16] "SciTools.com", Scitools.com, 2018. [Online]. Available: https://scitools.com/. [Accessed: 11- May- 2018].

[17] "Testwell", Testwell.fi, 2018. [Online]. Available: http://www.testwell.fi/. [Accessed: 11- May- 2018].

[18] "Supported Languages | SciTools.com", Scitools.com, 2018. [Online]. Available: https://scitools.com/feature/supported-languages/. [Accessed: 11- May- 2018].

[19] Plot.ly, 2018. [Online]. Available: https://plot.ly/. [Accessed: 11- May- 2018]. 
[20] "Metrics 1.3.6", Metrics.sourceforge.net, 2018. [Online]. Available: http://metrics.sourceforge.net/. [Accessed: 11- May- 2018].

[21] D. Kafura and G. Reddy, "The Use of Software Complexity Metrics in Software Maintenance", IEEE Transactions on Software Engineering, vol. -13, no. 3, pp. 335-343, 1987.

[22] J. Weissman and J. King, "What makes a project big?" Software Engineering StackExchange,2020.[Online].Available:

https://softwareengineering.stackexchange.com/questions/17177/what-makes-aproject-big.

[23] M. J. P. v. d. Meulen and M. A. Revilla, "Correlations between Internal Software Metrics and Software Dependability in a Large Population of Small C/C++ Programs," The 18th IEEE International Symposium on Software Reliability (ISSRE '07), Trollhattan, 2007, pp. 203-208.

[24] G. Gill and C. Kemerer, "Cyclomatic complexity density and software maintenance productivity", IEEE Transactions on Software Engineering, vol. 17, no. 12, pp. 1284-1288, 1991.

[25] S. Henry and D. Kafura, "Software Structure Metrics Based on Information Flow", IEEE Transactions on Software Engineering, vol. -7, no. 5, pp. 510-518, 1981.

[26] P. Hamer and G. Frewin, "M.H. Halstead's Software Science - a critical examination", Dl.acm.org, 2018. [Online]. Available: https://dl.acm.org/citation.cfm?id=800254.807762. [Accessed: 11- May- 2018].

[27] Sarrab, M \& Rehman, O.M.H. 2013, 'Selection criteria of open source software: First stage for adoption' International Journal of Information Processing and Management, vol. 4, no. 4, pp. 51-58. DOI: 10.4156/ijipm.vol4.issue4.6

[28] D. W. Schuster, "Selection Process for Free Open Source Software," Advances in Library and Information Science Library Technology Funding, Planning, and Deployment, pp. 55-71

[29] D. Taibi, "Can Opinion Mining Techniques Help to Select Open Source Software?", International Journal of Computer \& Software Engineering, vol. 1, no. $1,2016$.

[30] P. Bürkner, P. Doebler and H. Holling, "Optimal design of the Wilcoxon-MannWhitney-test", Biometrical Journal, vol. 59, no. 1, pp. 25-40, 2016.

[31] L. E. Moses, "Wilcoxon-Mann-Whitney Test: Definition and Example," Wiley StatsRef: Statistics Reference Online, 2014.

[32] Meeker, W. Q., Hahn, G. J. and Escobar, L. A. (2017). "Methods for Calculating Statistical Intervals for a Normal Distribution.” In Statistical Intervals (eds W. Q. Meeker, G. J. Hahn and L. A. Escobar). doi:10.1002/9781118594841.ch4

[33] Norman Fenton, James Bieman, Software Metrics: "A Rigorous and Practical Approach,” Third Edition, CRC Press, Inc., Boca Raton, FL, 2014

[34] D. Landman, A. Serebrenik, E. Bouwers, J. J. Vinju, "Empirical analysis of the relationship between $\mathrm{CC}$ and SLOC in a large corpus of Java methods and C functions", Journal of Software: Evolution and Process, vol. 28, no. 7, 2016. 
[35] R. K. Lind, K. Vairavan, "An Experimental Investigation of Software Metrics and Their Relationship to Software Development Effort", IEEE Transactions on Software Engineering, New Jersey - USA, 1989, pp. 649-653.

[36] S. R. Chidamber, C. F. Kemerer, A Metrics Suite for Object Oriented Design, IEEE Transactions on Software Engineering, v.20 n.6, p.476-493, June 1994

[37] Tashtoush, Yahya \& Al-Maolegi, Mohammed \& Arkok, Bassam. (2014). "The Correlation among Software Complexity Metrics with Case Study". International Journal of Advanced Computer Research. 4.

[38] Abran, A. (2010). Halstead's Metrics: Analysis of Their Designs. In Software Metrics and Software Metrology, A. Abran (Ed.)

[39] Meszaros, Gerard. XUnit Test Patterns: Refactoring Test Code. (2018).

[40] D. Athanasiou, A. Nugroho, J. Visser and A. Zaidman, "Test Code Quality and Its Relation to Issue Handling Performance," in IEEE Transactions on Software Engineering, vol. 40, no. 11, pp. 1100-1125, Nov. 12014

[41] Boris Beizer, Software testing techniques (2nd ed.), Van Nostrand Reinhold Co., New York, NY, 1990.

[42] C. Wohlin, P. Runeson, M. Höst, M. C. Ohlsson, B. Regnell, and A. Wesslén, Experimentation in software engineering, vol. 9783642290. 2012.

[43] S. Ajami, Y. Woodbridge and D. Feitelson, "Syntax, predicates, idioms — what really affects code complexity?", Empirical Software Engineering, vol. 24, no. 1, pp. 287-328, 2018. Available: 10.1007/s10664-018-9628-3.

[44] Mamun, Md Abdullah \& Berger, Christian \& Hansson, Jörgen. (2017). Correlations of software code metrics: an empirical study. 255-266. 10.1145/3143434.3143445.

[45] M. Mamun, C. Berger and J. Hansson, "Effects of measurements on correlations of software code metrics", Empirical Software Engineering, 2019. Available: 10.1007/s 10664-019-09714-9.

[46] Sallie Henry, Dennis Kafura, and Kathy Harris. 1981. On the Relationships Among Three Software Metrics. In Proceedings of the 1981 ACM Workshop/Symposium on Measurement and Evaluation of Software Quality. ACM, New York, NY, USA, 81-88. https://doi.org/10.1145/800003.807911

[47] M. J. P. v d Meulen and M. A. Revilla. 2007. Correlations between Internal Software Metrics and Software Dependability in a Large Population of Small $\mathrm{C} / \mathrm{C}++$ Programs. In The 18th IEEE International Symposium on Software Reliability (ISSRE '07). 203-208. https://doi.org/10.1109/ISSRE.2007.12

[48] S. Saini, S. Sharma, and R. Singh. 2015. Better utilization of correlation between metrics using Principal Component Analysis (PCA). In 2015 Annual IEEE India Conference (INDICON). 1-6. https://doi.org/10.1109/INDICON.2015.7443299

[49] Davy Landman, Alexander Serebrenik, Eric Bouwers, and Jurgen J. Vinju. 2016.Empirical analysis of the relationship between CC and SLOC in a large corpus of Java methods and C functions. Journal of Software: Evolution and Process 28, 7 (July 2016), 589-618. https://doi.org/10.1002/smr.1760

[50] Muslija, A., \& Enoiu, E. P. (2018). On the correlation between testing effort and software complexity metrics. doi:10.7287/peerj.preprints.27312v1 
[51] Graylin Jay, Joanne E. Hale, Randy K. Smith, David Hale, Nicholas A. Kraft, and Charles Ward. 2009. Cyclomatic Complexity and Lines of Code: Empirical Evidence of a Stable Linear Relationship. Journal of Software Engineering and Applications 02, 03 (Oct. 2009), 137. https://doi.org/10.4236/jsea.2009.23020

[52] S. Devlin, R. Gnanadesikan and J. Kettenring, "Robust Estimation and Outlier Detection with Correlation Coefficients", Biometrika, vol. 62, no. 3, p. 531, 1975. Available: $10.2307 / 2335508$.

[53] Wilcox, R. (2017). Some Multivariate Methods. Introduction to Robust Estimation and Hypothesis Testing, 235-318. doi:10.1016/b978-0-12-804733-0.00006-8

[54] Wilcox, R. (2017). Correlation and Tests of Independence. Introduction to Robust Estimation and Hypothesis Testing, 485-516. doi:10.1016/b978-0-12-8047330.00009-3

[55] Puth, M., Neuhäuser, M., \& Ruxton, G. D. (2015). Effective use of Spearmans and Kendalls correlation coefficients for association between two measured traits. Animal Behaviour, 102, 77-84. doi:10.1016/j.anbehav.2015.01.010

[56] Prion, S., \& Haerling, K. A. (2014). Making Sense of Methods and Measurement: Spearman-Rho Ranked-Order Correlation Coefficient. Clinical Simulation in Nursing, 10(10), 535-536. doi:10.1016/j.ecns.2014.07.005

[57] W. Zhang, Z. Wei, B. Wang and X. Han, "Measuring mixing patterns in complex networks by Spearman rank correlation coefficient", Physica A: Statistical Mechanics and its Applications, vol. 451, pp. 440-450, 2016. Available: 10.1016/j.physa.2016.01.056.

[58] Prion, S., \& Haerling, K. A. (2014). Making Sense of Methods and Measurement: Pearson Product-Moment Correlation Coefficient. Clinical Simulation in Nursing, 10(11), 587-588. doi:10.1016/j.ecns.2014.07.010

[59] Taylor, R. (1990). Interpretation of the Correlation Coefficient: A Basic Review. Journal of Diagnostic Medical Sonography, 6(1), 35-39. doi:10.1177/875647939000600106

[60] Halsteads Metrics: Analysis of Their Designs. (2010). Software Metrics and Software Metrology, 145-159. doi: 10.1002/9780470606834.ch7

[61] Steinskog DJ. A cautionary note on the use of the Kolmogorov-Smirnov test for normality. American Meteor Soc. 2007;135:1151-7

[62] D'Agostino, R.B., Belanger, A., Ralph, B. and D'Agostino Jr., R.B. (1990) A Suggestion for Using Powerful and Informative Tests of Normality. The American Statistician, 44, 316-321.

[63] Thode HJ. Testing for normality. New York: Marcel Dekker; 2002

[64] Ghasemi, A., \& Zahediasl, S. (2012). Normality tests for statistical analysis: a guide for non-statisticians. International journal of endocrinology and metabolism, 10(2), 486-489. doi:10.5812/ijem.3505

[65] Sawyer, Steven. (2009). Analysis of Variance: The Fundamental Concepts. Journal of Manual \& Manipulative Therapy. 17. 27E-38E. 10.1179/jmt.2009.17.2.27E.

[66] Kochhar, Pavneet Singh \& Bissyandé, Tegawendé \& Lo, David \& Jiang, Lingxiao. (2013). An Empirical Study of Adoption of Software Testing in Open Source 
Projects. Proceedings of the International Symposium on the Physical and Failure Analysis of Integrated Circuits, IPFA. 103-112. 10.1109/QSIC.2013.57.

[67] Kim, Tae. (2017). Understanding one-way ANOVA using conceptual figures. Korean Journal of Anesthesiology. 70. 22. 10.4097/kjae.2017.70.1.22.

[68] Fante, R.L,IEEE. Central limit theorem: Use with caution. Transactions on Aerospace and Electronic Systems, Publication Date: April 2001,Volume: 37 , Issue: 2,pp: 739 - 740 .

[69] The central limit theorem and low-pass filters",Engelberg,Proceedings of the 2004 11th IEEE International Conference on Electronics, Circuits and Systems, 2004,1315 Dec. 2004,pp-65-68.

[70] J. Perolat, I. Couso, K. Loquin and O. Strauss, "Generalizing the Wilcoxon ranksum test for interval data", International Journal of Approximate Reasoning, vol. 56, pp. 108-121, 2015. Available: 10.1016/j.ijar.2014.08.001.

[71] F.Wilcoxon, Individual Comparisons by Ranking Methods, Biom.Bull.1(1945)8083.

[72] Coleman, D.; Lowther, B.; and Oman, P.; Using Metrics to Evaluate Software System Maintainability, IEEE Computer, Vol. 27(8), pp. 44-49, Aug. 1994.

[73] Liso, A., Software Maintainability Metrics Model: An Improvement in the Coleman-Oman Model," Crosstalk, Aug. 2001, pp. 15-17.

[74] Coleman, D.; Lowther, B.; and Oman, P.; The Application of Software Maintainability Models on Industrial Software Systems, University of Idaho, Software Engineering Test Lab, Report No. 93-03 TR, Nov. 1993.

[75] Oman, P. and Hagemeister, J., Constructing and Testing of Polynomials Predicting Software Maintainability, Journal of Systems and Software, Vol. 24(3) 251-266, March 1994.

[76] Welker, K.D. and Oman, P., Software Maintainability Metrics Models in Practice, Crosstalk, Vol. 8, pp. 19-23 Nov./Dec. 1995, www.stsc.hill.af.mil /crosstalk/1995/nov/maintain.asp

[77] K. D. Welker, "Software Maintainability Index Revisited," CrossTalk - J. of Defense Softw. Eng, 2001

[78] Van Albada SJ, Robinson PA. Transformation of arbitrary distributions to the normal distribution with application to EEG test-retest reliability (2007) J Neurosci Methods 161: 205-211, DOI: 10.1016/j.jneumeth.2006.11.004

[79] "Is 1000 lines of code considered small for a personal project? Quora", Quora.com, 2020. [Online]. Available: https://www.quora.com/Is-1000lines-of-code-considered-small-for-a-personal-project. [Accessed: 17- Jan- 2020].

[80] G. Gill and C. Kemerer, "Cyclomatic complexity density and software maintenance productivity", IEEE Transactions on Software Engineering, vol. 17, no. 12, pp. 1284-1288, 1991. Available: 10.1109/32.106988 


\section{APPENDICES}

Appendix A Distributions of cyclomatic complexity values for objects.

\section{A.1 Sample representative distributions}

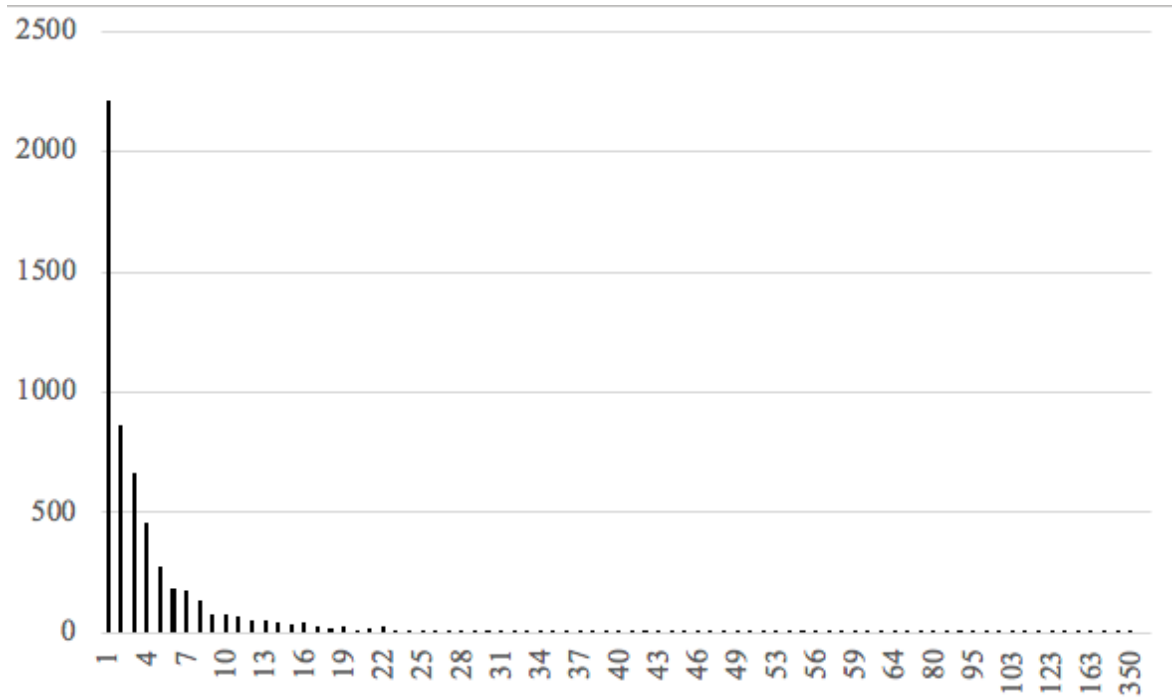

Figure 2. Distribution of cyclomatic complexity values for Apache Httpd AC

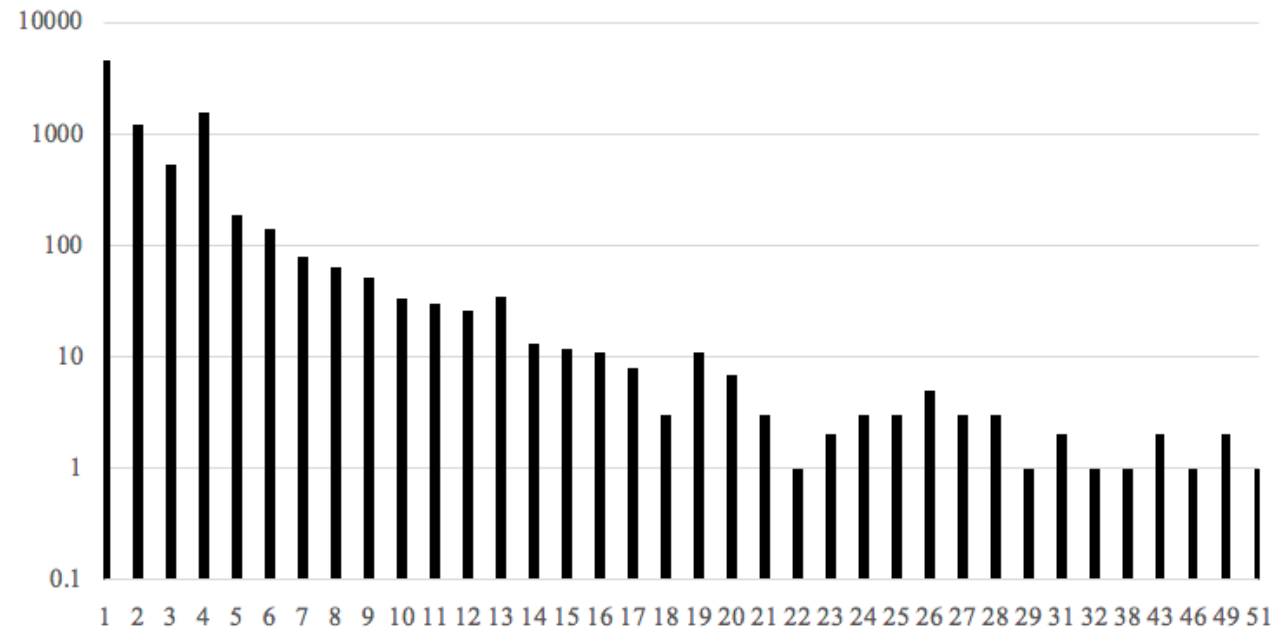

Figure 3. Distribution of Cyclomatic complexity values for Apache Httpd TC (logarithmic scale) 


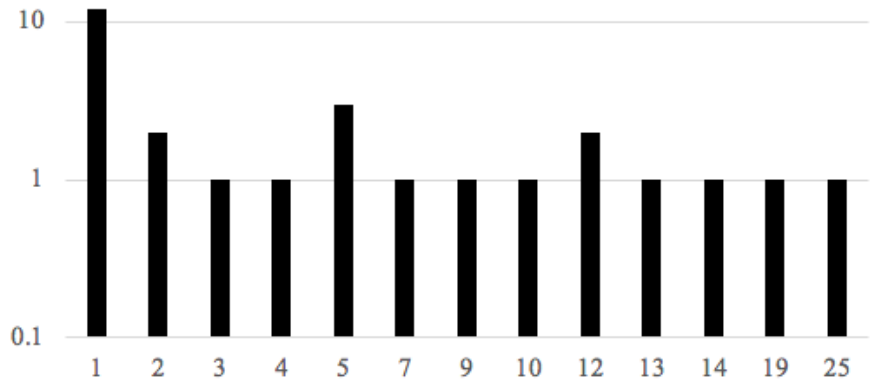

Figure 4. Distribution of cyclomatic complexity values for JFreechart AC (logarithmic scale)

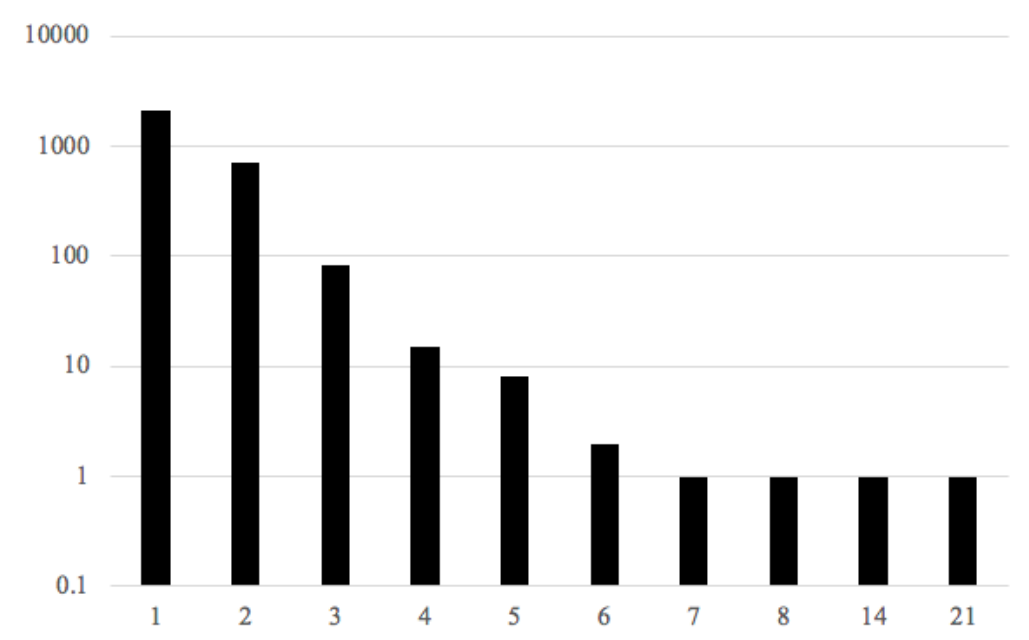

Figure 5. Distribution of cyclomatic complexity values for JFreechart TC (logarithmic scale) 


\section{A.2 Boxplots for all objects}

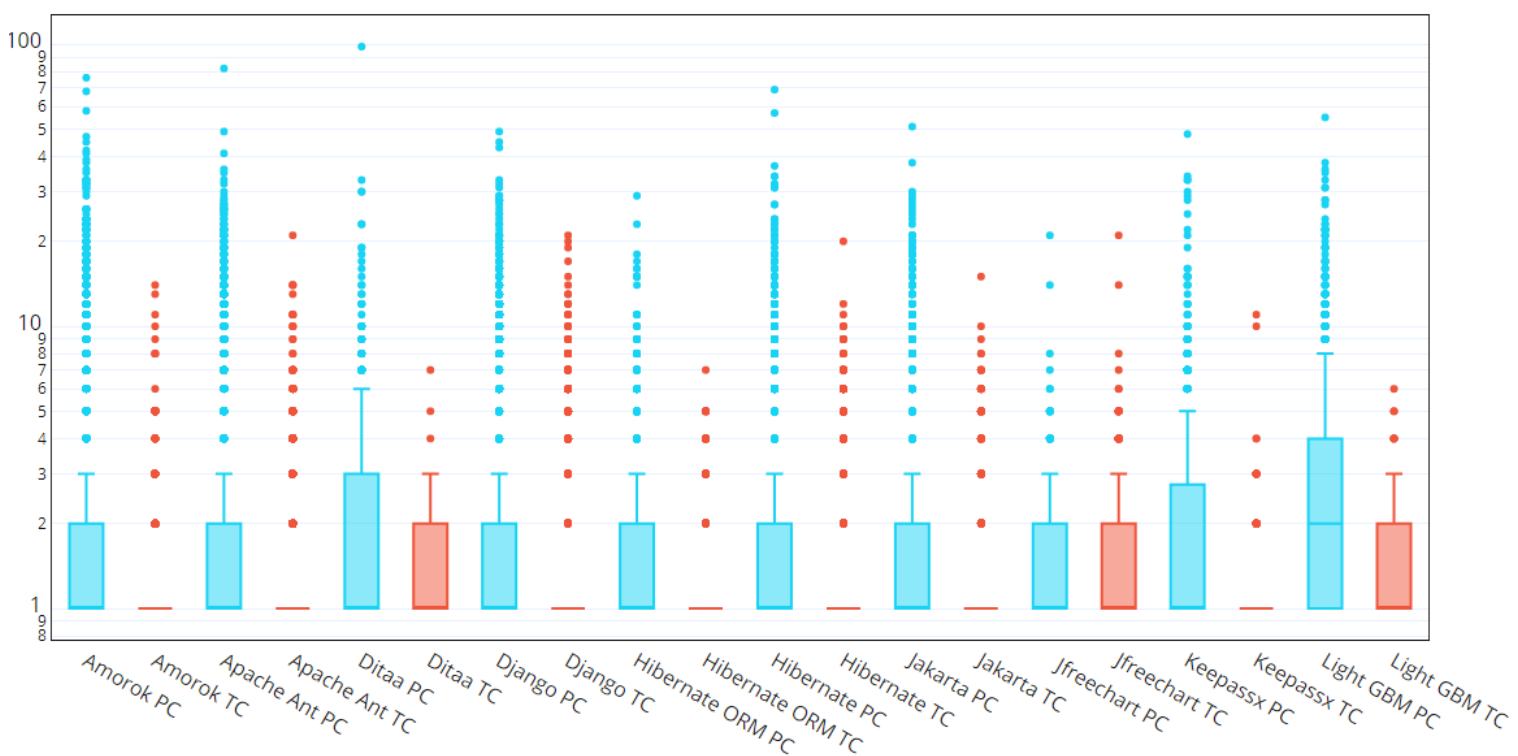

Figure 6. Box Plot of $\mathrm{CC}$ for objects with method $\mathrm{CC}$ values in range $[1,100]$.

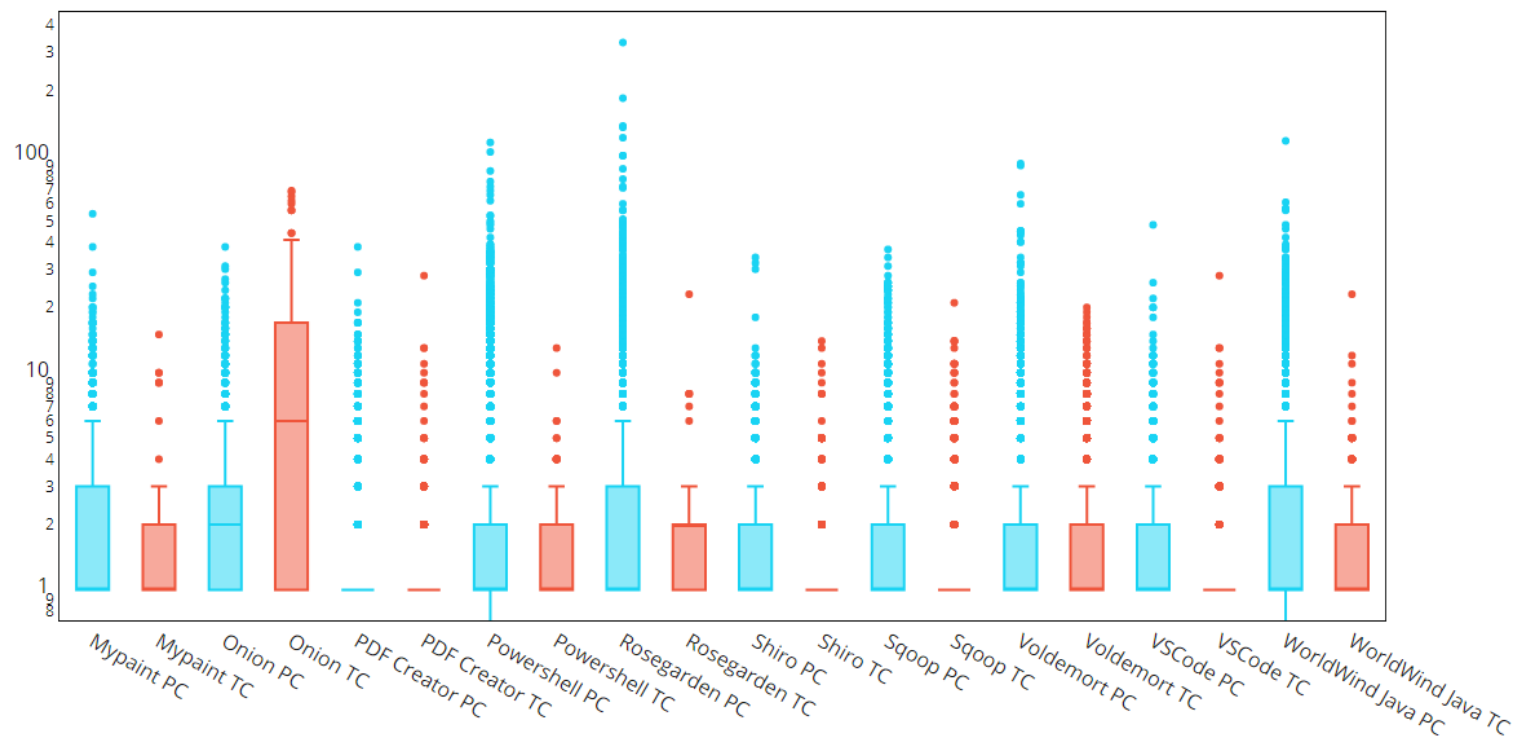

Figure 7. Box Plot of $\mathrm{CC}$ for objects with method $\mathrm{CC}$ values in range $[1,500]$. 


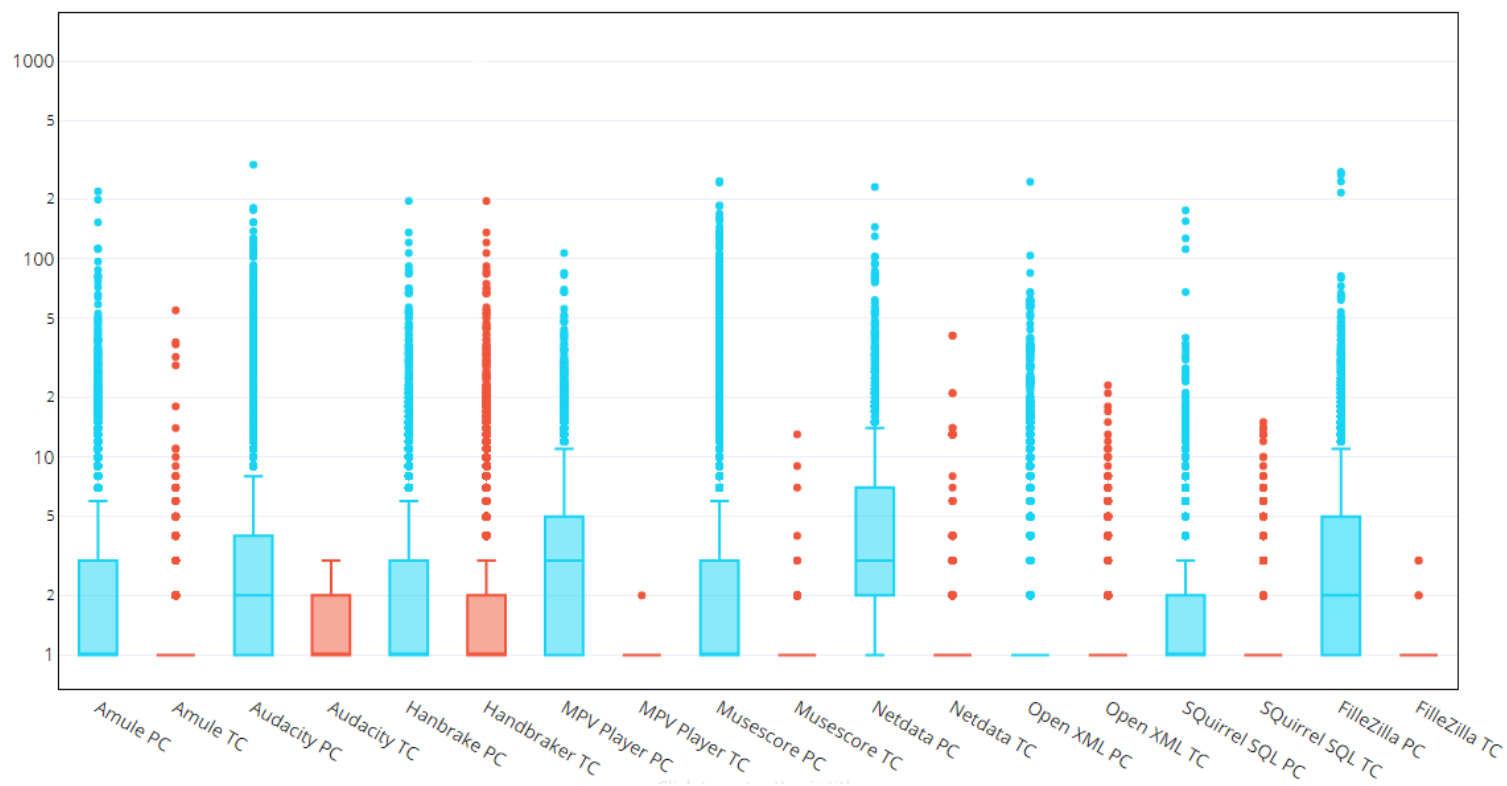

Figure 8. Box Plot of $\mathrm{CC}$ for objects with method $\mathrm{CC}$ values in range $[1,1000]$.

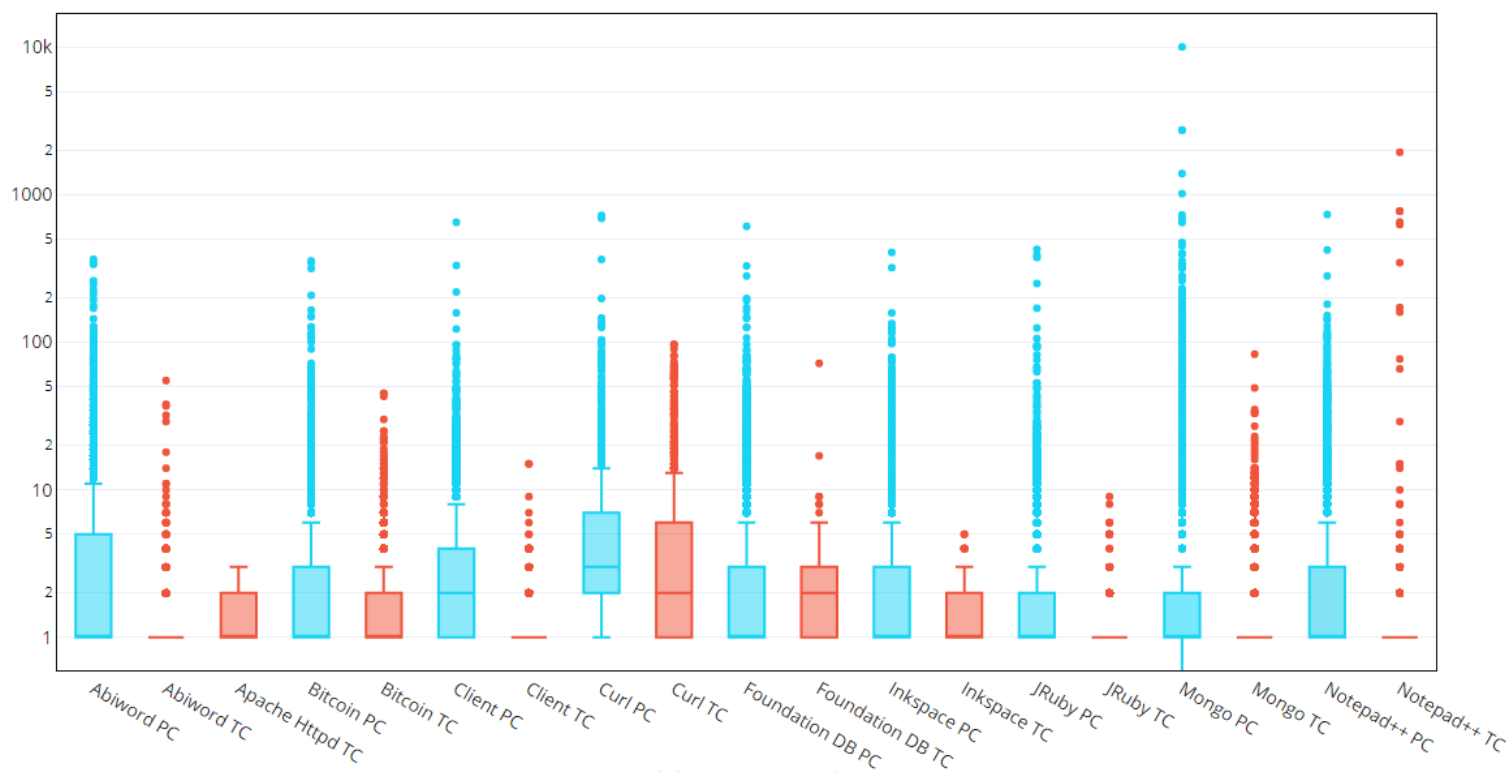

Figure 9. Box Plot of CC for objects with method $\mathrm{CC}$ values in range $[1,10000]$. 
Appendix B : LINEAR SCALE ZOOMED IN ON THE LEFT QUADRANT

\section{B.1 LINEAR SCALE LINEAR SCALE ZOOMED IN ON THE LEFT QUADRANT (PRODUCTION CODE)}

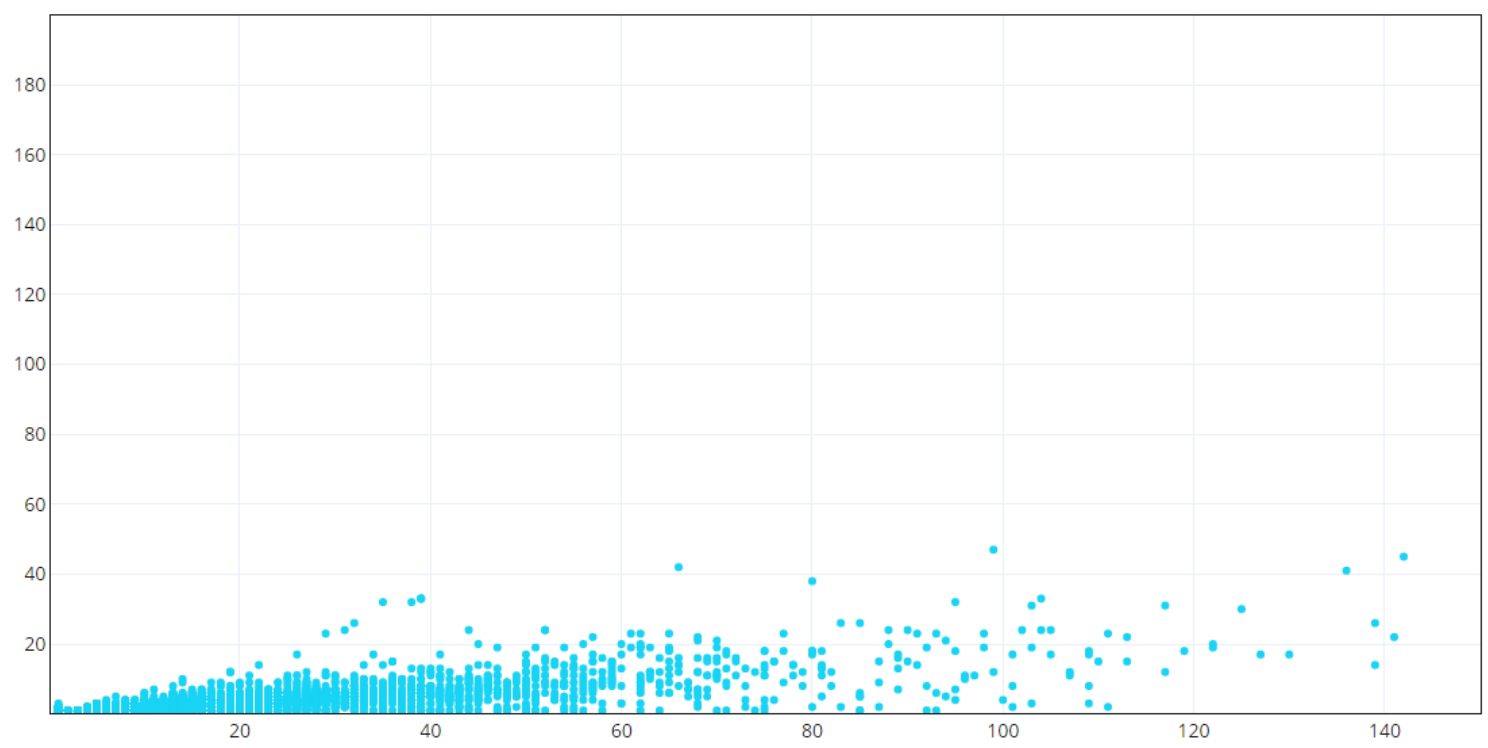

Figure 10. Scatter Plot of LOC (x) vs CC (y) for Amorok, zoomed into the bottom left quadrant.

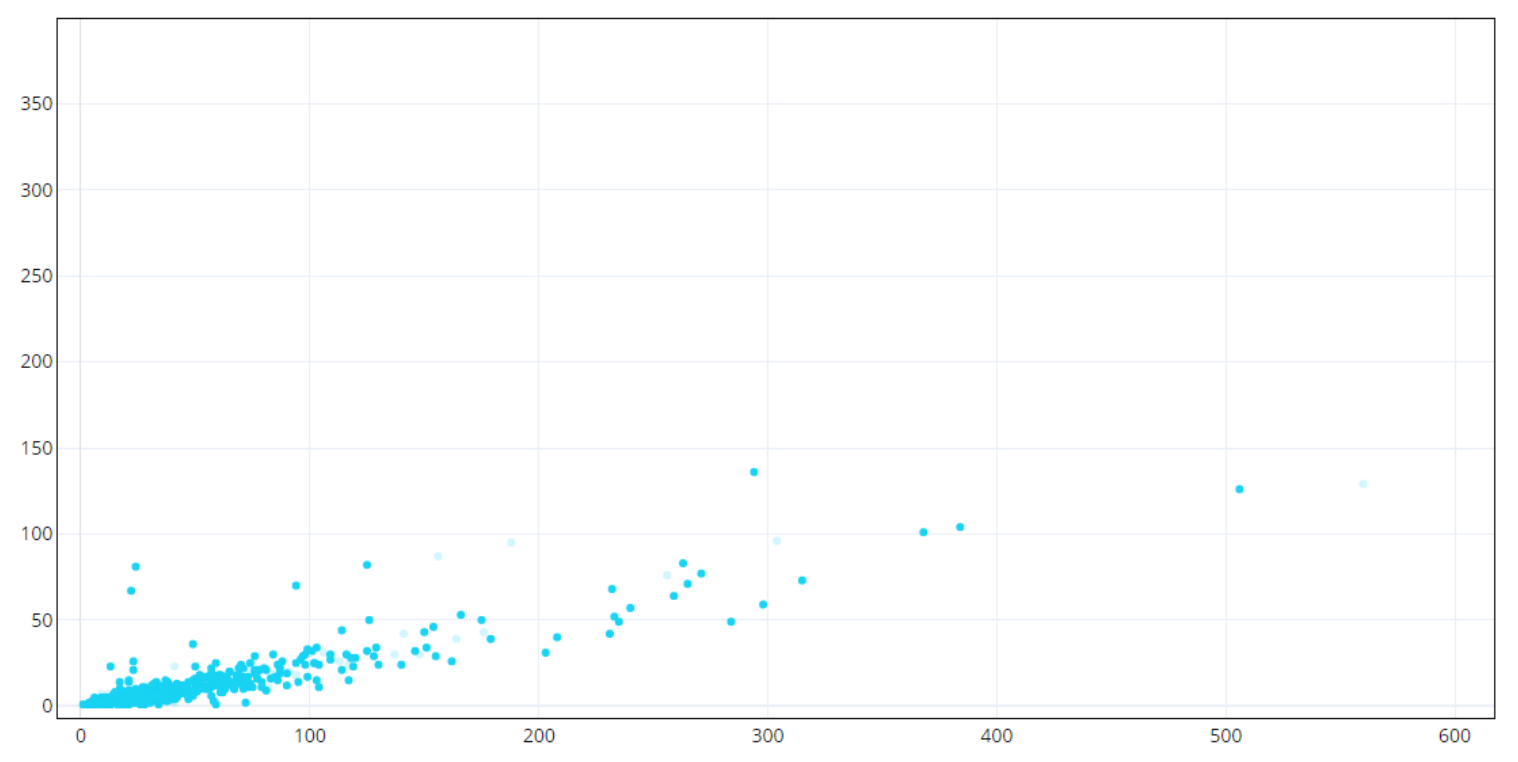

Figure 11. Scatter Plot of LOC (x) vs CC (y) for Curl, zoomed into the bottom left quadrant. 


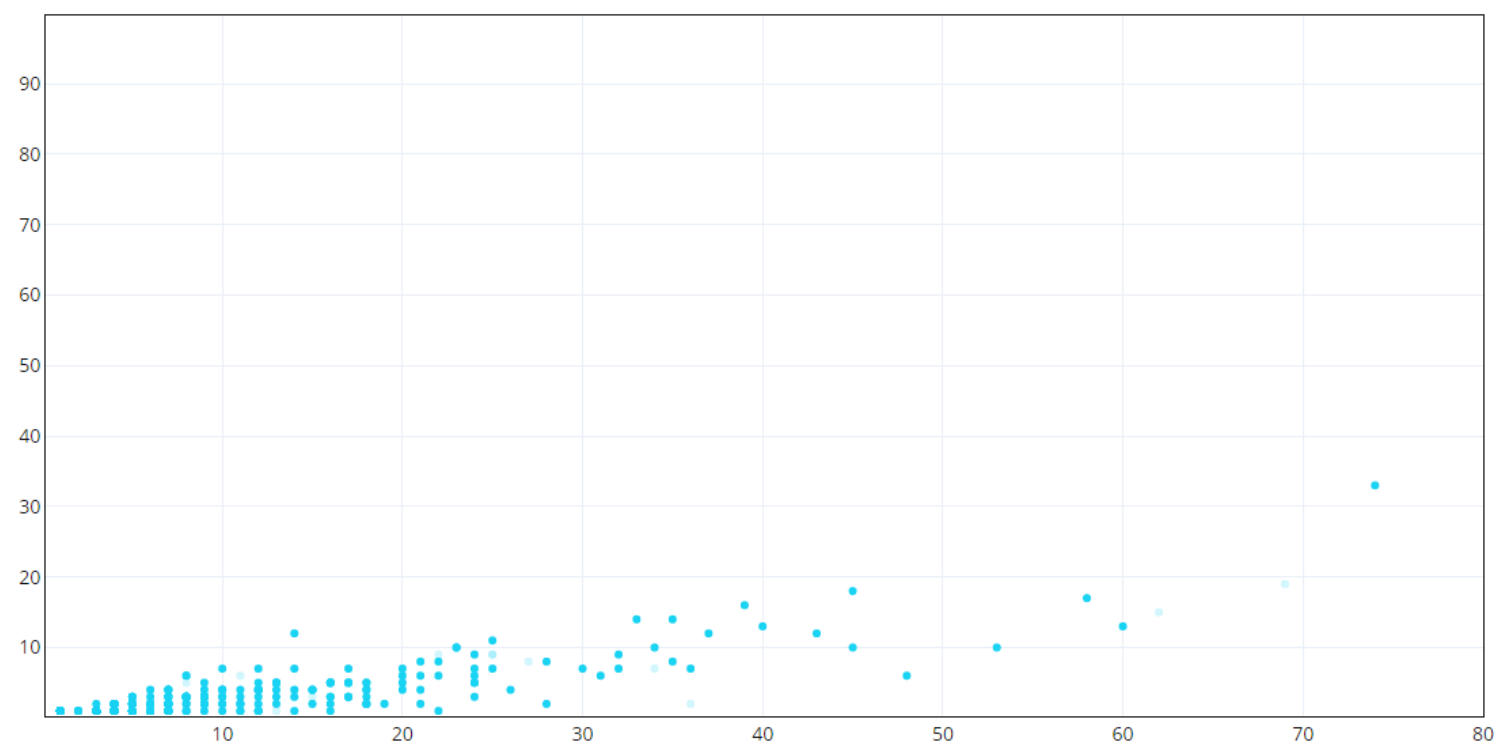

Figure 12. Scatter Plot of LOC (x) vs CC (y) for Ditaa, zoomed into the bottom left quadrant.

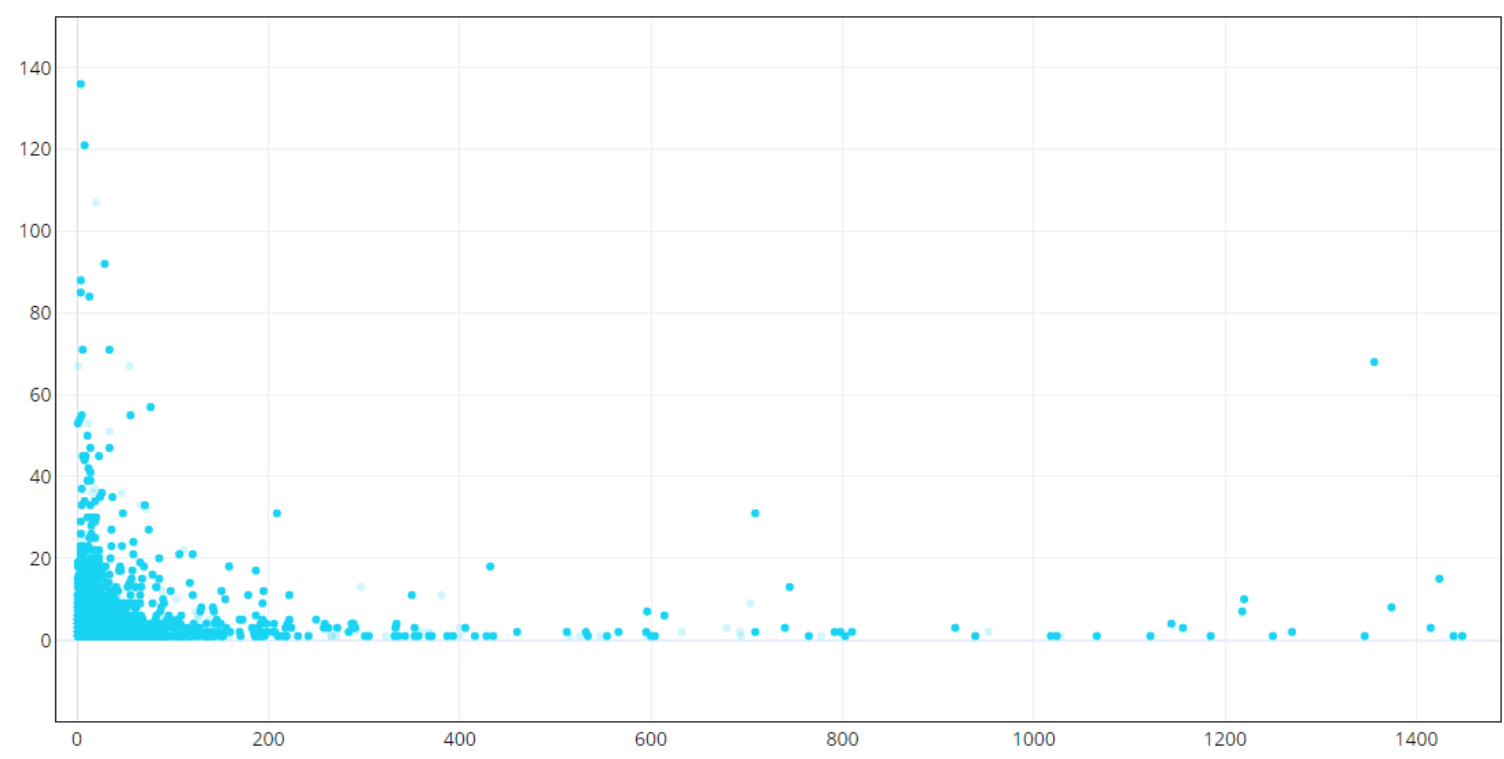

Figure 13. Scatter Plot of LOC (x) vs CC (y) for Handbrake, zoomed into the bottom left quadrant. 


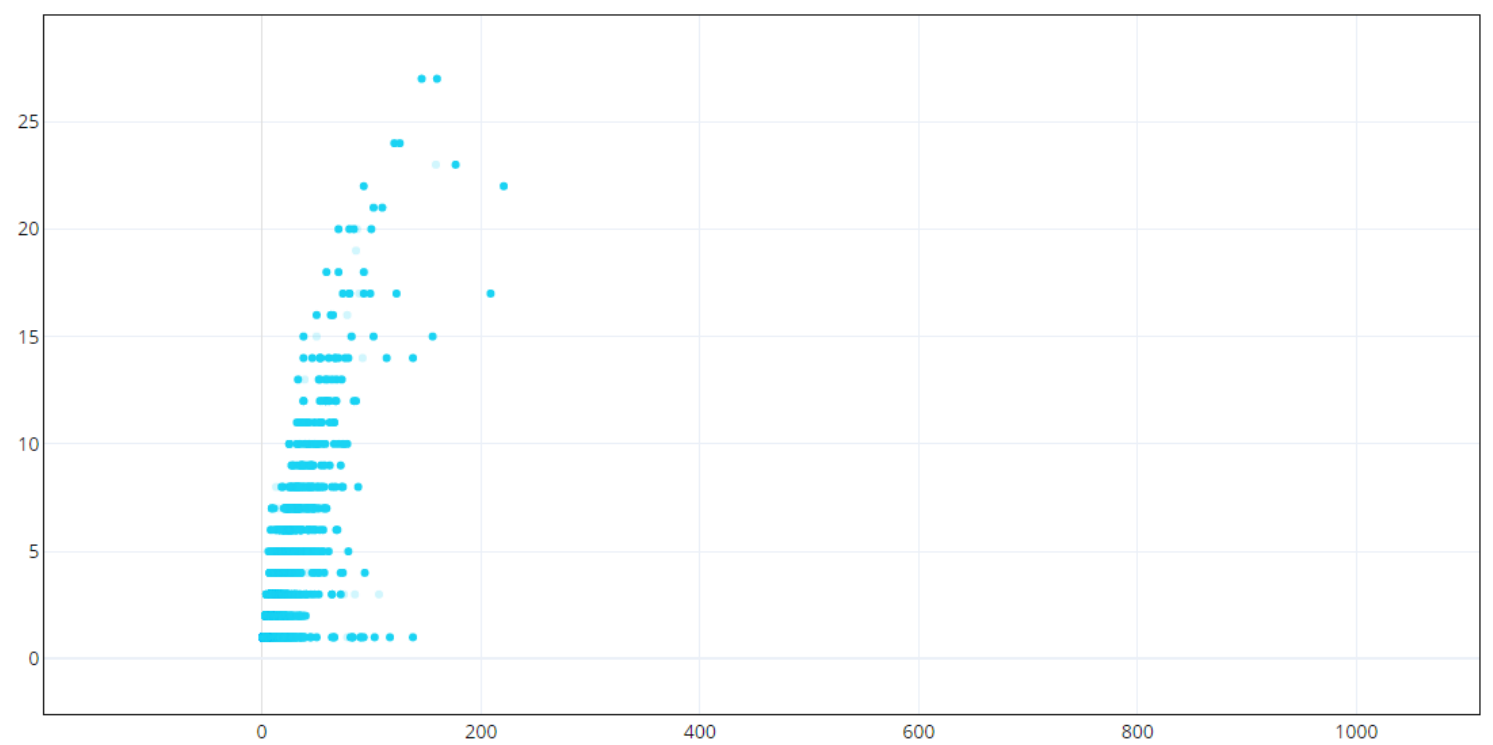

Figure 14. Scatter Plot of LOC (x) vs CC (y) for Hibernate, zoomed into the bottom left quadrant.

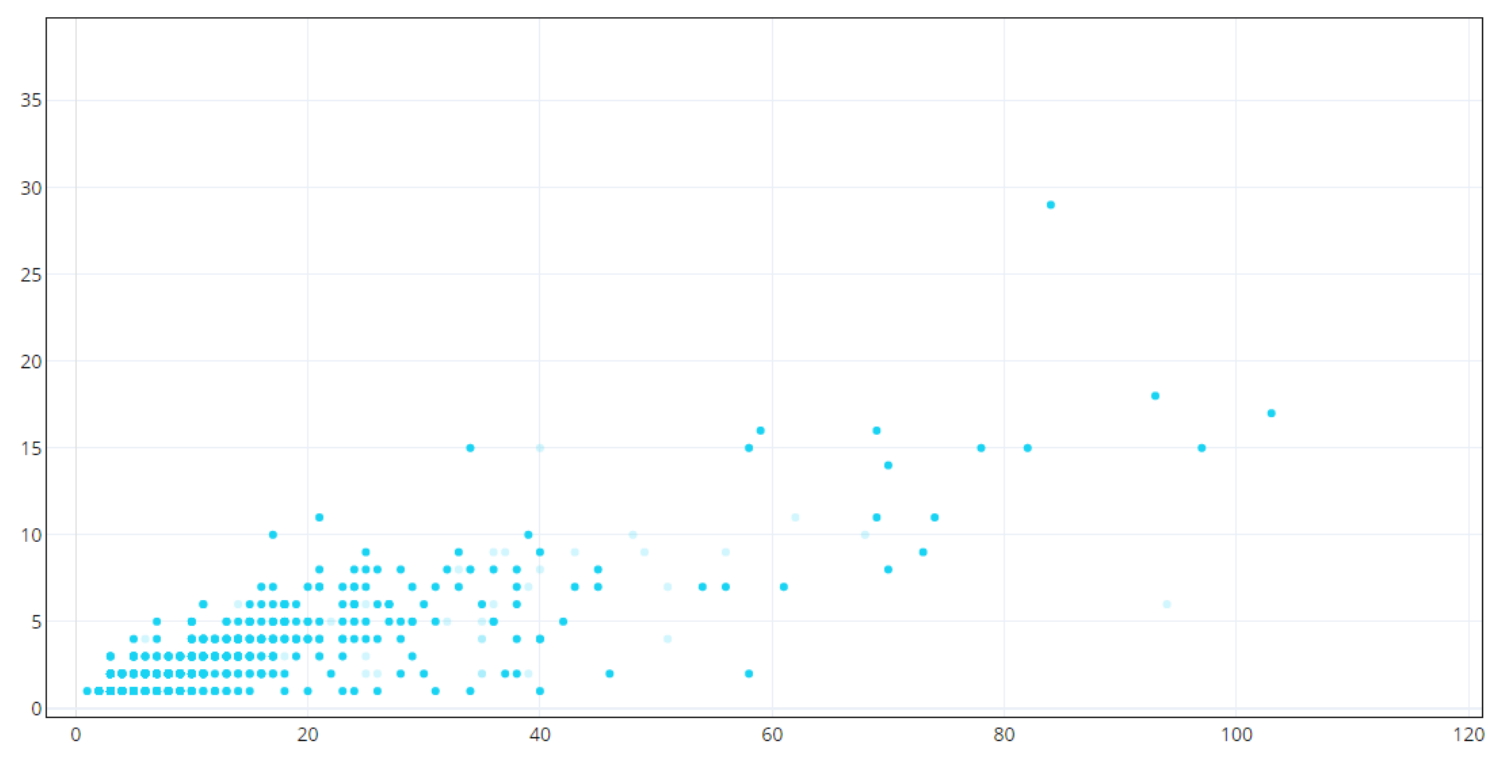

Figure 15. Scatter Plot of LOC (x) vs CC (y) for Hibernate ORM, zoomed into the bottom left quadrant. 


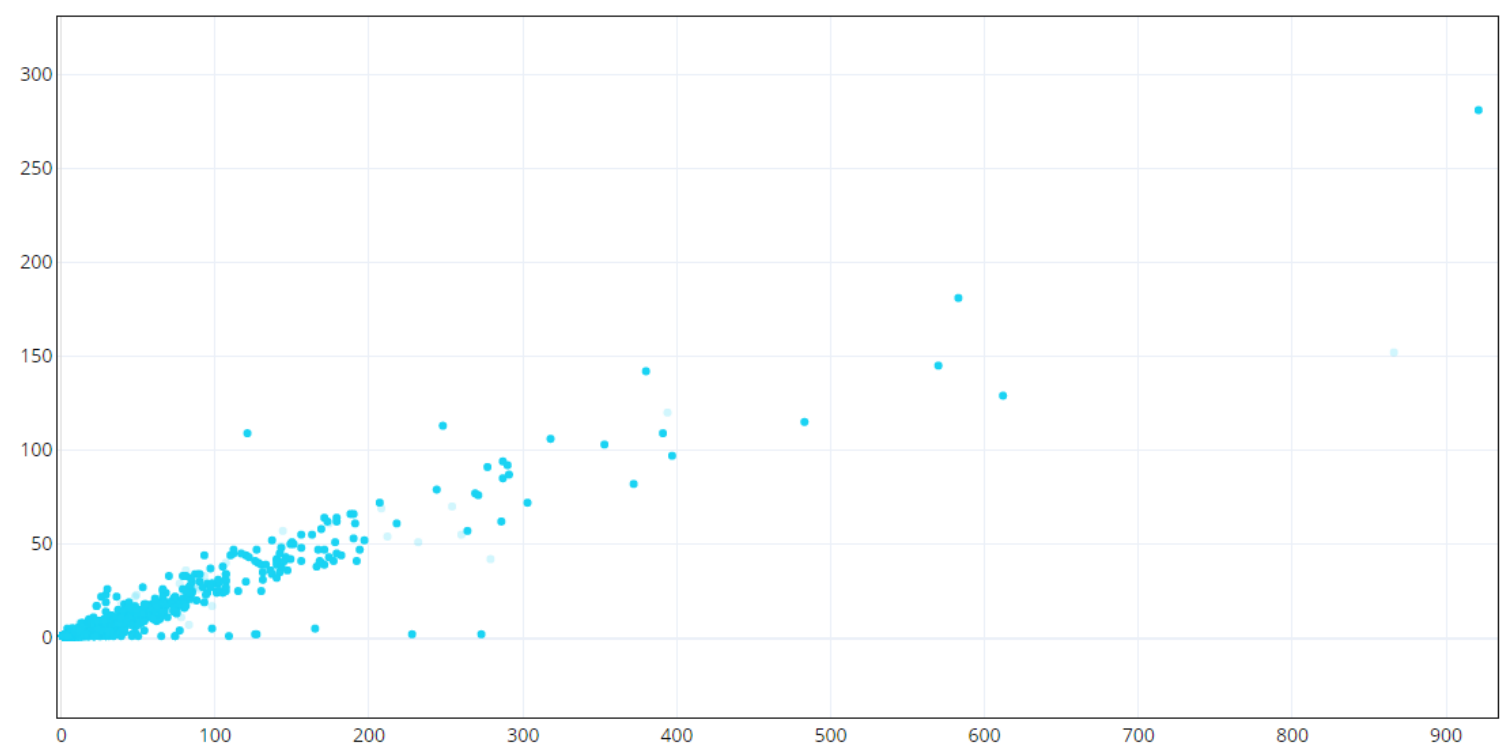

Figure 16. Scatter Plot of LOC (x) vs CC (y) for Notepad++, zoomed into the bottom left quadrant.

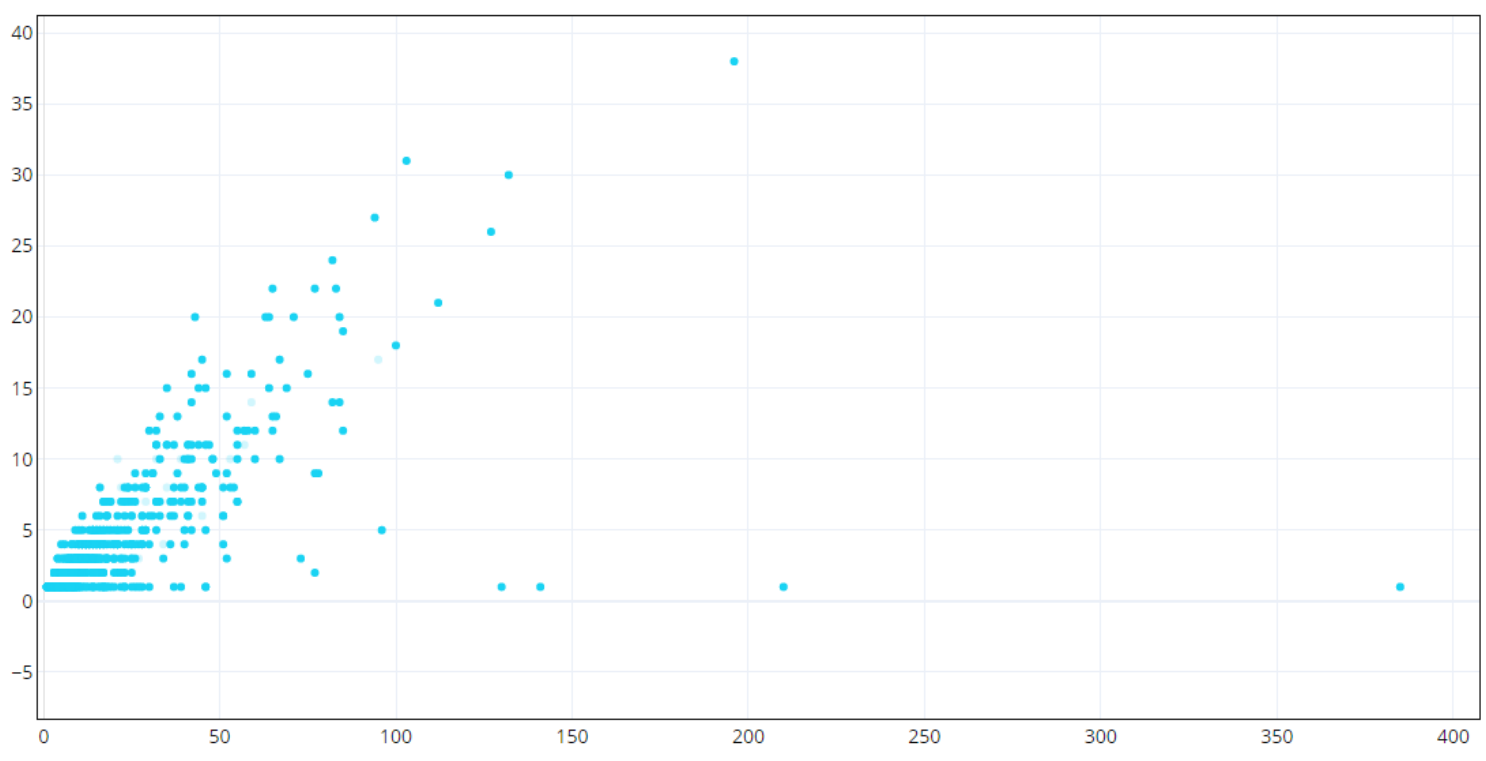

Figure 17. Scatter Plot of LOC (x) vs CC (y) for Onion, zoomed into the bottom left quadrant. 


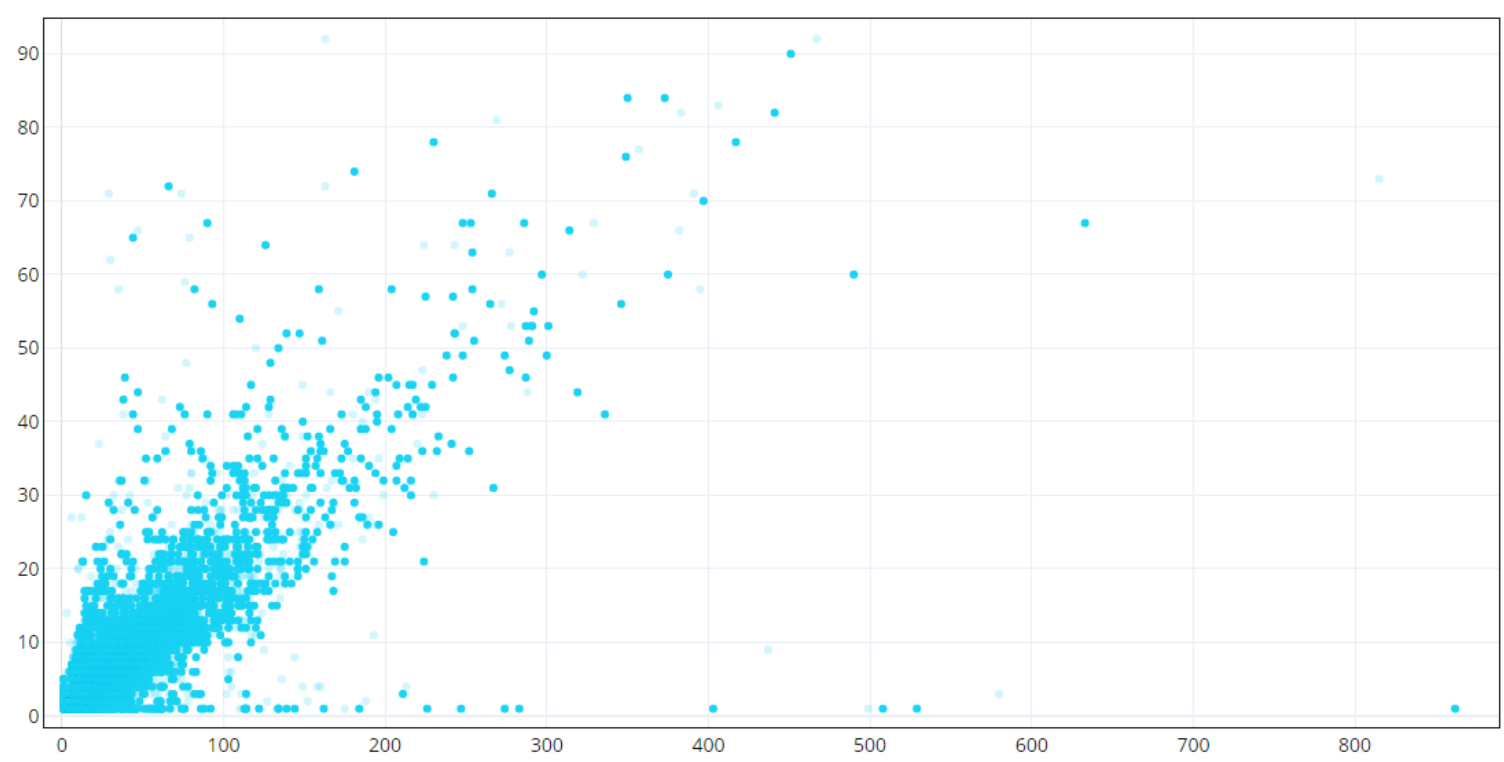

Figure 18. Scatter Plot of LOC (x) vs CC (y) for VLC, zoomed into the bottom left quadrant.

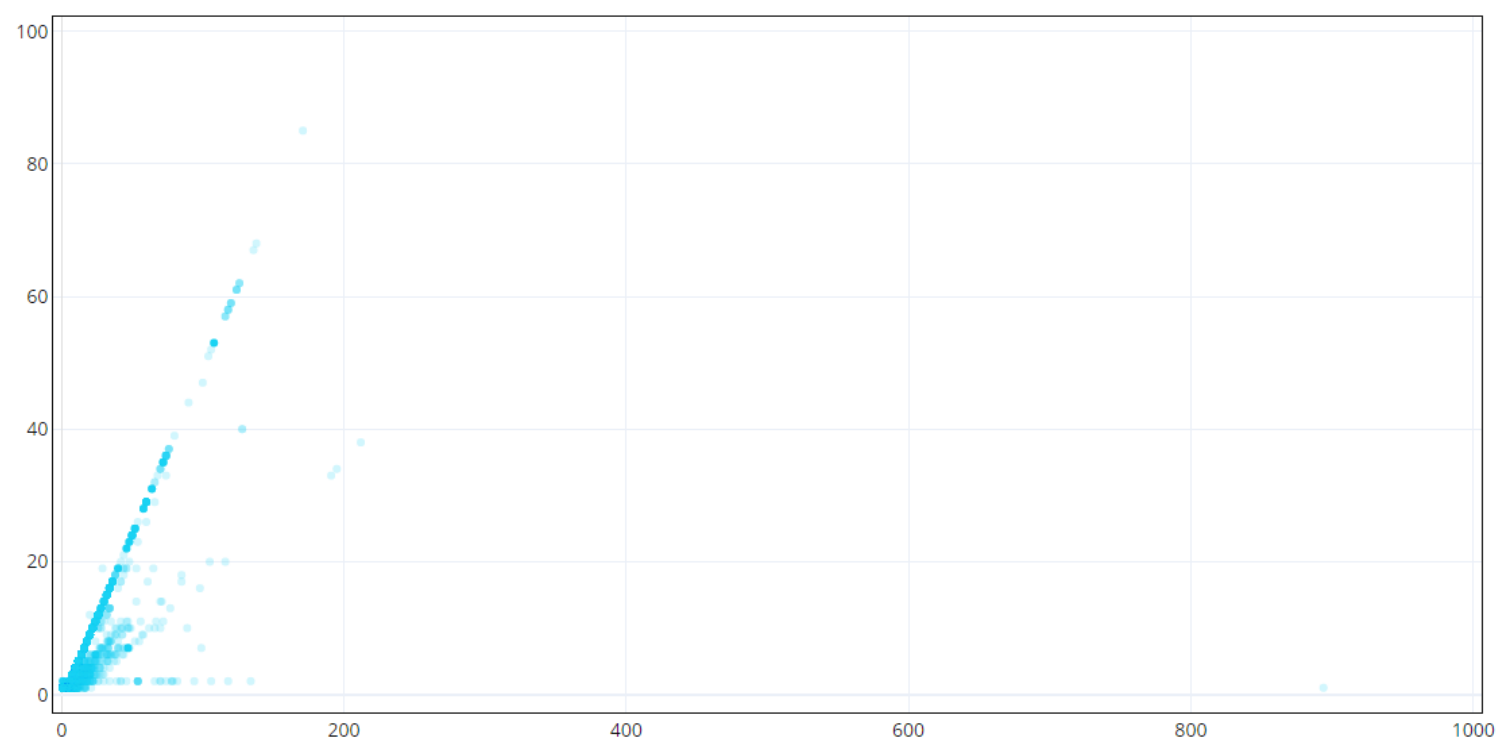

Figure 19. Scatter Plot of LOC (x) vs CC (y) for Open XML (PC), zoomed into the bottom left quadrant. 


\section{B.2 LINEAR SCALE ZOOMED IN ON THE LEFT QUADRANT (PRODUCTION CODE)}

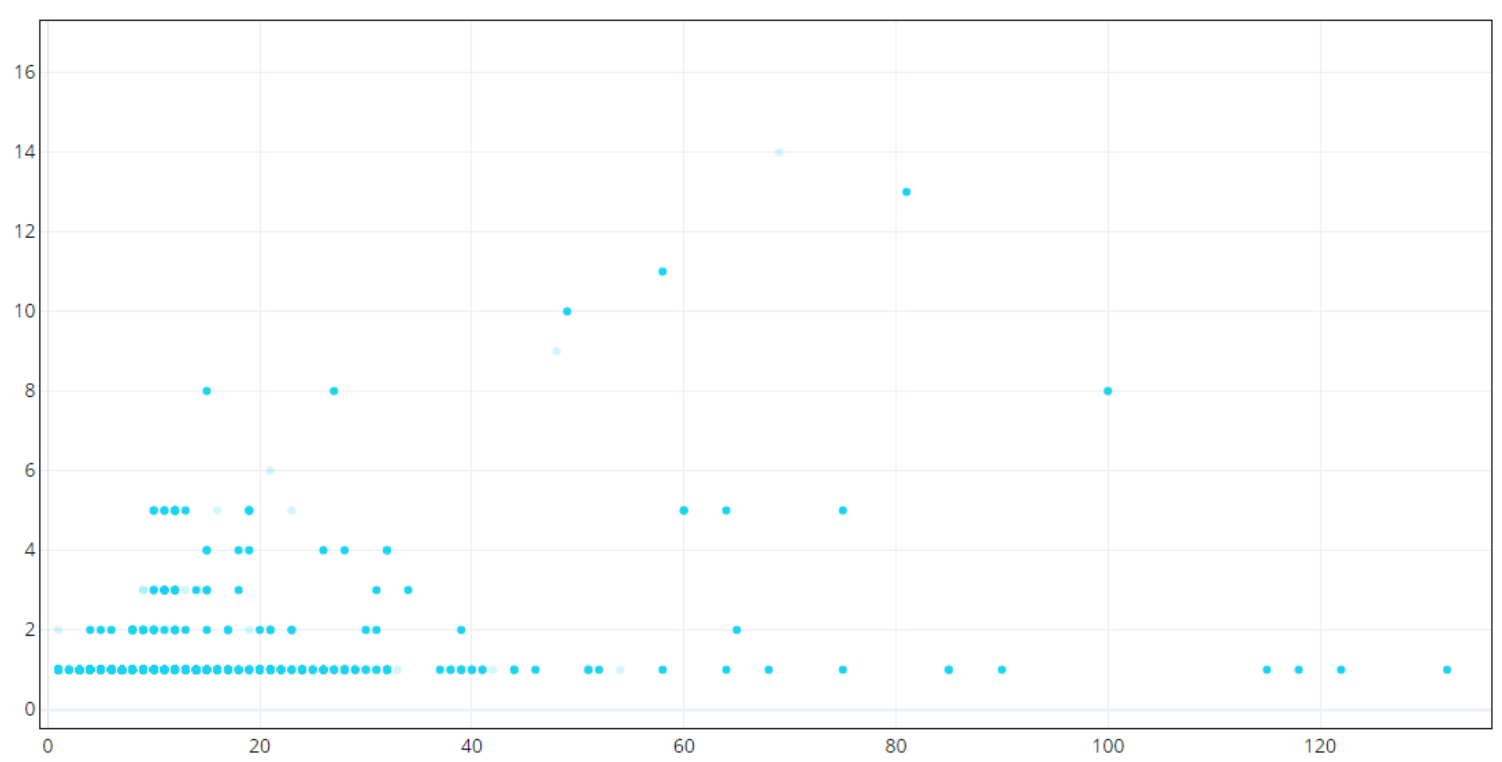

Figure 20. Scatter Plot of LOC (x) vs CC (y) for Amorok (TC), zoomed into the bottom left quadrant.

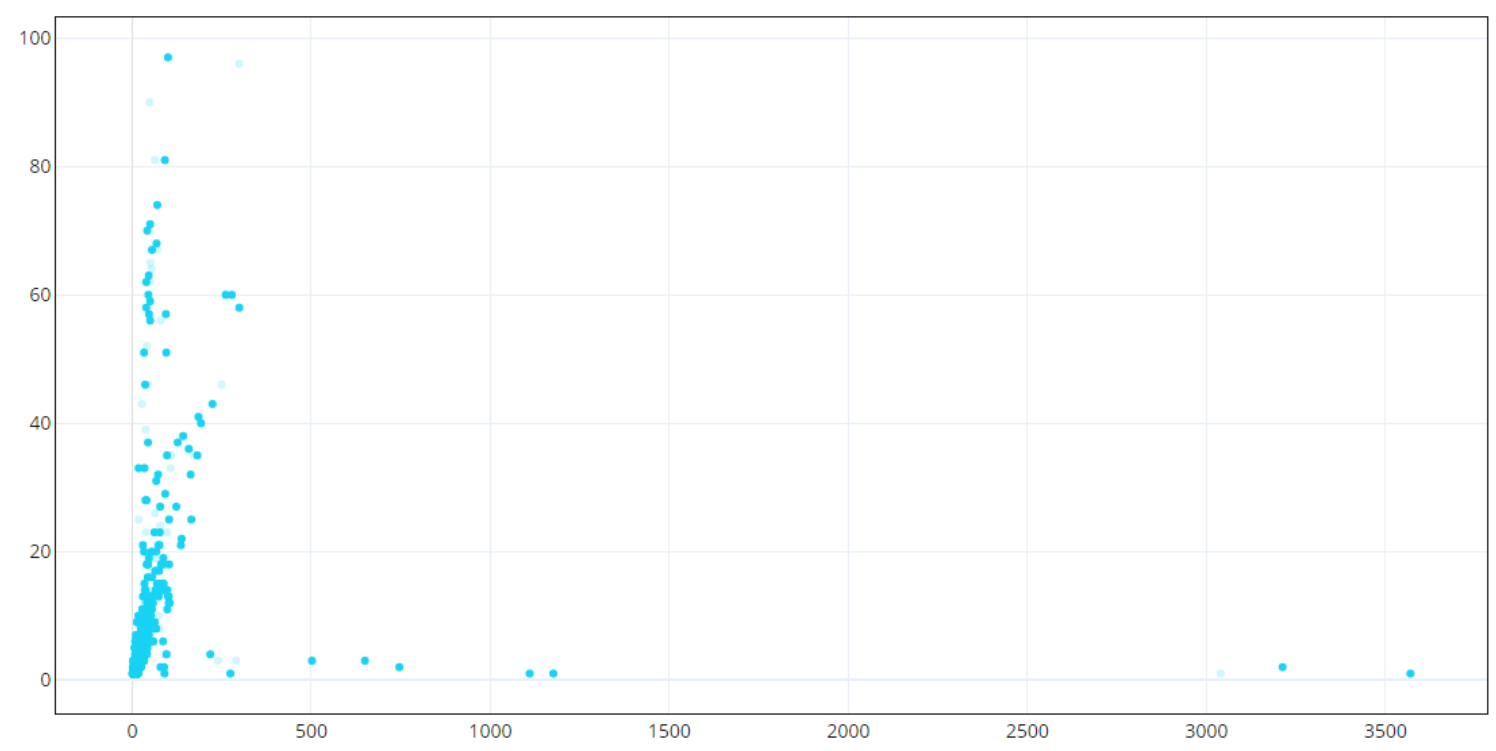

Figure 21. Scatter Plot of LOC (x) vs CC (y) for Curl (TC), zoomed into the bottom left quadrant. 


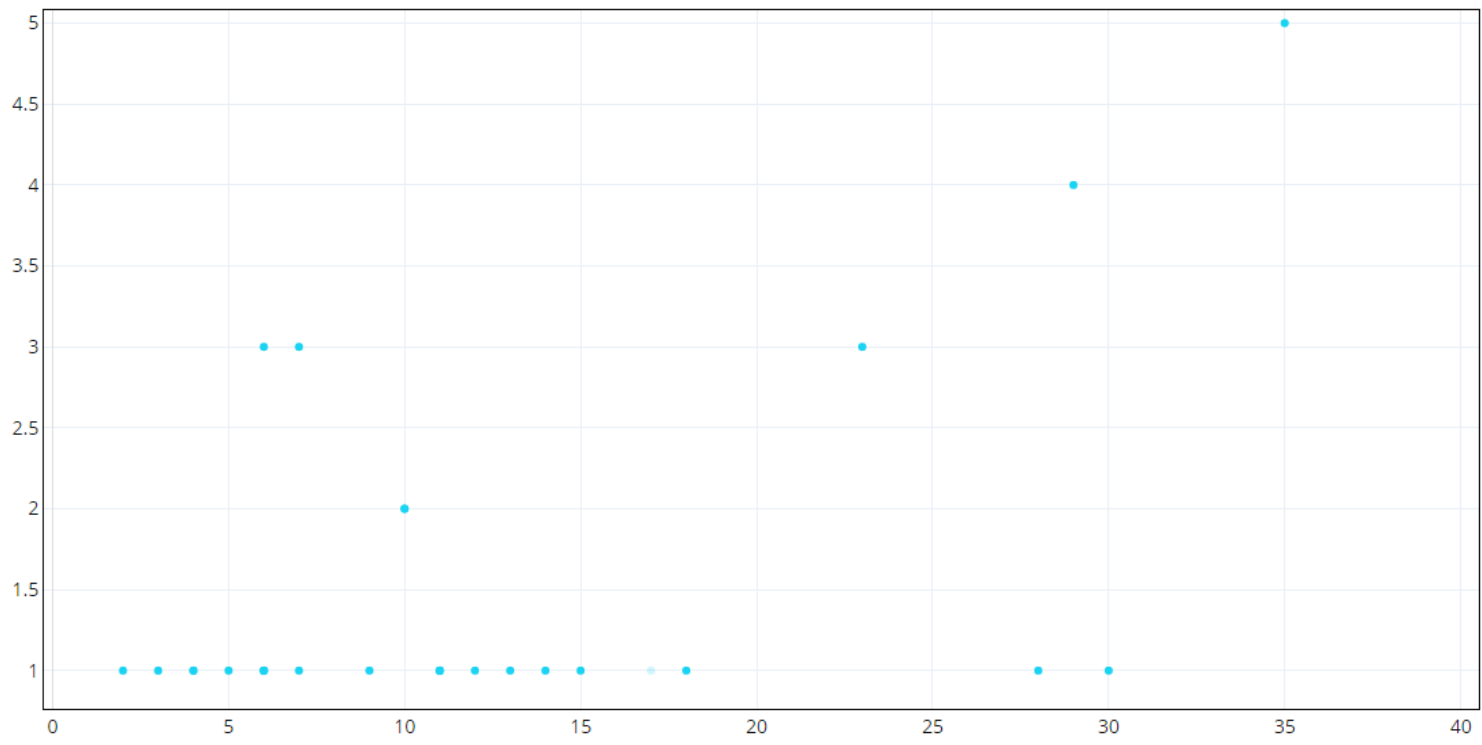

Figure 22. Scatter Plot of LOC (x) vs CC (y) for Curl (TC), zoomed into the bottom left quadrant.

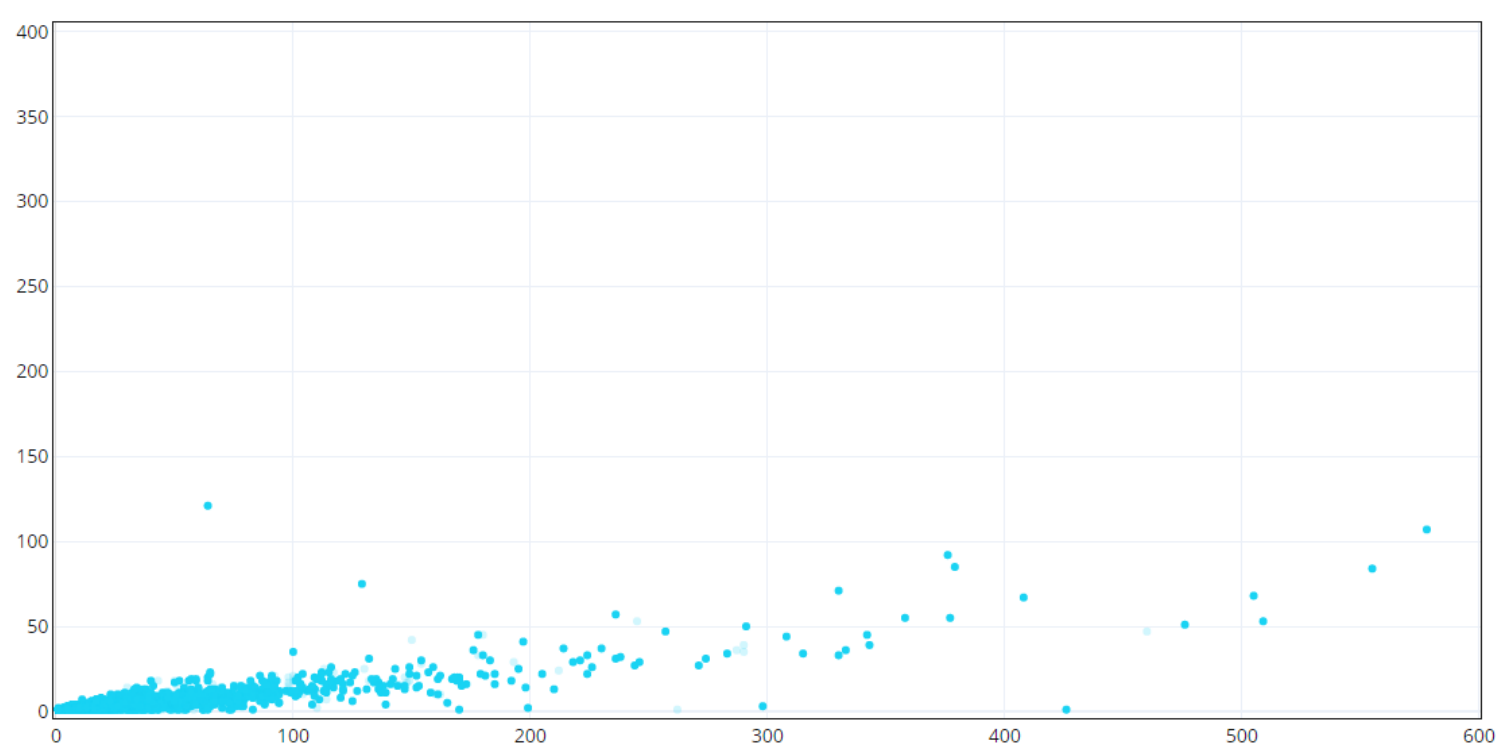

Figure 23. Scatter Plot of LOC (x) vs CC (y) for Handbrake (TC), zoomed into the bottom left quadrant. 


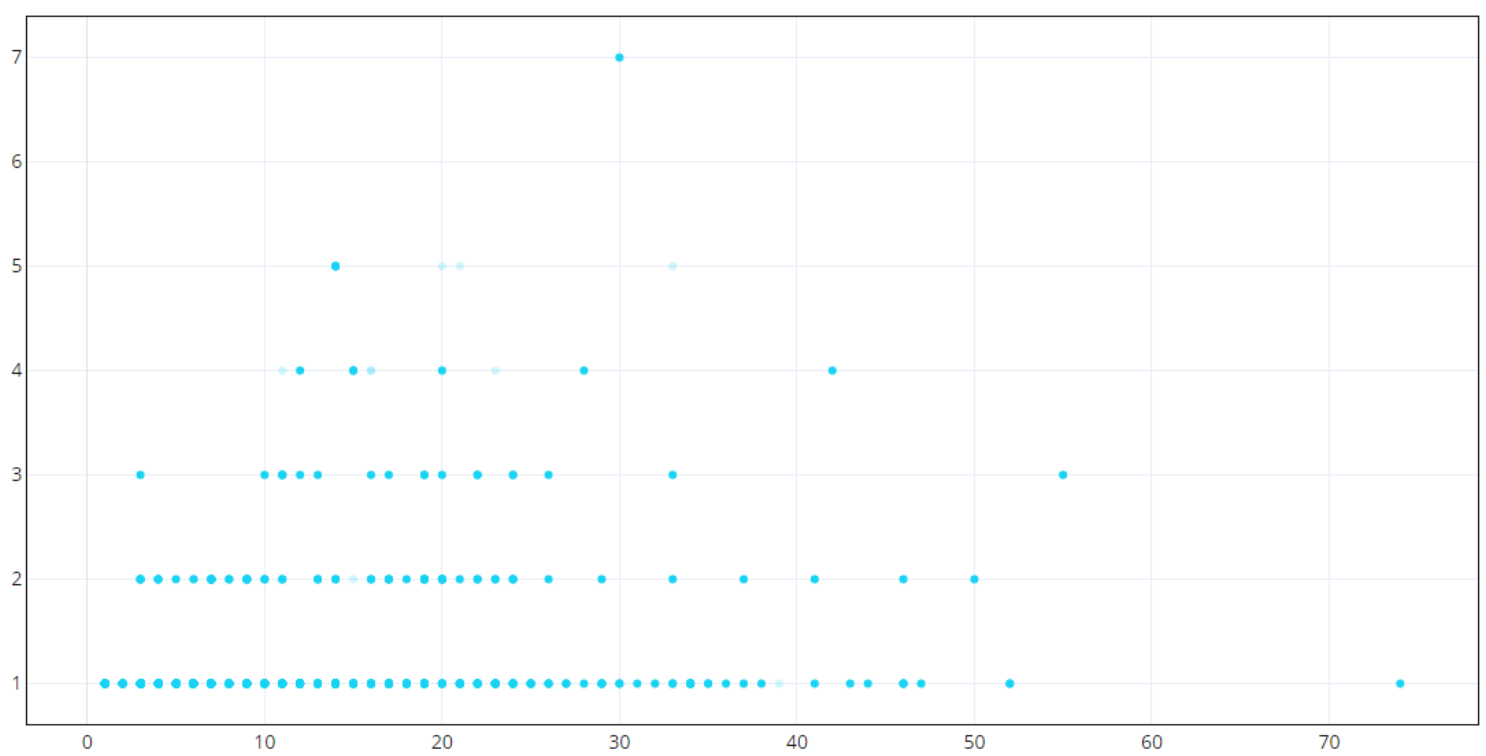

Figure 24. Scatter Plot of LOC (x) vs CC (y) for Hibernate ORM (TC), zoomed into the bottom left quadrant.

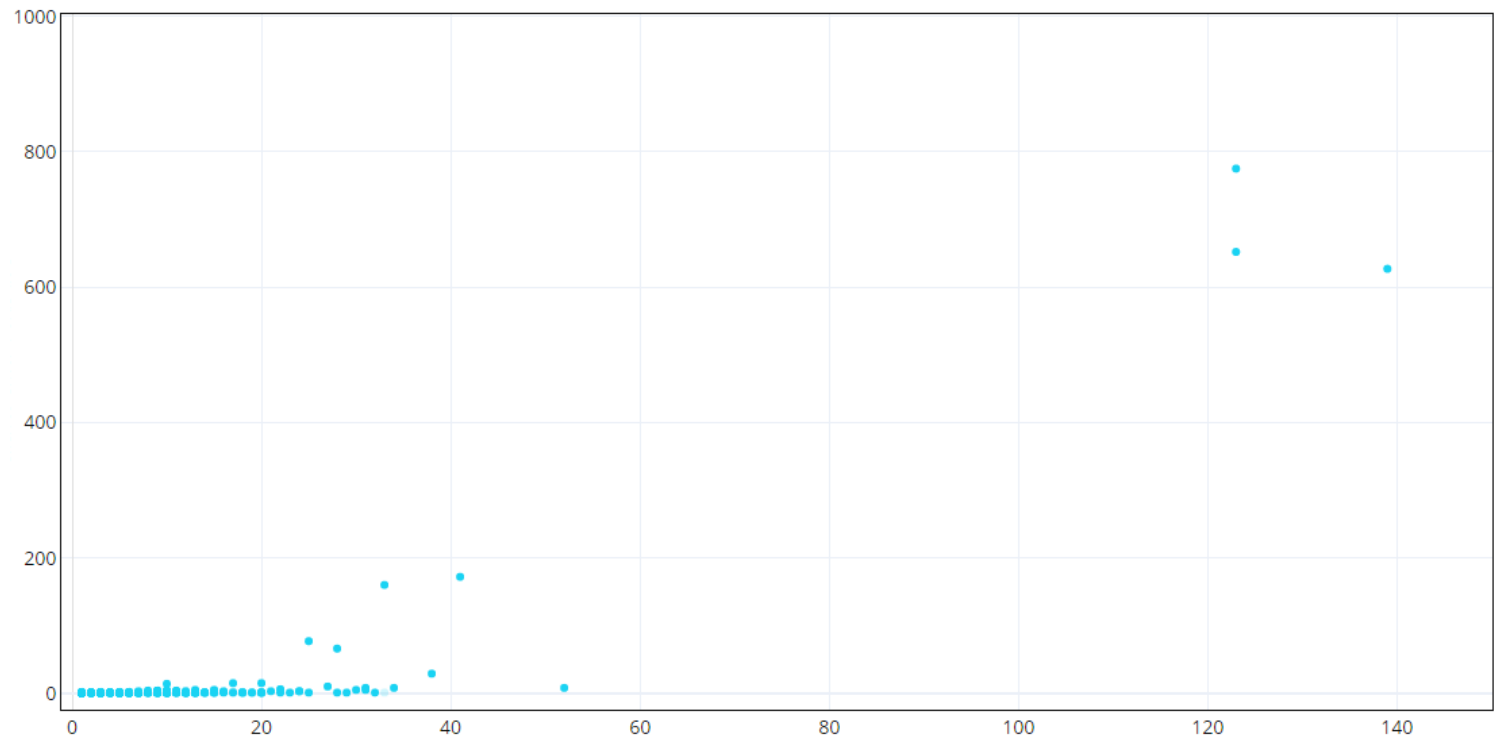

Figure 25. Scatter Plot of LOC (x) vs CC (y) for Hibernate (TC), zoomed into the bottom left quadrant. 


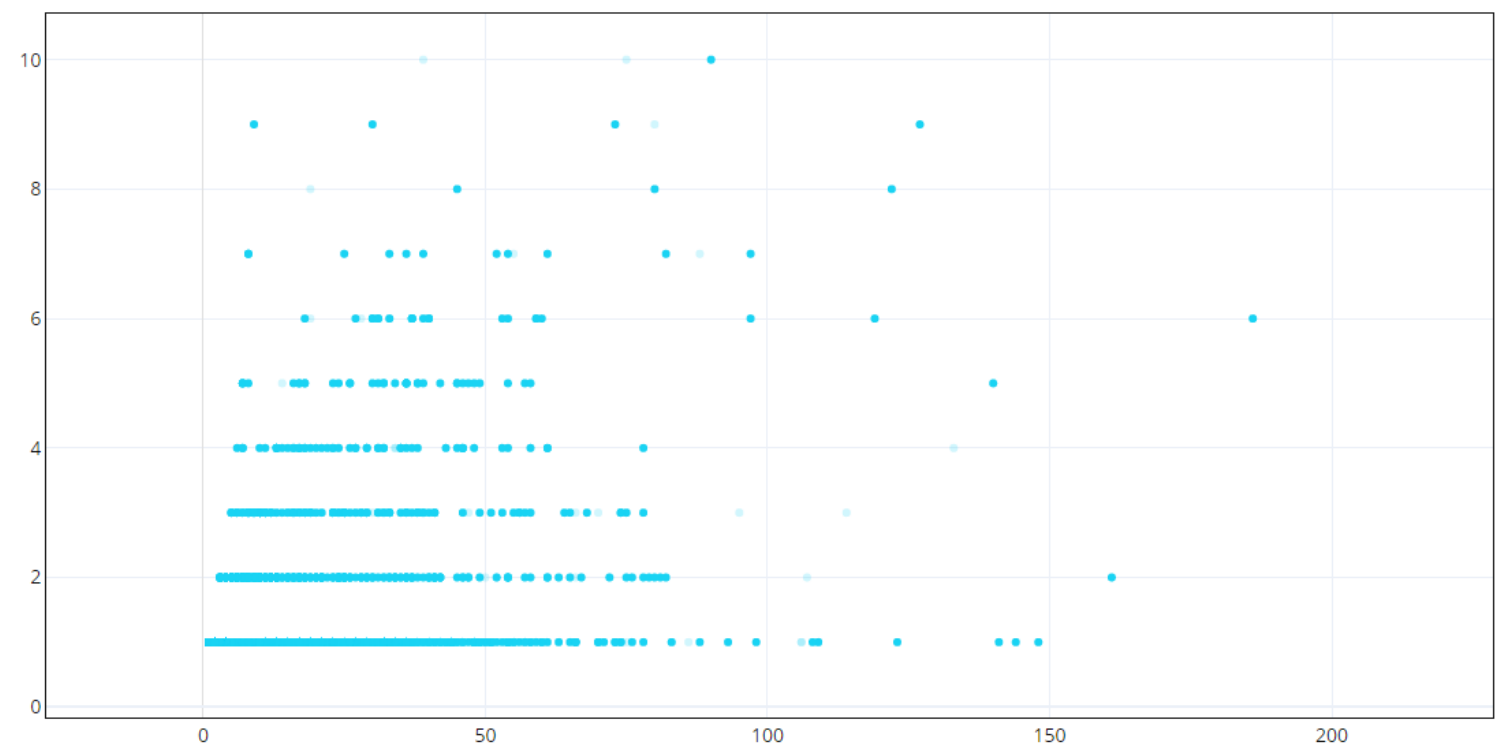

Figure 26. Scatter Plot of LOC (x) vs CC (y) for Notepad ++ (TC), zoomed into the bottom left quadrant.

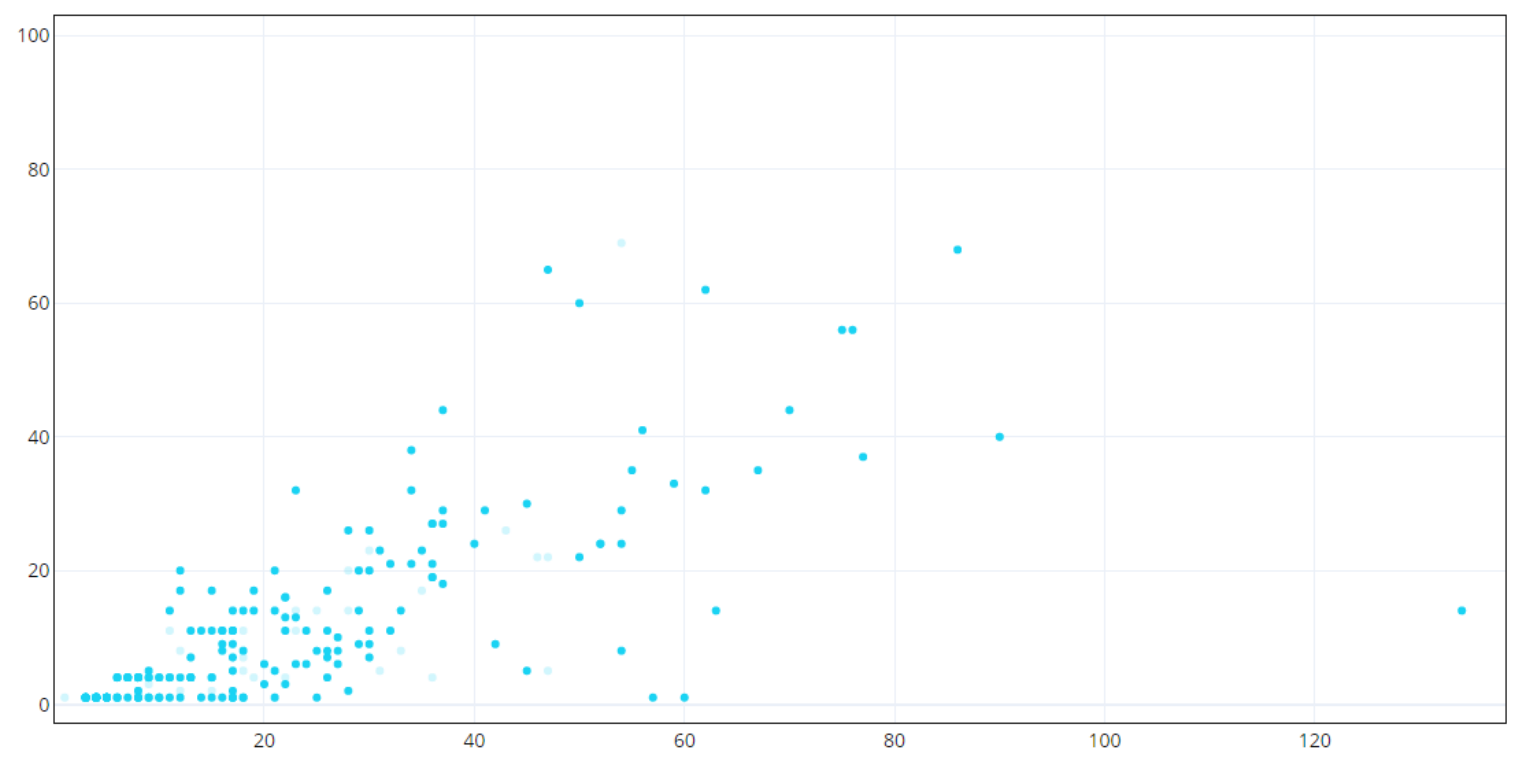

Figure 27. Scatter Plot of LOC (x) vs CC (y) for Onion (TC), zoomed into the bottom left quadrant. 


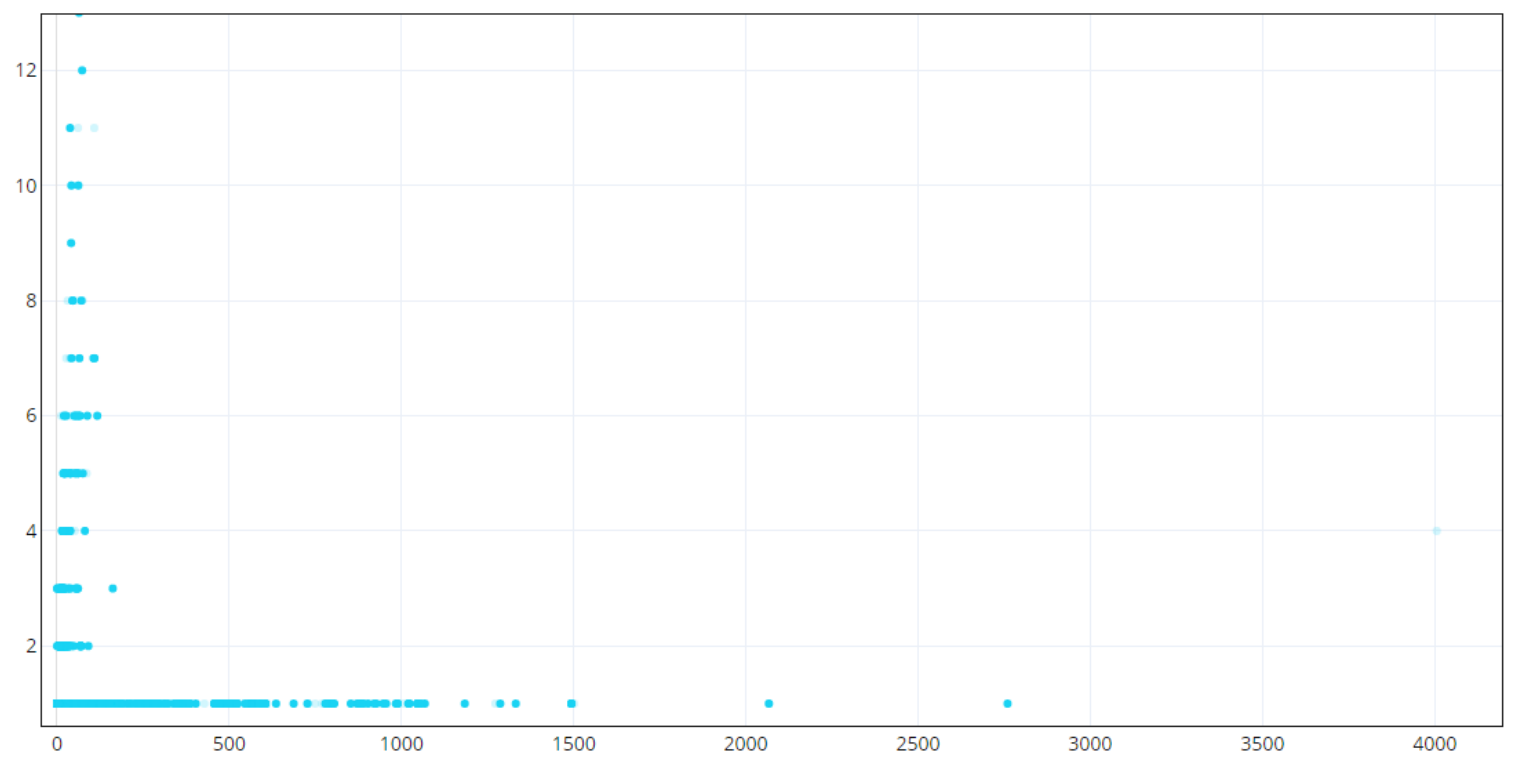

Figure 28. Scatter Plot of LOC (x) vs CC (y) for Open XML (TC), zoomed into the bottom left quadrant.

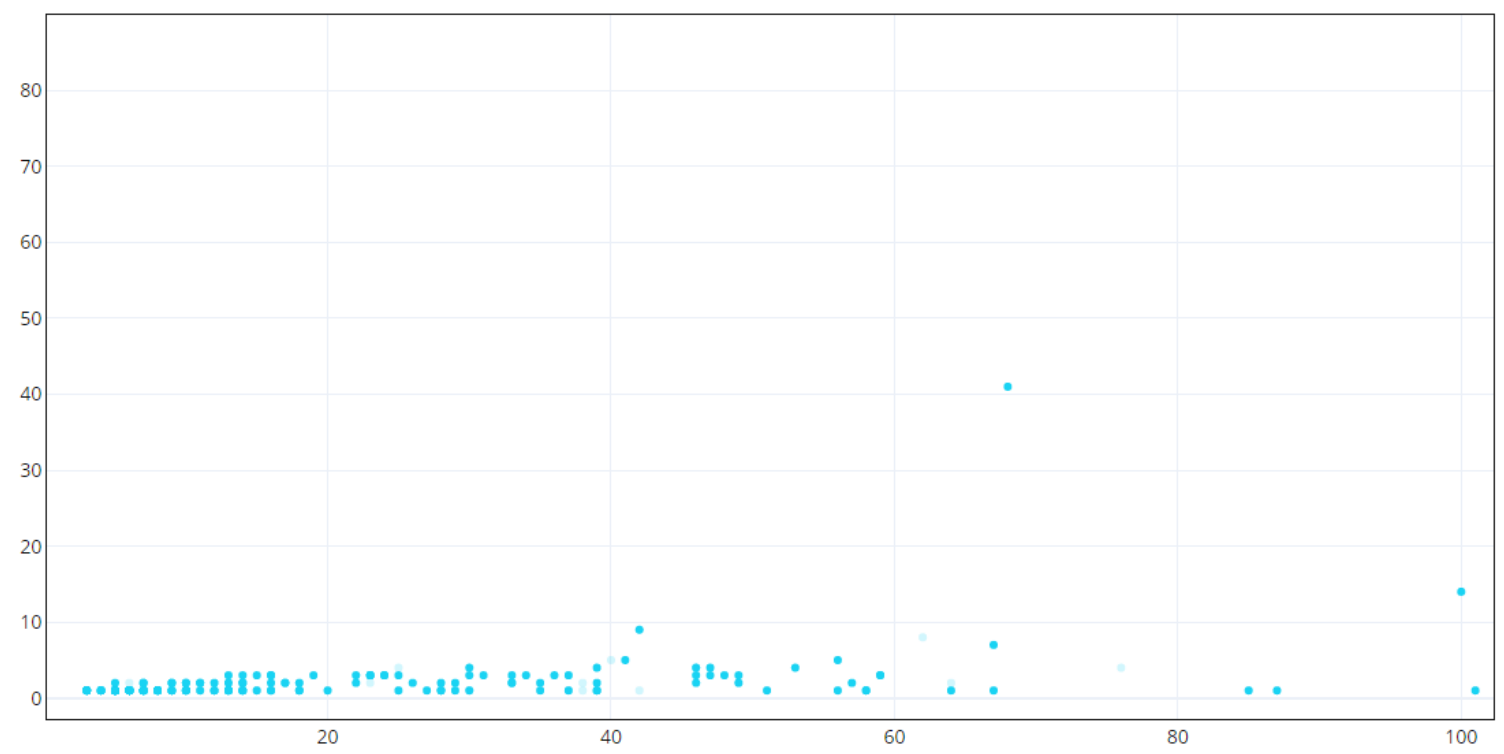

Figure 29. Scatter Plot of LOC (x) vs CC (y) for VLC, zoomed into the bottom left quadrant. 


\section{Appendix C : EXPORTING METRIC VALUES USING SCITOLS VERSION 4.0}

\section{C.1 Analyze Files or Directories Using SciTools Understand.}

Open Understand, and go to File -> New -> Project.

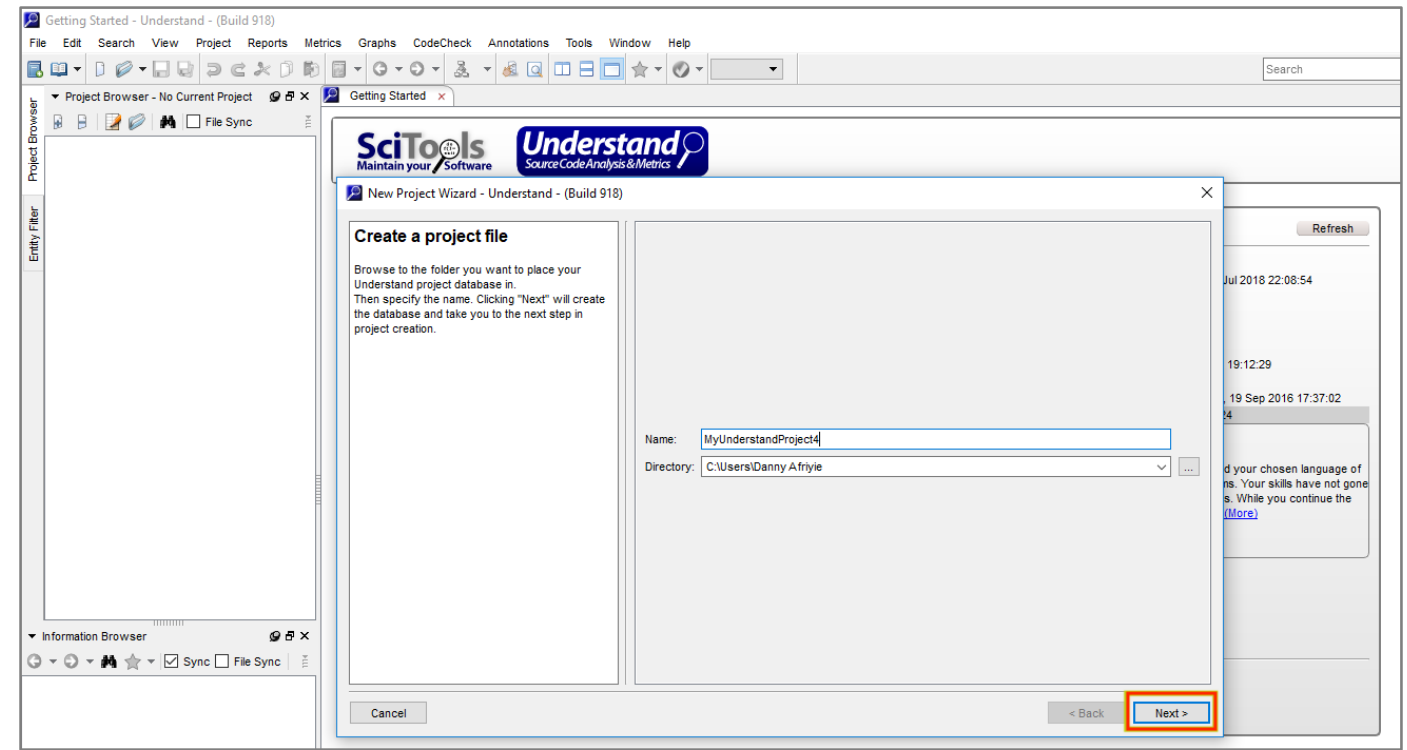

Figure 30. Naming a new Project in Understand

We name the Project with a name such as Project $X X X \_\mathrm{AC}$ or Project $X X X \_$TC depending on whether the code we intend to measure is Application Code (AC) or Test Code (TC). Click on Next.

To maintain privacy and avoid releasing proprietary information, we replace $X X X$ with a name that is meaningful, though not attributable to an organization and record the mapping between the chosen string $X X X$ and the actual details of the product (e.g., product/system/package name and version information) seaprately. This is to enhance data privacy.

We select all Languages to ensure adequate coverage. We do not select VHDL since it is no longer supported by Understand. Tick Fuzzy instead of Strict. Click Next. 
New Project Wizard (C: \Users \Danny Afriyie\MyUnderstandProject4.udb) - Understand - (Build 918)

\section{Languages}

Select the source code language(s) that your project will contain. Later, you can configure options for how each language you select is handled.

C/C++ Fuzzy Analysis: Great for the first pass at most code, since very little setup is required. Uses fuzzy logic to handle incomplete, non-compiling code gracefully and as accurately as possible.

C/C++ Strict Analysis: This option may result in a more accurate analysis, so more setup is required. Include paths and macros will need to be defined during the analysis. Handles $\mathrm{C}++$ templates and overloaded functions better than the fuzzy analyzer. It also will analyze $\mathrm{Objective} \mathrm{C} / \mathrm{C}++, \mathrm{C}+$ +11 and $\mathrm{C}++14$. Include paths and macros need to be defined correctly with this option or Understand will return invalid or incomplete results.

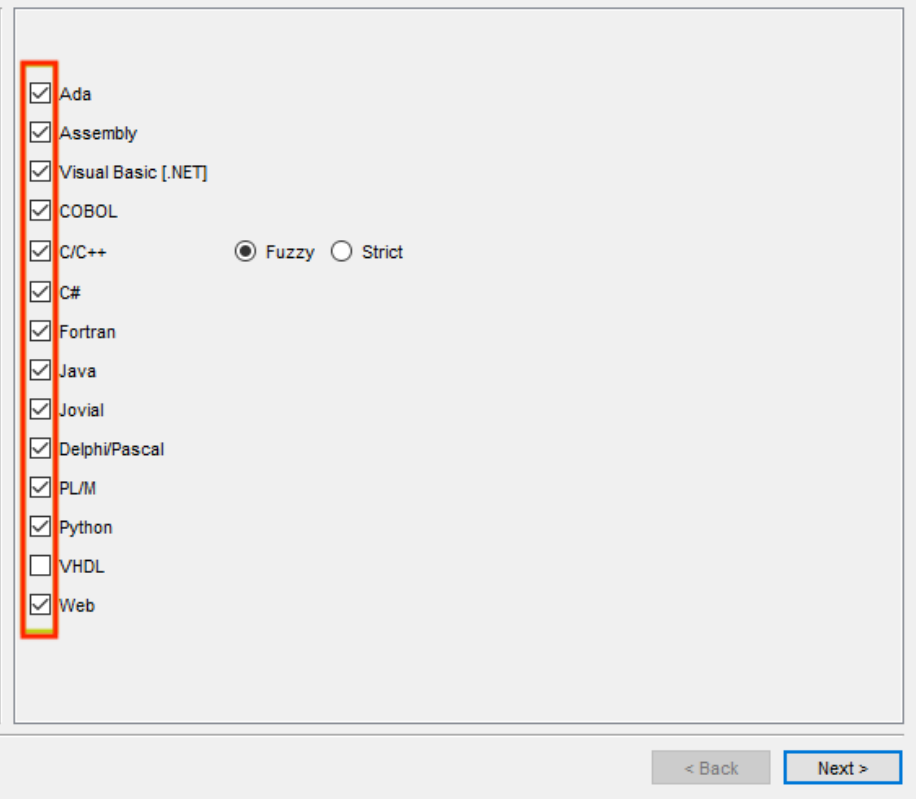

Figure 31. Selecting Source Code Languages Understand should Export

Tick Add source files manually. Click Next.

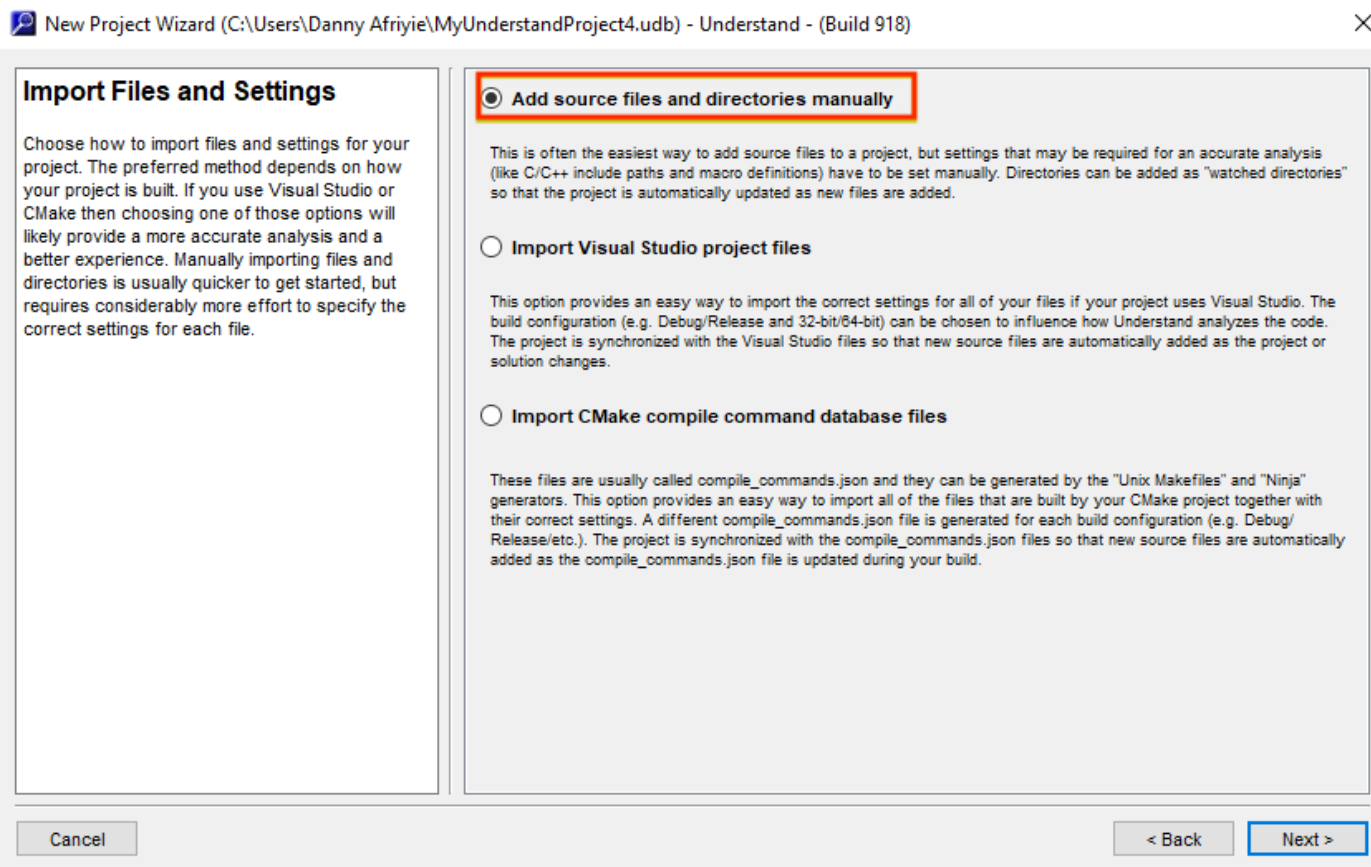

Figure 32. Choosing How to Import Files and Settings

The next window allows us to select a single (source code) file or an entire directory. To select an entire directory, we click on the "Add a Directory". We use the "Add a file" for selecting single files. After selection, we click ok. 

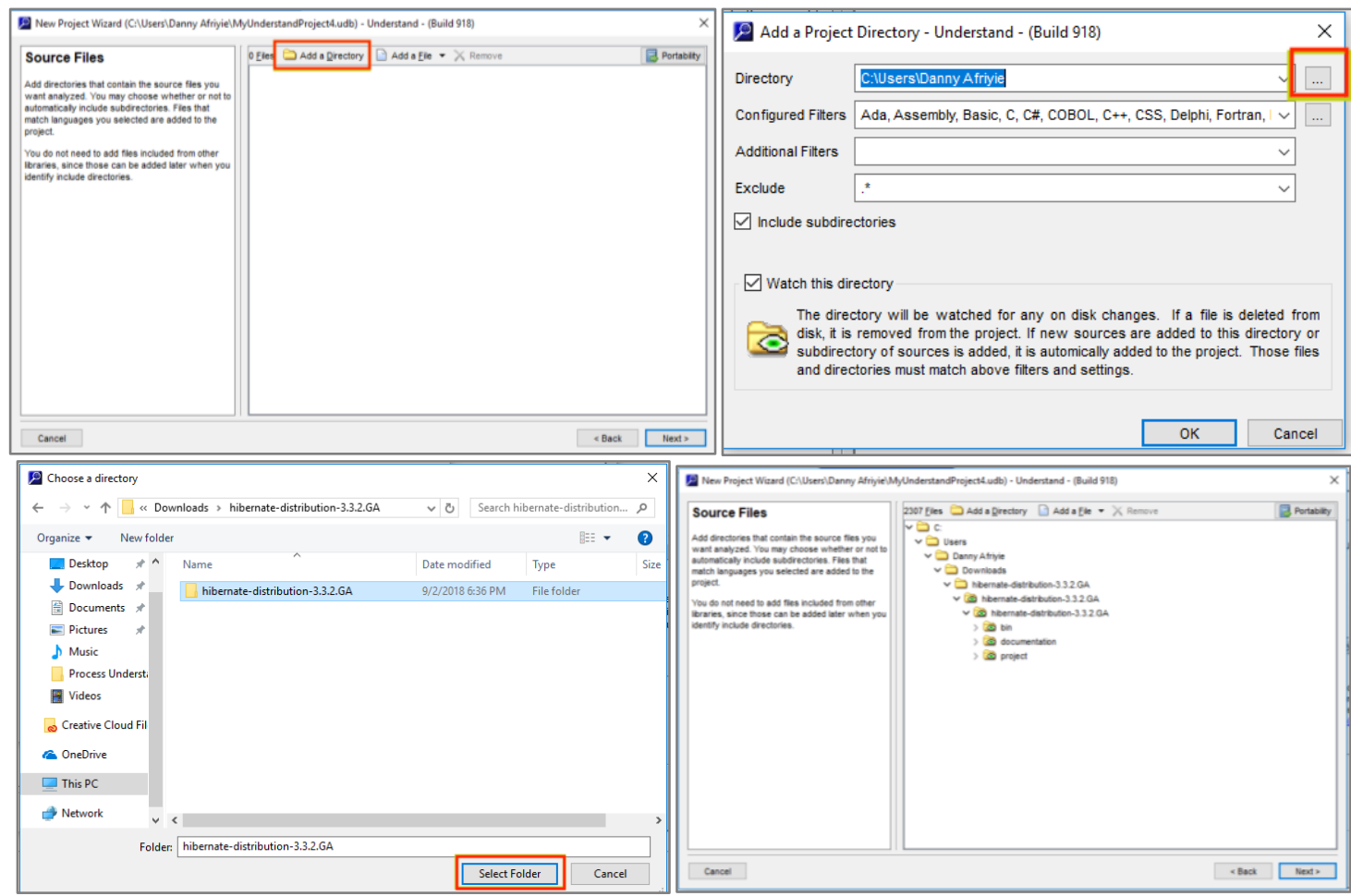

Figure 33. Add Project Directory for Analysis in Understand

Understand will display the file path. We Click Next

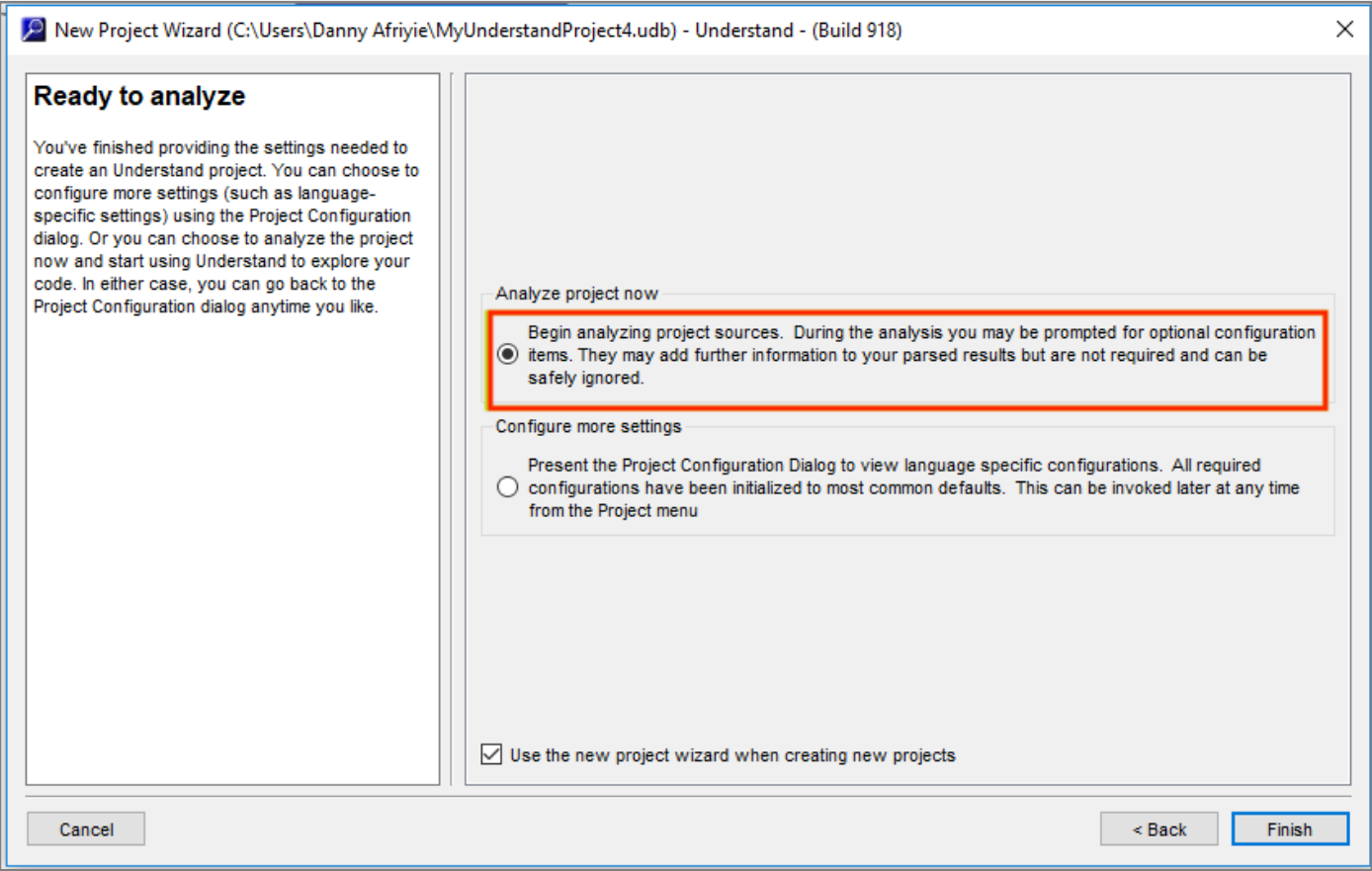

Figure 34. Complete the Project Configuration and Settings for Analysis.

Understand will automatically select begin analyzing project sources. We Click

Finish. 
Understand will start analyzing the code. A progress bar will be shown at the bottom left corner, displaying the percentage of code analyzed.

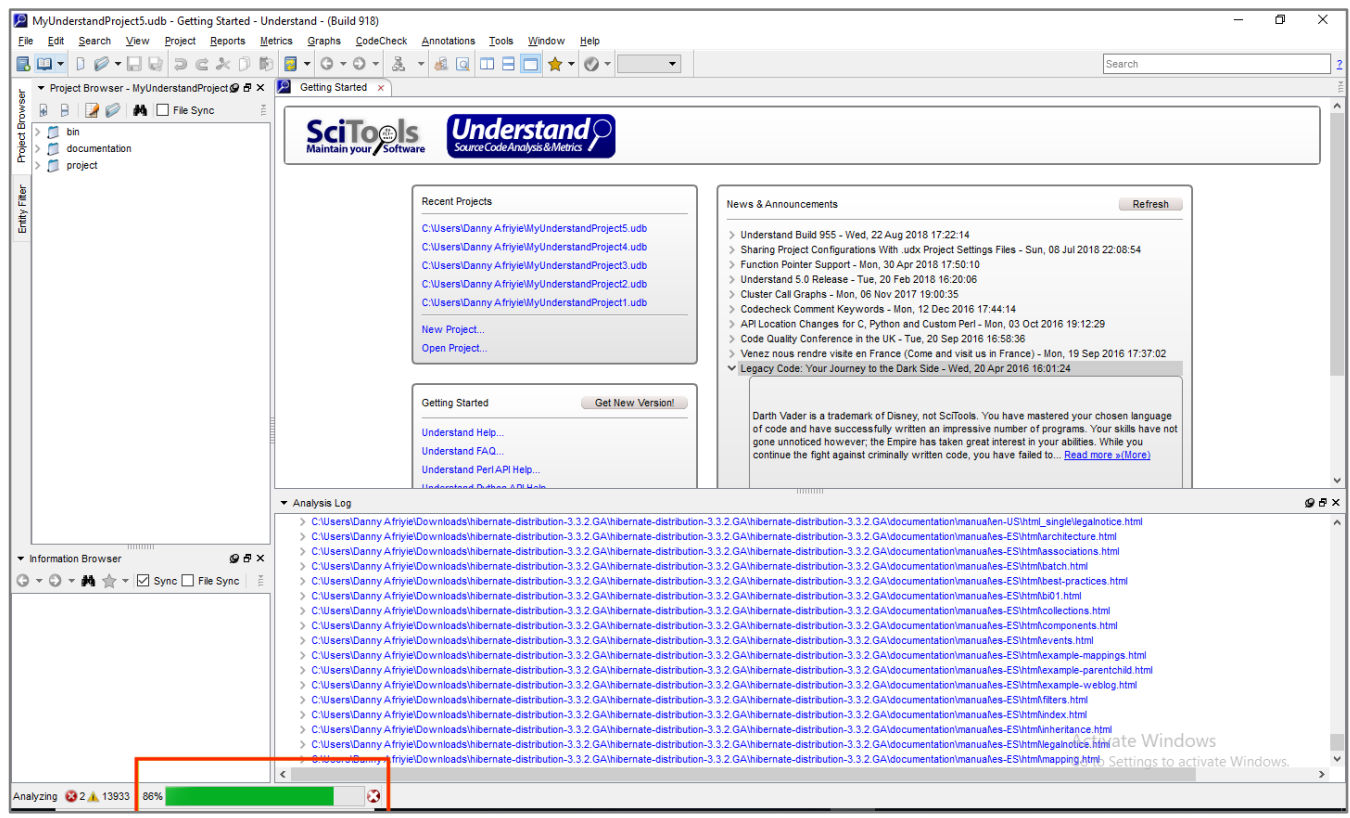

Figure 35. Analysing and Extracting Source Code Information in Understand.

\section{C.2 Exporting Metric Summaries}

In this session, we extract other (simple) metrics, some of which are programming language specific.

\section{C.3 Collecting General Metric Values about Code}

To collect general metric values, such as number of Lines of Code, number of comment lines, we click on Metrics from the Menu Bar, select "Metrics Summary", click on "Copy All" and paste this data into the text editor (Notepad, Word, Pages, etc.) and save the file with a name consistent with the naming convention discussed in Section C.1: for instance Project $X X X \_$AC_Summary and Project $X X X \_$TC_Summary. 


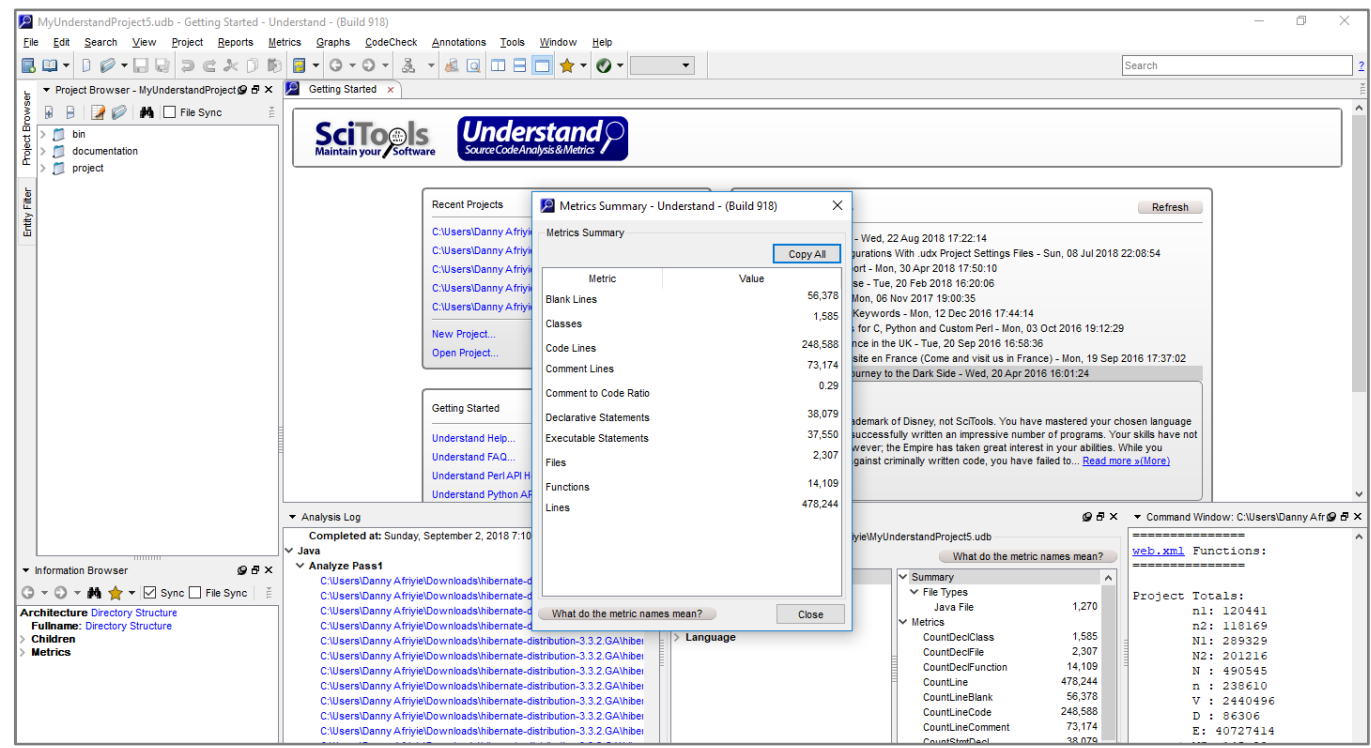

Figure 36. Exporting Metrics Summary for Source Code

\section{C.4 Collecting more detailed Metric values for Different Programming Languages}

To collect more detailed metrics, such as the number of Java classes and methods, we click on "Metrics" from the Menu Bar and select Browse Metrics. A pane with two sections appears at the bottom right corner with two sections: we name the left section $\mathrm{S} 1$ and the right S2 for clarity. The left section (S1) contains a Directory Structure and the right section (S2) lists a metric summary for the files and folders selected in section S1. Drag or enlarge the pane for proper visibility.

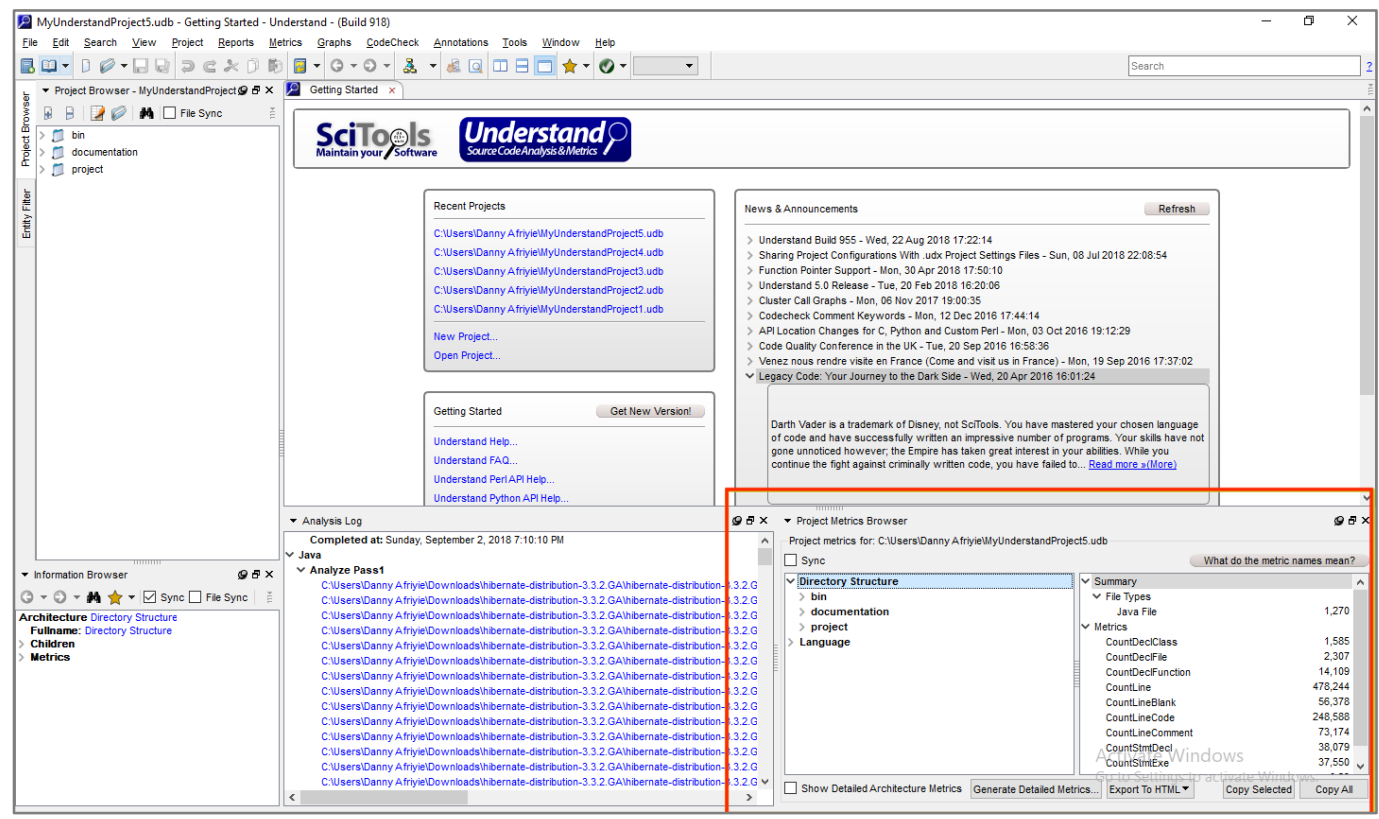


Figure 37. Browse Detailed Metric Summary for Source Code

The tool displays the Directory Structure and a list of the programming languages used. We unfold the list of programming languages and chose a language. After that we right click the programming language, select "Metric Summary", copy-paste the data in a text editor and save according to the previously used naming convention: for instance Project $X X X \_$AC_JavaSummary and Project $X X X \_$TC_JavaSummary if you selected Java. Ignore languages like HTML, CSS, JavaScript.
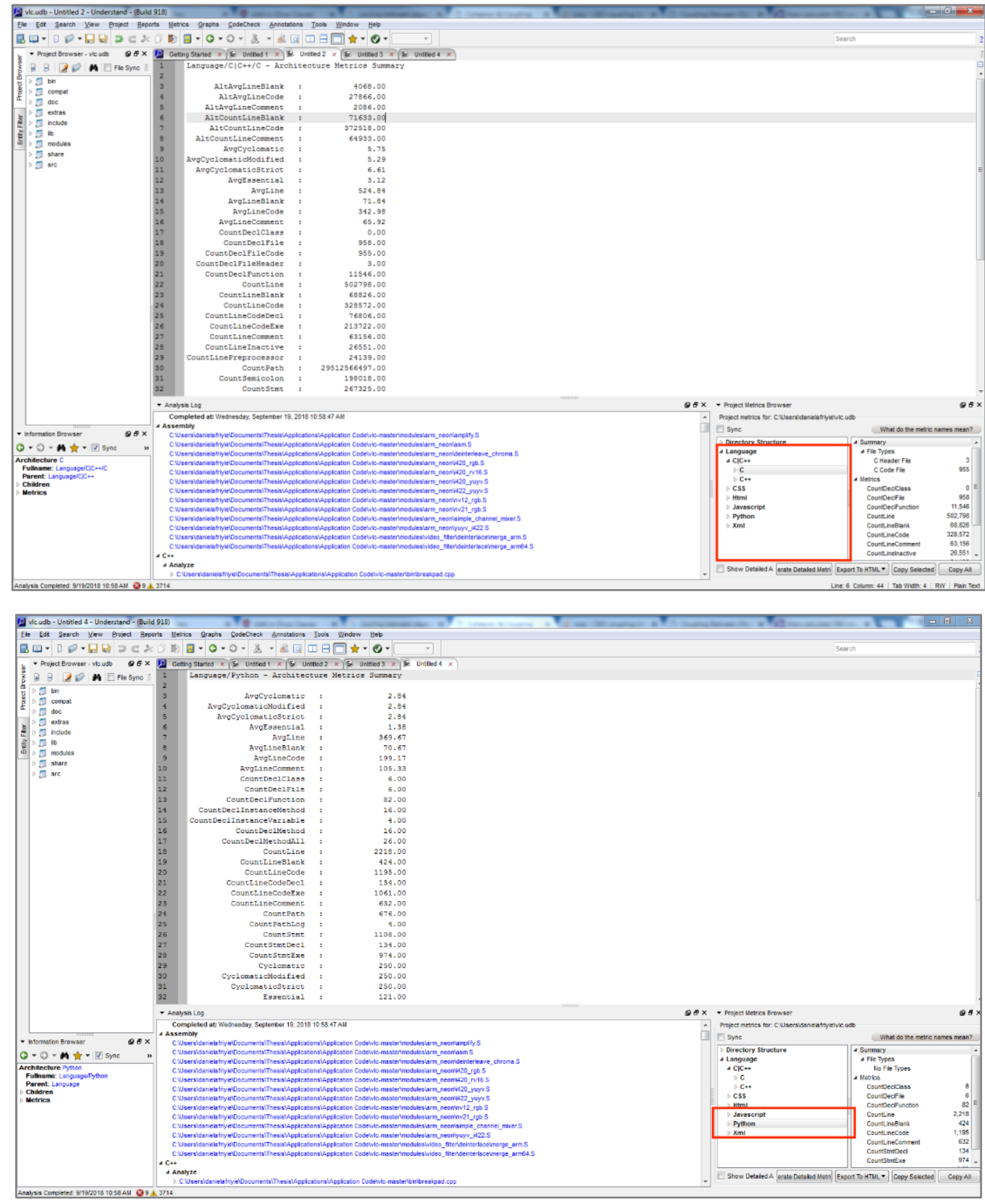

Figure 38. Extracting Detailed Metrics for Specific Programming Languages 


\section{C.5 Ancillary Metrics}

For collecting all detailed metrics in a csv file format, we right click on the Directory Structure on the left section S1 (as described previously) and select Metric Summary. Once it has completed generating the values for the metrics, we select all, copy and paste in a text editor (notepad, word, pages etc.) and save according to the previously used naming convention: for instance Project $X X X \_$AC_AncillaryMetrics for application code and Project $X X X \_$TC_AncillaryMetrics for test code.

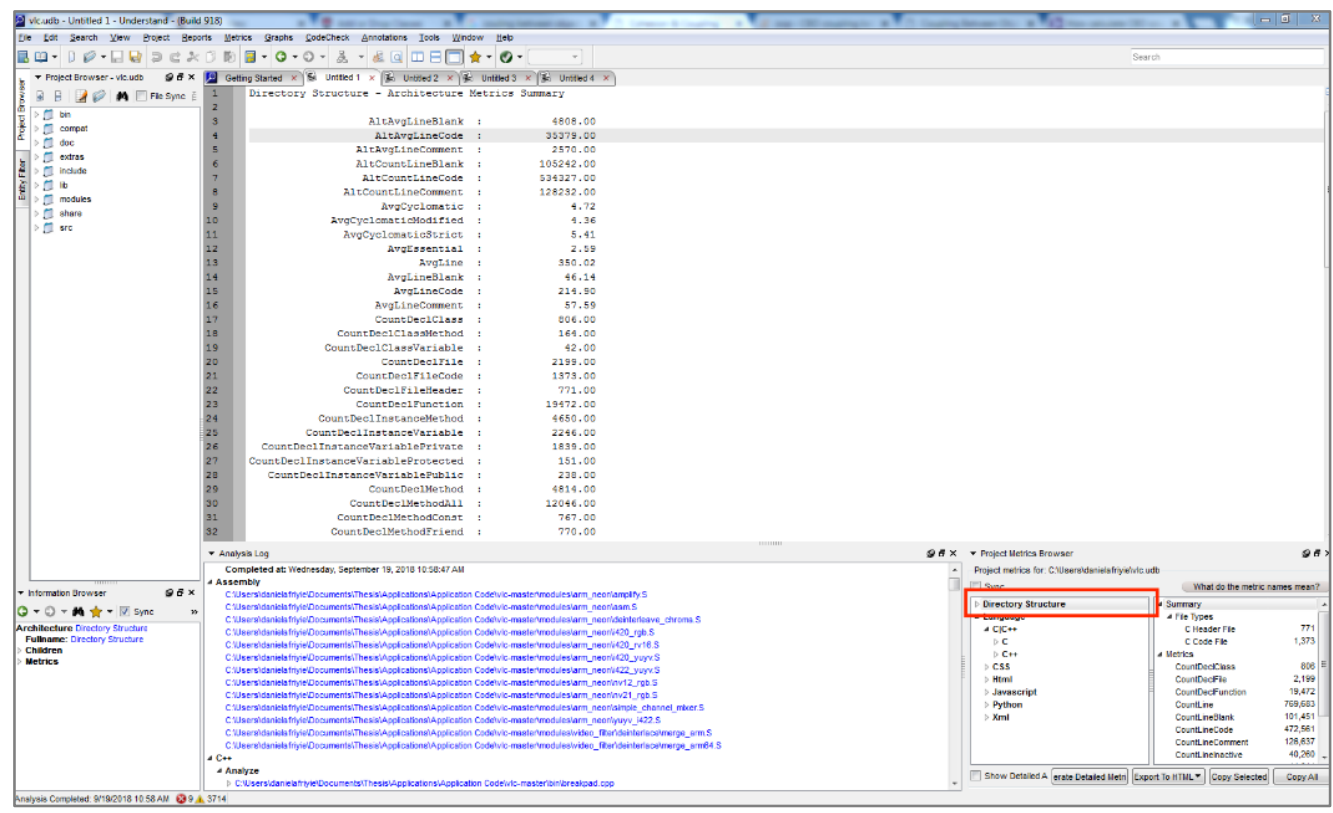

Figure 39. Extract Ancillary Metrics from Understand. 


\section{Appendix D NORMALITY TEST USING SHAPIRO-WILK}

Table 7. Normality Test for Shapiro-Wilk

\begin{tabular}{|c|c|c|c|c|c|}
\hline \multirow[t]{2}{*}{ Application } & \multicolumn{2}{|c|}{ p-value } & \multirow[t]{2}{*}{ Application } & \multicolumn{2}{|c|}{ p-value } \\
\hline & Production Code & Test Code & & \begin{tabular}{|l|} 
Production Code \\
\end{tabular} & Test Code \\
\hline Amorok & $2.2 \mathrm{e}-16$ & $2.2 \mathrm{e}-16$ & JRuby & $2.2 \mathrm{e}-16$ & $2.2 \mathrm{e}-16$ \\
\hline Amule & $2.2 \mathrm{e}-16$ & $2.2 \mathrm{e}-16$ & Keepassx & $2.2 \mathrm{e}-16$ & $2.2 \mathrm{e}-16$ \\
\hline Apache Ant & $2.2 \mathrm{e}-16$ & $2.2 \mathrm{e}-16$ & Light GBM & $2.2 \mathrm{e}-16$ & $8.443 e-15$ \\
\hline Apache Httpd & $2.2 \mathrm{e}-16$ & $3.539 \mathrm{e}-05$ & Mongo & $2.2 \mathrm{e}-16$ & $2.2 \mathrm{e}-16$ \\
\hline Audacity & $2.2 \mathrm{e}-16$ & $4.304 \mathrm{e}-05$ & MPV Player & $2.2 \mathrm{e}-16$ & $2.186 \mathrm{e}-10$ \\
\hline Bitcoin & $2.2 \mathrm{e}-16$ & $2.2 \mathrm{e}-16$ & Musescore & $2.2 \mathrm{e}-16$ & $2.2 \mathrm{e}-16$ \\
\hline Client & $2.2 \mathrm{e}-16$ & $2.2 \mathrm{e}-16$ & Mypaint & $2.2 \mathrm{e}-16$ & $2.379 \mathrm{e}-16$ \\
\hline Curl & $2.2 \mathrm{e}-16$ & $2.2 \mathrm{e}-16$ & NetData & $2.2 \mathrm{e}-16$ & $2.2 \mathrm{e}-16$ \\
\hline Ditaa & $2.2 \mathrm{e}-16$ & $3.12 \mathrm{e}-08$ & NMap & $2.2 \mathrm{e}-16$ & $2.2 \mathrm{e}-16$ \\
\hline Django & $2.2 \mathrm{e}-16$ & $2.2 \mathrm{e}-16$ & Notepad ++ & $2.2 \mathrm{e}-16$ & $2.2 \mathrm{e}-16$ \\
\hline FileZilla & $2.2 \mathrm{e}-16$ & $4.02 \mathrm{e}-09$ & Onion & $2.2 \mathrm{e}-16$ & $2.2 \mathrm{e}-16$ \\
\hline Foundation DB & $2.2 \mathrm{e}-16$ & $2.2 \mathrm{e}-16$ & OpenXML & $2.2 \mathrm{e}-16$ & $2.2 \mathrm{e}-16$ \\
\hline Handbrake & $2.2 \mathrm{e}-16$ & $2.2 \mathrm{e}-16$ & PDF Creator & $2.2 \mathrm{e}-16$ & $2.2 \mathrm{e}-16$ \\
\hline Hibernate ORM & $2.2 \mathrm{e}-16$ & $2.2 \mathrm{e}-16$ & Powershell & $2.2 \mathrm{e}-16$ & $1.21 \mathrm{e}-11$ \\
\hline Hibernate & $2.2 \mathrm{e}-16$ & $2.2 \mathrm{e}-16$ & Rosegarden & $2.2 \mathrm{e}-16$ & $2.2 \mathrm{e}-16$ \\
\hline Industry 1 & $2.2 \mathrm{e}-16$ & $2.2 \mathrm{e}-16$ & Apache Shiro & $2.2 \mathrm{e}-16$ & $2.2 \mathrm{e}-16$ \\
\hline Industry 2 & $1.13 \mathrm{e}-12$ & $2.2 \mathrm{e}-16$ & Apache Sqoop & $2.2 \mathrm{e}-16$ & $2.2 \mathrm{e}-16$ \\
\hline Industry 3 & $7.52 \mathrm{e}-12$ & $2.2 \mathrm{e}-16$ & Squirrel SQL & $2.2 \mathrm{e}-16$ & $2.2 \mathrm{e}-16$ \\
\hline Industry 4 & $2.2 \mathrm{e}-16$ & $2.2 \mathrm{e}-16$ & VLC & $2.2 \mathrm{e}-16$ & $2.2 \mathrm{e}-16$ \\
\hline Industry 5 & $2.2 \mathrm{e}-16$ & 0.02906 & Voldemort & $2.2 \mathrm{e}-16$ & $2.2 \mathrm{e}-16$ \\
\hline Inkscape & $2.2 \mathrm{e}-16$ & $6.232 \mathrm{e}-15$ & VSCode & $2.2 \mathrm{e}-16$ & $2.2 \mathrm{e}-16$ \\
\hline Jfreechart & $2.2 \mathrm{e}-16$ & $2.2 \mathrm{e}-16$ & WorldWind Java & $2.2 \mathrm{e}-16$ & $2.2 \mathrm{e}-16$ \\
\hline Jakarta JMeter & $2.2 \mathrm{e}-16$ & $2.2 \mathrm{e}-16$ & & & \\
\hline
\end{tabular}




\section{Appendix E Collecting data from Understand.}

\section{E.1 Export Metrics Using SciTools Understand Version 4.0}

Here, we describe the process of extracting and anonymizing data from SciTools Understand for the purpose of replication. A detailed description with diagrams is done in Appendix C . One way to simplify the analysis is to separate application code from test code in separate folders and then select either one of the folders or the other (but not both).

The analysis of Application Code was performed separately from the analysis of Test Code. So essentially, the instructions we provide here was executed on Application Code once and on Test Code once. We summarize the extraction process in the next sections with the detailed description in Appendix C .

\section{Analyze Files or Directories Using SciTools Understand.}

- Open Understand, and Go to File -> New -> Project.

- We name the Project with a name such as Project $X X X \_\mathrm{AC}$ or Project $X X X \_\mathrm{TC}$ depending on whether the code we intend to measure is Application Code (AC) or Test Code (TC). Click on Next.

- We select all Languages to ensure adequate coverage. We do not select VHDL since it is no longer supported by Understand. Tick Fuzzy instead of Strict. Click Next.

- The next window allows us to select a single (source code) file or an entire directory. To select an entire directory, we click on the "Add a Directory". We use the "Add a file" for selecting single files. After selection, we click ok. Understand will display the file path. We Click Next

- Understand will automatically select begin analyzing project sources. We Click Finish.

- Understand will start analyzing the code. A progress bar will be shown at the bottom left corner, displaying the percentage of code analyzed.

\section{E.2 Basic Metrics with Methods Calls and Halstead using A Script}

\section{Exporting Metrics Data using Understand}


In our study we wrote scripts using the Application Programming Interfaces (APIs) of Understand to extract the preferred metrics suitable for our study rather than the generic ones provided through the interface of the application (Understand). Now that Understand has been instructed to process code and generate its own representations of code data, we ask Understand to process that data to produce metrics data. On the Menu bar Click on Tools. We select Run. A pop-up Window will appear.

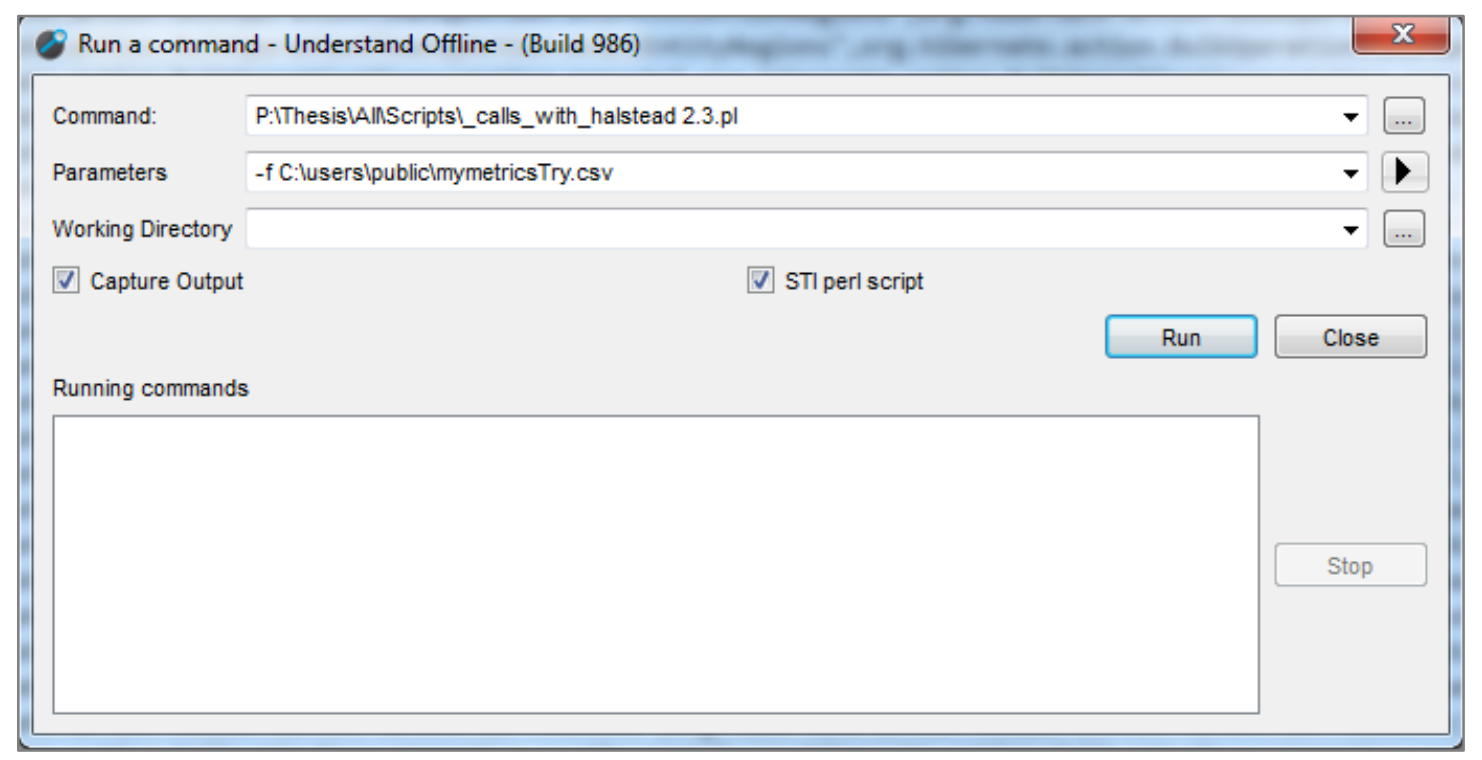

Figure 40. Specifying file paths to Run a Script Using Command in Understand

Understand requires two inputs in order to proceed:

In the Command field, we browse into the location folder to where the script file “_calls_with_halstead 2.3.pl" is saved.

In the parameter field, we specify the where Understand saves the analysis results (the metrics values). We use the same naming convention as in Appendix C Section C.2. Here, a careful attention needs to be given the format of this input parameter. The parameter must start with "-f ", followed by the fully specified path to the location on disk where Understand is to save the results. An example input is: "-f C:lusers\...ProjectXXX.csv".

The third (optional) input Working Directory field is kept empty since Understand automatically recognizes it by default.

We click Run. The run will grey out the UI of Understand, meaning it is extracting data in the background. 
For our purpose we will use two scripts, one written in Perl and the other written in Visual Basic:

- calls_with_halstead 2.3.pl for exporting all metrics.

- Anonymizing Script 1.3 for anonymizing exported data.

The call_with_halstead 2.3 code is written in Pearl using Understand APIs to write a custom script for exporting preferred metrics values with a generic code structural template (or base) from SciTools Understand.

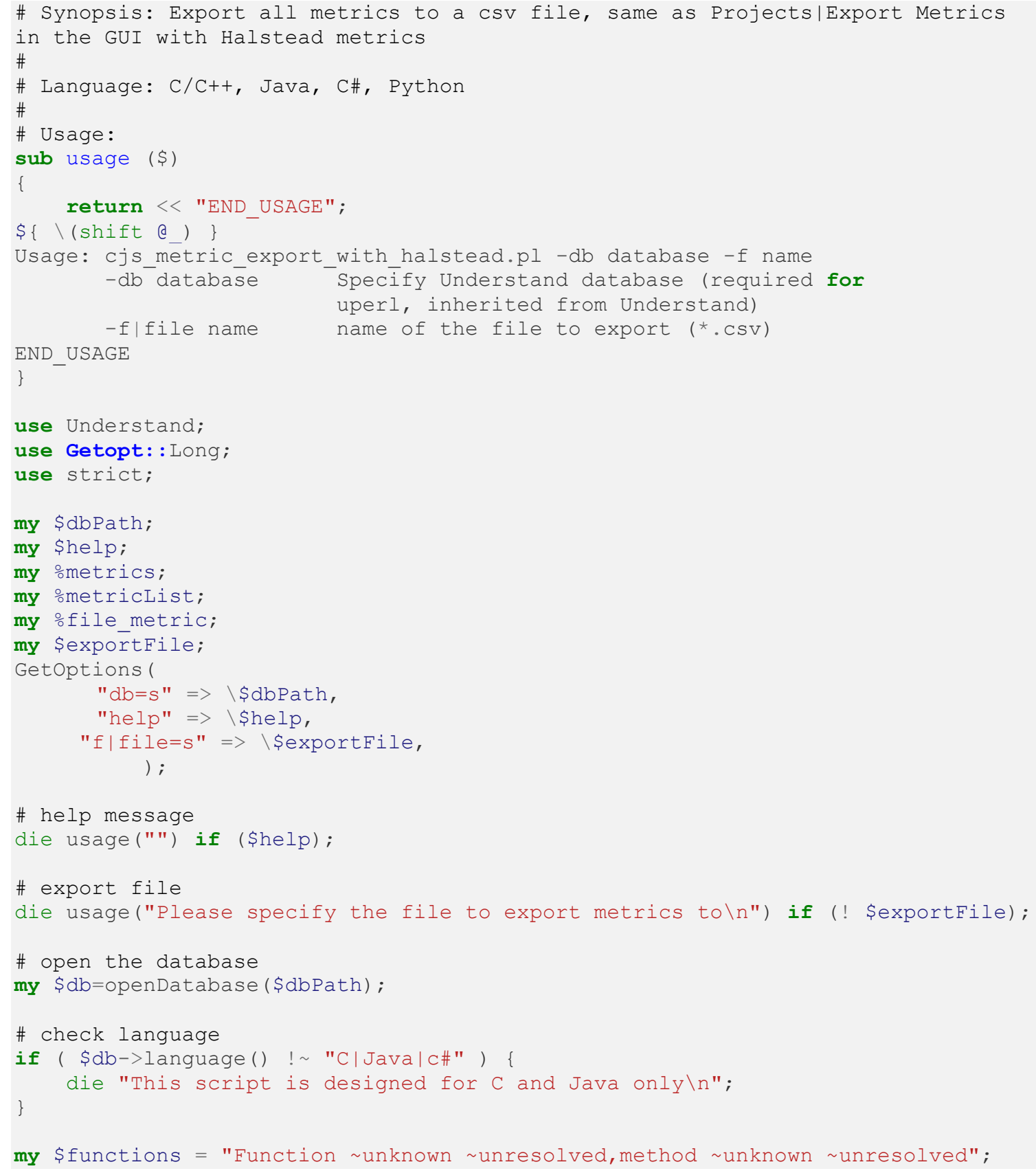




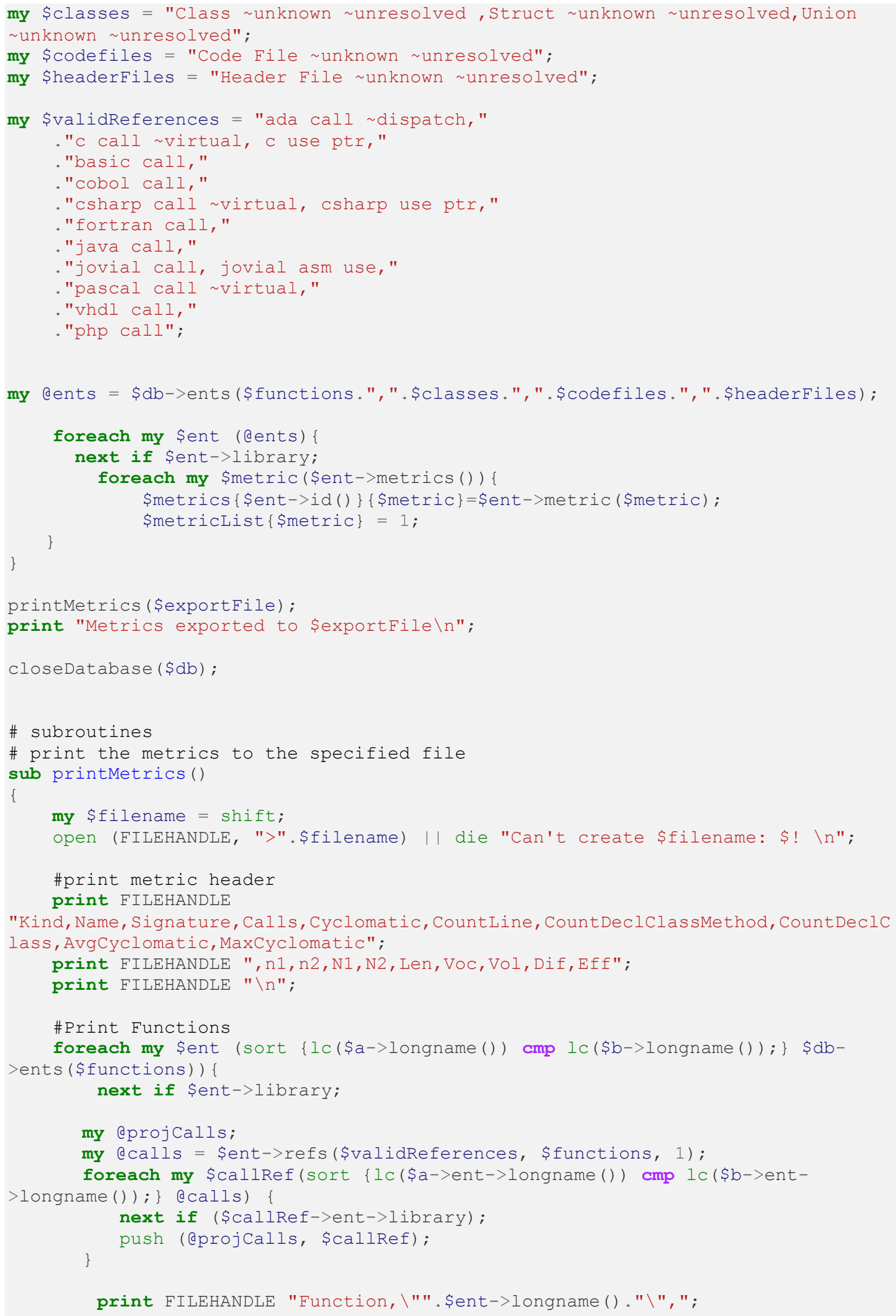




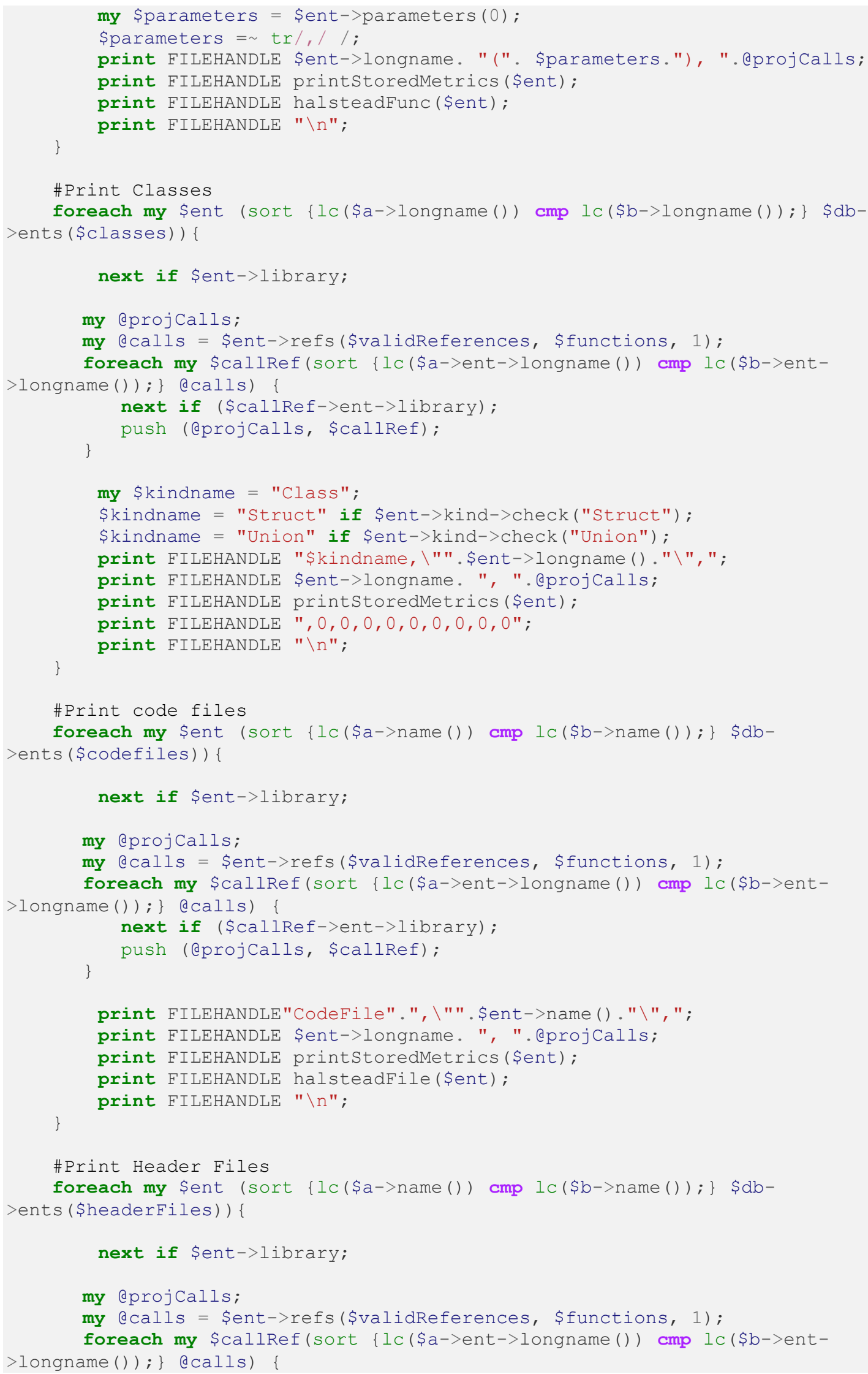




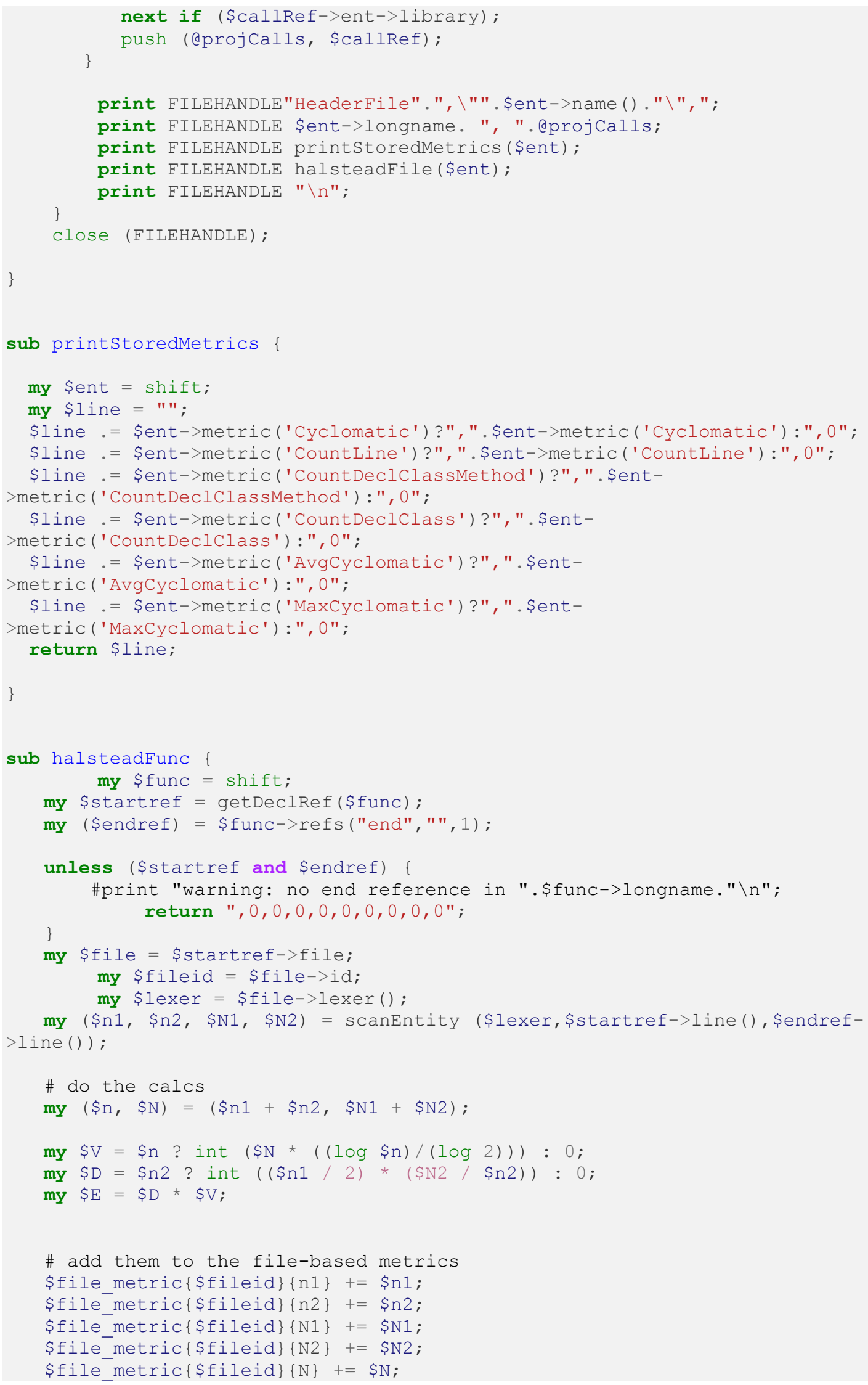




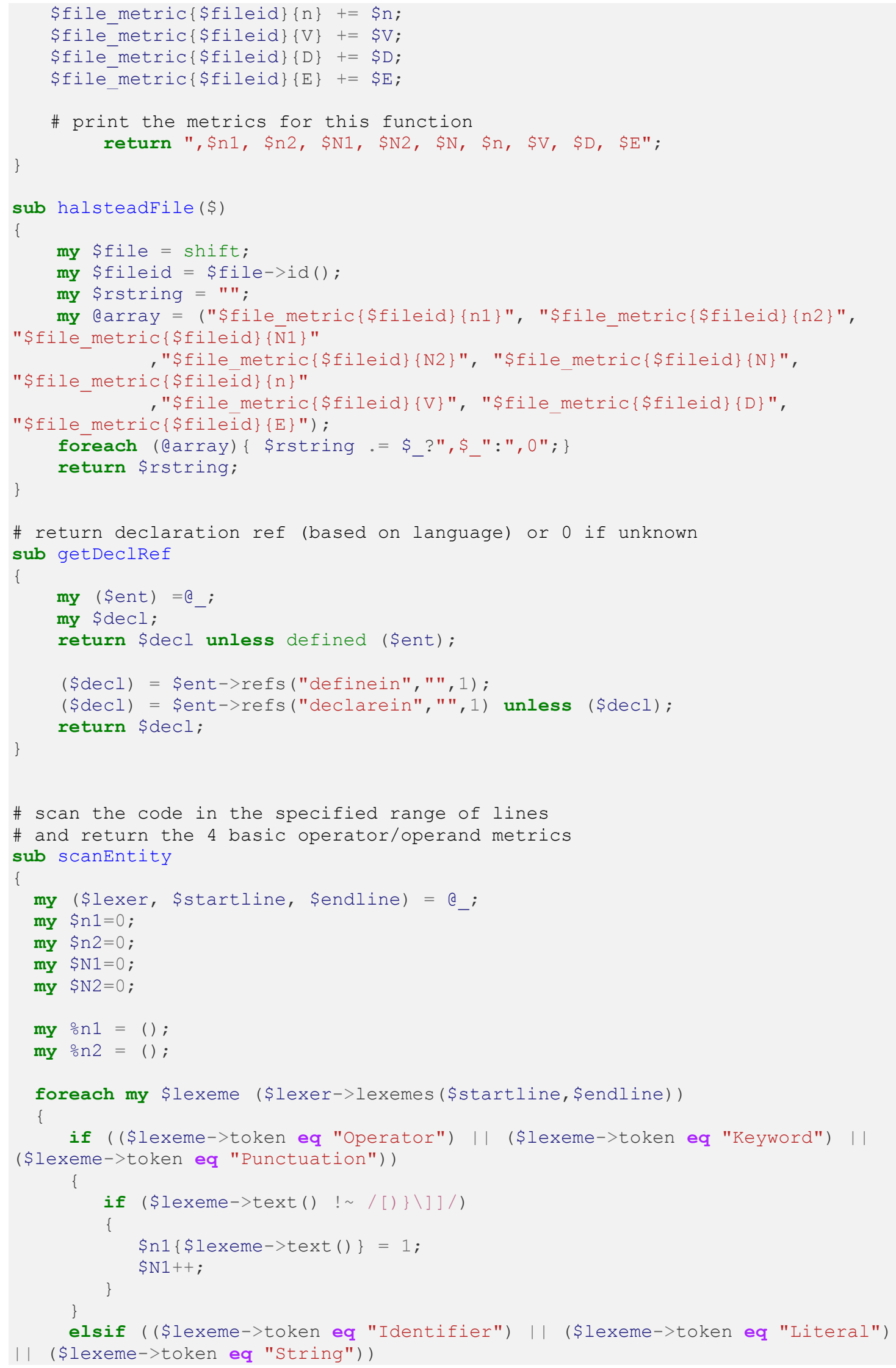




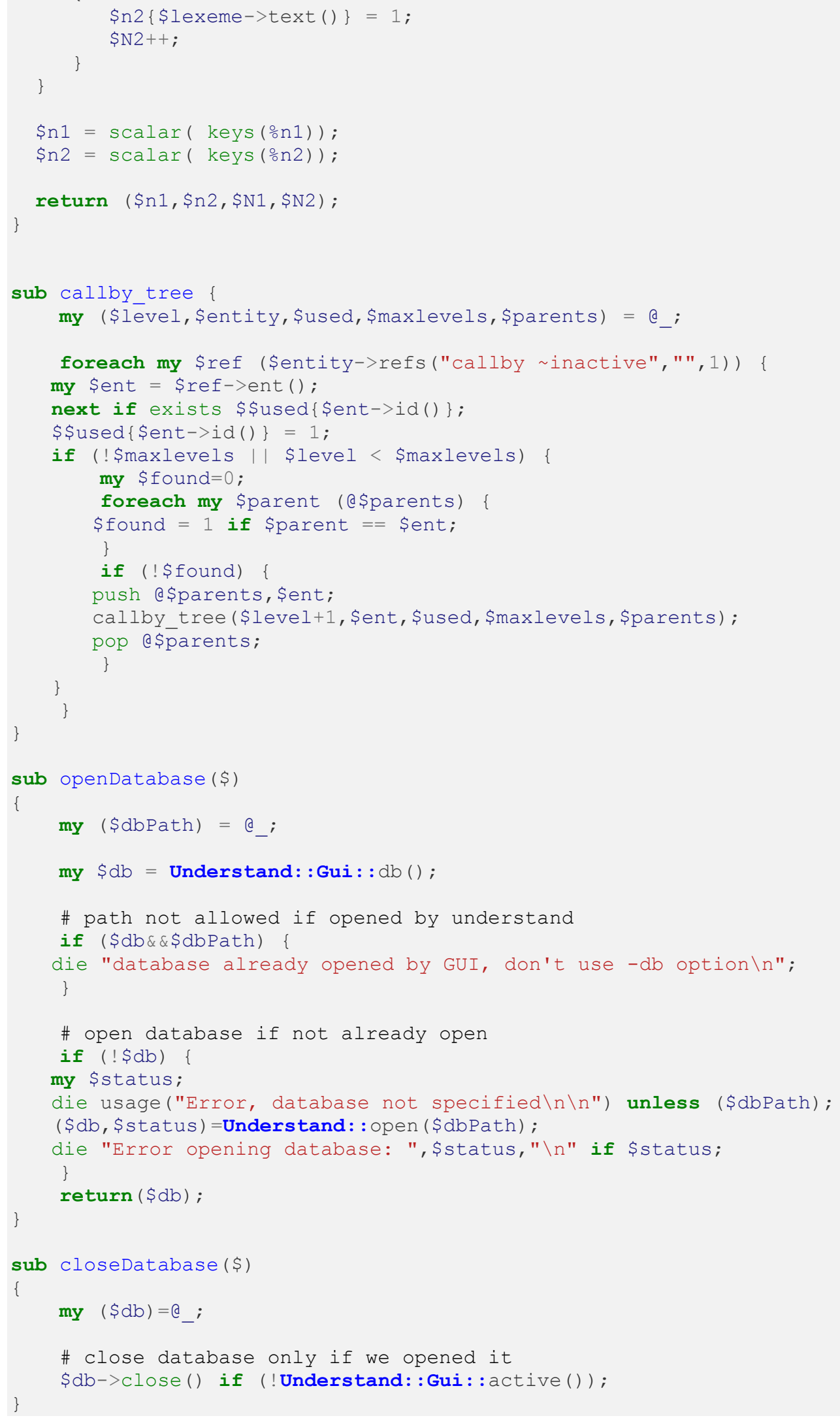




\section{Anonymizing Data in an Excel File}

Understand exports explicit details about the code including file names, source directories, paths, and source code information revealing sensitive information about the code and the company we are extracting from. When exporting data from our Industry partners, our aim was to write a script that anonymizes all these information to avoid any ability to map a particular extract to an organization. The anonymizing process goes as follows: we Open the csv files in Microsoft Excel and anonymize the data using the Anonymizing Script 1.3. This is done either importing it into Macros or copying and pasting the code into the Macros Editor. We Save the files after the anonymization is complete. To use Marcos in Excel this is one of the many procedures we used.

- Open File. Go to Options. select Customize Ribbon. On the right section, Under the Customize the Ribbon, select Main Tabs, and check Developer in the checkbox. Click OK.

- Once developer appears on the Main Tab, select Developer and click on Visual Basic. Under VBA Project, double click on the name of the project as shown in the left pane to open the text editor. Copy and paste the code in Anonymizing Script 1.3 and Run (Play icon).

- Once this is complete, Save the new anonymized data using the preset naming convention

The anonymizing script 1.3 follows as:

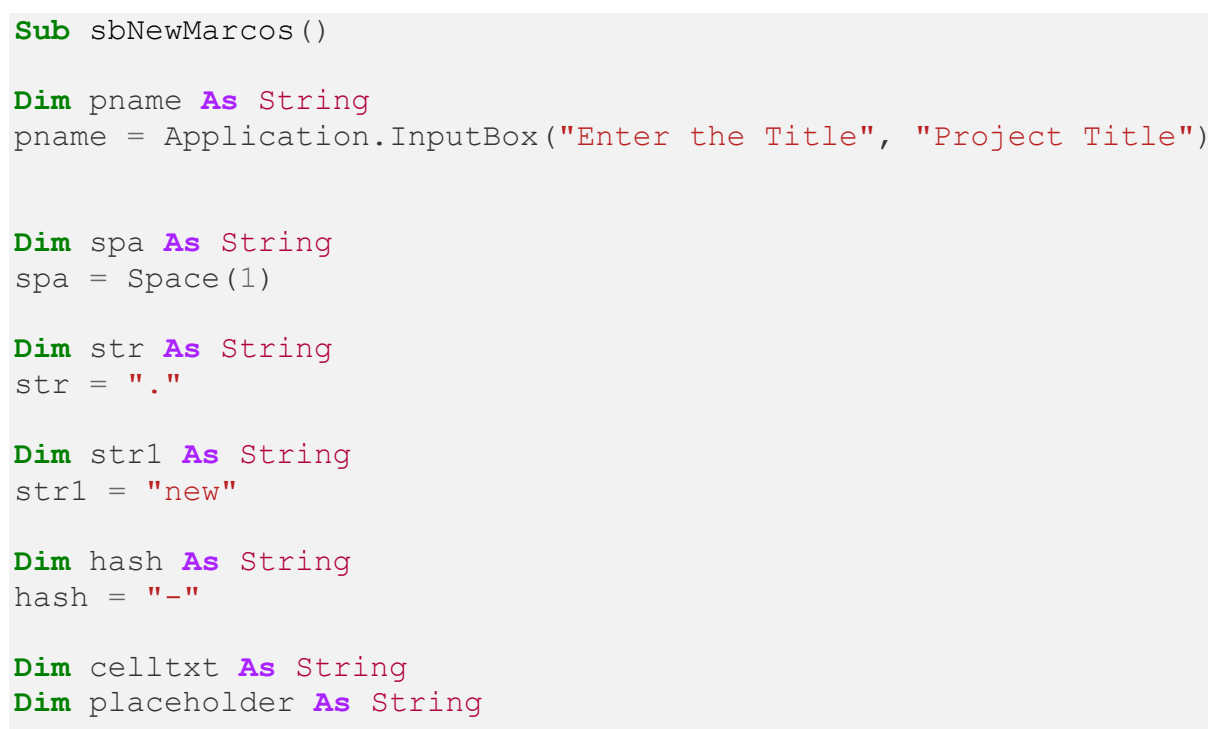




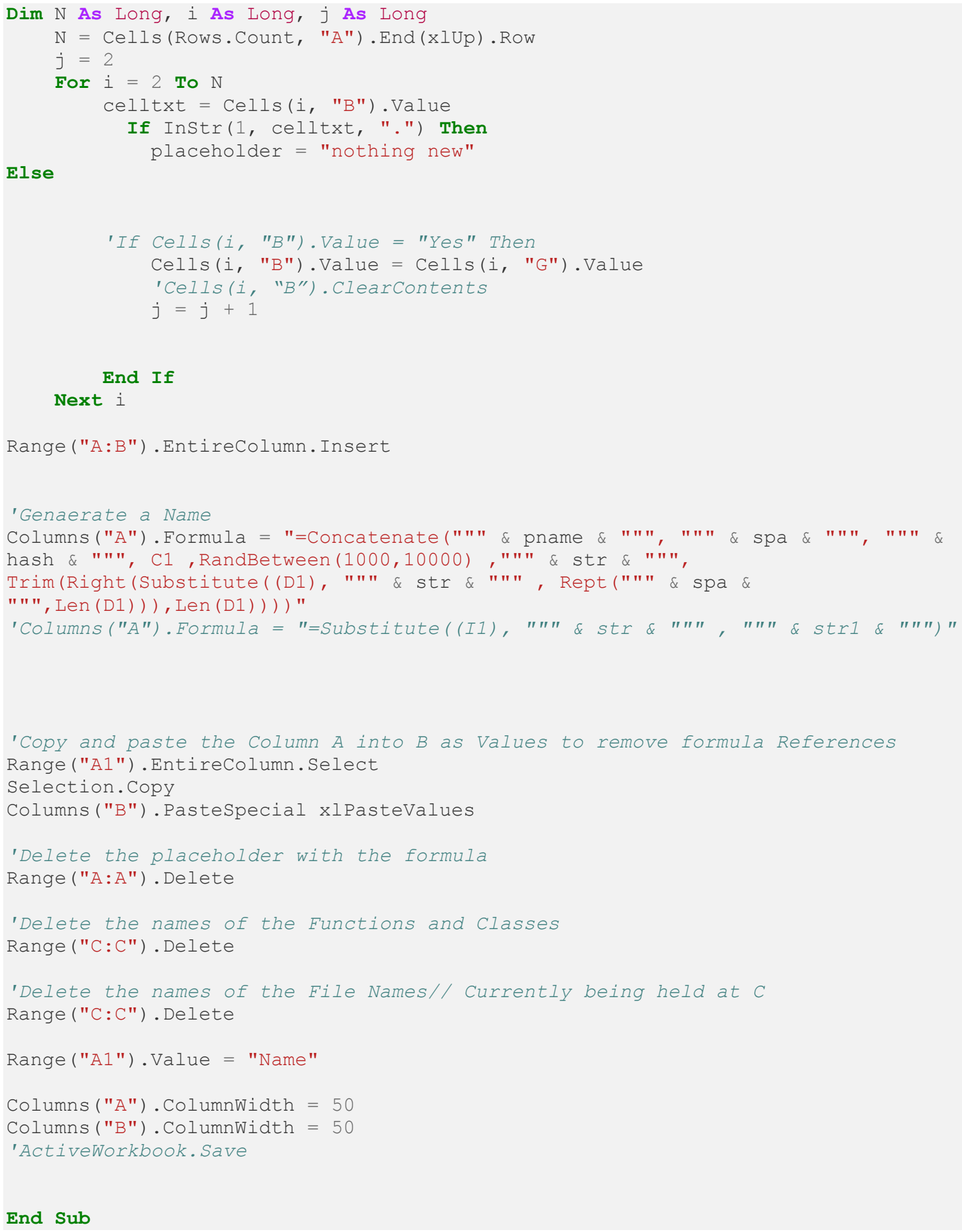

End Sub 